\title{
Flying Qualities and Controllability of Hypersonic Spaceplanes
}

Master of Science Thesis

Giulia Viavattene

항 



\title{
Flying Qualities and Controllability of Hypersonic Spaceplanes
}

\author{
Master of Science Thesis
}

by

\section{Giulia Viavattene}

in partial fulfillment of the requirements for the degree of

\author{
Master of Science \\ in Aerospace Engineering
}

at Delft University of Technology

to be defended publicly on Wednesday March 28, 2018 at 14:15

\begin{abstract}
Student number: $\quad 4519256$
Supervisor: $\quad$ Dr. ir. Erwin Mooij, TUDelft

Committee members: $\quad$ Prof. Dr. Ir. P.N.A.M. Visser, TUDelft

Dr. Ir. C. C. de Visser, TUDelft

Dr. Ir. E. Mooij, TUDelft

Cover Picture: $\quad$ Sänger II after the successful separation phase between the first and second stage. It was offered to Eugen Sänger by the industrial graphic artist Theo Lässig, 1964.
\end{abstract}




\section{Acknowledgments}

My experience at TU Delft started in September 2015. From that moment, everyday I have had the occasion to learn something new. Every lesson was so enlightening and I had the pleasure to share tip, ideas and experience with many of the lectures and colleagues. For this and many other reasons, I would like to thank them. Also, I would like to thank all the $9^{\text {th }}$ floor and, especially, the my master room mates for the positive working environment.

During my MSc thesis project, I have received more than valuable support from my supervisor, Erwin Mooij. I would like to thank Erwin for providing me all the concepts and suggestions that I needed to conduct this work and, particularly, to train me to "t(h)rust" myself.

My greatest thought goes to my family, my solid certainty. I would like to thank my parents, Lucia and Antonio, and my brother, Stefano. Thank you for believing in me and sustaining me in all my decisions, thank you for having made this dream possible, thank you for all the hugs that you gave me despite the 2,000 km of distance.

I would like to thank my grandparents, Silvia, Tanina, Angelo e Angelino, and relatives, Marco, Roxana, Zia Lolli, Zia Rita, Zio Antonio, Zio Salvo. Thank you for your constant and kind love. In particular, I would like to thank my grandmother, Nonna Tanina, because her prayers can reach and protect me wherever I am. Also, a special thanks goes to Zia Rita: she was my first teacher and she contributed to my attitude to the study and learning.

In addition, I am very grateful for the affection I received from my friends during the years I spent in Delft. Thank you for letting me feel like home in Delft.

Mostly, I would like to thank Federico, for continuously encouraging me and for making my every day the best day ever. 
La forza dell'ottimismo.

The power of optimism.

Ai miei genitori 


\section{Summary}

Spaceplanes represent a new promising concept for space flight. What make the spaceplanes so attractive are the possibility of reusing the system for more than one mission, and the flexibility that they allow in mission. It is expected that the spaceplane-mission demand will grow in the coming years, requiring that these vehicles have adequate properties of safety of flight and ease of controllability in nominal and off-nominal conditions. Thus, the flying qualities and controllability analysis of hypersonic spaceplanes, together with the development of an attitude controller capable of improving these properties, poses a challenging problem.

The main problem definition or research question of this thesis study has been formulated as follows:

Which are the flying qualities of hypersonic spaceplanes, how do the environmental disturbances and design uncertainties influence them, and how can a control system compensate for any deficiency of the system and contribute to the mission success?

After the investigation of different spaceplane concepts, the Festip System Study concept 1 (FSS-1) is selected as reference vehicle. The design points, where the analyses of this thesis work will be performed, are defined from the FSS- 1 ascent and re-entry trajectory. From the reference vehicle and mission, the mission and system requirements are defined. To simulate the motion of a spaceplane subjected to forces and moments of gravitation, aerodynamic and propulsive origin, and to compute its performance, a flight simulator is required. The Generic Attitude and Orbit Control Simulator (GAOCS) is used. Also, to study the spaceplane stability properties, the equations of motion need to be linearised and expressed in the state-space model.

The eigenmotion of the vehicle in open-loop can be identified by investigating the eigenvalues and eigenvectors of the state matrix of the state-space model. The trim capabilities of the system are studied to determine whether they improve stability. After having identified the flying-quality requirements from the military documents MIL-F-8785C and MIL-HDBK-1797 for subsonic aircraft, the flying qualities of the FSS-1 hypersonic vehicle are computed and compared with these requirements. These stability analyses converge to a unique, shared conclusion: the FSS-1 reference vehicle is dynamically unstable and it does not met the flying-quality requirements. So, it requires a control system to guarantee safety of flight and realise the mission objectives.

The development of a control system represents one of the main goal of the current study. Two concepts have been studied: the Linear-Quadratic-Regulator (LQR) and the Simple Adaptive Control (SAC). An optimal control-system design is identified by maximising the performance metrics of the integrated control error and effort. However, it is desirable that the control system is also as insensitive to disturbances as possible. To this end, a double-loop sensitivity analysis process, known as robust design methodology, is set up. So, a robust control-system is designed.

To examine the controllability properties of the FSS-1 spaceplane, the military requirements are used. The response to angle-of-attack and bank-angle commands in nominal conditions and the response to atmospheric disturbances are simulated and evaluated. The analyses show that both LQR and SAC can realise the attitude commands. However, when perturbations are introduced, the LQR response starts drifting away, requiring large control-surface deflections that lead to diverging oscillations and a complete loss of control. This does not happen when the SAC controller is used, since it can adapt its activity to counteract the disturbances thanks to its adaptive gains. Also, it is demonstrated the optimal control design cannot handle uncertainties in the input parameters, since it leads to saturation of the control variables. Differently, the robust design minimises the influence of uncertainties over the system performance: the control commands can be executed with an acceptable control effort. Thus, the SAC robust control system is able to stabilise and control the vehicle so that the safety of flight and capability to achieve the mission success are guaranteed.

In conclusion, a spaceplane is characterised by poor flying qualities and stability characteristics along its hypersonic ascent and re-entry trajectory. However, when a robust and advanced control system is integrated into the system, it becomes stable and controllable, allowing for a safe flight and the mission success. Also, the system behaviour is related to the control system performance. It is fundamental that the control system is designed to meet the mission requirements, focusing on the compromise between optimality and robustness. 


\section{Contents}

Acknowledgments iii

Summary vii

List of Symbols

List of Abbreviations $\quad$ XV

1 Introduction 1

1.1 Mission Heritage . . . . . . . . . . . . . . . . . . . . . . . . . . . . . . . 1

1.1.1 Flying Qualities: from Subsonic to Hypersonic Vehicles . . . . . . . . . . . . . . . . 2

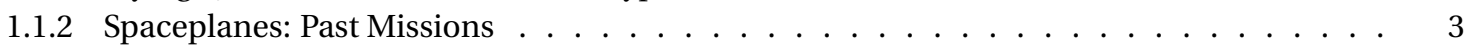

1.2 Research Question . . . . . . . . . . . . . . . . . . . . . . . . 4 4

2 Mission \& System Definition 7

2.1 Reference Vehicle . . . . . . . . . . . . . . . . . . . . . . . 7

2.1.1 Aerodynamic System. . . . . . . . . . . . . . . . . . . . . . . . . 9

2.1 .2 Aero-data Verification . . . . . . . . . . . . . . . . . . . . 13

2.1.3 Propulsion System . . . . . . . . . . . . . . . . . . . . . . . . . 14

2.2 Reference Mission . . . . . . . . . . . . . . . . . . . . . . . . . 16

2.3 Mission and System Requirements . . . . . . . . . . . . . . . . . . . . . . . . . 17

3 Environment 19

3.1 Earth's Shape and Gravitational Field . . . . . . . . . . . . . . . . . . . . . . . . . . . 19

3.2 Atmosphere. . . . . . . . . . . . . . . . . . . . . . . . 20

3.3 Wind Model. . . . . . . . . . . . . . . . . . . . . . . . . . . . . . 21

3.3.1 Steady-State Wind . . . . . . . . . . . . . . . . . . . . . . . 21

3.3.2 Wind Gusts. . . . . . . . . . . . . . . . . . . . . . . . . . . . . . . . . . . . . . . . . . . . . . . . . . .

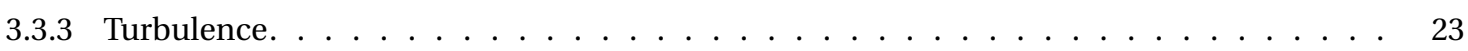

4 Flight Mechanics $\quad 25$

4.1 Dynamic Models Blueprint . . . . . . . . . . . . . . . . . . . . . . . . . . 25

4.2 Reference Frames . . . . . . . . . . . . . . . . . . . . . . . . 27

4.3 State Variables . . . . . . . . . . . . . . . . . . . . . . . . . . . . 29

4.3.1 Position and Velocity. . . . . . . . . . . . . . . . . . . . . . . . . . . . . . . . . . . . . . . .

4.3 .2 Attitude and Angular Rate . . . . . . . . . . . . . . . . . . . . . . . . . . . . . . . . . . . . . . .

4.4 Frame Transformations . . . . . . . . . . . . . . . . . . . . . . . . . . 32

4.5 Force Models . . . . . . . . . . . . . . . . . . . . . . . . . . . . . . 34



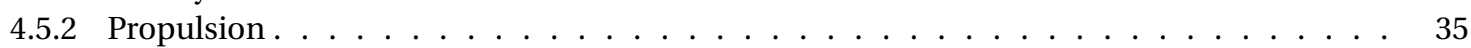

4.6 Equations of Motion: General Formulation . . . . . . . . . . . . . . . . . . . . . . 37

4.6.1 Equations of the Translational Motion . . . . . . . . . . . . . . . . . . 37

4.6.2 Equations of the Rotational Motion . . . . . . . . . . . . . . . . . . . 39

4.6 .3 Wind Equations . . . . . . . . . . . . . . . . . . . . . . . . 41

4.7 State-Space Form . . . . . . . . . . . . . . . . . . . . . . . . . . . . 42



4.7.2 Closed-Loop Rotational Model. . . . . . . . . . . . . . . . . . . . . 43

5 Stability \& Flying Qualities $\quad 45$

5.1 Eigenmotion . . . . . . . . . . . . . . . . . . . . . . . . 4 45

5.1.1 Stability Problems in Hypersonic Flight . . . . . . . . . . . . . . . . . . . . . . . 48

5.2 Military Specifications . . . . . . . . . . . . . . . . . . . . . . 50

5.2 .1 Longitudinal Requirements . . . . . . . . . . . . . . . . . . . 51

5.2 Lateral-Directional Requirements . . . . . . . . . . . . . . . . . . . . . 51 51

5.2 .3 Atmospheric Disturbances. . . . . . . . . . . . . . . . . . . . . 52 
5.3 Quality Assurance. . . . . . . . . . . . . . . . . . . . . . . . . . . . . 52

5.4 Stability \& Flying-Quality Analyses . . . . . . . . . . . . . . . . . . . . . . . . . . 53

5.4 Eigenmotion of Hypersonic Vehicles. . . . . . . . . . . . . . . . . . . . . 53

5.4 .2 Trim Analysis . . . . . . . . . . . . . . . . . . . . . . . . . . 56

5.4 .3 Flying Qualities . . . . . . . . . . . . . . . . . . . . . . 57

6 Control-System Design $\quad 61$

6.1 Feedback Control . . . . . . . . . . . . . . . . . . . . . . . . . . . . . . 62

6.1 Performance Indices . . . . . . . . . . . . . . . . . . . . . . 63

6.2 Optimal Control Theory. . . . . . . . . . . . . . . . . . . . . . . . . . 65

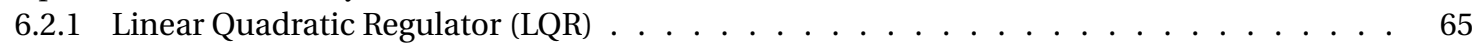

6.2 LQR for Reference Vehicle . . . . . . . . . . . . . . . . . . . . . . . . . . . . . . . . . . . . . . . . . . 65

6.3 Model Reference Adaptive Control . . . . . . . . . . . . . . . . . . . . . . . . . . 69

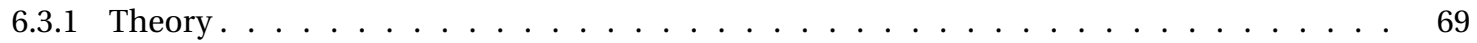

6.3 .2 Reference Model . . . . . . . . . . . . . . . . . . . . . . . 71

6.3.3 Baseline Controller Design. . . . . . . . . . . . . . . . . . . . . . . . . 72

6.4 Actuator Assignment . . . . . . . . . . . . . . . . . . . . . . . . . . 76

7 Robust Design \& Controllability 79

7.1 Robust Design Methodology ． . . . . . . . . . . . . . . . . . . . . . . . . . . . . . . . . . 79

7.2 Robust Control-System Design . . . . . . . . . . . . . . . . . . . . . . . 80

7.2.1 Longitudinal Motion . . . . . . . . . . . . . . . . . . . . . . 81

7.2 Lateral Motion . . . . . . . . . . . . . . . . . . . . . . . . 81

7.3 Controllability . . . . . . . . . . . . . . . . . . . . . . . 83

7.4 Response to Atmospheric Disturbances. . . . . . . . . . . . . . . . . . . . . . . 85

7.4.1 Response to Steady-State Wind . . . . . . . . . . . . . . . . . . . . . . . . 86

7.4 .2 Response to Wind Gusts . . . . . . . . . . . . . . . . . . . . . . . . . . . . 87

7.4 .3 Response to Turbulence . . . . . . . . . . . . . . . . . . . . . . 88

8 Conclusions \& Recommendations $\quad 91$

8.1 Conclusions. . . . . . . . . . . . . . . . . . . . . . . . 91

8.2 Recommendations ............................. 93

Bibliography 95

A State-Space Matrix Coefficients $\quad 99$

A.1 Open-loop Model . . . . . . . . . . . . . . . . . . . . . . . . . . . . 100

A.2 Closed-Loop Rotational Model . . . . . . . . . . . . . . . . . . . . . . . . . . . . . . . 102

B Software Architecture \& Verification $\quad 105$

B.1 Software Architecture . . . . . . . . . . . . . . . . . . . . . . . . . 105

B.2 Verification ............................... 108

B.2.1 Linear System \& Eigenmotion Evaluation . . . . . . . . . . . . . . . . . . . 108

B.2.2 Control System. . . . . . . . . . . . . . . . . . . . . . . . . . . . 109 


\section{List of Symbols}

\section{Roman Letters}

\begin{tabular}{|c|c|c|}
\hline $\mathrm{a}$ & Speed of Sound & {$[\mathrm{m} / \mathrm{s}]$} \\
\hline $\mathrm{a}_{i j}$ & Elements of State Matrix A & [varies] \\
\hline a & Acceleration Vector & {$\left[\mathrm{m} / \mathrm{s}^{2}\right]$} \\
\hline $\mathrm{A}_{e}$ & Exit Area & {$\left[\mathrm{m}^{2}\right]$} \\
\hline $\mathbf{A}$ & State or System Matrix & [varies] \\
\hline B & Control Input Matrix & [varies] \\
\hline $\mathrm{b}_{i j}$ & Elements of Control Matrix B & [varies] \\
\hline $\mathrm{b}_{\text {ref }}$ & Aerodynamic Reference Length & {$[\mathrm{m}]$} \\
\hline$C_{A B}$ & Transformation Matrix from frame A to frame B & {$[-]$} \\
\hline $\mathbf{C}$ & Output Matrix & [varies] \\
\hline $\mathrm{C}_{D}$ & Drag Force Coefficient & {$[-]$} \\
\hline $\mathrm{C}_{l}$ & Roll Moment Coefficient & {$[-]$} \\
\hline $\mathrm{C}_{L}$ & Lift Force Coefficient & {$[-]$} \\
\hline $\mathrm{C}_{m}$ & Pitch Moment Coefficient & {$[-]$} \\
\hline $\mathrm{C}_{n}$ & Yaw Moment Coefficient & {$[-]$} \\
\hline $\mathrm{c}_{\text {ref }}$ & Aerodynamic Reference Length & {$[\mathrm{m}]$} \\
\hline $\mathrm{C}_{S}$ & Side Force Coefficient & {$[-]$} \\
\hline $\mathrm{d}$ & Distance & {$[\mathrm{m}]$} \\
\hline $\mathrm{d}_{x, y, z}$ & Distance Centre of Thrust/Centre of Mass (X,Y,Z-axis) & {$[\mathrm{m}]$} \\
\hline $\mathrm{D}$ & Drag Force & {$[\mathrm{N}]$} \\
\hline $\mathrm{e}$ & Error & [varies] \\
\hline $\mathrm{f}$ & Function & [varies] \\
\hline $\mathbf{F}$ & Force Vector & {$[\mathrm{N}]$} \\
\hline $\mathscr{F}$ & Reference Frame & {$[-]$} \\
\hline $\mathbf{g}$ & Gravitational Acceleration Vector & {$\left[\mathrm{m} / \mathrm{s}^{2}\right]$} \\
\hline $\mathrm{g}$ & Gravitational Acceleration & {$\left[\mathrm{m} / \mathrm{s}^{2}\right]$} \\
\hline h & altitude & {$[\mathrm{m}]$} \\
\hline $\mathrm{H}_{s}$ & Scale Height & {$[\mathrm{m}]$} \\
\hline I & Identity Matrix & {$[-]$} \\
\hline I & Inertia Tensor & {$\left[\mathrm{kgm}^{2}\right]$} \\
\hline I & Moment/Product of Inertia & {$\left[\mathrm{kgm}^{2}\right]$} \\
\hline $\mathrm{I}_{s p}$ & Specific Impulse & [s] \\
\hline $\mathrm{J}$ & Cost Criterion & [varies] \\
\hline K & Control Gain & {$[-]$} \\
\hline $\mathbf{K}$ & Control Gain Matrix & {$[-]$} \\
\hline $\mathrm{L}$ & Lift Force & {$[\mathrm{N}]$} \\
\hline $\mathrm{L}_{r e f}$ & Aerodynamic Reference Length & {$[\mathrm{m}]$} \\
\hline $\mathscr{L}$ & Roll Moment & {$[\mathrm{Nm}]$} \\
\hline $\mathrm{m}$ & Mass & {$[\mathrm{kg}]$} \\
\hline$\dot{m}$ & Mass Flow & {$[\mathrm{kg} / \mathrm{s}]$} \\
\hline M & Mach Number & {$[-]$} \\
\hline $\mathscr{M}$ & Pitch Moment & {$[\mathrm{Nm}]$} \\
\hline$M_{T_{x, y, z}}$ & Propulsion Moments (X,Y,Z-axis) & {$[\mathrm{Nm}]$} \\
\hline $\mathbf{M}$ & Moment Vector & {$[\mathrm{Nm}]$} \\
\hline $\mathrm{n}$ & Load Factor & {$[-]$} \\
\hline $\mathscr{N}$ & Yaw Moment & {$[\mathrm{Nm}]$} \\
\hline $\mathrm{N}_{A}$ & Avogadro's Constant & {$\left[\mathrm{kmol}^{-1}\right]$} \\
\hline $\mathrm{p}$ & Roll Rate & {$[\mathrm{rad} / \mathrm{s}]$} \\
\hline $\mathrm{p}$ & Pressure & {$[\mathrm{Pa}]$} \\
\hline
\end{tabular}


$\mathrm{R}_{e}$

$\mathbf{T}_{i}$

$\mathbf{T}_{p}$

X, Y, Z

$\mathrm{y}$

$\mathrm{y}$

y

Solution of Matrix Riccati Equation

$[-]$

Period

Atmospheric Pressure

Pitch Rate

Quaternion

Dynamic Pressure

State Deviation Weight Matrix

Position Vector

Yaw Rate

Modulus of Position Vector

Air Gas Constant

Control Effort Weight Matrix

Equatorial Radius

Laplace Variable

Side Force

Aerodynamic Reference Surface

Time

Rise Time

Weighting Matrix Integral Gain

Weighting Matrix Proportional Gain

Modulus of Thrust Vector

Thrust Vector

Temperature

Time-to-Halve the Amplitude

Time-to-Double the Amplitude

Control Vector

Wind Velocity Component

Wind Velocity Component

Modulus of the velocity vector

Velocity vector

Wind Velocity vector

Cartesian position components

State Vector

Axes

Cross Range

Response

Output Vector

[s]

$\left[\mathrm{N} / \mathrm{m}^{2}\right]$

$[\mathrm{rad} / \mathrm{s}]$

[-]

[Pa]

$[-]$

[m]

$[\mathrm{rad} / \mathrm{s}]$

[m]

$[\mathrm{J} / \mathrm{kg} \mathrm{K}]$

[-]

[m]

[-]

[n]

$\left[\mathrm{m}^{2}\right]$

[s]

[s]

[-]

[-]

[N]

$[\mathrm{N}]$

$[\mathrm{K}]$

[s]

[s]

[-]

$[\mathrm{m} / \mathrm{s}]$

$[\mathrm{m} / \mathrm{s}]$

$[\mathrm{m} / \mathrm{s}]$

$[\mathrm{m} / \mathrm{s}]$

$[\mathrm{m} / \mathrm{s}]$

[m]

[-]

$[-]$

[m]

$[-]$

$[-]$

\section{Greek Letters}

$\alpha$
$\beta$
$\gamma$
$\Delta$
$\delta$
$\delta_{a}$
$\delta_{b}$
$\delta_{e}$
$\delta_{r}$
$\delta_{T}$
$\delta_{w}$
$\epsilon$
$\epsilon_{T}$
$\eta$
$\eta_{x, y, z}$
$\zeta$
$\theta$

Angle of attack

[rad]

Sideslip Angle

[rad]

Flight-path angle

[rad]

Deviation

Geocentric Latitude

[-]

Aileron Deflection Angle

[rad]

Body-flap Deflection Angle

[rad]

[rad]

Elevator Deflection Angle

[rad]

Rudder Deflection Angle

[rad]

Throttle Setting

[-]

Wing-flap Deflection Angle

[rad]

Perturbation parameter

[-]

Thrust Elevation Angle

[rad]

Signal-to-Noise Ratio

$[\mathrm{dB}]$

Commanded Moment Fraction

[-]

Damping Ratio

[-]

Pitch angle 
Gravitation Parameter

$\left[\mathrm{m}^{3} / \mathrm{s}^{2}\right]$

Density

Bank angle

$\left[\mathrm{kg} / \mathrm{m}^{3}\right]$

[rad]

Standard Deviation

[-]

Geocentric Longitude

[rad]

Time Constant

[s]

Heading Angle

[rad]

Yaw Angle

[rad]

Thrust Azimuth Angle

[rad]

Natural Frequency of Eigenmotion

$[\mathrm{rad} / \mathrm{s}]$

Earth's rotational rate

$[\mathrm{rad} / \mathrm{s}]$

$\omega$ Rotation Vector

$[\mathrm{rad} / \mathrm{s}]$ 


\section{List of Abbreviations}

\begin{tabular}{|c|c|}
\hline $\mathrm{CDF}$ & Cumulative Density Function \\
\hline CoM & Centre of Mass \\
\hline CoT & Centre of Thrust \\
\hline CPU & Central Processing Unit \\
\hline DoF & Degrees of Freedom \\
\hline EoM & Equations of Motion \\
\hline FESTIP & Future European Space Transportation Investigations Program \\
\hline FSS-1 & Festip System Study concept 1 \\
\hline GAOCS & Generic Attitude and Orbit Control \\
\hline GNC & Guidance, Navigation and Control \\
\hline HWM07 & Horizontal Wind Model 2007 \\
\hline LQR & Linear Quadratic Regulator \\
\hline LTI & Linear Time-Invariant \\
\hline MECO & Main Engines Cut-Off \\
\hline MIMO & Multiple Input Multiple Output \\
\hline MRAC & Model Reference Adaptive Control \\
\hline NASA & National Aeronautics and Space Administration \\
\hline ODE & Ordinary Differential Equation \\
\hline $\mathrm{O} / \mathrm{F}$ & Oxidizer/Fuel Mixture Ratio \\
\hline PDF & Probability Density Function \\
\hline PID & Proportional-Integrative-Derivative \\
\hline PSD & Power Spectral Densities \\
\hline RCS & Reaction Control Thrusters \\
\hline RK4 & Fourth-Order Runge-Kutta method \\
\hline RNG & Random Number Generator \\
\hline SAC & Simple Adaptive Control \\
\hline SISO & Single Input Single Output \\
\hline SNR & Signal-to-Noise Ratio \\
\hline SSTO & Single Stage To Orbit \\
\hline TSTO & Two Stage To Orbit \\
\hline TUDAT & TU Delft Astrodynamics Toolbox \\
\hline TVC & Thrust Vectoring Control \\
\hline US76 & United States Standard Atmosphere 1976 \\
\hline US & United States \\
\hline VTO-HL & Vertical Take-off, Horizontal Landing \\
\hline WCC & Winged-Cone Configuration \\
\hline \multicolumn{2}{|c|}{ Reference Frames $\mathscr{F}$} \\
\hline AA & Airspeed-Based Aerodynamic Frame \\
\hline AG & Groundspeed-Based Aerodynamic Frame \\
\hline B & Body Frame \\
\hline I & Inertial Earth-Centred Frame \\
\hline $\mathrm{P}$ & Propulsion Frame \\
\hline $\mathrm{R}$ & Rotating Earth-Centred Frame \\
\hline TA & Airspeed-Based Trajectory Frame \\
\hline TG & Groundspeed-Based Trajectory Frame \\
\hline $\mathrm{V}$ & Vertical Frame \\
\hline $\mathrm{W}$ & Wind Frame \\
\hline
\end{tabular}




\section{1}

\section{Introduction}

Spaceplanes constitute the new frontier for space flight. The concept was born from the idea of combining some of the features of aircraft and spacecraft to create an advanced vehicle. It can generate lift during its atmospheric flight, analogously to an aircraft, and it is able to travel in space as a spacecraft. For this reason it was called spaceplane. A spaceplane is a reusable, safe, efficient and economical space transportation system. In addition to reusability, the main characteristic that makes it so attractive for future space missions is the flexibility that it allows in mission: for instance, it can be launched from any site and perform a cruise flight. It follows that multiple missions can be performed with the same system. This allows to reduce the launch costs and the turn-around time with respect to conventional rocket launchers [Bentley, 2009].

The current rocket market, actually based on satellite launches and manned missions, is expected to change considerably in the coming years. Indeed, although the market for expensive systems is difficult to foresee, the prediction is that space tourism and hypersonic passenger transport will finance the development of spaceplanes. This expected growing demand of spaceplane missions requires these systems to be characterised by satisfactory capabilities in terms, in particular, of safety of flight and ease of controlling in steady-state flight, during manoeuvres and even in presence of unforeseen perturbations. It is the goal of this thesis project to contribute to this research field, analysing the flying qualities and controllability characteristics of hypersonic spaceplanes.

Flying qualities, also known as handling qualities, involve the study and evaluation of the stability and control characteristics of an aircraft. They have the purpose of ensuring the safety of flight and the ease of controlling the vehicle in steady flight and during manoeuvres. Thus, the compliance of the vehicles with the flying qualities and controllability characteristics is necessary to adequately perform the mission objectives and operate within the safety boundaries. Flying-quality requirements change depending on the type of vehicle and flight phase category. Usually, they involve [Mooij, 1985]:

- the efficacy of a control to generate the whole range of manoeuvres or steady equilibrium states, starting from a performance standpoint;

- the limits of the control forces to induce state changes;

- the manoeuvrability from one steady-state condition to another;

- the capability of maintaining an equilibrium condition with constant speed and angle of attack if the controls are not operating;

- the speed of the system response to a command.

Section 1.1 consists of a study of the spacaplanes and flying qualities in the context of their mission heritage. It is followed by the definition of the main research questions in Section 1.2. Finally, the methodology and structure of the report are depicted.

\subsection{Mission Heritage}

Usually, a spaceplane has the shape of a spacecraft equipped with wings that are useful while flying in the atmosphere and a propulsion system that allows it to reach space. Many concepts of reusable launch systems have been studied. The main differences between them concern the number of stages, the type of propulsion 


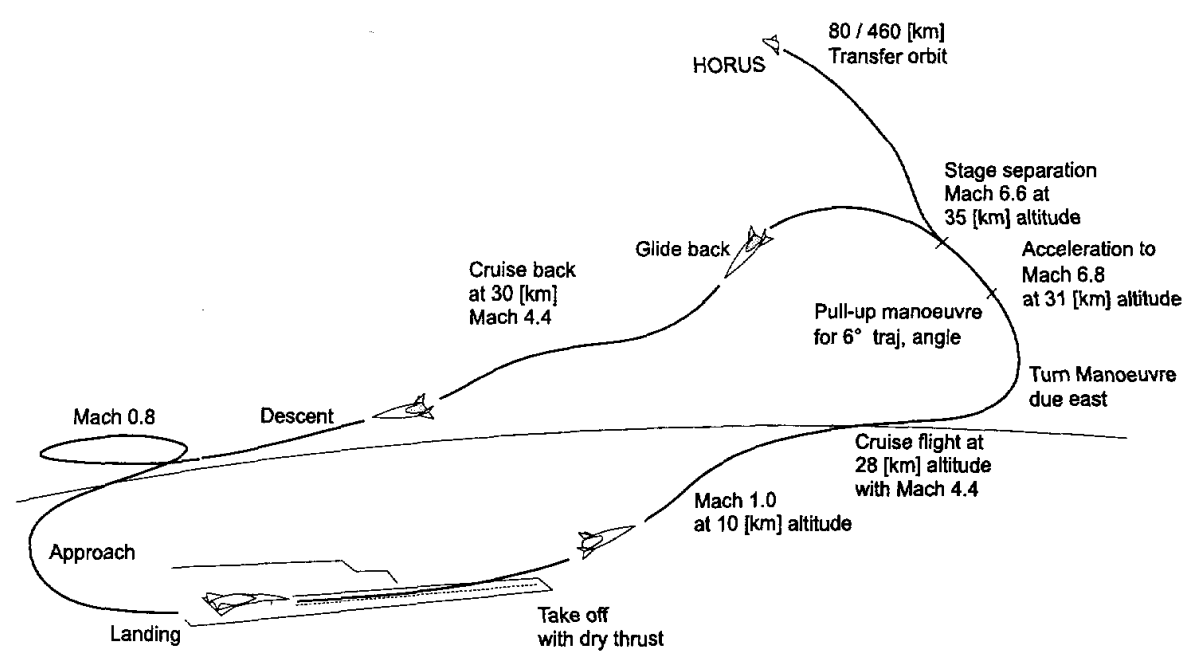

Figure 1.1: Sänger II typical mission with its second stage HORUS [Koelle and Kuczera, 1989a].

system, and the method to take-off and landing. A spaceplane is defined to be single-stage-to-orbit (SSTO), when it presents one stage, and two-stage-to-orbit (TSTO), when it features two stages. The propulsion system could be rocket based or constituted by airbreathing jet engines. Also, depending on the mission definition, a spaceplane can take off and land horizontally or vertically [Mehta and Bowles, 2001].

An SSTO spaceplane is a single vehicle that can perform an orbital or sub-orbital flight. The orbital flight is theoretically possible but at the expense of a very small payload capability. The take-off is generally vertical and, once it has climbed to orbit, the orbit is acquired and the payload is deployed. Finally, after it has completed its mission, it performs the re-entry flight and lands horizontally or vertically. A TSTO spaceplane comprises a first stage, known as carrier, and a conventional rocket second stage, known as orbiter. Usually, the propulsion of the first stage is airbreathing because it allows for a greater overall performance and operability than rocket propulsion. Indeed, using air as oxidiser reduces the weight and the cost because of a better propulsion efficiency and thermal cooling capability. In this case, both the take-off and landing are horizontal. The typical mission phases of a TSTO spaceplane are illustrated in Figure 1.1 [Mehta and Bowles, 2001].

Spaceplanes have been studied since the early twentieth century, and several types of vehicles have been proposed. Currently, five spaceplanes have flown successfully. These are the North American X-15, Space Shuttle, Buran, SpaceShipOne, and Boeing X-37. After they have entered space, they re-entered the Earth's atmosphere and safely landed on Earth. Space Shuttle, Buran and Boeing X-37 were characterized by a vertical ascent phase with a rocket-based propulsion and horizontal landing. The North American X-15 and SpaceShipOne were air-launched and equipped with rocket engines. In this case, the wings were used to create lift not only in the re-entry phase, but also during take-off. Moreover, Space Shuttle, Buran and Boeing X-37 entered an orbit around Earth, while North American X-15 and SpaceShipOne performed a sub-orbital flight. Also, Buran and X-37 have flown only unmanned missions, differently from Space Shuttle, X-15 and SpaceShipOne, that have been piloted [Bentley, 2009].

Past and recent missions and studies about flying qualities, controllability, and spaceplanes are presented in the following of this section to provide some background information to justify the need for the thesis mission. Section 1.1.1 introduces the evolution of flying qualities over the course of time, with a short presentation of the main documents that constitute the mission heritage for this thesis project. A survey on past space missions where spaceplanes have been employed is given in Section 1.1.2.

\subsubsection{Flying Qualities: from Subsonic to Hypersonic Vehicles}

The first analysis of flying qualities dates back to the first manned flight with a motorised airplane. Understanding how to balance, steer, and control the airplane appeared to be of extreme importance from the very first moment. After the end of World War II, the first standard document defining the flying-quality requirements and agreed to by all the military services was published in response to the need to make aircraft operations and responses to pilot control uniform. This document was updated in 1980, given the advances in aircraft technology, to improve safety in any flight phase and allow the pilot to perform mission tasks with 
precision, smoothness, and minimum effort. MIL-F-8785C [1980] was issued to define the standards about the aircraft dynamic response [Cotting, 2010].

Later, the MIL-F-8785C specifications were extended for digitally controlled aircraft and merged with flighttest specifications into one single document, MIL-HDBK-1797 [1997]. These two MIL-Specs documents define flying-quality requirements for all the categories of aircraft flying in the subsonic regime. However, the study conducted by Cox and Jackson [1997] shows that one category presents characteristics that resemble those of supersonic and hypersonic vehicles. The aircraft with these characteristics belong to Group III of large, heavy, low-to-medium manoeuvrability aircraft. Although the control system of hypersonic aircraft shall be designed to meet the requirements specified in the MIL standards, the differences between subsonic and high-speed regimes should be taken into account. The main differences are a higher kinetic energy level, which causes a change in the interrelation between height and speed excursions during a perturbation, and an additional longitudinal mode of motion, the height mode. Finally, it is important to notice that long-term dynamic characteristics influence control and stability in a different way depending on the flight regime.

The flying qualities requirements are determined using stability and control parameters. For each of these parameters, the boundary values that define the level of flying qualities are specified. The levels of flying qualities are acceptability levels to perform the operational mission and they can be presented as follows [MILHDBK-1797, 1997]:

- Level 1: "Flying qualities clearly adequate for the mission flight phase";

- Level 2: "Flying qualities adequate to accomplish the mission flight phase, but some increase in pilot workload or degradation in mission effectiveness, or both, exists";

- Level 3: "Flying qualities such that the aircraft can be controlled safely, but pilot workload is excessive or mission effectiveness is inadequate, or both". However, Category B flight phases can be completed.

The noteworthy past analyses about flying qualities and controllability of hypersonic spaceplanes constitute part of the mission heritage. Chalk [1980] provides important results about flying qualities for supersonic flight, performed with Concorde, that could be used also to conduct the analysis of flying qualities in hypersonic conditions. These studies showed that some MIL requirements can be, in certain aspects, too restrictive for high-speed vehicles due to the much slower dynamics with respect to subsonic aircraft. For instance, the MIL criterion about time delay for which more relaxed criterion could be admitted for this aircraft category.

Jacob et al. [2005] and Cox et al. [1995] present the results of a simulation and flight test experiment programme for research about flying qualities for hypersonic vehicles, conducted by the Technische Universität München in cooperation with the NASA Dryden Flight Research Center. The latter provided test facilities for flying quality analysis, which are an SR-71 aircraft, an operational SR-71 simulator and a dedicated flight simulator for hypersonic vehicles. The SR-71 aircraft is a supersonic vehicle that can reach a speed higher than Mach 3 and an altitude greater than $21 \mathrm{~km}$. Considering the large similarities of the high-supersonic and the hypersonic flight regimes, SR-71 represents an extraordinary opportunity to study flying characteristics under hypersonic conditions. The operational SR-71 simulator was useful for the research conducted during the flight test preparation and when separate simulator experiments were needed. These test facilities present a control system designed to obtain Level 1 in the MIL-requirements.

However, these previous studies focused on particular flight conditions and/or vehicles with peculiar characteristics. Thus, the results cannot be generalised to define new flying qualities requirements for hypersonic spaceplanes. It follows that it is fundamental to expand the study of flying qualities and controllability characteristics of spaceplanes for a larger range of flight conditions.

\subsubsection{Spaceplanes: Past Missions}

To achieve the thesis objectives, a reference vehicle to conduct the simulation and the analysis needs to be selected. Given the considerable number of past and recent studies and missions, the attention will be focused on those whose mass and inertia, aerodynamics, and propulsion databases are fully or partially available. In particular, two SSTO spaceplanes, which are the Winged-Cone Configuration, FESTIP concept 1, and a TSTO spaceplane that is a vehicle proposed by GMV, are considered.

The winged-cone configuration (WCC) is a manned, horizontal take-off and landing, SSTO, airbreathing launch vehicle. On the basis of the information provided by Shaughnessy et al. [1990], WCC is defined as a generic SSTO configuration that is often used to examine ascent performance issues since the mass, aerodynamic, and propulsion models are suitable for point mass and batch and real-time 6 degrees-of-freedom 
(DoF) simulations. The vehicle is characterised by a gross take-off weight of around $140 \mathrm{t}$ and a fuselage length of about $60 \mathrm{~m}$. It is capable to control roll, pitch, and yaw motion by means of its control surfaces that are two elevons and one rudder. It has also two small canards, but they are deployed only in subsonic speed.

Festip System Study concept 1 (FSS-1) is a vehicle concepts of the Future European Space Transportation Investigations Programme (FESTIP) mission by Daimler-Benz Aerospace [1998]. The vehicle is a vertical takeoff, horizontal landing (VTO-HL) and SSTO with delta-shaped wings. This launch vehicle concept was derived from the HORUS configuration, which is the second stage of the TSTO launch vehicle Sänger II [Koelle and Kuczera, 1989b]. FSS-1 is propelled by 8 main rocket engines with a thrust level of $1800 \mathrm{kN}$. The ascent flight can be controlled by means of control surfaces, engine gimbal and throttle. The control surfaces are a body flap, two (left and right) elevons, and two (left and right) rudders. It has a gross take-off weight of $900 \mathrm{t}$ and a fuselage length of $59 \mathrm{~m}$. The mass model is defined assuming that the vehicle is a rigid structure and that, due to fuel expulsion for thrust generation, mass, the centre of mass (CoM) position and inertia moments and products change as function of fuel consumption. The aerodynamic model is complete, since it is possible to use the fully available database of HORUS. Also, the propulsion model is fully available: values of thrust level, chamber pressure, specific impulse, oxidizer/fuel $(\mathrm{O} / \mathrm{F})$ mixture ratio, nozzle expansion ratio. Moreover, adopting FSS1 as reference vehicle gives the possibility to use HORUS as benchmark for the software verification and for the analysis of the stability and flying qualities. Indeed, Brinkman [2017], Rijnsdorp [2017], and Mooij [1998a] study the attitude control of HORUS along its re-entry flight and Mooij [2013] provides an extensive study of the characteristic motion of re-entry vehicles, using HORUS as reference vehicle.

Finally, the last vehicle considered as potential reference vehicle to conduct this thesis analysis is the one proposed by GMV. One of its projects, documented by Carmona Castillo and Sanchez Gonzales Cantalejo [1993], involves the definition of a preliminary spaceplane's design to contribute to flight-dynamics simulation and GNC problems for hypersonic spaceplanes. It is a TSTO launch vehicle derived from the Sänger II configuration. The gross take-off weight is around $350 \mathrm{t}$ and the fuselage length is about $82 \mathrm{~m}$. Before the separation between first and second stages, the vehicle is propelled through the propulsion system of the first stage only, which is an airbreathing propulsion system. After separation, the rocket propulsion of the second stage is used. The vehicle can be controlled along its ascent trajectory through the aerodynamic control surfaces and propulsion control devices, i.e., power setting and thrust vectoring. The control surfaces are only two elevons for the first stage, which are used before separation, while the second stage has a body flap, two elevons and winglets. The mass-property and propulsion models are available. For what concerns the aerodynamic model, it is not fully available since only the 3-DoF longitudinal aerodynamic characteristics are specified. On the basis of these past missions, the reference vehicle for this thesis project will be selected in Section 2.1.

\subsection{Research Question}

Spaceplanes are generally unstable vehicles, thus to guarantee the mission success, they need to be provided with a Guidance, Navigation, and Control (GNC) system. Moreover, the stability and controllability properties of these vehicles change dramatically during the mission given the wide range of altitude and velocity experienced. It is for this reason that the GNC shall be designed so that the safety of flight can be ensured and the mission success can be achieved in nominal and off-nominal conditions.

The main research question that this thesis work desires to answer has been formulated as follows:

Which are the flying qualities of hypersonic spaceplanes, how do the environmental disturbances and design uncertainties influence them, and how can a control system compensate for any deficiency of the system and contribute to the mission success?

The main research question holds several research opportunities that can be expressed by the following research sub-questions:

1. Which are the flying qualities of hypersonic spaceplanes?

2. How do the flying qualities of hypersonic spaceplanes change with respect to the flying qualities of subsonic vehicles embodied in MIL-F-8785C [1980] and MIL-HDBK-1797 [1997]?

3. What are the controllability characteristics of hypersonic spaceplanes?

4. What is the influence of environmental disturbances, such as steady-state wind, turbulence and wind gusts, on the system performance?

5. If environmental disturbances introduce some deficiency in the system, how can the control system compensate for it? 
6. How sensitive is the control system to uncertainties in input parameters and what are their effects on the overall mission performance?

To answer these questions, the ascent and re-entry trajectory of hypersonic spaceplanes shall be studied. In particular, a simulation tool is required to simulate the vehicle's motion and its performance in a accurate way. This tool needs to be designed on the basis of the mathematical representation of the vehicle and its trajectory, so that the influence of the system parameters on the performance can be identified. Moreover, a control system that is able to realise the desired commands and counteract any perturbation acting on the system shall be integrated in the simulator.

Following the logical flow of the applied methodology, the report is structured in 8 chapters. It commences with the present introduction. In Chapter 2 the reference vehicle and reference mission are detailed, providing the mission and system requirements. The flight-environment characteristics, such as the Earth's shape and gravitational field, atmosphere, wind and turbulence models are introduced in Chapter 3. Chapter 4 details the flight mechanics that governs the hypersonic flight of a spaceplane. This is essential to design a simulator of the spacecraft motion. The method to analyse the spaceplane stability characteristics are defined in Chapter 5. Also, this methodology is applied to identify and investigate the eigenmotion properties, trim capabilities, and the flying-qualities. A control system is generally designed starting from a simple method and, afterwards, improving it with a more advanced system. The Linear Quadratic Regulator (LQR) and the Simple Adaptive Control (SAC) are chosen as benchmark and more advanced controller, respectively. The control laws and the design of these control systems are described in Chapter 6, where the baseline control system is designed. Chapter 7 presents how, starting from the baseline control design, the robust design methodology is used to identify the design that is as insensible to perturbations and uncertainties of the input parameters as possible. This is done by means of a double-loop sensitivity analysis. Once the robust control-system design is identified, controllability characteristics are analysed, followed by the investigation of the system's capability to respond to atmospheric disturbances. Finally, Chapter 8 summarises the entire picture of the research outcomes discussed in the previous chapters, and proposes ideas for future research developments. Supplementary material is reported in Appendices A and B. In Appendix A, the state-space model of the linearised equations of motion (EoM) is detailed. Details about the software architecture of the flight simulator are provided in Appendix B, where also the verification of the implemented models is conducted. 


\section{2}

\section{Mission \& System Definition}

Flying characteristics and controllability have been studied since the early 1900s when the Wright brothers achieved the first manned flight with a motorised aircraft. The Wright brothers' objective was to ensure appropriate mission performance and flight safety. Although nowadays the increased computational capability has led to improved tools and methods for analysing flying qualities and controllability characteristics, the scope of studying them has remained virtually unchanged.

Past and recent missions and studies have been conducted about flying qualities and controllability of hypersonic spaceplanes. However, the analysis shows that there are not so many documents detailing this topic, which leads us to understand that this remains an open field where, consequently, a deeper analysis could represent a great contribution to the scientific community. The reference vehicle is selected and its characteristics are specified in 2.1. In Sections 2.2 the reference mission is presented. From the thesis objectives, identified in Section 1.2, and the specifications of the reference vehicle and mission, the mission and system requirements of this thesis project are specified in Section 2.3.

\subsection{Reference Vehicle}

In Section 1.1.2, the WCC, FSS-1 and vehicle by GMV are proposed as reference vehicle, indicating their main characteristics. To achieve the objective of the mission, it is important that the database of the reference vehicle is complete and consistent, and that the vehicle can be controlled along its flight. Thus, the selection criteria to identify the reference vehicle are:

1. completeness of the aerodynamic, propulsion, and mass databases;

2. consistency of the databases;

3. controllability of the vehicle.

Although the launch vehicle proposed by GMV has an extensive propulsion database, the aerodynamic database is not complete. This is likely not enough to conduct a proper and complete stability analysis, and flying-quality assessment. Moreover, its control surfaces are only two elevons that can generate pitch and roll moments, when deflected symmetrically and asymmetrically, respectively. So, the yaw motion can not be controlled. Differently, the aerodynamic database, as well as propulsion and mass databases, of both WCC and FSS- 1 are complete. However, FSS- 1 seems to be convenient for the thesis mission, since its mass and propulsion models are analysed to a higher quality. The reason is that the WCC has a total mass (including fuel) of $140 \mathrm{t}$, a dry mass that is around $50 \mathrm{t}$ at take-off, and moments of inertia that are 10 times lower than FSS-1. This means that the WCC is potentially more affected by perturbations and disturbances resulting in a lower overall stability. If one reminds that Space Shuttle orbiter has a dry weight of about $40 \mathrm{t}$ along its reentry trajectory, where it only descends. Thus, it can be concluded that the WCC's mass properties are likely not appropriate for a stable vehicle. Of course, it is possible to recalculate the mass database, scaling it to the correct mass properties, but it would require additional work and effort. Moreover, it could result in inconsistencies between the databases.

FSS-1 is the vehicle that better suits the selection criteria since, not only its behaviour during flight can be quite representative of the hypersonic spaceplanes characteristics, but also it has complete and consistent 

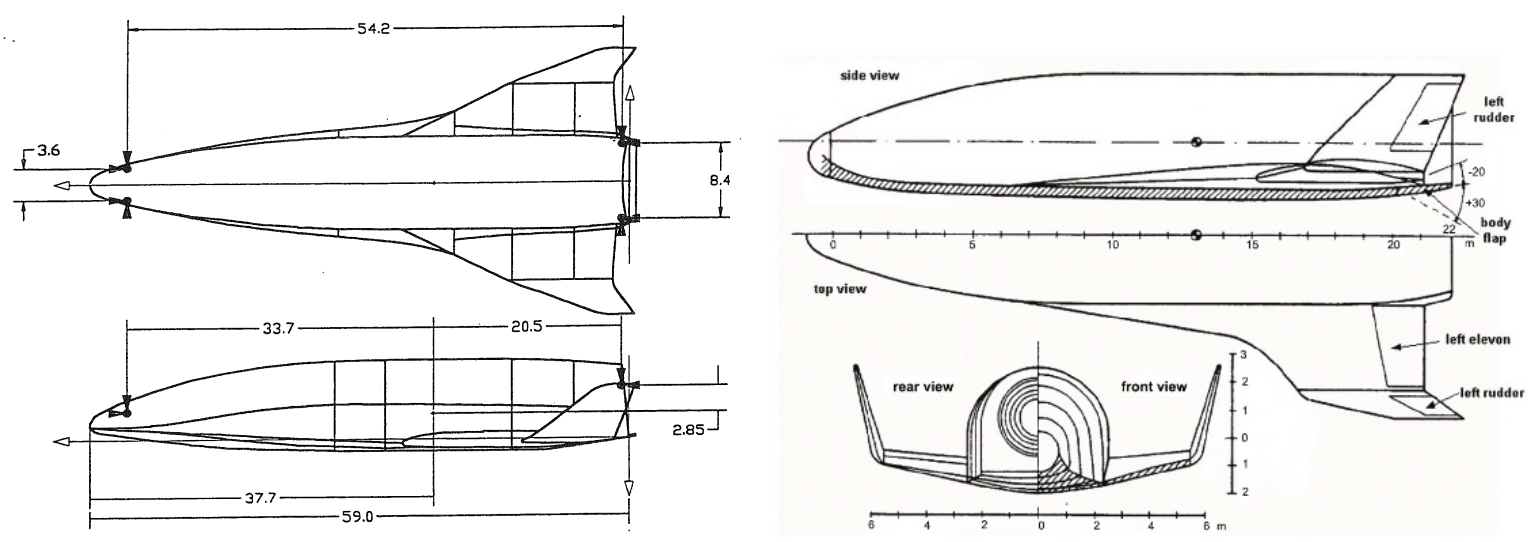

Figure 2.1: Reference geometry of FSS-1 (left) [Daimler-Benz Aerospace, 1998] and HORUS (right), adapted from [MBB, 1988].

Table 2.1: Main characteristics of FSS-1 reference vehicle and HORUS [Daimler-Benz Aerospace, 1998].

\begin{tabular}{||lcc||}
\hline Dimensions & FSS-1 & HORUS \\
Fuselage Length [m] & 59 & 23 \\
Total Length (incl. nozzles) $[\mathrm{m}]$ & 62.2 & 25 \\
Maximum fuselage width [m] & 10.35 & 5.4 \\
Maximum fuselage height [m] & 10.33 & 4.5 \\
Aerodynamic Ref.Area [m $\left.{ }^{2}\right]$ & 820 & 110 \\
Wing Chord [m] & 30 & 23 \\
Wing Span [m] & 29.4 & 13 \\
Wing Area [m $\left.{ }^{2}\right]$ & 270 & 110 \\
Winglet Area [m²] & 84 & 24 \\
\hline
\end{tabular}

databases and both the longitudinal and lateral-directional motion can be controlled during fight. Moreover, an important advantage is the availability of the HORUS aerodynamic database [MBB, 1988] that can support the analysis by providing the aerodynamic force and moments coefficients as a function of Mach number, angle of attack, and control-surface deflection. Also, as discussed before, choosing FSS-1 as reference vehicle allows to use HORUS as benchmark for the software verification and for the stability and flying-quality analysis.

During this thesis work, the FSS-1 design defined by Daimler-Benz Aerospace [1998] is examined. The analyses that will be conducted will identify the capabilities of the FESTIP design in terms of stability and controllability and, at the same time, the shortcomings will be identified and solutions will be proposed to improve the design for future works. The features of the reference vehicle are described in the following. Figure 2.1 illustrates the FSS-1 and HORUS geometry, showing their similarities, and Table 2.1 compares their main geometric characteristics. These similarities allow for using the aerodynamic database of HORUS, which is fully available, as the FSS- 1 aerodynamic database. However, a more careful investigation shows that the FSS- 1 does not simply derive from HORUS by multiplying it with a scale factor, as some dimensions were modified by Daimler-Benz Aerospace [1998] for trimmability and controllability reasons. As a consequence, the database should be corrected so that the differences in the FSS-1 geometry can be taken into account, as will be discussed in Section 2.1.1.

The control surfaces of the reference vehicle are:

- left and right elevons: the deflection angle, respectively $\delta_{w, l}$ and $\delta_{w, r}$, are positive downward;

- left and right rudders: the deflection angle, respectively $\delta_{r, l}$ and $\delta_{r, r}$, are positive outward;

- body flap: the deflection angle, $\delta_{b}$, is positive downward;

where the subscripts " $r$ " and " $l$ " are used to indicate the right and the left elevon/rudder, respectively. Note that the elevons provide the functions of both elevator (if symmetrically deflected) and aileron (if asymmetrically deflected). Thus, the elevator deflection, $\delta_{e}$, is equal to the symmetric combination of the left and right elevon deflections, while the aileron deflection, $\delta_{a}$, is defined as the asymmetric combination of the two elevon 
deflections, i.e.:

$$
\delta_{e}=\frac{\delta_{w, l}+\delta_{w, r}}{2}, \quad \delta_{a}=\frac{\delta_{w, l}-\delta_{w, r}}{2}
$$

The rudders can be deflected only outward and only one rudder at a time is moved for yaw control. Thus, the rudder deflection, $\delta_{r}$, is defined as:

$$
\delta_{r}= \begin{cases}\delta_{r, l}, & \text { if } \delta_{r, l} \geq 0 \\ -\delta_{r, r}, & \text { if } \delta_{r, r} \geq 0\end{cases}
$$

It was found by Mooij [1998a] that, at least during re-entry, the rudders are very weak and, consequently, they are not sufficient to fully control the vehicle. Thus, it is likely that its activity needs to be supported by other actuators, such as the engine gimbal.

The vehicle can be trimmed by the body flap and, when it does not suffice, it can be supported by the elevons and engine gimbal. The longitudinal motion can be controlled by commanding the elevator, while the lateral motion can be controlled by commanding the aileron and rudder. Moreover, the main engines can be used to support the control surfaces in controlling the vehicle by changing their orientation so that the thrust vector points in the desired direction. This method is known as thrust vectoring control (TVC). During re-entry, a similar contribution to attitude control is provided by the Reaction Control System (RCS).

\subsubsection{Aerodynamic System}

Originally, the FSS-1 configuration was derived by linearly scaling the HORUS vehicle, the second stage of the TSTO spaceplane Sänger II. As illustrated in Figure 2.1, their geometry is very similar, which suggests that the HORUS aerodynamic database could be used for the FSS-1 flight simulations and analysis. However, Daimler-Benz Aerospace [1998] slightly modified the FSS-1 configuration in the interest of stability and control. One of the modification is the resizing of the wings ( $10 \%$ reduction of the exposed wing) to optimize the vehicle that, with the same shape of HORUS, has to be capable to perform also the vertical take-off and ascending flight to orbit. Indeed, a wing reduction is linked to a decrease of the aerodynamic perturbations. The wings are also backward shifted by $2.5 \mathrm{~m}$ to improve trimmability and controllability. The physical reason is that the aerodynamic reference centre of the wings is shifted with respect to the CoM, inducing larger aerodynamic moments with the same control-surface deflection due to longer lever arms. Also, in this way smaller deflections of the body and wing flaps are required to trim. Finally, to get less base drag, the fuselage height is reduced. These modifications result in an improved lift-to-drag ratio and improved directional stability and controllability.

The changes in FSS-1 geometry have an impact on the aerodynamics of the vehicle, thus we would expect that its aerodynamic database presents some differences when compared to the HORUS database. The FESTIP documentation does not offer a complete aerodynamic database of FSS-1, but only presents some figures of lift, drag and pitch coefficients as a function of angle of attack and Mach number that could be useful to analyse the database. Contrary, the HORUS technical reports [MBB, 1988] provide a complete aerodynamic database. Thus, it is convenient to use the HORUS aerodata and correct them so that the aerodynamic coefficients resemble as much as possible the FSS-1 ones, by taking advantage of the plots that are presented by Daimler-Benz Aerospace [1998].

The HORUS aerodynamic database is based on the assumptions that the vehicle is a rigid body, thus no aeroelasticity phenomena are modelled, and that there are no interference effects of the flaps. The aerodynamic coefficients are a function of the Mach number $M$, angle of attack $\alpha$, altitude $h$, sideslip angle $\beta$, and deflection angles that derive from the motion of the vehicle's control surfaces.

The definition of the aerodynamic coefficients is the following:

$$
\begin{aligned}
& C_{D}=C_{D_{0}}+\Delta C_{D_{r, l}}+\Delta C_{D_{w, l}}+\Delta C_{D_{b}}+\Delta C_{D_{w, r}}+\Delta C_{D_{r, r}}-\Delta C_{D_{h}} \\
& C_{S}=\Delta C_{S_{r, l}}+\Delta C_{S_{w, l}}+\Delta C_{S_{w, r}}+\Delta C_{S_{r, r}}+\left[\left(\frac{\partial C_{S}}{\partial \beta}\right)_{0}+\Delta\left(\frac{\partial C_{S}}{\partial \beta}\right)_{w, l}+\Delta\left(\frac{\partial C_{S}}{\partial \beta}\right)_{w, r}\right] \beta \\
& C_{L}=C_{L_{0}}+\Delta C_{L_{w, l}}+\Delta C_{L_{b}}+\Delta C_{L_{w, r}} \\
& C_{l}=\Delta C_{l_{w, l}}+\Delta C_{l_{w, r}}+\left(\frac{\partial C_{S}}{\partial \beta}\right)_{0} \cdot \beta \\
& C_{m}=C_{m_{0}}+\Delta C_{m_{w, l}}+\Delta C_{m_{b}}+\Delta C_{m_{w, r}} \\
& C_{n}=\Delta C_{n_{r, l}}+\Delta C_{n_{w, l}}+\Delta C_{n_{w, r}}+\Delta C_{n_{r, r}}+\left[\left(\frac{\partial C_{n}}{\partial \beta}\right)_{0}+\left(\frac{\partial C_{n}}{\partial \beta}\right)_{r, l}+\Delta\left(\frac{\partial C_{n}}{\partial \beta}\right)_{r, r}\right] \beta
\end{aligned}
$$


Table 2.2: Aerodynamic coefficients [MBB, 1988].

\begin{tabular}{||ccc||}
\hline Drag coefficients & Side-force coefficients & Lift coefficients \\
\hline$C_{D_{0}}=f(M, \alpha)$ & $\Delta C_{S_{r, l}}=f\left(M, \alpha, \delta_{r, l}\right)$ & $C_{L_{0}}=f(M, \alpha)$ \\
$\Delta C_{D_{r, l}}=f\left(M, \alpha, \delta_{r, l}\right)$ & $\Delta C_{S_{w, l}}=f\left(M, \alpha, \delta_{w, l}\right)$ & $\Delta C_{L_{w, l}}=f\left(M, \alpha, \delta_{w, l}\right)$ \\
$\Delta C_{D_{w, l}}=f\left(M, \alpha, \delta_{w, l}\right)$ & $\Delta C_{S_{w, r}}=f\left(M, \alpha, \delta_{w, r}\right)$ & $\Delta C_{L_{b}}=f\left(M, \alpha, \delta_{b}\right)$ \\
$\Delta C_{D_{b}}=f\left(M, \alpha, \delta_{b}\right)$ & $\Delta C_{S_{r, r}}=f\left(M, \alpha, \delta_{r, r}\right)$ & $\Delta C_{L_{w, r}}=f\left(M, \alpha, \delta_{w, r}\right)$ \\
$\Delta C_{D_{w, r}}=f\left(M, \alpha, \delta_{w, r}\right)$ & $\left(\frac{\partial C_{S}}{\partial \beta}\right)_{0}=f(M, \alpha)$ & \\
$\Delta C_{D_{r, r}}=f\left(M, \alpha, \delta_{r, r}\right)$ & $\Delta\left(\frac{\partial C_{S}}{\partial \beta}\right)_{w, l}=f\left(M, \alpha, \delta_{w, l}\right)$ & \\
$C_{D_{h}}=f(M, h)$ & $\Delta\left(\frac{\partial C_{S}}{\partial \beta}\right)_{w, r}=f\left(M, \alpha, \delta_{w, r}\right)$ & \\
\hline \hline Roll coefficients & Pitch coefficients & Yaw coefficients \\
\hline$\Delta C_{l_{w, l}}=f\left(M, \alpha, \delta_{w, l}\right)$ & $C_{m_{0}}=f(M, \alpha)$ & $\Delta C_{n_{r, l}}=f\left(M, \alpha, \delta_{r, l}\right)$ \\
$\Delta C_{l_{w, r}}=f\left(M, \alpha, \delta_{w, r}\right)$ & $\Delta C_{m_{w, l}}=f\left(M, \alpha, \delta_{w, l}\right)$ & $\Delta C_{n_{w, l}}=f\left(M, \alpha, \delta_{w, l}\right)$ \\
$\left(\frac{\partial C_{l}}{\partial \beta}\right)_{0}=f(M, \alpha)$ & $\Delta C_{m_{b}}=f\left(M, \alpha, \delta_{b}\right)$ & $\Delta C_{n_{w, r}}=f\left(M, \alpha, \delta_{w, r}\right)$ \\
& $\Delta C_{m_{w, r}}=f\left(M, \alpha, \delta_{w, r}\right)$ & $\Delta C_{n_{r, r}}=f\left(M, \alpha, \delta_{r, r}\right)$ \\
& & $\left(\frac{\partial C_{n}}{\partial \beta}\right)_{0}=f(M, \alpha)$ \\
& & $\Delta\left(\frac{\partial C_{n}}{\partial \beta}\right)_{r, l}=f\left(M, \alpha, \delta_{r, l}\right)$ \\
& & $\Delta\left(\frac{\partial C_{n}}{\partial \beta}\right)_{r, r}=f\left(M, \alpha, \delta_{r, r}\right)$
\end{tabular}

Table 2.3: Symmetry conditon of left and right elevons and rudders [MBB, 1988].

\begin{tabular}{||cccc||}
\hline $\begin{array}{c}\text { Zero derivatives } \\
\text { right elevon }\end{array}$ & $\begin{array}{c}\text { First derivative } \\
\text { right elevon }\end{array}$ & $\begin{array}{c}\text { Zero derivatives } \\
\text { right rudder }\end{array}$ & $\begin{array}{c}\text { First derivative } \\
\text { right rudder }\end{array}$ \\
\hline$C_{D_{w, r}}=C_{D_{w, l}}$ & $\left(\frac{\partial C_{S}}{\partial \beta}\right)_{w, r}=\left(\frac{\partial C_{S}}{\partial \beta}\right)_{w, l}$ & $C_{D_{r, r}}=C_{D_{r, l}}$ & $\left(\frac{\partial C_{n}}{\partial \beta}\right)_{r, r}=\left(\frac{\partial C_{n}}{\partial \beta}\right)_{r, l}$ \\
$C_{S_{w, r}}=-C_{S_{w, l}}$ & & $C_{S_{r, r}}=-C_{S_{r, l}}$ & \\
$C_{L_{w, r}}=C_{L_{w, l}}$ & & $C_{n_{r, r}}=-C_{n_{r, l}}$ & \\
$C_{l_{w, r}}=-C_{l_{w, l}}$ & & & \\
$C_{m_{w, r}}=C_{m_{w, l}}$ & & & \\
$C_{n_{w, r}}=-C_{n_{w, l}}$ & & & \\
\hline
\end{tabular}

where the aerodynamic force coefficients are the drag coefficient $C_{D}$, the side-force coefficient $C_{S}$, and the lift coefficient $C_{L}$, while the aerodynamic moment coefficients are the roll coefficient $C_{l}$, the pitch coefficient $C_{m}$, and the yaw coefficient $C_{n}$. The functional arguments of the components of the aerodynamic coefficients are listed in Table 2.2. The two elevons and the two rudders are located symmetrically with respect to the XZ-plane of the vehicle. Since they present the same shape, the components of the aerodynamic coefficients of the right actuator are the same of the left one, while others are equal in module and opposite in sign, as presented in Table 2.3. This is known as the symmetry condition.

Given the summed nature of the aerodynamic coefficients, the aerodynamic database is characterised by the flatness property. Indeed, for a given Mach number and angle of attack the moment contributions along each axis of the combined actuators can be represented by a piece-wise linear surface in 3D space, where each linear plane-segment of the surface is flat. This concept can be easily understood if we consider that, for example, the gradient of the pitch moment coefficient with respect to left elevon deflection is independent of the right elevon deflection, since it gives a constant contribution to the moment and it disappears in the derivative. This is generally true for all the moment coefficients and actuator contributions. This property will be used at advantage to model the moment allocator, as will be explained in Section 6.4. 
The aerodynamic coefficients allow for calculating the aerodynamic forces and moments:

$$
\begin{aligned}
D & =C_{D} \bar{q} S_{r e f} \\
S & =C_{S} \bar{q} S_{r e f} \\
L & =C_{L} \bar{q} S_{r e f} \\
\mathscr{L} & =C_{l} \bar{q} S_{\text {ref }} b_{\text {ref }} \\
\mathscr{M} & =C_{m} \bar{q} S_{\text {ref }} c_{\text {ref }} \\
\mathscr{N} & =C_{n} \bar{q} S_{\text {ref }} b_{\text {ref }}
\end{aligned}
$$

where the numerical values for the reference geometry are [Daimler-Benz Aerospace, 1998]:

$$
S_{\text {ref }}=820 \mathrm{~m}^{2}, \quad b_{\text {ref }}=29.4 \mathrm{~m}, \quad c_{\text {ref }}=30 \mathrm{~m}
$$

If one compares the $\mathrm{C}_{L}$ and $\mathrm{C}_{D}$ plots by Daimler-Benz Aerospace [1998] with the ones obtained from the HORUS database, it is revealed that the $C_{L}$ and $C_{D}$ from the HORUS database for a trimmed condition with body flap deflection $\delta_{b}=10^{\circ}$ correlates at first sight with those of FSS-1. However, by observing them more carefully, it can be seen that, although they present a similar shape, they deviate with a certain factor that changes as function of the angle of attack and Mach number. This deviation could be due to the modifications of the vehicle configuration with respect to HORUS that have been discussed before. Single values of the aerodynamic coefficients are as important as the shape of the curve, since the derivatives of the aerodynamic coefficients are essential to investigate the flying qualities, stability and controllability characteristics, as will be presented in Chapter 5 .

It follows that the aerodynamic database needs to be adjusted to account for the different configuration of the vehicle, such that it will be representative of its behaviour. This is done by means of a grahical method. It was noticed that the differences between the curves vary depending on the range of angle of attack and on the Mach number. So, the curves relative to certain Mach numbers needs to be considered one at a time. For each angle-of-attack range of each curve, the geometry of the HORUS coefficient curves is studied and compared to the one of FSS-1. In this way, it is possible to identify to what extent the shape and the slope of the curve needs to be modified, so that the two curves match. Figure 2.2 illustrates the obtained coefficients as a function of Mach number and angle of attack, showing the obtained matching of the coefficients curves with the one presented by Daimler-Benz Aerospace [1998]. Thus, the adapted HORUS aerodynamics is adequate to be representative of the FSS-1 behaviour.

However, it should be mentioned that a certain margin of uncertainties on the values of the coefficients and the inclination of the curves has to be considered. This is due to the fact that the FSS-1 aerodata are given in form of plots, which do not constitute a very accurate source of data. Furthermore, another source of error can be identified in the fact that the data are derived from computer simulations only, without being confirmed by either wind-tunnel data or flight data. However, although some uncertainties can affect the data, this graphical method was useful to adapt the HORUS aerodynamics to be representative of the FSS-1 behaviour. The same procedure is followed for the other plots that are offered by Daimler-Benz Aerospace [1998]. Figure 2.3 shows the lift-to-drag ratio as a function of the angle of attack and Mach number and the pitch coefficient as a function of the angle of attack and body-flap deflection for Mach 15.

Plots of the side-force coefficient, the roll coefficient, and the yaw coefficient are not available. Thus, these coefficients can be corrected by considering what are the effects of the configuration changes on the aerodynamics. The wing resizing affects the lift and drag coefficients that we have already adjusted. The other main changes that remain to be analysed are the reduction of the side area of the vehicle due to the reduction of the fuselage height and the backward shift of the wings.

The reduction of the fuselage height results in a smaller side area of the vehicle. If we calculate the pressure distribution on the fuselage surface to compute the side force, we get that the side force is proportional to this lateral surface. So, with a smaller lateral surface a smaller side force is generated. Figure 2.4 shows the side view of our reference vehicle. Since we are only interested in the difference in side surface due to the height reduction, we can approximated the shape of FSS-1 side with a right trapezoid (red contour illustrated in Figure 2.4), whose surface area is calculated as $S=\frac{\left(b_{1}+b_{2}\right) h}{2}$. Here, $b_{1}$ and $b_{2}$ are the longer and the shorter bases, respectively, and $h$ is the height. Consequently, the side force coefficient can be corrected by scaling it for the factor that can be estimated as:

$$
\frac{S_{\text {side, new }}}{S_{\text {side }, \text { old }}}=\frac{\left(b_{1}+b_{2}\right) h_{\text {new }} / 2}{\left(b_{1}+b_{2}\right) h_{\text {old }} / 2}=\frac{h_{\text {new }}}{h_{\text {old }}}=\frac{10.33}{11.54}=0.9
$$



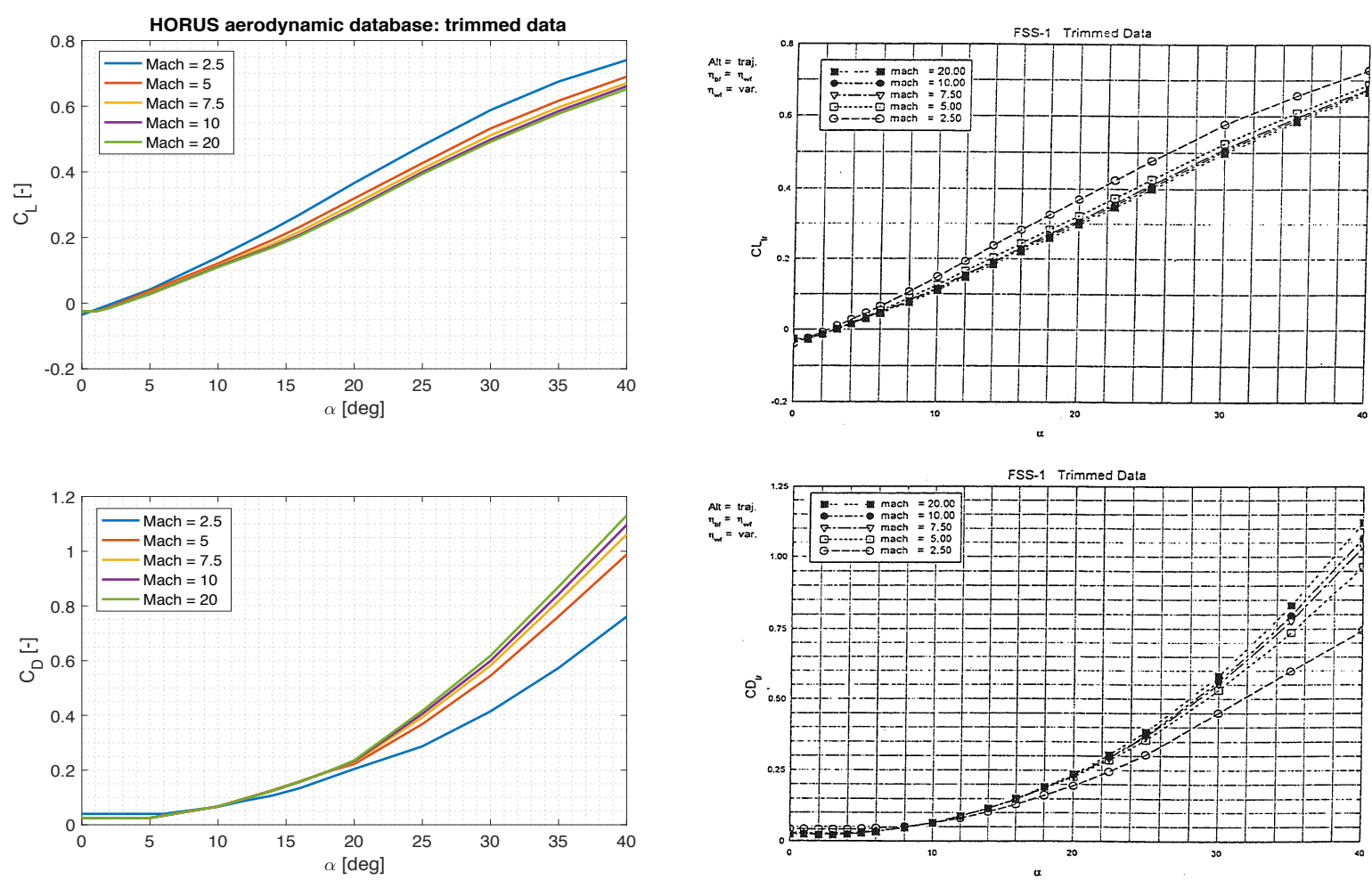

Figure 2.2: Trimmed aerodata lift and drag coefficients, $C_{L}$ and $C_{D}$, as a function of angle of attack and Mach number. Left: corrected HORUS aerodata, right: FSS-1 aerodata [Daimler-Benz Aerospace, 1998].
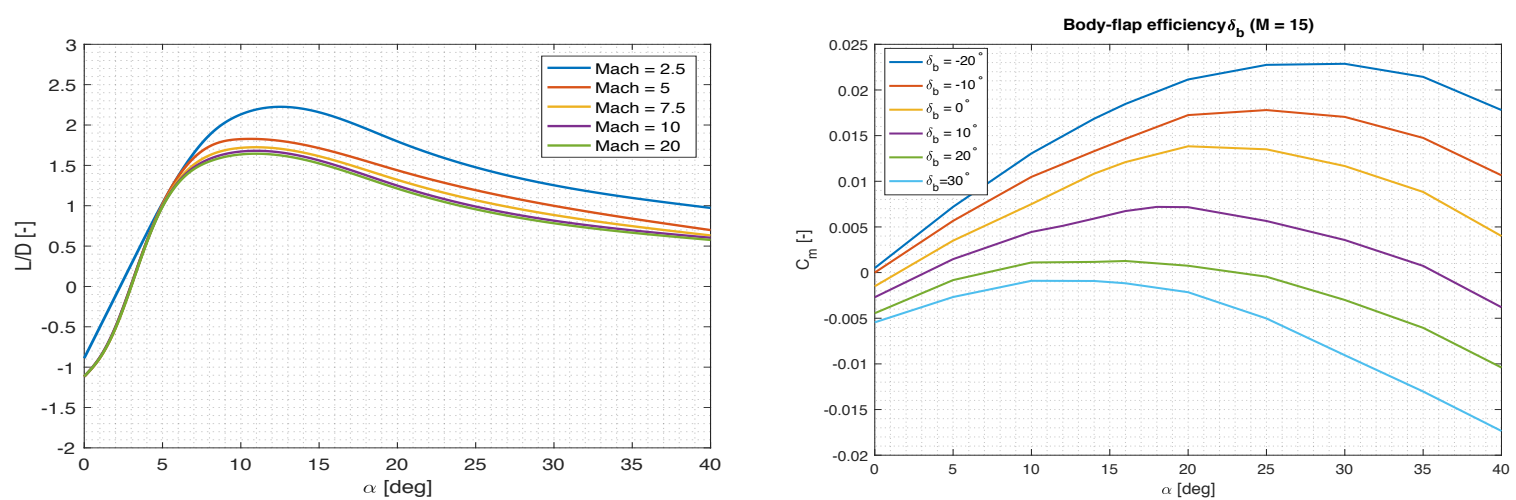

Figure 2.3: Adjusted trimmed aerodata lift-to-drag ratio, L/D, as a function of angle of attack and Mach number (left). Adjusted trimmed aerodata pitch coefficients, $\mathrm{Cm}$, as a function of angle of attack and body flap deflection (right).

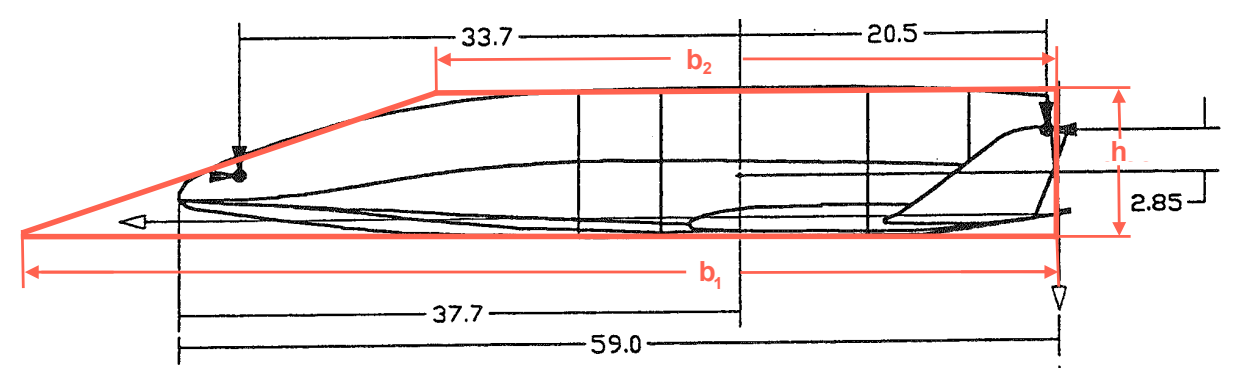

Figure 2.4: FSS-1 side view approximated with a right trapezoid to compute the scaling. 


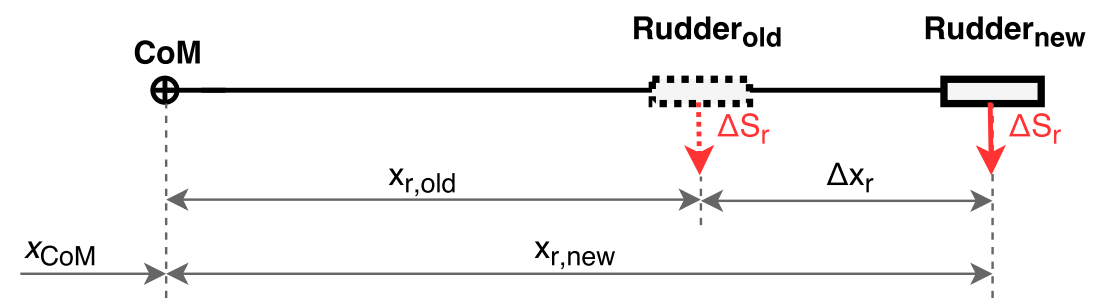

Figure 2.5: FSS-1 rudder $x$-position variation.

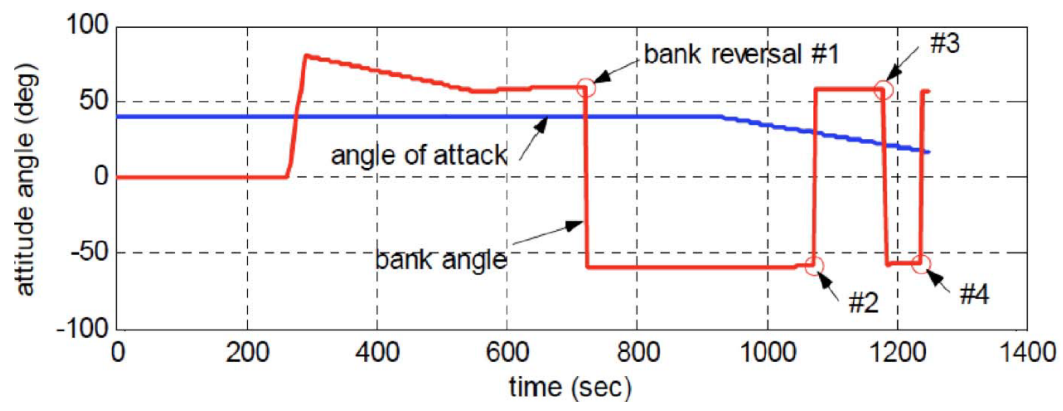

Figure 2.6: HORUS re-entry trajectory: nominal control history Mooij [2015].

The backward shift of the wings influences the yaw moment since the contribution of the side forces due to the rudders is different, because of the different lever arms with respect to the CoM. As Figure 2.5 shows, to correct the yaw-moment coefficient and to adjust the efficiency of the rudder with respect to the CoM, the yaw moment due to the side force induced by the rudder needs to be corrected as follows:

$$
\Delta \mathscr{N}_{r, \text { new }}=\Delta S_{r} x_{r, \text { new }}=\Delta S_{r}\left(x_{r, \text { old }}+\Delta x\right)=\Delta S_{r} x_{r, \text { old }}+\Delta S_{r} \Delta x=\Delta \mathscr{N}_{r, \text { old }}+\Delta S_{r} \Delta x
$$

where $\Delta x=2.5 \mathrm{~m}$ is the shift of the wing axial position with respect to its original position.

Finally, the roll moment coefficient $C_{l}$ is not affected, to a first approximation, neither by the wing shift since it is in $x$-direction, nor by the reduction of the fuselage height. The resizing of the wings would have an effect on $C_{l}$ if the ailerons are resized, too, since it would generate a slightly different pressure distribution of the flow. However, the FESTIP documentation does not present how the wings are resized and if and how also the ailerons are reshaped. Thus, it could be assumed that, whether the ailerons are reshaped or not, it is a second-order effect so that the roll moment coefficient can be kept the same as for the HORUS configuration.

\subsubsection{Aero-data Verification}

The verification of the adapted FSS-1 aerodynamic database is performed. The re-entry trajectory flown by HORUS was taken as reference trajectory and it is investigated whether FSS-1, with its own characteristics and aerodynamic properties, is able to fly the same trajectory. This should be verified provided that FSS-1 follows the same profiles of angle of attack and bank angle of HORUS, and starting from the same initial conditions, i.e., $h=122 \mathrm{~km}, V=7435.5 \mathrm{~m} / \mathrm{s}, \gamma=-1.43^{\circ}$ [Mooij, 1998b]. The results that are presented are generated by using the flight simulator detailed in Appendix B, where the equations of motion of the vehicle presented in Chapter 4 are integrated.

The nominal control history of the angle of attack and bank angle, shown in Figure 2.6, are defined for HORUS through an optimization process so that the constraints on heat flux and g-load are respected [Mooij, 2015]. The results of the trajectory simulation are presented in the $h$-V diagram, given in Figure 2.7. As it is shown, the trajectory followed by FSS-1 is different from HORUS trajectory. This can be explained by considering the different aerodynamic configurations of the vehicles.

Indeed, FSS-1 is characterized by higher weight and lift, whose combination lets FSS-1 reach a slightly lower altitude at the beginning followed by a skipping motion, where the vehicle is pushed up by the atmosphere, performing a short upward flight inside the atmosphere without leaving it. The reason is that, as illustrated in Figure 2.8, the flight-path angle at the first local low of the $h-V$ diagram is less negative with respect to HORUS, which confirms that FSS- 1 is performing a steeper entry in the atmosphere. Overall, this fact affects the remaining portion of flight: the deeper dive into the atmosphere induces a more rapid dissipation of energy resulting in lower velocities at corresponding altitudes and, due to the skipping, the flight time required to reach 

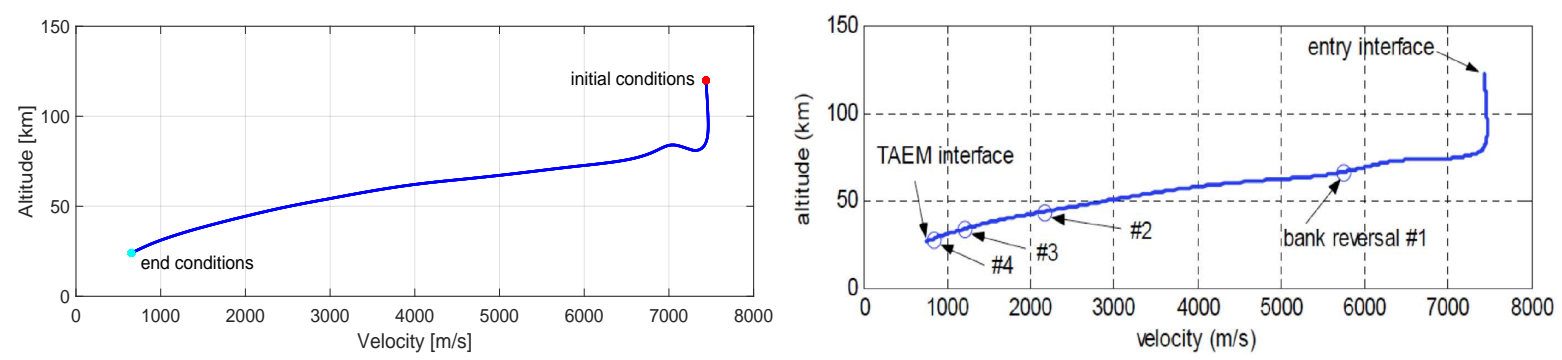

Figure 2.7: HORUS re-entry trajectory: altitude-velocity profile. Left: simulation of FSS-1 re-entry trajectory; right: HORUS re-entry trajectory by [Mooij, 2015].
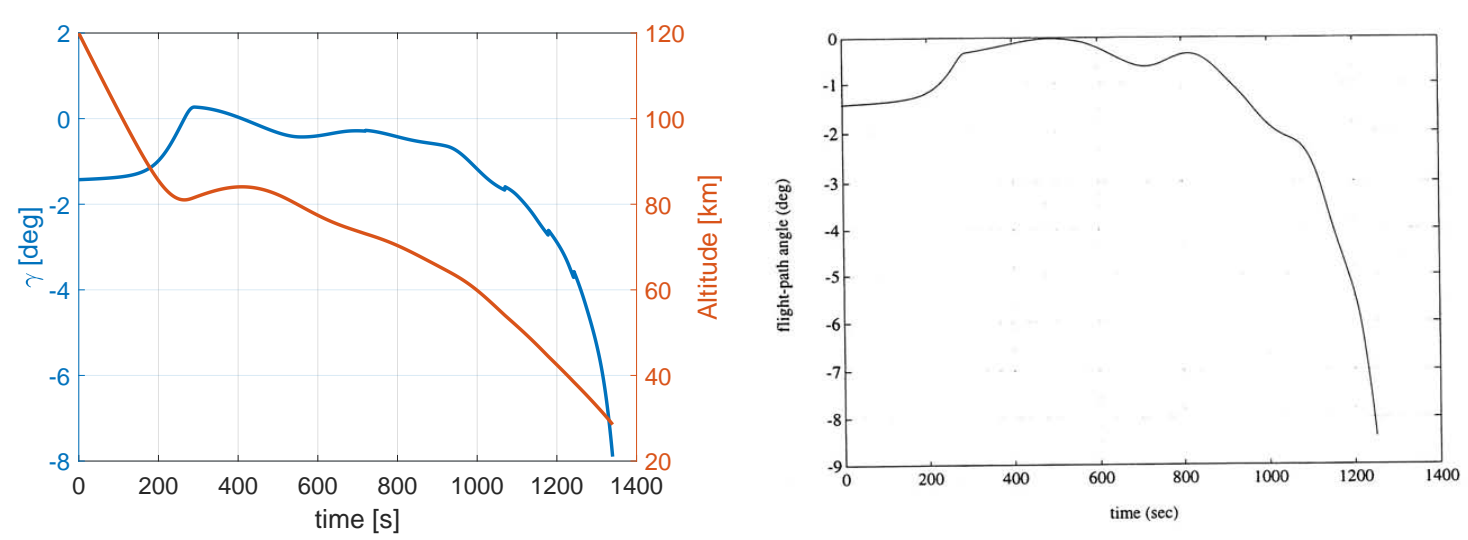

Figure 2.8: Flight-path angle and altitude of FSS-1 re-entry trajectory (left) and flight-path angle of HORUS re-entry trajectory (right) as function of time.

the same end conditions is greater of about $100 \mathrm{~s}$ than for a regular gliding flight, performed by HORUS. The skipping motion can be avoided and the final conditions can be reached if the larger weight and lift are compensated with a higher, i.e., less negative, initial flight-path angle. For instance, increasing $\gamma$ by $0.2^{\circ}\left(\gamma=-1.23^{\circ}\right)$ is already sufficient to avoid the skipping. This analysis ensures that the FSS-1 aerodynamics is appropriate for the vehicle, since it is able to fly the HORUS trajectory with some minor difference justified by the discrepancy in aerodynamic configuration.

Moreover, it is proved by the successive trim analysis (Section 5.4.2), that the aerodynamic data is so that the trim conditions of the points of interest along the FSS-1 trajectory are quite similar to those presented by Daimler-Benz Aerospace [1998]. This ensures that the modifications to the aerodynamic database are adequate, to a first approximation, to be representative of the FSS-1 behaviour along its hypersonic trajectory.

\subsubsection{Propulsion System}

Daimler-Benz Aerospace [1998] provides the characteristics of the propulsion system of FSS-1. It has 8 engines ( 4 boosters and 4 sustainer) with fixed bell nozzles using the cryogenic propellent $\left(\mathrm{LO}_{2} / \mathrm{LH}_{2}\right)$ with a oxidizer-fuel $(\mathrm{O} / \mathrm{F})$ mixture ratio variable between 6.0 and 7.5. They are designed to have a high thrust level of $1800 \mathrm{kN}$, since they have to provide the thrust necessary to move the vehicle from the Earth's surface to the vacuum of space during the ascent flight. This high thrust level can be reached thanks to a moderately high combustion chamber pressure of 180 bar, and a very high specific impulse. Engine throttle and thrust vectoring can be used during the ascent flight to guide and control the vehicle. The liquid-propellant rockets can be throttled by means of valves that regulate the amount of fuel and oxidizer flowing to the combustion chamber. In this way, it is possible to control and vary the thrust level during flight. Figure 2.9 shows the locations of the 8 engines in a rear view of the vehicle and identifies the centre of thrust. The main parameters of the FSS-1 propulsion system are presented in Table 2.4 .

A schematic representation of a rocket engine (Figure 2.10) can help to briefly describe the operating principles of a cryogenic rocket engine, on the basis of the theory exposed by Turner [2005]. A cryogenic rocket engine generally comprises of a combustion chamber, a nozzle, propellent tanks, and the means through which the propellants are delivered to the combustion chamber. Thanks to the high pressure in the tanks, the fuel and the oxidizer reach the combustion chamber as a fine spray so that they can rapidly mix. Thereafter, the com- 
Table 2.4: Main engine characteristics of our reference vehicle FSS-1 [Daimler-Benz Aerospace, 1998].

\begin{tabular}{||lc||}
\hline FSS-1 Engine Characteristics & \\
Thrust Level [kN] & 1800 \\
Chamber Pressure [bar] & 150 \\
$\mathrm{I}_{s p}$ Booster sea level [s] & 389 \\
$\mathrm{I}_{s p}$ Sustainer sea level [s] & 345 \\
I $s p$ Sustainer vacuum [s] & 448 \\
Nozzle Area Ratio Booster [-] & 25 \\
Nozzle Area Ratio Sustainer [-] & 66 \\
T/W Ratio Booster sea level [-] & 65.1 \\
T/W Ratio Sustainer sea level [-] & 50.1 \\
T/W Ratio Sustainer vacuum [-] & 65 \\
O/F Mixture Ratio [-] & 6.0 to 7.5 \\
Main Engine Gimbal Angle [-] & $\pm 4^{\circ}$ \\
\hline T/W Ratio: thrust-to-weight ratio & \\
\hline
\end{tabular}

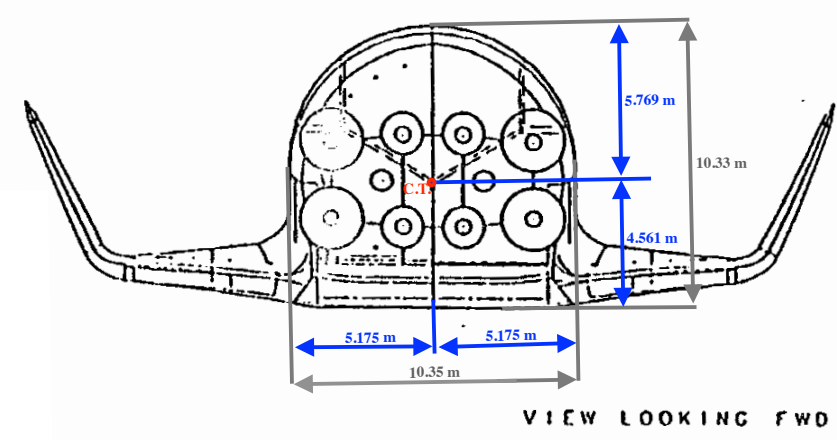

Figure 2.9: FSS-1 rear view: engine locations and centre of thrust (C.T.) [Daimler-Benz Aerospace, 1998].
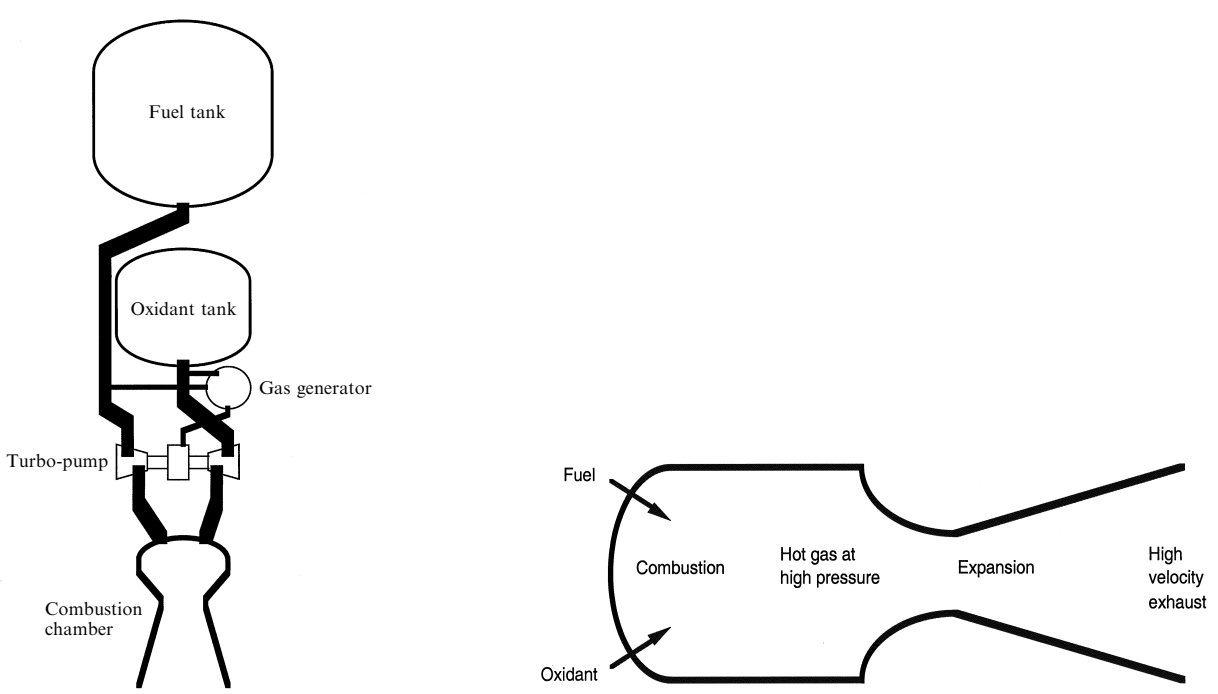

Figure 2.10: Schematic model of the cryogenic rocket engine (left) and of the combustion chamber (right) [Turner, 2005].

bustion is triggered by a safe and reliable igniter. Generally, for $\mathrm{LH}_{2} / \mathrm{LO}_{2}$ engines, it is an electrical spark igniter that is used. The high-pressure hot gas, resulting from the combustion, is then converted into a high-velocity exhaust stream by the expansion nozzle, since the gas expands and cools while passing through the nozzle. The expansion of the hot gas against the wall of the nozzle generates the acceleration of the vehicle. The rate of mass expulsion $\dot{m}$ enters the exit area of the nozzle $A_{e}$ with a velocity $\mathbf{V}_{\mathbf{T}}$ (collinear to the thrust vector $\mathbf{T}$ ) at the centre of mass flow or centre of thrust (CoT), located at a distance $\mathbf{r}_{\mathrm{T}}$ from the CoM of the vehicle.

The TVC carries out, together with the control surfaces, a fundamental function within the control system: they are responsible to provide the control actions that the control logic commands. Varying the direction of the thrust vector, the vehicle attitude can be controlled. The propulsion moments about the CoM are generated varying the thrust elevation and azimuth. As shown in Figure 2.11, the thrust elevation $\epsilon_{T}$ is defined as the angle between the thrust vector and the $X_{B}-Y_{B}$ plane of the body reference frame, $\mathscr{F}_{B}$ (see Section 4.2). The thrust azimuth $\psi_{T}$ is the angle between the projection of thrust force on the $X_{B}-Y_{B}$ plane and the $X_{B}$-axis. It should be noticed that in Figure 2.11, the components of the thrust vector $\mathbf{T}$ with respect to $\mathscr{F}_{B}$ can be calculated:

$$
\begin{aligned}
& T_{x}=T \cos \epsilon_{T} \cos \psi_{T} \\
& T_{y}=T \cos \epsilon_{T} \sin \psi_{T} \\
& T_{z}=T \sin \epsilon_{T}
\end{aligned}
$$

where $T$ is the thrust magnitude. Considering the distance between the CoT and the CoM of the vehicle as $\mathbf{r}_{\mathbf{T}}=\left(x_{T}, y_{T}, z_{T}\right)^{T}$, the propulsion moment can be calculated as $\mathbf{M}_{\mathbf{T}}=\mathbf{r}_{\mathbf{T}} \times \mathbf{T}$, whose cross product can be solved 




Figure 2.11: Thrust vector components with respect to the local body reference frame (with the origin coincident with the centre of thrust (CoT)), with the CoT located at distance $\mathbf{r}_{\mathbf{T}}$ from the CoM.

to give the explicit expressions of the propulsion moment components:

$$
\mathbf{M}_{\mathbf{T}}=\left(\begin{array}{l}
M_{T, x} \\
M_{T, y} \\
M_{T, z}
\end{array}\right)=\left(\begin{array}{l}
T_{z} y_{T}-T_{y} z_{T} \\
T_{x} z_{T}-T_{z} x_{T} \\
T_{y} x_{T}-T_{x} y_{T}
\end{array}\right)
$$

Substituting the expressions of $T_{x}, T_{y}$, and $T_{z}$, it yields:

$$
\begin{aligned}
& M_{T, x}=T y_{T} \sin \epsilon_{T}-T z_{T} \cos \epsilon_{T} \sin \psi_{T} \\
& M_{T, y}=T z_{T} \cos \epsilon_{T} \cos \psi_{T}-T x_{T} \sin \epsilon_{T} \\
& M_{T, z}=T y_{T} \cos \epsilon_{T} \sin \psi_{T}-T x_{T} \cos \epsilon_{T} \cos \psi_{T}
\end{aligned}
$$

Note that $\epsilon_{T}$ is positive when it contributes to a positive change of flight-path angle and $\psi_{T}$ is positive when it contributes to a positive change of heading angle. Although structural limits of the vehicle allows for small maximum and minimum deflection angles of the thrust vector (see Table 2.6), the TVC effectiveness is quite high along the whole ascent flight, differently from the control surfaces whose effectiveness depends on $\bar{q}$, as will be shown by the controllability analysis.

\subsection{Reference Mission}

The FSS-1 optimal trajectory designed by Daimler-Benz Aerospace [1998]. The vehicle performs a powered vertical take-off and ascent to orbit. The booster engine cut-off occurs at a height of $59 \mathrm{~km}$, and main engine cut-off (MECO) at around $102 \mathrm{~km}$ altitude. Finally, after it has completed its orbital mission, it performs the re-entry flight and lands horizontally.

The thesis goal is to study the stability and controllability characteristics of the vehicle in nominal and offnominal (e.g., in presence of atmospheric disturbances) conditions. To completely define the stability properties of the vehicle, the characteristics of motion of the open-loop system shall be studied, together with the trimmability capabilities. Afterwards, the flying qualities can be identified and compared with the MIL-Specs requirements, which are detailed in Chapter 5. Once it is determined whether the vehicle is stable and whether its flying qualities comply with the MIL standards, the need for a control system arises. The control system, chosen mainly on the basis of its robustness, minimum control effort and error, and supporting reference materials, is designed in Chapter 6. The controllability analysis and the response to atmospheric disturbances can be studied to investigate whether the control system is able to compensate for any deficiency of the system and met the MIL specifications. These analyses will be conducted for some key points of the hypersonic portion of flight. In particular, it is chosen to investigate the points where the Mach number is 5, 10, 15, 20 along both the ascent and the re-entry trajectory. In this way a clear picture of the stability and controllability characteristics of hypersonic vehicles can be drawn. The nominal conditions at each point of interest are presented in Table 
Table 2.5: Nominal conditions at the design points along the ascent and re-entry trajectory [Daimler-Benz Aerospace, 1998].

\begin{tabular}{|c|c|c|c|c|c|c|c|c|}
\hline \multirow[t]{2}{*}{ Initial Conditions } & \multicolumn{4}{|c|}{ Ascent } & \multicolumn{4}{|c|}{ Re-entry } \\
\hline & $\mathbf{M}=5$ & $M=10$ & $M=15$ & $\mathbf{M}=\mathbf{2 0}$ & $\mathbf{M}=\mathbf{5}$ & $M=10$ & $M=15$ & $\mathbf{M}=\mathbf{2 0}$ \\
\hline Altitude $[\mathrm{km}]$ & 34.54 & 64 & 79.85 & 91.27 & 35 & 46.9 & 59.03 & 68.84 \\
\hline Dynamic Pressure [Pa] & 10,000 & 715 & 151 & 101 & 10,000 & 8000 & 4000 & 1900 \\
\hline Velocity $[\mathrm{km} / \mathrm{s}]$ & 1.53 & 3.07 & 4.22 & 5.48 & 1.54 & 3.29 & 4.73 & 5.96 \\
\hline Flight-path angle $\left[^{\circ}\right]$ & -3.31 & -1.54 & -0.38 & -0.55 & -3.32 & -1.54 & -0.38 & -0.55 \\
\hline Angle of Attack [ $\left.{ }^{\circ}\right]$ & 3.77 & 4.31 & 4.39 & 2.96 & 11.82 & 14.08 & 18.12 & 28.76 \\
\hline Sideslip Angle $\left[{ }^{\circ}\right]$ & 0 & 0 & 0 & 0 & 0 & 0 & 0 & 0 \\
\hline Bank Angle $\left[{ }^{\circ}\right]$ & 0 & 0 & 0 & 0 & 57.81 & -59.38 & -29.80 & 59.42 \\
\hline Thrust [kN] & $14.7 \cdot 10^{3}$ & $7.2 \cdot 10^{3}$ & $7.2 \cdot 10^{3}$ & $5 \cdot 10^{3}$ & \multicolumn{4}{|c|}{0} \\
\hline Gross mass [kg] & $510 \cdot 10^{3}$ & $340 \cdot 10^{3}$ & $270 \cdot 10^{3}$ & $180 \cdot 10^{3}$ & \multicolumn{4}{|c|}{$102 \cdot 10^{3}$} \\
\hline$x_{C o M}^{\star}[\mathrm{m}]$ & $(35.6,0,2.8)$ & $(36.6,0,2.8)$ & $(36.7,0,2.8)$ & $(36.8,0,2.8)$ & \multicolumn{4}{|c|}{$(37.7,0,2.8)$} \\
\hline$I_{x x}\left[\mathrm{~kg} \mathrm{~m}^{2}\right]$ & $6646 \cdot 10^{3}$ & $4878 \cdot 10^{3}$ & $4187 \cdot 10^{3}$ & $3320 \cdot 10^{3}$ & \multicolumn{4}{|c|}{$2489 \cdot 10^{3}$} \\
\hline$I_{y y}\left[\mathrm{~kg} \mathrm{~m}^{2}\right]$ & $81,680 \cdot 10^{3}$ & $59,645 \cdot 10^{3}$ & $51,012 \cdot 10^{3}$ & $40,142 \cdot 10^{3}$ & \multicolumn{4}{|c|}{$29,115 \cdot 10^{3}$} \\
\hline$I_{z z}\left[\mathrm{~kg} \mathrm{~m}^{2}\right]$ & $82,351 \cdot 10^{3}$ & $60,013 \cdot 10^{3}$ & $51,262 \cdot 10^{3}$ & $40,244 \cdot 10^{3}$ & \multicolumn{4}{|c|}{$29,123 \cdot 10^{3}$} \\
\hline$I_{x y}\left[\mathrm{~kg} \mathrm{~m}^{2}\right]$ & $-11 \cdot 10^{3}$ & $-13 \cdot 10^{3}$ & $-14 \cdot 10^{3}$ & $-17 \cdot 10^{3}$ & \multicolumn{4}{|c|}{$-50 \cdot 10^{3}$} \\
\hline$I_{x z}\left[\mathrm{~kg} \mathrm{~m}^{2}\right]$ & $1 \cdot 10^{3}$ & $1 \cdot 10^{3}$ & $1 \cdot 10^{3}$ & $0 \cdot 10^{3}$ & \multicolumn{4}{|c|}{$-10 \cdot 10^{3}$} \\
\hline$I_{z y}\left[\mathrm{~kg} \mathrm{~m}^{2}\right]$ & $5377 \cdot 10^{3}$ & $3713 \cdot 10^{3}$ & $3165 \cdot 10^{3}$ & $2417 \cdot 10^{3}$ & \multicolumn{4}{|c|}{$1737 \cdot 10^{3}$} \\
\hline
\end{tabular}

Table 2.6: FSS-1 constraints: maximum system capabilities [Daimler-Benz Aerospace, 1998].

\begin{tabular}{||lc||}
\hline Parameter & Constraint \\
Maximum vehicle acceleration in x-direction $a_{x}\left[\mathrm{~m} / \mathrm{s}^{2}\right]$ & $3.0 \cdot g_{0}$ \\
Maximum vehicle acceleration in z-direction $a_{z}\left[\mathrm{~m} / \mathrm{s}^{2}\right]$ & $0.5 \cdot g_{0}$ \\
Maximum body flap deflection $[\mathrm{deg}]$ & 20 \\
Minimum body flap deflection [deg] & -10 \\
Maximum body flap deflection rate $[\mathrm{deg} / \mathrm{s}]$ & 3 \\
Maximum elevon deflection [deg] & 30 \\
Minimum elevon deflection [deg] & -20 \\
Maximum elevon deflection rate [deg/s] & 40 \\
Maximum rudder deflection [deg] & 30 \\
Minimum rudder deflection [deg] & 0 \\
Maximum rudder deflection rate $[\mathrm{deg} / \mathrm{s}]$ & 40 \\
Maximum gimbal angle [deg] & 4 \\
Minimum gimbal angle [deg] & -4 \\
Maximum gimbal rate [deg/s] & 4 \\
Maximum roll thruster moment $[\mathrm{Nm}]$ & 3500 \\
Maximum pitch thruster moment $[\mathrm{Nm}]$ & 45700 \\
Maximum yaw thruster moment [Nm] & 21400 \\
\hline
\end{tabular}

2.5. It should be noticed no information about the bank angle along the ascent trajectory is offered by DaimlerBenz Aerospace [1998], thus they are assumed to be zero. This is acceptable for the thesis project, since we are more interested in the deviation between the actual and the desired attitude, and how efficiently the system realises the commands to eliminate this deviation. Furthermore, the products of inertia are much smaller than the moments of inertia, thus they can be ignored in the formulation of the equations of motion presented in Chapter 4, and in the rest of the analysis. Also, the FSS-1 maximum capabilities are provided in Table 2.6.

\subsection{Mission and System Requirements}

On the basis of characteristics of the reference vehicle and mission, and the objective of this thesis project, the mission requirements and the system requirements can be derived. 


\section{Mission Requirements}

M-1 The simulation of the attitude motion of the FSS-1 spaceplane shall be performed.

Rationale: objective of the research.

M-2 The analyses that shall be executed are:

1. the stability analysis, which comprises of the eigenmotion analysis, trim analysis, and flying-quality analysis;

2. the controllability analysis;

3. the analysis of the response to atmospheric disturbances.

Rationale: objective of the research.

Specification: the atmospheric perturbations will consist of steady-state wind, turbulence, and wind gust from any direction. Their models and magnitude are discussed in Section 3.3.

M-3 The analyses shall be conducted in the characteristic points with Mach 5, 10, 15, 20 along both the hypersonic ascent and re-entry trajectory.

Rationale: objective of the research.

M-4 The control system shall be designed so that Level-1 of MIL requirements are achieved.

Rationale: functional to achieve the objective of the research. MIL-Specs are presented in Section 5.2.

M-5 The nominal conditions of the design points of the FSS-1 trajectory shall be in compliance with the values used by Daimler-Benz Aerospace [1998].

Rationale: reference vehicle.

Specification: The nominal conditions of the design points of the FSS-1 trajectory are listed in Table 2.5. Note that these values are deduced from graphics. Since they are not very accurate source of data, these values cannot be defined very precisely. However, they should be adequate for our analysis.

\section{System Requirements}

S-1 The dimensions of the reference vehicle shall be in compliance with the values used by Daimler-Benz Aerospace [1998].

Rationale: reference vehicle characteristics.

Specification: the dimensions of the reference vehicle are listed in Table 2.1.

S-2 Mass and inertia properties of the reference vehicle shall be in compliance with the results by DaimlerBenz Aerospace [1998].

Rationale: derived from reference vehicle's characteristics and reference trajectory.

Specification: mass, centre of mass, and inertia moments and products at the design points are presented in Table 2.5.

S-3 Rocket engines shall be used for propulsion during the ascent flight.

Rationale: reference vehicle's propulsion system.

S-4 The vehicle shall be trimmed by means of body-flap deflection and TVC. If they do not suffice, they shall be supported by elevon deflection.

Rationale: reference vehicle's actuators.

Specification: in Section 5.4.2, the effects of using $100 \%$ body flap, $100 \%$ TVC, or a combination of them to trim the vehicle are studied.

S-5 The vehicle shall be controlled through aerodynamic control (two elevons and two rudders, and a body flap) and propulsion control (TVC, if ascent, and RCS, if re-entry).

Rationale: reference vehicle's actuators.

S-6 The control system shall consist of attitude-angle control (angle of attack, sideslip angle, and bank angle).

Rationale: functional to achieve the objective of the mission. 


\section{3}

\section{Environment}

The environment in which a spaceplane operates affects its trajectory and, consequently, its flying and controllability characteristics. The flight environment generates forces and moments acting on the vehicle that are mainly of gravitational, aerodynamic and propulsive origin. In this chapter an overview of the flight environment is provided. A presentation of the shape and angular motion of the Earth is given in Section 3.1, where also details about its gravity field are provided. Section 3.2 describes the atmospheric models and Section 3.3 defines the wind models. The description presented in this chapter is based on the definitions given by Mooij [2015], if not otherwise specified.

\subsection{Earth's Shape and Gravitational Field}

For flight simulation and flying-quality and controllability analyses purposes, considering all the phenomena that define the shape of the Earth, e.g, Luni-Solar precession and gravitational tides, earthquakes, plate motion, are second-order effects when compared with, e.g., an ellipsoid Earth model, and require longer CPU time. Thus, they are neglected since they are beyond the level of accuracy required by this analysis.

For flight simulation it seems an adequate approximation to consider the Earth as an ellipsoid of revolution characterized by ellipticity $e$, mean equatorial radius $R_{e}$ larger than mean polar radius $R_{p}$, and rotating with constant angular velocity $\omega_{\mathbf{R}}=\left(0,0, \omega_{c b}\right)^{T}$. The ellipticity is computed as:

$$
e=1-\frac{R_{p}}{R_{e}}
$$

which can be used to derive the radius at an arbitrary point along the surface. Since it is possible to assume, to a first approximation, that the geographic latitude and geodetic latitude are the same, the expression of the radius at an arbitrary point along the surface is:

$$
R_{s}=R_{e}\left(1-e \sin ^{2} \delta\right)
$$

with $\delta$ being the geographic latitude. Thus, the altitude can be computed as: $h=R-R_{e}\left(1-e \sin ^{2} \delta\right)$.

To study the flying qualities and the controllability characteristics of a spaceplane at certain interesting points along its trajectory, the oblateness and the rotation of the Earth have a second-order effect with respect to the approximation of a spherical non-rotating Earth. Consequently, the latter approximation is considered to be the optimal choice for our needs. In this case the Earth's angular velocity is zero, $\omega_{c b}=0$, and its radius is constant and equal to the equatorial radius, $R_{e}$. The altitude of a body above the surface can simply be calculated as follows:

$$
h=R-R_{e}
$$

The shape and mass distribution of the Earth strongly influence its gravitational field. Assuming the Earth and the vehicle as points of mass, the gravitational force applied to the vehicle by the Earth is simply defined by the Newton's law of gravitation:

$$
\mathbf{F}_{\mathbf{g}}=\frac{G M m}{R^{2}} \hat{\mathbf{R}}=\frac{\mu m}{R^{2}} \hat{\mathbf{R}}
$$


where $\hat{\mathbf{R}}$ is the normalised position vector, $\mathrm{R}$ is the distance between the two bodies, $\mathrm{M}$ is the central body's mass, $\mathrm{m}$ the vehicle's mass, and $\mu$ is the gravitational constant, which depends on the mass of the planet considered since it is defined as $\mu=G M$ with $\mathrm{G}$ being the universal gravity constant $\left(G=6.668 \cdot 10^{11} \mathrm{~m}^{3} / \mathrm{kg} \mathrm{s}^{2}\right)$. For the Earth $\mu$ is equal to $3.9860047 \cdot 10^{14} \mathrm{~m}^{3} / \mathrm{s}^{2}$ [Wakker, 2015]. The gravitational potential can be expressed as:

$$
U=-\frac{\mu}{R}
$$

from which the definition of gravitational force can be defined:

$$
\mathbf{F}_{\mathbf{g}}=-m \nabla U
$$

with $\nabla$ as the gradient operator, $\nabla=\frac{\partial}{\partial R}$.

This approximation is known as central field model. It indicates that the magnitude of the gravitational field decreases quadratically with the radial distance. Considering that we aim to study the characteristics of the spaceplane rotational motion at certain points along the trajectory, the altitude remains constant during the whole simulation. Moreover, the variation of latitude and longitude are not interesting for our analysis. We can conclude that gravity is constant at each point of the trajectory and, applying the Newton's gravitational law, equal to:

$$
g=\frac{g_{0} R_{0}^{2}}{\left(R_{0}+h\right)^{2}}
$$

where $\mathrm{g}_{0}$ is the gravitational acceleration at the Earth's surface $\left(\mathrm{g}_{0}=9.80665 \mathrm{~m} / \mathrm{s}^{2}\right), \mathrm{R}_{0}$ is the radial distance at which $g=g_{0}$, i.e., $R_{0}=6378.137 \mathrm{~km}$ [Wakker, 2015].

\subsection{Atmosphere}

Earth's atmosphere is responsible for the generation of two of the most important forces that act and, consequently, influence on the vehicle during flight, i.e., lift and drag. From the main features of the atmospheric model, such as density, temperature, and the dynamic viscosity of the flow, other important characteristics of flight can be computed, such as Mach and Reynolds number, and speed of sound. Even if the atmosphere is characterized by several dynamic processes that are very complicated to model, it has been shown that the satisfactory simulation are obtained also when considering averaging atmospheric parameters.

Several model of the Earth's atmosphere exist. An approximate model of the Earth's atmosphere is the socalled Exponential Atmospheric Model, which considers the atmosphere as composed by an ideal gas at constant temperature in a hydrostatic equilibrium. This atmospheric model is easy to implement and generally used analytical approach, but it is too simplified and it can not ensure an adequate accuracy for flight simulation and stability analysis. However, the United States Standard Atmosphere 1976 (US76) [NOAA et al., 1976] model seems to be advantageous for our application as it is a straightforward model, easy to verify and used for many engineering applications. For geopotential altitude The US76 below $86 \mathrm{~km}$ considers the atmosphere molecular mass $M$ constant and equal to its value at sea level, i.e., $M_{0}=28.9644 \mathrm{~kg} / \mathrm{kmole}$, and that the temperature is equal to the molecular scale temperature $\mathrm{T}_{M}$ that is defined as:

$$
T_{M}=T_{M i}+L_{z i}\left(z-z_{i}\right)
$$

where $i$ indicates the $i$-th layer, considering the total altitude range divided into layers, $L$ is the scale height equal to $L=\frac{R T}{g_{0}}$ with $\mathrm{R}$ being the air gas constant $(R=287 \mathrm{~J} / \mathrm{kg} \mathrm{K})$, and $z$ the geopotential altitude. Figure 3.1 illustrates the temperature profile as function of altitude comparing the US76 model and the exponential atmosphere model. For $h \leq 86 \mathrm{~km}$, the temperature is $T=T_{M}$. Differently, for $86 \leq h \leq 91 \mathrm{~km}$, the temperature is constant and equal to $T=186.8673 \mathrm{~K}$. The upper layers of the atmosphere are not interesting for this thesis project. Once $T$ is known, the atmospheric pressure can be determined as following: :

$$
p=\frac{N R^{\star} T}{N_{A}}
$$

where $N$ is the total number density, tabulated by NOAA et al. [1976], $N_{A}=6.0225 \cdot 10^{26} \mathrm{kmol}^{-1}$ is the Avogadro's constant, and $R^{\star}=8314.32 \mathrm{~J} / \mathrm{kmoleK}$ the universal gas constant. At this point, the density $\rho$ can be calculating by inversion of the ideal gas law, which relates pressure $p$ with density $\rho$ and temperature $T$ by means of the proportionality constant $R$ as follows:

$$
p=\rho R T
$$




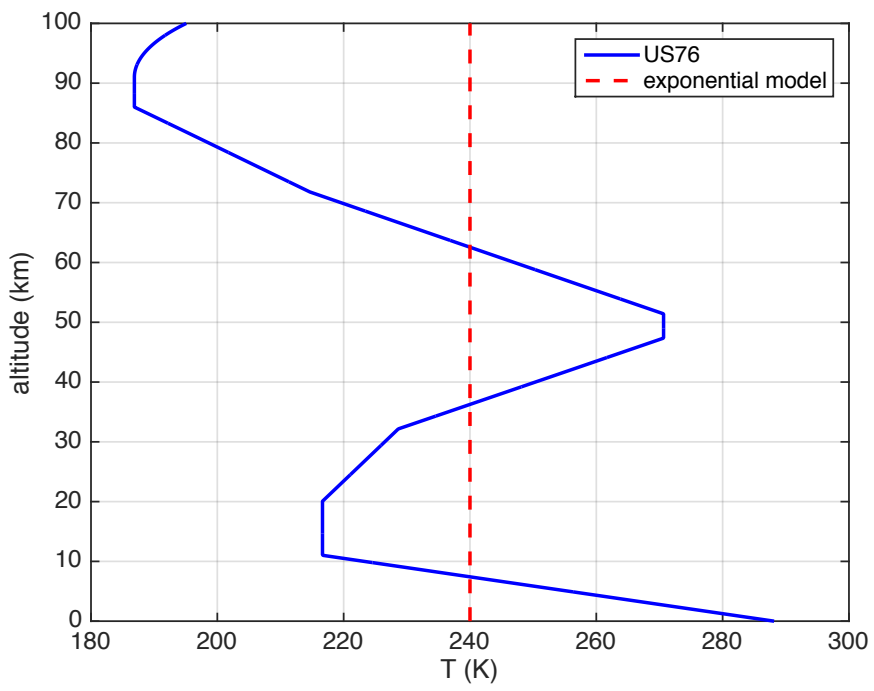

Figure 3.1: US Standard Atmosphere 1976 and Exponential Atmosphere Model: Temperature Profile. [Mooij, 2015]

The Mach number is defined as the ratio between relative airspeed and speed of sound:

$$
M=\frac{V_{a}}{a}
$$

where $a$ is the speed of sound defined as $a=\sqrt{\gamma R T}$, with $\gamma$ being the ratio between the specific heat at constant pressure and specific heat at constant volume and, for the range of altitude considered, it can be considered constant and equal to $\gamma=1.4$.

\subsection{Wind Model}

Wind is a current of air blowing in a specified direction due to differences of pressure in the atmosphere. It has a stochastic nature, thus a specific wind field cannot be assumed and predetermined. It acts as a disturbance for the motion of a vehicle. Studying how a spaceplane reacts to wind disturbances could be essential to determine its flying qualities and controllability characteristics.

Wind can come from any direction and have a different magnitude depending on location and time. As a consequence, modelling wind could be very complicated. For the simulation of an aerospace flight in a particular wind condition, wind is generally modelled analysing the mean effect and the relative standard deviation. Generally, it is convenient to identify different components of the wind vector and model each component individually. The most common components are the horizontal steady state wind, turbulence, and horizontal and vertical gusts, which are treated in the following sections.

\subsubsection{Steady-State Wind}

Wind profiles can be modelled in different ways. For example, when we are interested in studying a specific flight phase, it is possible to consider a constant wind irrespective of the vehicle's location in the atmosphere. Another option is to model the wind profile as a function of altitude. A wind model, which is constant or altitude dependent, is quite easy to define and it requires little computational time, but a more realistic representation of the wind is provided by the horizontal wind model (HWM07), released in 2007 [Drob et al., 2008]. It models atmospheric wind as a global horizontal steady-state wind. It requires the implementation of a larger number of equations than the other, simpler models, but it results in more accurate results and includes a time and location dependency. The description of the HWM07 model presented in this section is based on the work by Drob et al. [2008]. Here, the wind velocity vector is defined as:

$$
\mathbf{U}(t, \tau, \delta, \theta)=\sum_{i} \gamma_{i} \mathbf{u}_{i}(t, \tau, \delta, \theta)
$$

with $t$ being the time, $\tau$ the geodetic longitude, $\delta$ the geodetic latitude, $\theta$ the local solar time, $\gamma_{i}$ the weighting terms and $\mathbf{u}_{i}$ can be the zonal wind component $u$ or the meridional wind component $v$. 



Figure 3.2: Zonal and meridianal maps computed with the HWM07 (April 1, at 12:00). ${ }^{1}$

The zonal wind component $u$ is calculated as follows:

$$
\begin{aligned}
u(t, \tau, \delta, \theta) & =\sum_{n=0}^{N} \sum_{s=0}^{S}\left\{C_{r}^{n, s} \Psi_{1}^{n, s}-C_{i}^{n, s} \Psi_{2}^{n, s}+B_{r}^{n, s} \Psi_{3}^{n, s}+B_{i}^{n, s} \Psi_{4}^{n, s}\right\}+ \\
& +\sum_{l=1}^{L} \sum_{n=l}^{N} \sum_{s=0}^{S}\left\{C_{r}^{l, n, s} \Psi_{1}^{l, n, s}-C_{i}^{l, n, s} \Psi_{2}^{l, n, s}+B_{r}^{l, n, s} \Psi_{3}^{l, n, s}+B_{i}^{l, n, s} \Psi_{4}^{l, n, s}\right\}+ \\
& +\sum_{m=1}^{M} \sum_{n=m}^{N} \sum_{s=0}^{S}\left\{C_{r}^{m, n, s} \Psi_{1}^{m, n, s}-C_{i}^{m, n, s} \Psi_{2}^{m, n, s}+B_{r}^{m, n, s} \Psi_{3}^{m, n, s}+B_{i}^{m, n, s} \Psi_{4}^{m, n, s}\right\}
\end{aligned}
$$

where $C_{r}, C_{i}, B_{r}$, and $B_{i}$ are the spectral coefficients with s, $1, \mathrm{~m}, \mathrm{n}$ being the seasonal, local time, longitudinal and latitudinal spectral wave numbers. These spectral coefficients are estimated experimentally for maximum values $\mathrm{S}=2, \mathrm{~L}=3, \mathrm{M}=2$, and $\mathrm{N}=8$. Moreover, $\Psi_{1}, \Psi_{2}, \Psi_{3}$, and $\Psi_{4}$ are the modulated basis functions of the Fourier series that are calculated with the following expressions:

$$
\begin{aligned}
& \Psi_{1}^{g, n, s}=-\frac{\cos g \beta}{\sqrt{n(n+1)}} \frac{d \bar{P}_{n}^{g}(\phi)}{d \phi}[\cos (s t)+\sin (s t)] \\
& \Psi_{2}^{g, n, s}=\frac{\sin g \beta}{\sqrt{n(n+1)}} \frac{d \bar{P}_{n}^{g}(\phi)}{d \phi}[\cos (s t)+\sin (s t)] \\
& \Psi_{3}^{g, n, s}=-\frac{\sin g \beta}{\sqrt{n(n+1)}} \frac{\bar{P}_{n}^{g}(\phi)}{\cos \phi}[\cos (s t)+\sin (s t)] \\
& \Psi_{4}^{g, n, s}=-\frac{\cos g \beta}{\sqrt{n(n+1)}} \frac{\bar{P}_{n}^{g}(\phi)}{\cos \phi}[\cos (s t)+\sin (s t)]
\end{aligned}
$$

The meridional wind component $v$ is determined in an analogous way, applying the parity relationship, which is $u:\left\{C_{r}, C_{i}, B_{r}, B_{i}\right\} \longrightarrow v:\left\{B_{r}, B_{i},-C_{r},-C_{i}\right\}$, to Equation (3.13). In the preceding equations the term $\bar{P}_{n}^{g}(\phi)$ indicates the n-th Legendre polynomial, while $d \bar{P}_{n}^{g}(\phi)$ indicates the derivative of the n-th Legendre polynomial. The HWM07 model is available in Matlab-Simulink® , which is the modelling environment of choice (see Chapter B). Figure 3.2 presents the zonal and meridianal maps computed with the HWM07.

\subsubsection{Wind Gusts}

Wind gusts are perturbations to which a spaceplane can be subjected during its flight through the atmosphere. Wind gusts can be considered to be a variation of wind magnitude and/or direction. There are mainly two types of wind gust models, i.e., a spectral model and a discrete model. The former is determined through a random process, while the latter defines a single gust with specified magnitude and shape. The spectral model has a very complex form and it is difficult to implement, while the discrete model is much simpler and is still adequate for engineering purposes. So, the discrete wind gust model is chosen to be used in our thesis work.

A wind gust discrete model is the so-called 1-cosine model [NASA, 2000]. Let $x$ be the horizontal distance and $x=0$ the origin of the gust, then the 1 -cosine model defines that the velocity of the gust is zero for $x<0$ and

${ }^{1}$ Erwin Mooij, private communication (Sept 2017). 


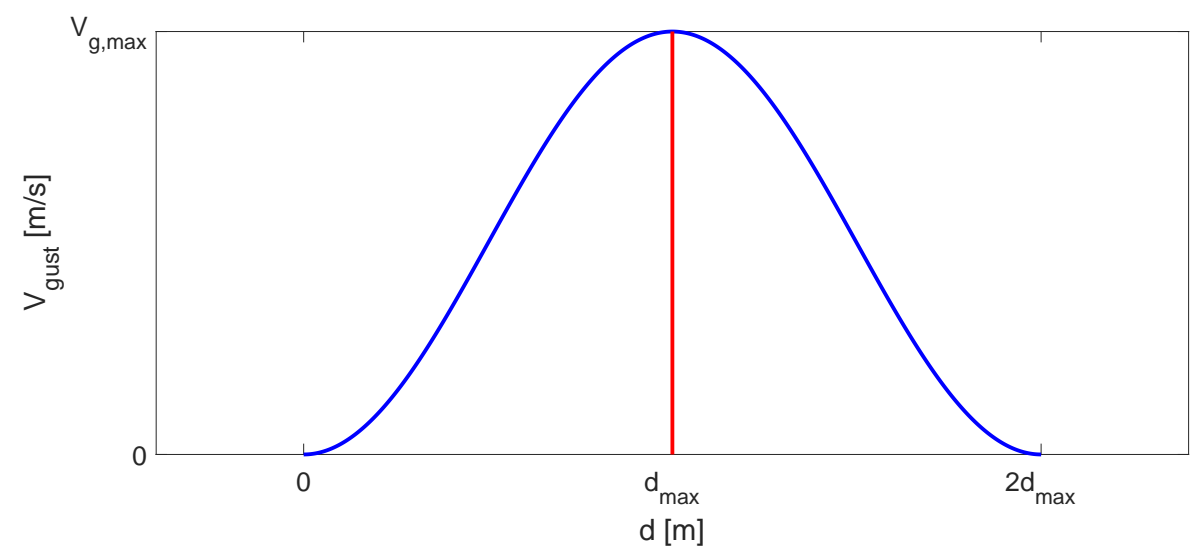

Figure 3.3: The 1-cosine discrete gust model.

for $x>2 d_{\text {max }}$, so the gust can affect the motion of any body that is located at $0<x<2 d_{\text {max }}$. The term $d_{\text {max }}$ is used to indicate the distance called gust half-width at which the velocity of the gust is maximum $V_{g}=V_{g, \text { max }}$, as illustrated in Figure 3.3. In particular, it specifies that:

$$
V_{g}= \begin{cases}0 & \text { if } x<0 \\ \frac{V_{g, \text { max }}}{2}\left[1-\cos \left(\frac{\pi x}{d_{\text {max }}}\right)\right] & \text { if } 0<x<2 d_{\text {max }} \\ 0 & \text { if } x>2 d_{\text {max }}\end{cases}
$$

To calculate $\mathrm{V}_{g, \max }$, NASA [2000] provides curves that define the relationship between $\frac{V_{g, \max }}{\sigma}$ and $\frac{d_{\max }}{L}$. These curves are derived on the basis of the Dryden turbulence spectrum for a single probability of exceeding the gust magnitude. Also the 1-cosine model is available in Matlab-Simulink®.

\subsubsection{Turbulence}

It is interesting to examine how the system reacts to a perturbation such as turbulence, whether the perturbation destabilises the system ot it is controllable, and in the latter case how much time is needed to come back to the equilibrium condition. The two most commonly used turbulence models are the Von Karman and the Dryden model, which are detailed in MIL-F-8785C [1980] and MIL-HDBK-1797 [1997]. Beal [1993] asserts that, for engineering purposes, the Dryden model is the most commonly used, since it is convenient due to its straightforward form and easy way of passing white noise through a linear filter with respect to other models, such as the Von Karman model. Therefore, the Dryden model will be used and detailed in this section. Again, this model is available in Matlab-Simulink ${ }^{\circledR}$.

The Dryden model introduces turbulence to the system by passing band-limited white noise through appropriate forming filters. Turbulence is a stochastic process, which is defined through velocity spectra. The spectra for each of the three turbulence velocities aligned with three main axes of the body-fixed coordinate system are:

$$
\begin{gathered}
\Phi_{u}(\Omega)=\sigma_{u}^{2} \frac{2 L_{u}}{\pi} \frac{1}{1+\left(L_{u} \Omega\right)^{2}} \\
\Phi_{v}(\Omega)=\sigma_{v}^{2} \frac{L_{v}}{\pi} \frac{1+3\left(L_{v} \Omega\right)^{2}}{\left(1+\left(L_{v} \Omega\right)^{2}\right)^{2}} \\
\Phi_{w}(\Omega)=\sigma_{w}^{2} \frac{L_{w}}{\pi} \frac{1+3\left(L_{w} \Omega\right)^{2}}{\left(1+\left(L_{w} \Omega\right)^{2}\right)^{2}}
\end{gathered}
$$

with $\Phi_{u}, \Phi_{v}$, and $\Phi_{w}$ being the Power Spectral Densities (PSD) $\left(\mathrm{m}^{3} / \mathrm{s}^{2}\right)$ of the velocity components along the $x$-, $y$-, and $z$-axes of the vehicle, that are respectively $u, v$, and $w(\mathrm{~m} / \mathrm{s})$. The standard deviations are indicated with $\sigma_{u}, \sigma_{v}$, and $\sigma_{w}(\mathrm{~m} / \mathrm{s})$, the turbulence scale lengths with $L_{u}, L_{v}$, and $L_{w}(\mathrm{~m})$, and the spatial frequency with $\Omega(\mathrm{m} / \mathrm{s})$. The turbulence scale length $L$ and turbulence intensity $\sigma$ are the parameters of the Dryden model. Indeed, their values determine the shape of the PSD and the quality of the model, which is higher when the spectra of the model fit better the observed turbulence spectra. In the MIL-Specs the scale length $L$ and turbulence intensity $\sigma$ are specified based on the data obtained through turbulence observation. For 


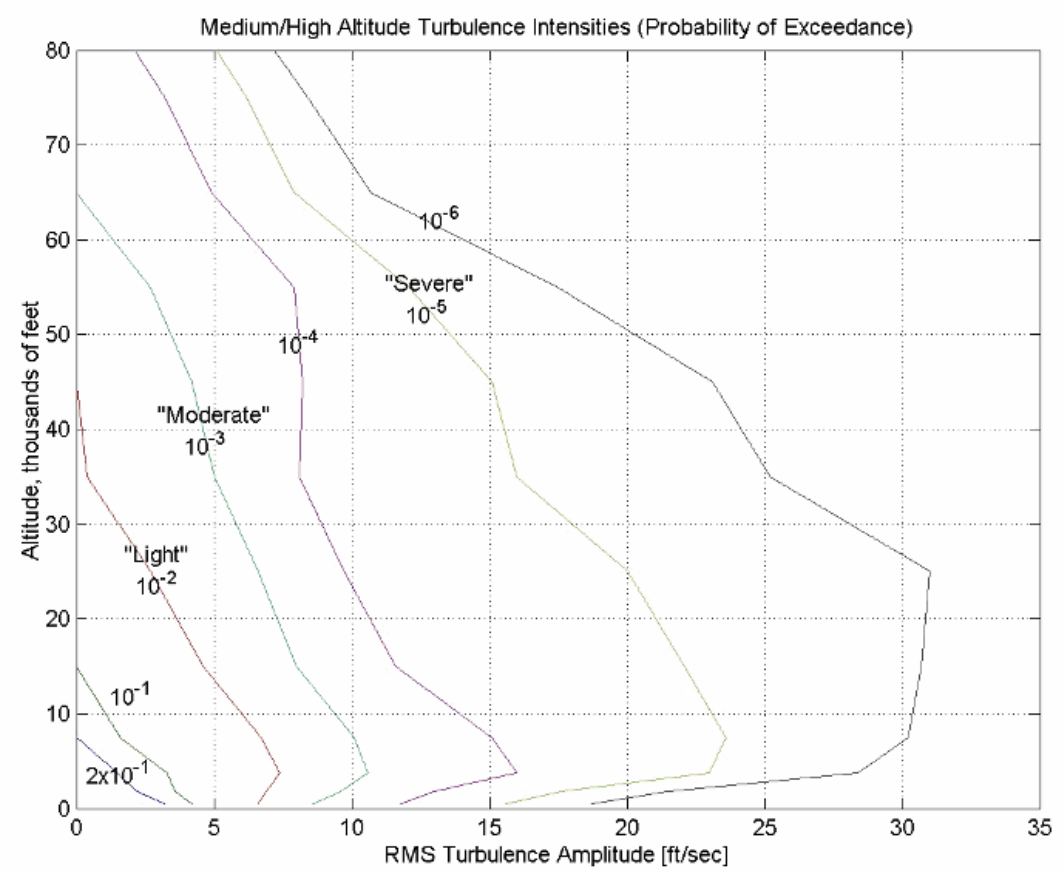

Figure 3.4: Turbulence intensities[MIL-F-8785C, 1980].

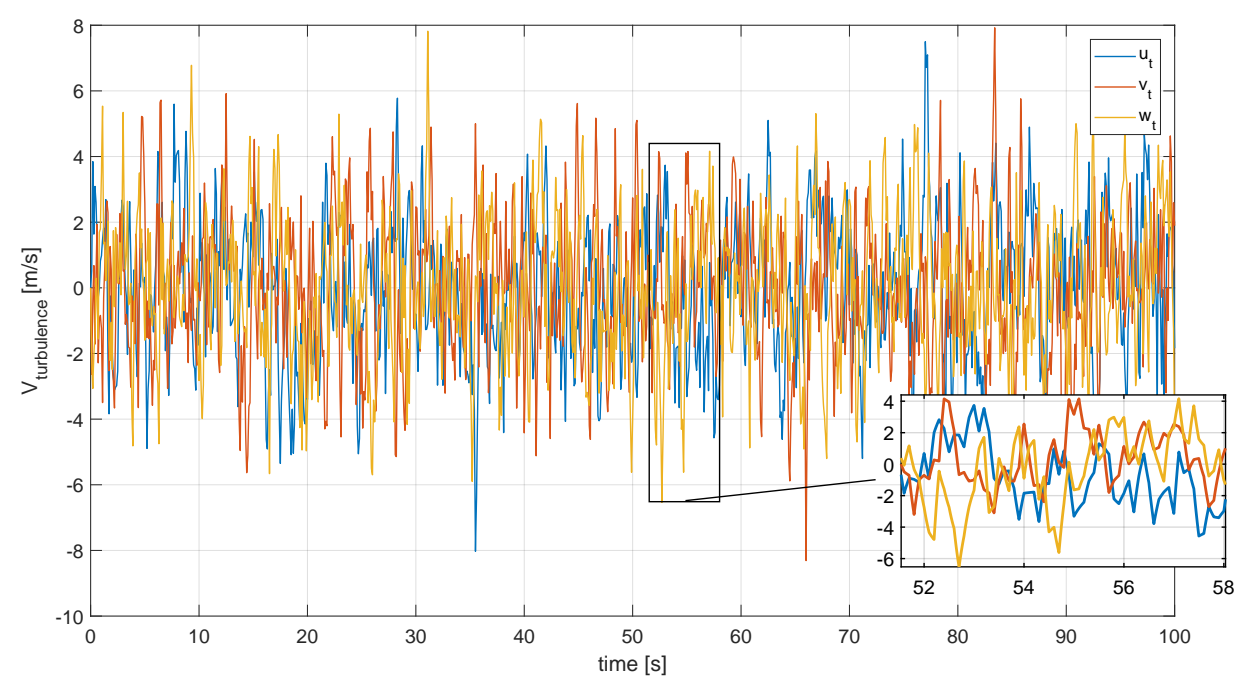

Figure 3.5: Example of severe turbulence components at the design point with Mach 5 along re-entry.

the region of altitudes that interests this thesis project $(h \geq 600 \mathrm{~m})$, the turbulence scale lengths are defined as [MIL-HDBK-1797, 1997]:

$$
L_{u}=L_{v}=L_{w}=533.4 \mathrm{~m}(1750 \mathrm{ft})
$$

The turbulence intensities are determined by means of Figure 3.4 that gives the root-mean-square (RMS) of the turbulence intensities as function of altitude and probability of exceeding the turbulence intensity. The relation between the turbulence intensities is $\sigma_{u}=\sigma_{\nu}=\sigma_{w}$. The Dryden model is available in Matlab-Simulink®. To conclude the description of turbulence, Figure 3.5 shows the variation of the turbulence components in time at the design point with Mach 5 along the re-entry. 


\section{Flight Mechanics}

The motion of a body under the effects of external forces and torques of various kinds constitutes one of the most fundamental problem in physics. An example of such problem is the flight of an spaceplane inside/outside a planetary atmosphere. In this case, the vehicle is the hypersonic spaceplane FSS-1 and the external forces and torques acting on it are generated by the gravitational field of the Earth, the thrust produced by its engines, and the aerodynamic forces and moments due to the passage of the vehicle through air. The control of the vehicle is directly linked to the motion of the spaceplane. Indeed, the goal of the control system is to utilise the dynamics capabilities of the system to realise the control commands and achieve the desired attitude.

The mechanics are based on the well-known Newton's laws of motion and law of gravitation, from which the equations of motion (EoM) of a vehicle can be derived. To completely determine the motion, it is important to define the reference frames and the state variables, on which the description of the motion is dependent. Moreover, since sometimes it is convenient to define forces in different reference frames, it is required to transform one frame to another, and viceversa.

Given the several analyses to be performed to achieve the thesis objectives, different models are needed so that the proper results can be generated. To clarify what models are used and link them with the simulation and analyses that will be performed, a blueprint of the dynamic models is presented in Section 4.1. Section 4.2 identifies the reference frames, Section 4.3 introduces the state variables, and Section 4.4 presents the transformations from one frame to another. The external forces and moments, which need to be implemented in the EoM since they are acting on the vehicle and influencing its motion are determined in Section 4.5. At this point, it is possible to fully define the equations of motion in Section 4.6. Here, the non-linear translational and rotational EoM are presented, followed by the description of how the presence of wind needs to be taken into account in the EoM. Finally, Section 4.7 provides the linearised set of equations of motion written in state-space form, which enable the analysis of the stability of the system.

\subsection{Dynamic Models Blueprint}

The purpose of this thesis work is twofold. We desire to simulate the motion of a hypersonic spaceplane and analyse its flying qualities and controllability characteristics. To perform the simulations, the set of EoM needs to be as accurate as possible. Thus, the non-linear set of equations of motion is appropriate for this scope with the assumptions of oblate Earth that rotates with a constant angular velocity $\boldsymbol{\omega}_{\boldsymbol{R}}=\left(0,0, \omega_{c b}\right)^{T}$. For the second objective, it is convenient and common practise to linearise the equations of motion. Indeed, linearised EoM allow to present the time derivatives of the states as a linear combination of the states so that the eigenvalues and eigenvectors that describe the characteristic motion can be calculated. In this case, the Earth's oblateness and rotation have a second-order effect, thus a spherical non-rotating Earth can be assumed.

Moreover, mission requirement $M-2$ defines that the analyses that need to be conducted to achieve the thesis goal are multiple. This variety requires the use of more than one dynamic model to generate the relevant results. For what concerns the non-linear models, two models are required:

1. a model defined by the translational and rotational EoM for 6-DoF simulations

2. a model defined by the rotational EoM for 3-DoF simulations 


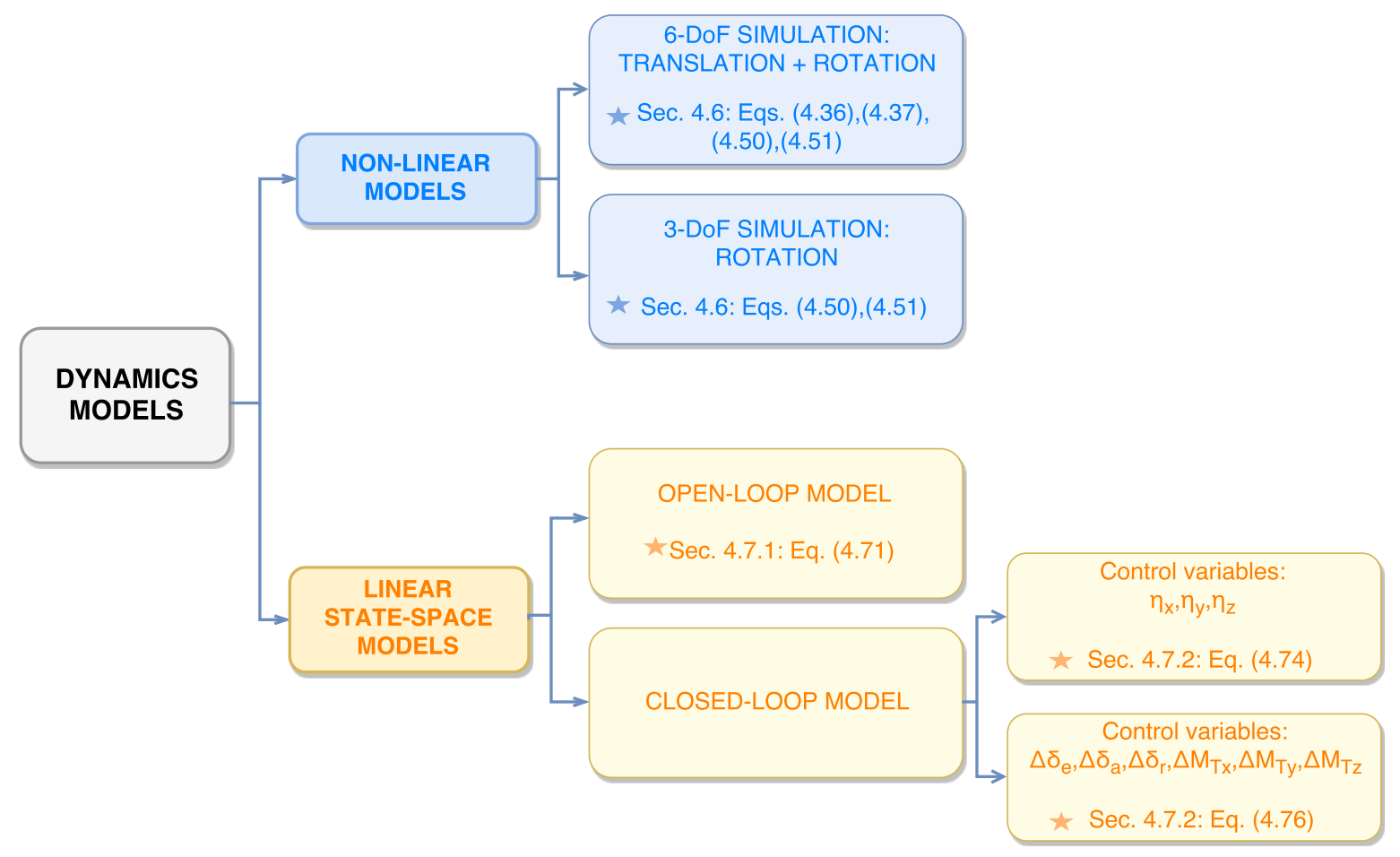

Figure 4.1: Blueprint of the dynamic models used in this thesis work.

where the first one is required to verify that the corrected HORUS aerodynamic database is adequate to describe the FSS-1 behaviour during flight, as done in Section 2.1.2, and the second model is needed to simulate the rotational motion of the vehicle when studying the response to control commands or disturbances.

For control design and for the study of the stability and controllability properties, the linearised set of EoM is generally written in matrix form, which is known as the state-space form. It facilitates the identification and examination of the characteristics of motion. Different state-space models are needed for the analyses:

1. a model defined by translational and rotational EoM

2. a model defined by rotational EoM with moment-fractions as control variables

3. a model defined by rotational EoM, with control-surface deflections and thruster moments as control variables

The first model is required to conduct the stability analysis, which comprises of the study of the eigenmotion, trimmability, and flying qualities. Indeed, all the state variables describing both translational and rotational motion need to be characterised to identify the modes of motion. For the stability analysis, the vehicle is in open-loop, i.e., no control is applied to the system. This means that the deviations of the control variables are set to zero. This model is identified as the open-loop model. A state-space model of the linearised set of rotational EoM is necessary to design the control system. Indeed, the other two models intend to describe the relation between the state deviations and the induced corrective control. Thus, they will be indicated as closed-loop models. In particular, to design the control system for our reference vehicle, the moment fractions are used as control variables, with the moment fractions being the commanded fraction of the maximum available control moments. Also, to verify the control system, it is useful to implement a model similar to the one used in some previous work where the attitude controller for HORUS was designed [Brinkman, 2017][Rijnsdorp, 2017] [Mooij, 1998a], so that an acceptance test can be performed. This model has as control variables the control-surface deflections and the thruster moments.

On the basis of what is discussed before, Figure 4.1 illustrates the blueprint of the singular dynamics models that are needed to achieve the objectives. For each model the section where this model is defined is specified, together with the equations that define the model. In the following, the characteristics of these models are identified, which comprehend the used state variables, the reference frames where they are defined, and the equations that describe the vehicle's motion. 


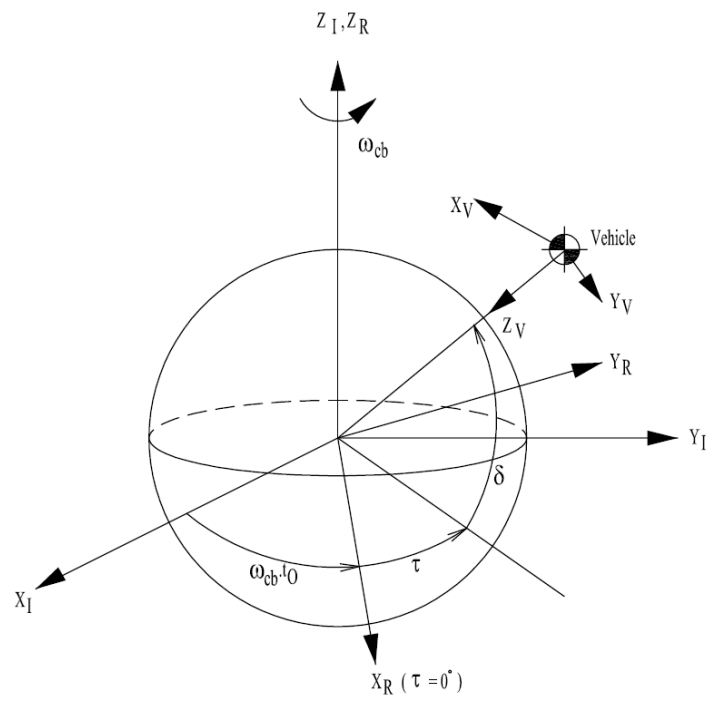

Figure 4.2: Graphical representation of the relationship between $I-, R$ - and $V$-frames [Mooij, 2015].

\subsection{Reference Frames}

A reference frame is a key element to determine the motion of a body unambiguously. A reference frame is represented by three mutually orthogonal axes, which intersect at a point called origin and whose direction describes the orientation of the frame. The motion of a body is defined by mathematical variables, known as coordinate systems, that are evaluated with respect to a specific reference frame. In other words, a reference frame defines the space in which the motion of a body is described.

We can distinguish between inertial and non-inertial reference frames. Bodies in inertial frames, whose total force acting upon them is zero, are at rest or in rectilinear motion with constant velocity. The physical laws have the same form in all the inertial frame of reference. A non-inertial reference frame is accelerating with respect to an inertial frame, thus, when the motion is described with respect to a non-inertial reference frame, we need to consider additional forces, that are called fictitious or apparent forces. For examples, for rotating reference frames, the Coriolis and centrifugal forces have to be added to the equations of motion.

Since certain forces are defined in specific reference frames for the sake of convenience, several reference frames are needed to derive the equations of the motion of a spaceplane. In the following, the reference frames that are useful for our analysis are presented. Note that all the frames of reference presented are right-handed and defined by three mutually orthogonal unit vectors. Moreover, the vehicle is assumed to be geometrically symmetric at least along one plane (i.e., in longitudinal direction). Two types of reference frames are treated: Earth-centred frames and vehicle fixed frames. The description of the reference frames, indicated with $\mathscr{F}$ in the following of this report, is based on the standard definitions given by Mooij [1994].

\section{Inertial Earth-Centred Reference Frame $\left(\mathscr{F}_{I}\right)$}

The inertial Earth-centred reference frame is identified with index $I$. It is centred at the CoM of the Earth. The $\mathrm{X}_{I} \mathrm{Y}_{I}$-plane coincides with the Earth equatorial plane with the $\mathrm{X}_{I}$-axis pointing at the vernal equinox, which is a point in the star sign Aries, at 12 o'clock on 1 January 2000. The $\mathrm{Z}_{I}$-axis is pointing north, coinciding with the Earth rotational axis, and the $\mathrm{Y}_{I}$-axis completes the right-handed system.

\section{Rotating Earth-Centred Reference Frame $\left(\mathscr{F}_{R}\right)$}

The rotating Earth-centred reference frame is identified with index $R$. It is centred at the CoM of the Earth and it rotates together with the Earth. The $\mathrm{Z}_{R}$-axis is pointing north, the $\mathrm{X}_{R}$-axis intersects the equator at zero longitude and the $\mathrm{Y}_{R}$-axis completes the right-handed system.

\section{Vertical Reference Frame $\left(\mathscr{F}_{V}\right)$}

The vertical reference frame is identified with index $V$. Its origin is located at the CoM of the vehicle. The $\mathrm{Z}_{V^{-}}$ axis is directed along the radial component of the gravitational acceleration pointing towards the Earth's CoM. The $\mathrm{X}_{V}$-axis lies in a meridian plane pointing to the northern hemisphere. The $\mathrm{Y}_{V}$-axis completes the righthanded system. The $\mathrm{X}_{V} \mathrm{Y}_{V}$-plane is called local horizontal plane. Figure 4.2 illustrates the relation between the $\mathscr{F}_{I}, \mathscr{F}_{R}$ and $\mathscr{F}_{V}$. 


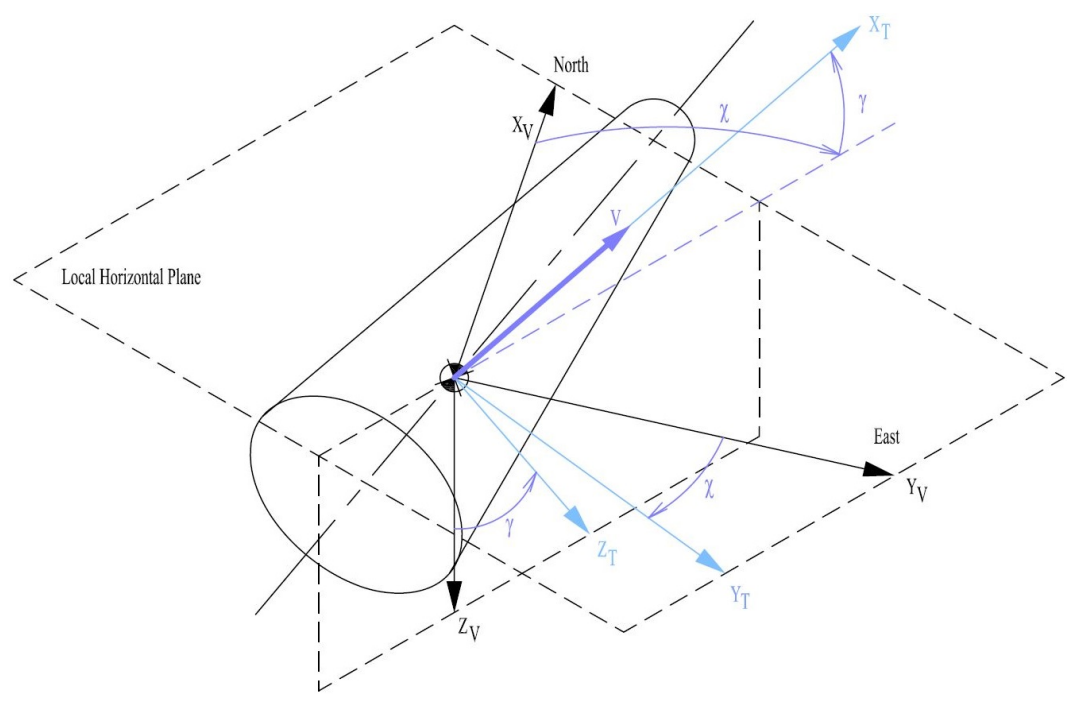

Figure 4.3: Graphical representation of the relationship between $\mathscr{F}_{T A}$ and $\mathscr{F}_{V}{ }^{1}$

\section{Body Reference Frame $\left(\mathscr{F}_{B}\right)$}

The body reference frame is identified with index $B$. It is fixed with the vehicle with the origin located at its CoM. The $\mathrm{X}_{B}$ - and $\mathrm{Z}_{B}$-axis define in the vehicle's plane of symmetry. The former is positive in forward direction and the latter is positive in downward direction. The $\mathrm{Y}_{B}$-axis completes the right-handed system.

\section{Trajectory Reference Frame $\left(\mathscr{F}_{T}\right)$}

The origin of the trajectory reference frame is located at the CoM of the vehicle. It is identified with index $T G$ or $T A$ depending on whether it is groundspeed-based or airspeed-based. We need to define both since we consider wind in our analysis.

The $\mathscr{F}_{T G}$ has the $\mathrm{X}_{T G^{-}}$axis positive in the direction of the velocity vector relative to groundspeed, the $\mathrm{Z}_{T G^{-}}$ axis lies in the vertical plane, pointing downwards, and the $\mathrm{Y}_{T G}$-axis completes right-handed system.

The $\mathscr{F}_{T A}$ has the $\mathrm{X}_{T A^{\text {-axis }}}$ positive in the direction of the velocity vector relative to atmosphere, the $\mathrm{Z}_{T A^{-}}$ axis lies in the vertical plane, pointing downwards, and the $\mathrm{Y}_{T A}$-axis completes right-handed system. Figure 4.3 describes the relationship between $\mathscr{F}_{T A}$ and $\mathscr{F}_{V}$.

\section{Aerodynamic Reference Frame $\left(\mathscr{F}_{A}\right)$}

The origin of the aerodynamic reference frame is located at the CoM of the vehicle. It is identified with index $A G$ or $A A$ depending on whether it is groundspeed-based or airspeed-based. As well as for trajectory frames, both aerodynamic frames are needed in our analysis since we consider wind.

In the $\mathscr{F}_{A G}$ the $\mathrm{X}_{A G}$-axis is positive in the direction of the velocity vector relative to groundspeed, the $\mathrm{Z}_{A G^{-}}$ axis is collinear with the aerodynamic lift force, but with opposite direction, and the $\mathrm{Y}_{A G}$-axis completes righthanded system. This means that $\mathscr{F}_{A G}$ and $\mathscr{F}_{T G}$ coincide when the vehicle is not banking.

In the $\mathscr{F}_{A A}$ the $\mathrm{X}_{A A^{-}}$-axis is positive in the direction of the velocity vector relative to atmosphere, the $\mathrm{Z}_{A G^{-}}$ axis is collinear with the aerodynamic lift force, but with opposite direction, and the $\mathrm{Y}_{A G}$-axis completes righthanded system. Again, $\mathscr{F}_{A A}$ and $\mathscr{F}_{A G}$ coincide when the vehicle is not banking. Figure 4.4 describes the relationship between $\mathscr{F}_{B}, \mathscr{F}_{A}$ and $\mathscr{F}_{T}$.

\section{Wind Reference Frame $\left(\mathscr{F}_{W}\right)$}

The wind reference frame is identified with index $W$. Its origin is located at the vehicle's CoM. The $\mathrm{X}_{W}$-axis has opposite direction of the wind-velocity vector. So, for a northern wind, the $\mathrm{X}_{W}$-axis is positive pointing to north, while, for a wind in the local horizontal plane, the $Z_{W}$-axis is positive in downward direction. The $\mathrm{Y}_{W}$-axis completes right-handed system.

\section{Propulsion Reference Frame $(\mathscr{F} P)$}

The propulsion reference frame is identified with index $P$. Its origin coincides with the CoM of the vehicle. It is generally related to the $\mathscr{F}_{B}$, since here the three components of the thrust force are defined, but sometimes it is useful to express the thrust force in spherical components with a magnitude and two direction angles. The

\footnotetext{
${ }^{1}$ Erwin Mooij, private communication (Sept 2017).
} 


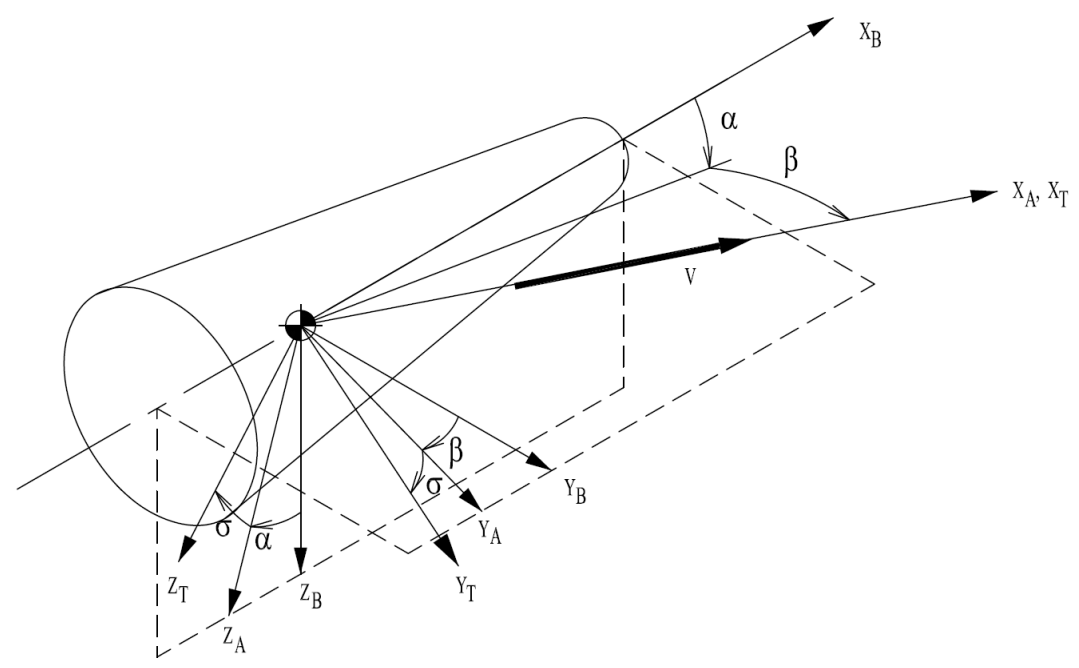

Figure 4.4: Graphical representation of the relationship between $B$-, $A$ - and $T$-frames. [Mooij, 2015]

$\mathscr{F}_{P}$, thus, is defined with a frame transformation of the $\mathscr{F}_{B}$ over two rotation angles. We obtain an $\mathrm{X}_{P}$-axis collinear with the thrust vector, and the $\mathrm{Y}_{P}$-axis and $\mathrm{Z}_{P}$-axis that follow from the transformation of the $\mathscr{F}_{B}$.

\subsection{State Variables}

The analysis of the variation of the geometrical state of a body with respect to a reference frame is studied by the kinematics. The motion of a body can be ideally divided into two main motions:

- point or translational motion: 3-DoF translational motion of a point mass

- rigid-body or rotational motion: 3-DoF rotational motion of a finite-sized body.

The information about the motion is formulated by means of mathematical variables, called state variables. The term state is used to indicate the kinematic characteristics of a body's motion by providing a complete description of position, velocity, attitude and angular rate. Several state variables exist and sometimes it is more appropriate to describe the motion in one specific set of state variables or in another one depending on the application involved. As a consequence, the motion is differently described depending on the chosen state variables. To achieve the objectives of this thesis project, the stability of the system and the performance of the controller will be analysed in single points along the trajectory. Thus, considering that for each of these points the position and the velocity is fixed, we are interested in describing the attitude and the angular rates about these nominal conditions. In the following, the state variables that are useful for our analysis are presented, on the basis of the standard definitions given by Mooij [1994].

\subsubsection{Position and Velocity}

The state variables generally used to describe the position and velocity of a vehicle are Cartesian components and spherical components. Position and velocity in Cartesian components are defined as following:

Position: $x, y, z$;

Velocity: $\dot{x}, \dot{y}, \dot{z}$.

These variables can be expressed in $\mathscr{F}_{I}$ and $\mathscr{F}_{R}$, indicated with the subscript " $I$ " or " $R$ ", respectively. Generally the velocity components in $\mathscr{F}_{R}$ are indicated with $u, v, w$, instead of $\dot{x}_{R}, \dot{y}_{R}, \dot{z}_{R}$.

Position and velocity in spherical components are defined as following:

Position: distance $R$, longitude $\tau$, latitude $\delta$;

Velocity: groundspeed $V$, flight-path angle $\gamma$, heading angle $\chi$.

These variables can be expressed in $\mathscr{F}_{R}$ as shown in Figure 4.5. The distance $R$ is the distance between the Earth's CoM and the vehicle's CoM. The longitude $\tau$ is defined as $0^{\circ} \leq \tau<360^{\circ}$, positive when measured to the east. The latitude $\delta$ is defined as $-90^{\circ} \leq \delta \leq 90^{\circ}$ and it is measured along a specified meridian starting at the 




Figure 4.5: Representation of the position $(R, \tau, \delta)$ and velocity $\left(V_{g}, \gamma_{g}, \chi_{g}\right)$ spherical components w.r.t. $\mathscr{F}_{R}$ [Mooij, 1998a].

equator, positive to the north and negative to the south. The groundspeed $V_{g}$ is expressed with respect to $\mathscr{F}_{R}$. The flight-path angle $\gamma_{g}$ is the angle between the velocity vector and the local horizontal plane $\left(-90^{\circ} \leq \gamma_{g} \leq\right.$ $90^{\circ}$ ), which is positive when the velocity vector is above the local horizon. The heading angle $\chi_{g}$ is the angle between the direction of the projection of the velocity vector in the local horizontal plane and the direction of the local north $\left(-180^{\circ} \leq \chi_{g}<180^{\circ}\right)$. For example, a heading angle of $90^{\circ}$ means that the vehicle is moving parallel to the equator in east direction.

The Cartesian coordinates are generally used for flight simulation as they are efficient and stable to be integrated numerically both in $\mathscr{F}_{I}$ or $\mathscr{F}_{R}$ and they do not present singularity. Another important advantage of using Cartesian coordinates is that the EoM in $\mathscr{F}_{I}$ are written in an elegant and compact set of differential EoM, differently from the EoM written in spherical coordinates. Indeed, they have several sine and cosine functions, which make the derivations very though and laborious. However, for control studies it is preferable to represent the vehicle's motion in spherical coordinates with respect to $\mathscr{F}_{R}$. The reason is that, when expressing the motion in the $\mathscr{F}_{I}$, the position vector of a landing site on the surface of the Earth will be continuously changing, because the Earth is rotating with respect to the $\mathscr{F}_{I}$. Moreover, describing the position in $x, y, z$ coordinates will not give a very intuitive interpretation of the kinetic state of the vehicle, which would be useful for stability analysis. Contrarily, the spherical components in $\mathscr{F}_{R}$ can clearly provide the location of a site with respect to the Earth. For instance, a level flight toward east is easily represented by the relative velocity of the vehicle with respect to the Earth, with $\gamma=0^{\circ}$ and $\chi=90^{\circ}$. The spherical components present singularities in the EoM at $\gamma= \pm 90^{\circ}$. However, this singularity is not encountered in the portion of the FSS-1 trajectory we are interested in (see Table 2.5).

From the above reasoning, it follows that it is convenient to use the Cartesian coordinates with respect to $\mathscr{F}_{I}$ for the simulation of vehicle's motion, and the spherical components with respect to the $\mathscr{F}_{R}$ for the stability and control studies. Moreover, it is always possible to convert the results from $\mathscr{F}_{I}$ to $\mathscr{F}_{R}$, and viceversa. Indeed, frame transformations are possible, as we will see in Section 4.4. Also, coordinate transformations are possible by means of a simple geometric relation between Cartesian and spherical coordinates (see Figure 4.5):

$$
\left(\begin{array}{l}
R \\
\delta \\
\tau
\end{array}\right)=\left(\begin{array}{c}
\sqrt{x^{2}+y^{2}+x^{2}} \\
\arcsin \frac{z}{R} \\
\arctan \frac{y}{x}
\end{array}\right) \longleftrightarrow\left(\begin{array}{l}
x \\
y \\
z
\end{array}\right)=\left(\begin{array}{c}
R \cos \delta \cos \tau \\
R \cos \delta \sin \tau \\
R \sin \delta
\end{array}\right)
$$

\subsubsection{Attitude and Angular Rate}

The position and velocity state variables completely describe the motion of a vehicle when it is considered to be a point of mass. For more complex application, such as attitude control, the simplification of point of mass is inadequate and the body has to be considered as a rigid body with 6-DoF. Consequently, state variables describing the vehicle's attitude and angular motion are needed and they are presented in the following paragraphs. 


\section{Attitude}

Generally, the attitude of a body is defined as the orientation of $\mathscr{F}_{B}$ with respect to an other reference frame. The most commonly used state variables to describe the vehicle's attitude are the classical Euler angles, aerodynamic angles (a special set of Euler angles), direction cosines, and quaternions.

According to the Euler's Rotation Theorem, any arbitrary rotation may be described by only three parameters in a three-dimensional space [Palais and Palais, 2007]. The classical Euler angles can represent the attitude of the body with respect to $\mathscr{F}_{I}$. They are the roll angle, $\phi$, the pitch angle, $\theta$, and the yaw angle, $\psi$. It is important to specify the order that is followed to execute the transformations and about which axes. There exist 12 possible sequences of rotation axis, which are subdivided into proper Euler angles and Tait-Bryan angles. The only difference is that Tait-Bryan angles represent rotations about three distinct axes (e.g. $x-y-z$ ), while proper Euler angles use the same axis for both the first and third elemental rotations (e.g., $z-x-z)$. The aerodynamic angles are a particular set of Tait-Bryan angles, where the sequence of transformations from $\mathscr{F}_{T}$ to $\mathscr{F}_{B}$ is with order $y-z-x$. This means that the sequence begins with a rotation about the $Y$-axis, followed by a rotation about the $Z$-axis, and concluded by a rotation about the $X$-axis. The direction cosines are the cosines of the angles enclosed between the axes of the two reference frames considered, resulting in a direction cosine matrix of dimension $3 \times 3$, with a total of 9 direction cosines. A quaternion is a 4 -dimensional hyper-complex number, consisting of one scalar and three vectors, that can completely define the attitude of a body.

For a robust simulation, it is essential to avoid singularities in the equations of motion. The sets of variables that are singularity-free, are those that describe a 3-dimensional motion in more than three parameters and consequently contain redundancy. Direction cosines present a six-fold redundancy and quaternions are single-redundant. The high degree of redundancy of the direction cosines makes their use laborious and inefficient from a computational point of view. For single redundancy of the quaternions, an additional constraint equation needs to be implemented. The Euler angles (including the aerodynamic angles) are convenient for attitude representation, as they are very intuitive and easy to visualise. However, they have the drawback of presenting at least one singularity at $\pm 90^{\circ}$ of one of the angles, depending on the sequence of rotation followed. By changing the rotation sequence the singularity can be shifted, but not avoided.

For flight and attitude simulation, it is convenient to use quaternions. They define an elegant set of linear equations of motion, convenient for integration and easy to handle. Moreover, their application in past simulation studies is well-documented in the literature, ensuring that they perform well for flight and attitude simulation. For attitude control and stability analysis, the state variables that are commonly used are the aerodynamic angles. The reason is that controllability and stability are mainly related to aerodynamic forces and moments. Also, the singularity is encountered when the sideslip angle is $\beta= \pm 90^{\circ}$, a condition that is never reached along the FSS-1 reference trajectory. A detailed description of aerodynamic angles and quaternions is given in the following.

\section{Aerodynamic Angles}

The aerodynamic angles are a convenient set of state variables for attitude control and stability analysis. They describe the attitude of a vehicle with respect to $\mathscr{F}_{T}$ or, in other words, the orientation of $\mathscr{F}_{B}$ with respect to $\mathscr{F}_{T}$. The aerodynamic angles are the angle of attack, $\alpha$, sideslip angle, $\beta$, and bank angle, $\sigma$. They can describe the vehicle's attitude with respect to groundspeed (subscript "g") or airspeed (subscript "a"). The aerodynamic angles are defined as: $-180^{\circ} \leq \alpha<+180^{\circ}$, with $\alpha>0^{\circ}$ for a nose-up attitude; $-90^{\circ} \leq \beta \leq+90^{\circ}$, with $\beta>0^{\circ}$ for a nose-left attitude; $-180^{\circ} \leq \sigma<+180^{\circ}$, with $\sigma>0^{\circ}$ when the vehicle is banked to the right. Figure 4.6 illustrates the aerodynamic angles in $\mathscr{F}_{B}$.

\section{Quaternions}

Quaternions constitute the most-used set of parameters that describe the attitude dynamics. A quaternion is a 4-dimensional hyper-complex number, consisting of four elements: the first three elements define the vector component of the quaternion and the fourth is the scalar component. It is defined as follows:

$$
\mathbf{q}=\left(\begin{array}{l}
\mathbf{q}_{\mathbf{v}} \\
q_{4}
\end{array}\right)=\left(\begin{array}{l}
q_{1} \\
q_{2} \\
q_{3} \\
q_{4}
\end{array}\right)=\left(\begin{array}{c}
n_{x} \sin \frac{\theta}{2} \\
n_{y} \sin \frac{\theta}{2} \\
n_{z} \sin \frac{\theta}{2} \\
\cos \frac{\theta}{2}
\end{array}\right)
$$

where $\mathbf{q}_{\mathbf{v}}$ is the vector component of the quaternion, $n_{x}, n_{y}$ and $n_{z}$ are the components of the rotation axis $\mathbf{n}$, and $\theta$ is the rotation angle about this axis.

Since quaternions provide information about the orientation of a rigid body by means of four quantities, they are single-redundant in the attitude description in $\mathbb{R}^{3}$. Thus, the four quaternions are not mutually inde- 


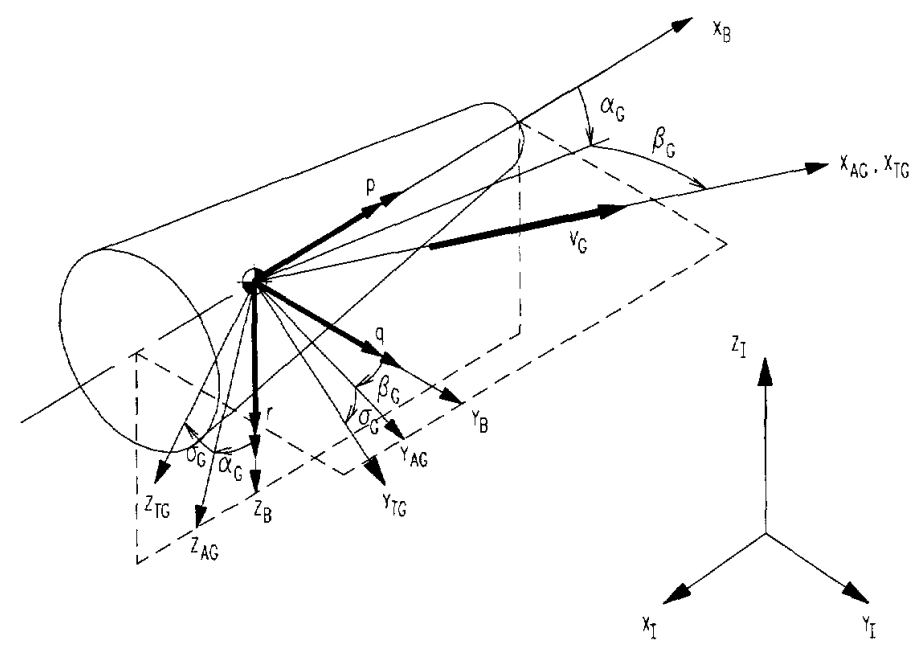

Figure 4.6: Representation of the aerodynamic angles $\left(\alpha_{g}, \beta_{g}\right.$, and $\left.\sigma_{g}\right)$ and angular rate components ( $p, q$, and $\left.r\right)$ [Mooij, 1998a].

pendent, but they are related by a constraint equation that, for attitude quaternions, is:

$$
\|\mathbf{q}\|=q_{1}^{2}+q_{2}^{2}+q_{3}^{2}+q_{4}^{2}=1
$$

which means that quaternions describe a sphere in the 4-dimensional space. Thus, any rotation is defined as a trajectory on the surface of the 3-dimensional sphere.

The algebraic operations with quaternions constitute a special algebra and, in addition, quaternions are characterized by peculiar properties. This represents a very vast topic, that the interested reader can learn about from several textbooks covering this topic, for example, Kuipers [2002].

\section{Angular Rate}

The vehicle's angular rate, whose components are expressed along the body axes, is the rotational velocity of $\mathscr{F}_{B}$ with respect to $\mathscr{F}_{I}$. The rotation vector $\omega$ is defined as:

$$
\omega=(p, q, r)^{T}
$$

with $p$ being the roll rate, $q$ being the pitch rate, and $r$ being the yaw rate along the $X_{B^{-}}, Y_{B^{-}}, Z_{B^{-}}$-axis, respectively. A representation of the angular rate components with respect to $\mathscr{F}_{I}$ is given in Figure 4.6.

The quaternions time derivative is formulated with the following differential equation [Kuipers, 2002]:

$$
\dot{\mathbf{q}}=\frac{1}{2} \mathbf{q} \cdot \omega
$$

that can be written in matrix form as:

$$
\dot{\mathbf{q}}=\frac{1}{2} \Xi(\mathbf{q}) \omega
$$

where

$$
\Xi(\mathbf{q})=\left[\begin{array}{c}
q_{4} \mathbf{I}_{3 x 3}+\left[\mathbf{q}_{\mathbf{v}} \times\right] \\
-\mathbf{q}^{T}
\end{array}\right], \quad\left[\mathbf{q}_{\mathbf{v}} \times\right]=\left[\begin{array}{ccc}
0 & -q_{3} & q_{2} \\
q_{3} & 0 & -q_{1} \\
-q_{2} & q_{1} & 0
\end{array}\right]
$$

with $\mathbf{I}_{3 \times 3}$ being a $3 \times 3$ identify matrix and $\left[\mathbf{q}_{\mathbf{v}} \times\right]$ indicating the cross product matrix.

\subsection{Frame Transformations}

Transformations between reference frames are essential to describe the motion of a vehicle rigorously: in case some forces and moments are defined in different reference frames, they need to be expressed with respect to the same frame in the equations of motion. Mooij [1994] offers the definition of the frame transformation. 
Table 4.1: Standard frame transformations. [Mooij, 1994]

\begin{tabular}{|c|c|c|}
\hline \multicolumn{3}{|c|}{ Standard Frame Transformation } \\
\hline From $\rightarrow$ To & Transformation Matrix & Specifications \\
\hline $\mathscr{F}_{R} \rightarrow \mathscr{F}_{I}$ & $\mathbf{C}_{\mathbf{I}, \mathbf{R}}=\mathbf{C}_{\mathbf{z}}\left(-\omega_{c b} t\right)$ & $\begin{array}{c}\omega_{c b}: \text { Earth rotational rate }[\mathrm{rad} / \mathrm{s}] \\
t: \text { time from epoch }[\mathrm{s}]\end{array}$ \\
\hline $\mathscr{F}_{V} \rightarrow \mathscr{F}_{R}$ & $\mathbf{C}_{\mathbf{R}, \mathbf{V}}=\mathbf{C}_{\mathbf{z}}(-\tau) \mathbf{C}_{\mathbf{y}}\left(\frac{\pi}{2}+\delta\right)$ & $\begin{array}{c}\tau: \text { Earth-centred longitude [rad] } \\
\delta: \text { Earth-centred latitude [rad] }\end{array}$ \\
\hline $\mathscr{F}_{W} \rightarrow \mathscr{F}_{V}$ & $\mathbf{C}_{\mathbf{V}, \mathbf{W}}=\mathbf{C}_{\mathbf{z}}\left(-\chi_{w}\right) \mathbf{C}_{\mathbf{y}}\left(-\gamma_{w}\right)$ & $\begin{array}{c}\chi_{w}: \text { flight-path angle for wind vector [rad] } \\
\gamma_{w}: \text { heading angle for wind vector [rad] }\end{array}$ \\
\hline $\mathscr{F}_{T G} \rightarrow \mathscr{F}_{V}$ & $\mathbf{C}_{\mathbf{V}, \mathbf{T G}}=\mathbf{C}_{\mathbf{z}}\left(-\chi_{g}\right) \mathbf{C}_{\mathbf{y}}\left(-\gamma_{g}\right)$ & $\begin{array}{c}\chi_{g}: \text { flight-path angle for groundspeed [rad] } \\
\gamma_{g}: \text { heading angle for groundspeed [rad] }\end{array}$ \\
\hline $\mathscr{F}_{T A} \rightarrow \mathscr{F}_{V}$ & $\mathbf{C}_{\mathbf{V}, \mathbf{T A}}=\mathbf{C}_{\mathbf{z}}\left(-\chi_{a}\right) \mathbf{C}_{\mathbf{y}}\left(-\gamma_{a}\right)$ & $\begin{array}{c}\chi_{a}: \text { flight-path angle for airspeed [rad] } \\
\gamma_{a}: \text { heading angle for airspeed [rad] }\end{array}$ \\
\hline $\mathscr{F}_{A A} \rightarrow \mathscr{F}_{T A}$ & $\mathbf{C}_{\mathbf{T A}, \mathbf{A A}}=\mathbf{C}_{\mathbf{X}}\left(\sigma_{a}\right)$ & $\sigma_{a}:$ bank angle based on airspeed [rad] \\
\hline $\mathscr{F}_{B} \rightarrow \mathscr{F}_{A A}$ & $\mathbf{C}_{\mathbf{A A}, \mathbf{B}}=\mathbf{C}_{\mathbf{z}}\left(\beta_{a}\right) \mathbf{C}_{\mathbf{y}}\left(-\alpha_{a}\right)$ & $\begin{array}{c}\beta_{a}: \text { sideslip angle for airspeed [rad] } \\
\alpha_{a}: \text { angle of attack for airspeed [rad] }\end{array}$ \\
\hline $\mathscr{F}_{B} \rightarrow \mathscr{F}_{A G}$ & $\mathbf{C}_{\mathbf{A G}, \mathbf{B}}=\mathbf{C}_{\mathbf{z}}\left(\beta_{g}\right) \mathbf{C}_{\mathbf{y}}\left(-\alpha_{g}\right)$ & $\begin{array}{c}\beta_{g}: \text { sideslip angle for groundspeed [rad] } \\
\alpha_{g}: \text { angle of attack for groundspeed [rad] }\end{array}$ \\
\hline $\mathscr{F}_{B} \rightarrow \mathscr{F}_{P}$ & $\mathbf{C}_{\mathbf{P}, \mathbf{B}}=\mathbf{C}_{\mathbf{y}}\left(\epsilon_{T}\right) \mathbf{C}_{\mathbf{z}}\left(\psi_{T}\right)$ & $\begin{array}{l}\epsilon_{T}: \text { thrust elevation angle [rad] } \\
\psi_{T}: \text { thrust azimuth angle [rad] }\end{array}$ \\
\hline
\end{tabular}

Considering a vector $\mathbf{v}_{\mathbf{A}}$ defined in a generic frame $A, \mathscr{F}_{A}$. The same vector can be described in a frame $B, \mathscr{F}_{B}$, by means of a rotation and a translation, as follows:

$$
\mathbf{v}_{\mathbf{B}}=\mathbf{T}+\mathbf{C}_{\mathbf{B}, \mathbf{A}} \mathbf{v}_{\mathbf{A}}
$$

with $\mathbf{T}$ being the translation vector from the origin of $\mathscr{F}_{A}$ to the origin of $\mathscr{F}_{B}$ and $\mathbf{C}_{\mathbf{B}, \mathbf{A}}$ being the transformation matrix defining the rotation from $\mathscr{F}_{A}$ to $\mathscr{F}_{B}$.

Any rotation from $\mathscr{F}_{A}$ to $\mathscr{F}_{B}$, both right-handed Cartesian frames, can be written as a combination of matrices representing unit-axis rotations, which are $\mathbf{C}_{\mathbf{x}}, \mathbf{C}_{\mathbf{y}}$ and $\mathbf{C}_{\mathbf{z}}$ about the $X, Y$ and $Z$ axes, respectively. A definition of unit axis rotations can be provided for an arbitrary angle $\alpha$ :

$$
\mathbf{C}_{\mathbf{x}}(\alpha)=\left[\begin{array}{ccc}
1 & 0 & 0 \\
0 & \cos \alpha & \sin \alpha \\
0 & -\sin \alpha & \cos \alpha
\end{array}\right], \quad \mathbf{C}_{\mathbf{y}}(\alpha)=\left[\begin{array}{ccc}
\cos \alpha & 0 & -\sin \alpha \\
0 & 1 & 0 \\
\sin \alpha & 0 & \cos \alpha
\end{array}\right], \quad \mathbf{C}_{\mathbf{z}}(\alpha)=\left[\begin{array}{ccc}
\cos \alpha & \sin \alpha & 0 \\
-\sin \alpha & \cos \alpha & 0 \\
0 & 0 & 1
\end{array}\right]
$$

These matrices are orthonormal, thus also their product is orthonormal. A property of an orthonormal matrix is that its inverse equals its transpose, thus the transformation matrix from $\mathscr{F}_{B}$ to $\mathscr{F}_{A}$ can be determined if the transformation matrix from $\mathscr{F}_{A}$ to $\mathscr{F}_{B}$ is known, i.e.:

$$
\mathbf{C}_{\mathbf{A}, \mathbf{B}}=\mathbf{C}_{\mathbf{B}, \mathbf{A}}^{-1}=\mathbf{C}_{\mathbf{B}, \mathbf{A}}^{\mathbf{T}}
$$

As we mentioned above, any rotation can be written as a combination of matrices $\mathbf{C}_{\mathbf{x}}, \mathbf{C}_{\mathbf{y}}$ and $\mathbf{C}_{\mathbf{z}}$. In Table 4.1, the transformation between the standard reference frames are reported. The full derivation can be found in Mooij [1994]. From the main transformation matrices it is possible to deduce all the other transformation matrices by means of a combination of them. For instance, for a transformation from the body reference frame $\mathscr{F}_{B}$ to $\mathscr{F}_{R}, \mathbf{C}_{\mathbf{R}, \mathbf{B}}$ can be defined as:

$$
\mathrm{C}_{\mathrm{R}, \mathrm{B}}=\mathrm{C}_{\mathrm{R}, \mathrm{V}} \mathrm{C}_{\mathrm{V}, \mathrm{AG}} \mathrm{C}_{\mathrm{AG}, \mathrm{B}}
$$

Once the transformation matrices are specified, it is possible to derive the coordinate transformation from quaternions to aerodynamic angles, and viceversa. It can be done starting from the consideration that quater- 
nions identify the orientation of $\mathscr{F}_{B}$ with respect to $\mathscr{F}_{I}$. This orientation can be expressed also with an orthogonal matrix, defined as:

$$
\mathbf{C}_{\mathbf{I}, \mathbf{B}}=\left[\begin{array}{ccc}
1-2\left(q_{2}^{2}+q_{3}^{2}\right) & 2\left(q_{1} q_{2}-q_{3} q_{4}\right) & 2\left(q_{1} q_{3}+q_{2} q_{4}\right) \\
2\left(q_{1} q_{2}+q_{3} q_{4}\right) & 1-2\left(q_{1}^{2}+q_{3}^{2}\right) & 2\left(q_{2} q_{3}-q_{1} q_{4}\right) \\
2\left(q_{1} q_{3}-q_{2} q_{4}\right) & 2\left(q_{2} q_{3}+q_{1} q_{4}\right) & 1-2\left(q_{1}^{2}+q_{2}^{2}\right)
\end{array}\right]
$$

Also, the aerodynamic angles describe the orientation of $\mathscr{F}_{B}$ with respect to $\mathscr{F}_{T}$, which can be identified by the following transformation matrix:

$$
\mathbf{C}_{\mathbf{T}, \mathbf{B}}=\mathbf{C}_{\mathbf{x}}(\sigma) \mathbf{C}_{\mathbf{z}}(\beta) \mathbf{C}_{\mathbf{y}}(-\alpha) \quad \text { with } \quad \mathbf{C}_{\mathbf{T}, \mathbf{B}}=\mathbf{C}_{\mathbf{T}, \mathbf{V}} \mathbf{C}_{\mathbf{V}, \mathbf{I}} \mathbf{C}_{\mathbf{I}, \mathbf{B}}
$$

where $\mathbf{C}_{\mathbf{T}, \mathbf{B}}$ is known because $\mathbf{C}_{\mathbf{T}, \mathbf{V}}$ and $\mathbf{C}_{\mathbf{V}, \mathbf{I}}$ are known for known flight parameters and $\mathbf{C}_{\mathbf{I}, \mathbf{B}}$ can be computed as shown before. So, this matrix multiplication yields:

$$
\mathbf{C}_{\mathbf{T}, \mathbf{B}}=\left[\begin{array}{ccc}
\cos \alpha \cos \beta & \sin \beta & \sin \alpha \cos \beta \\
-\cos \alpha \sin \beta \cos \sigma \sin \alpha \sin \sigma & \cos \beta \cos \sigma & -\sin \alpha \sin \beta \cos \sigma+\cos \alpha \sin \sigma \\
\cos \alpha \sin \beta \sin \sigma-\sin \alpha \cos \sigma & -\cos \beta \sin \sigma & \sin \alpha \sin \beta \sin \sigma+\cos \alpha \cos \sigma
\end{array}\right]
$$

from which the aerodynamic angles can be computed:

$$
\alpha=\operatorname{atan}\left(\frac{\mathbf{C}_{\mathbf{T}, \mathbf{B}}(1,3)}{\mathbf{C}_{\mathbf{T}, \mathbf{B}}(1,1)}\right), \quad \beta=\operatorname{asin}\left(\mathbf{C}_{\mathbf{T}, \mathbf{B}}(1,2)\right), \quad \sigma=\operatorname{atan}\left(-\frac{\mathbf{C}_{\mathbf{T}, \mathbf{B}}(3,2)}{\mathbf{C}_{\mathbf{T}, \mathbf{B}}(2,2)}\right)
$$

with $\alpha=\left[-\frac{\pi}{2}, \frac{\pi}{2}\right], \beta=[-\pi, \pi], \sigma=[-\pi, \pi]$.

From the combination of the defined matrices, also the coordinate transformation from aerodynamic angles to quaternions can be defined as [NASA, 1977]:

$$
\left(\begin{array}{l}
q_{1} \\
q_{2} \\
q_{3} \\
q_{4}
\end{array}\right)=\left(\begin{array}{c}
-\sin \frac{\alpha}{2} \sin \frac{\beta}{2} \cos \frac{\sigma}{2}+\cos \frac{\alpha}{2} \cos \frac{\beta}{2} \sin \frac{\sigma}{2} \\
-\sin \frac{\alpha}{2} \cos \frac{\beta}{2} \cos \frac{\sigma}{2}+\cos \frac{\alpha}{2} \sin \frac{\beta}{2} \sin \frac{\sigma}{2} \\
\sin \frac{\alpha}{2} \cos \frac{\beta}{2} \sin \frac{\sigma}{2}+\cos \frac{\alpha}{2} \sin \frac{\beta}{2} \cos \frac{\sigma}{2} \\
\sin \frac{\alpha}{2} \sin \frac{\beta}{2} \sin \frac{\sigma}{2}+\cos \frac{\alpha}{2} \cos \frac{\beta}{2} \cos \frac{\sigma}{2}
\end{array}\right)
$$

\subsection{Force Models}

External forces and moments act on the vehicle as a result of the environment where the vehicle is operating. The main forces and moments acting on a spaceplane in hypersonic ascent trajectory are of gravitational, aerodynamic and propulsion origin. Even if the propulsion forces are considered as external forces, they contain also the internal forces due to mass variation, i.e., Coriolis and relative forces and moments. There are other external forces and moments, such as those due to the magnetic field and third body perturbations, but for our application they are negligible with respect to the main ones.

Gravitational, aerodynamic, and propulsion forces and moments influence the motion of a spaceplane along its trajectory and, consequently, its flying qualities and controllability characteristics. It should be noticed that the external forces influence the translational motion, while the external moments affect the rotational motion. However, when the external forces are not acting on the CoM of the vehicle, they generate a moment about the CoM. The gravitational model has been already treated in Section 3.1. The aerodynamic and propulsion models are presented in the following sections.

\subsubsection{Aerodynamics}

The aerodynamic force vector is defined with respect to $\mathscr{F}_{A A}$ :

$$
\mathbf{F}_{\mathbf{a}, \mathbf{A A}}=\left(\begin{array}{c}
-D \\
-S \\
-L
\end{array}\right)=\bar{q} S_{r e f}\left(\begin{array}{l}
-C_{D} \\
-C_{S} \\
-C_{L}
\end{array}\right)
$$

where the components, drag force $D$, side force $S$ and lift force $L$, are positive along the $-X_{A A},-Y_{A A}$, and $-Z_{A A}$ axes, respectively, as Figure 4.7 shows. The term $\bar{q}=\frac{1}{2} \rho V_{a}^{2}$ is the dynamic pressure $\left[\mathrm{N} / \mathrm{m}^{2}\right], \rho$ the air density $\left[\mathrm{kg} / \mathrm{m}^{3}\right], V_{a}$ the vehicle velocity with respect to the atmosphere $[\mathrm{m} / \mathrm{s}], S_{\text {ref }}$ the reference surface $\left[\mathrm{m}^{2}\right]$, and $C_{D}$, 


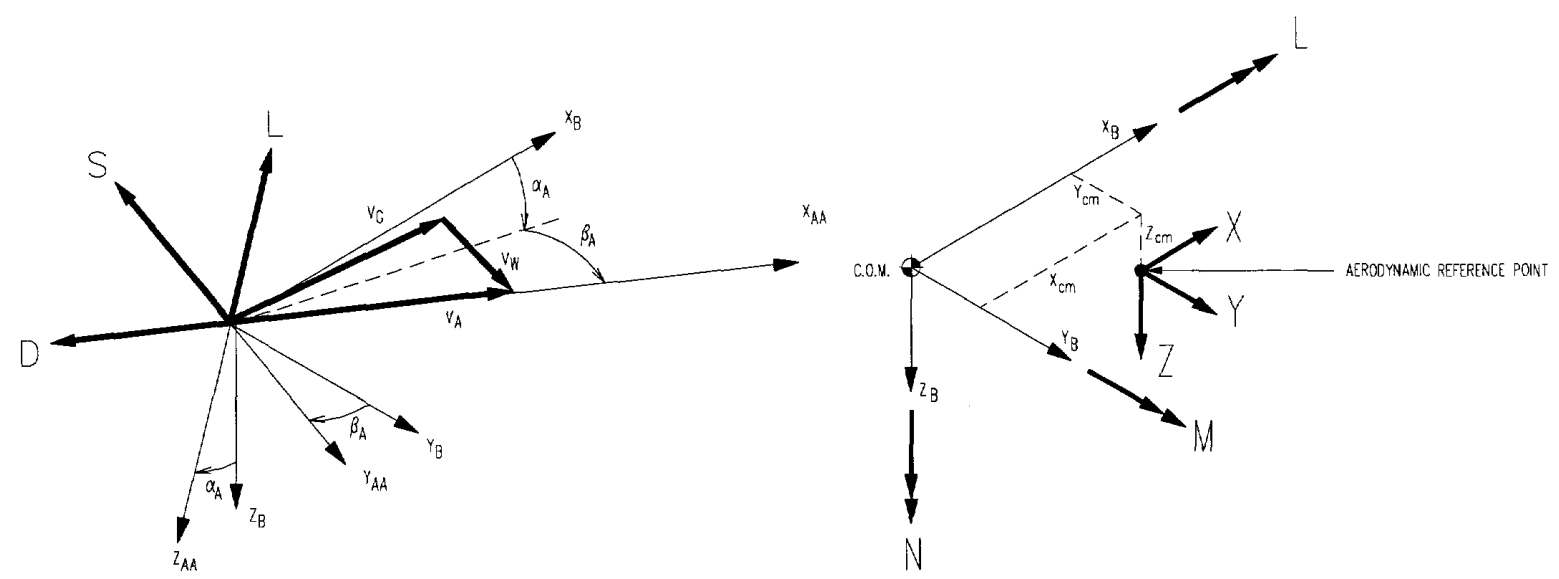

Figure 4.7: Left: aerodynamic forces in $\mathscr{F}_{A A}$. Right: aerodynamic moments in $\mathscr{F}_{B}$ and moments due to aerodynamic forces not applied to the CoM: $(L, M, N)^{T}=\left(x_{c m}, y_{c m}, z_{c m}\right)^{T} \times(X, Y, Z)^{T}$ [Mooij, 1998a].

$C_{S}$, and $C_{L}$ the drag, side-force, and lift coefficient, respectively. These coefficients are function of the Mach number and the aerodynamic angles $\alpha_{a}$ and $\beta_{a}$ and they can be evaluated by means of simulations, windtunnel and in-flight measurements.

The aerodynamic moment vector is defined with respect to $\mathscr{F}_{B}$ :

$$
\mathbf{M}_{\mathbf{a}, \mathbf{B}}^{\mathbf{M}}=\left(\begin{array}{c}
\mathscr{L} \\
\mathscr{M} \\
\mathscr{N}
\end{array}\right)=\bar{q} S_{\text {ref }}\left(\begin{array}{c}
b_{r e f} C_{l} \\
c_{r e f} C_{m} \\
b_{r e f} C_{n}
\end{array}\right)
$$

with the rolling moment $\mathscr{L}$, the pitching moment $\mathscr{M}$, and the yawing moment $\mathscr{N}$ being the components. The reference length is the wing span $b_{r e f}[\mathrm{~m}]$ for the roll and yaw moments and the mean aerodynamic wing chord $c_{r e f}[\mathrm{~m}]$ for the pitch moment. $C_{l}, C_{m}$, and $C_{n}$ are the roll, pitch, and yaw moment coefficient, respectively. The definition of the aerodynamic coefficients and further details about the aerodynamic properties of our reference vehicle are presented in Section 2.1.1.

Figure 4.7 shows the aerodynamic forces in $\mathscr{F}_{A A}$, the components of the aerodynamic moments in $\mathscr{F}_{B}$ and the aerodynamic forces that are computed with respect to the aerodynamic reference point. When the location of the aerodynamic reference point does not coincide with the CoM of the vehicle, the forces give a contribution to the aerodynamic moment. Considering the location of the CoM equal to $\mathbf{r}_{\mathbf{c m}}=\left(x_{c m}, y_{c m}, z_{c m}\right)^{T}$ with respect to $\mathscr{F}_{B}$, the total aerodynamic moment is:

$$
\mathbf{M}_{\mathbf{a}, \mathbf{B}}=\mathbf{M}_{\mathbf{a}, \mathbf{B}}^{\mathrm{M}}+\mathbf{M}_{\mathbf{a}, \mathbf{B}}^{\mathbf{F}}=\mathbf{M}_{\mathbf{a}, \mathbf{B}}^{\mathrm{M}}+\mathbf{r}_{\mathbf{c m}} \times \mathbf{F}_{\mathbf{a}, \mathbf{B}}
$$

where $\mathbf{M}_{\mathbf{a}, \mathbf{B}}^{\mathbf{M}}$ is the moment vector of the aerodynamic moments and $\mathbf{M}_{\mathbf{a}, \mathbf{B}}^{\mathbf{F}}=\mathbf{r}_{\mathbf{c m}} \times \mathbf{F}_{\mathbf{a}, \mathbf{B}}$ is the moment vector due to the aerodynamic forces. Since the aerodynamic force vector has been defined in $\mathscr{F}_{A A}, \mathbf{F}_{\mathbf{a}, \mathbf{A A}}$ needs to be transformed into $\mathbf{F}_{\mathbf{a}, \mathbf{B}}$ in $\mathscr{F}_{B}$ thanks to the transformation matrix $\mathbf{C}_{\mathbf{B}, \mathbf{A A}}=\mathbf{C}_{\mathbf{y}}\left(\alpha_{a}\right) \mathbf{C}_{\mathbf{z}}\left(-\beta_{a}\right)$ (see Section 4.4) as follows:

$$
\mathbf{F}_{\mathbf{a}, \mathbf{B}}=\mathbf{C}_{\mathbf{B}, \mathbf{A A}} \mathbf{F}_{\mathbf{a}, \mathbf{A A}}
$$

\subsubsection{Propulsion}

System requirement $S$-3 defines that rocket engines shall be used to propel the FSS-1 reference vehicle along its ascent trajectory. This means that mass varies during the flight affecting the motion of the vehicle. For the Principle of Solidification, the Coriolis and the relative forces and moments have to be added to the external forces and moments to account for the effect of mass variations. The Coriolis and relative forces and moments can be deduced from the information provided by Mooij [1998a].

Considering the rate of mass expulsion $\dot{m}$ that enters the exit area of the nozzle $A_{e}$ with a velocity $\mathbf{V}_{\mathbf{T}}$ (collinear to the thrust vector $\mathbf{T}$ ) at the centre of mass flow, which is located from the $\mathscr{F}_{B}$ origin at a distance $\mathbf{r}_{\mathbf{T}}$, the Coriolis and relative forces are defined as following:

$$
\begin{gathered}
\mathbf{F}_{\mathbf{C}}=2 \boldsymbol{\omega} \times\left(\dot{m} \mathbf{r}_{\mathbf{T}}\right) \\
\mathbf{F}_{\mathbf{r e l}}=\dot{m} \mathbf{V}_{\mathbf{T}}
\end{gathered}
$$


with $\dot{m}=-\frac{\mathrm{d} m(t)}{\mathrm{d} t}$ being the mass variation rate and $m(t)$ being the total mass of the vehicle at instant $t$. We assume that the mass variation is due to only propellant expulsion, thus $\dot{m}$ can be also seen as the propellant mass flow. Thus, the minus sign is used to make $\dot{m}$ positive, since the mass is decreasing $\left(\frac{\mathrm{d} m(t)}{\mathrm{d} t}<0\right)$.

The physical meaning of the Coriolis force is the following. Considering that the centre of mass flow is located along the $X_{B}$-axis, the Coriolis force is generated from rotations about $Y_{B}$-axis and $Z_{B}$-axis. These rotations are usually very small, thus $\mathbf{F}_{\mathbf{C}}$ is much smaller than $\mathbf{F}_{\text {rel }}$ and it can be usually neglected even if the rotational velocity about the $\mathrm{X}_{B}$-axis is high. Moreover, $\mathbf{F}_{\mathbf{C}}$ can be neglected even if the centre of mass flow is not along the $\mathrm{X}_{B}$-axis, because the roll rate during the manoeuvres are generally small [Mooij, 1998a].

The Coriolis and relative moments are defined as:

$$
\begin{gathered}
\mathbf{M}_{\mathbf{C}}=-\frac{\delta \mathbf{I}}{\delta t} \cdot \boldsymbol{\omega}+\dot{m} \mathbf{r}_{\mathbf{T}} \times\left(\boldsymbol{\omega} \times \mathbf{r}_{\mathbf{T}}\right) \\
\mathbf{M}_{\mathbf{r e l}}=\dot{m} \mathbf{r}_{\mathbf{T}} \times \mathbf{V}_{\mathbf{T}}
\end{gathered}
$$

The first term in the formula of the Coriolis moment is due to the change of inertia properties, while the second term is a damping moment generated by the exhaust jet and it is known as jet damping moment. The inertia variation is neglected in this application because the analyses that need to be conducted along the ascent trajectory, where the propulsive forces and moments act on the system, require the study of the rotational motion only at the design points. Thus, in these points the inertia properties can be considered constant, as well as the mass properties. The magnitude of $\mathbf{M}_{\mathbf{C}}$ is of the same order of the thrust misalignment and aerodynamic moments, thus it is usually not negligible. The relative moment is due to the impulse thrust and it will include any moment generated by thrust misalignment.

The thrust force can be defined as the summation of the impulse thrust and the pressure thrust:

$$
\mathbf{T}=\mathbf{T}_{\text {impulse }}+\mathbf{T}_{\text {pressure }}=\dot{m} \mathbf{V}_{\mathbf{T}}+\left(p_{e}-p_{a}\right) A_{e} \hat{\mathbf{r}}_{\mathbf{T}}
$$

with $p_{a}$ the ambient pressure and $p_{e}$ being the pressure $\left[\mathrm{N} / \mathrm{m}^{2}\right]$ in the flow at the exit area $A_{e}\left[\mathrm{~m}^{2}\right], \hat{\mathbf{r}}_{\mathrm{T}}$ is the unit vector directed from the vehicle's CoM to the centre of mass flow. The pressure difference between the pressure at the exit of the nozzle, $p_{e}$, and the ambient pressure, $p_{a}$ leads us to understand mathematically that there is an effect of interaction between the flow pushing against the atmosphere and generating the thrust and the motion of the vehicle. In hypersonic conditions, the pressure at the exit of the nozzle is generally much larger than the ambient pressure (typically they differ two orders of magnitude), thus it is common use to approximate $p_{e}-p_{a} \approx p_{e}$ in the formula of $\mathbf{T}$. From the definition of the relative force $\mathbf{F}_{\text {rel }}$ given above, it can be noticed that it is equal to the impulse thrust, meaning that it is an internal force. However, since the pressure thrust is an external force, we can conclude that thrust is a combination of internal and external forces.

The FSS-1 spaceplane has eight engines. Each engine generates a propulsion force that contributes to the motion of the vehicle's CoM and about the CoM. The propulsion force of the $i$-th engine is described by its magnitude, $T_{i}$, and the location of the engine with respect to the CoM, $\mathbf{r}_{\mathbf{T}, \mathbf{i}}$. The direction of the thrust vector with respect to $\mathscr{F}_{B}$ is identified by two direction angles: the thrust elevation $\epsilon_{T, i}$ and azimuth $\psi_{T, i}$. Thus, the total thrust force is given by the vectorial summation of the propulsion forces generated by all the engines. As a result, the total thrust is applied at the centre of thrust (CoT), which is distant $\mathbf{r}_{\mathbf{T}}$ from the CoM, and it is defined by the total magnitude $T$ and effective direction given by the angles $\epsilon_{T}$ and $\psi_{T}$.

When the CoT does not coincide with the CoM, the thrust force of the $i$-th engine in $\mathscr{F}_{B}, \mathbf{F}_{\mathbf{T}, \mathbf{B}}^{\mathbf{i}}$, induces a moment about the CoM, that is defined as:

$$
\mathbf{M}_{\mathrm{T}, \mathbf{B}}^{\mathbf{i}}=\mathbf{r}_{\mathrm{T}, \mathbf{i}} \times \mathbf{F}_{\mathrm{T}, \mathbf{B}}^{\mathbf{i}}
$$

The total thrust moment is calculated by taking the sum of $\mathbf{M}_{\mathbf{T}, \mathbf{B}}^{\mathbf{i}}$ of all the $n$ engines:

$$
\mathbf{M}_{\mathbf{T}, \mathbf{B}}=\sum_{i=1}^{n} \mathbf{M}_{\mathbf{T}, \mathbf{B}}^{\mathbf{i}}
$$

To calculate the thrust magnitude, the specific impulse $I_{s p}$ can be used. To get rid of the pressure term in the thurst expression the effective exhaust velocity, $V_{\text {eff }}$, is used: $T=\dot{m} V_{T}+\left(p_{e}-p_{a}\right) A_{e}=\dot{m} V_{e f f}$. Turner [2005] gives a definition of specific impulse, as the impulse given to the rocket by unit weight of propellant. So, it is a measure of the efficiency of the rocket engine. It can be formulated as follows:

$$
I_{s p}=\frac{T}{\dot{m} g_{0}}=\frac{V_{e f f}}{g_{0}}
$$


from which a simplified expression of thrust can be derived:

$$
\mathbf{T}=\dot{m} g_{0} I_{s p} \hat{\mathbf{T}}
$$

where $g_{0}=9.80665 \mathrm{~m} / \mathrm{s}^{2}$ is the standard gravity and $\hat{\mathbf{T}}$ indicates the direction of the thrust.

\subsection{Equations of Motion: General Formulation}

While kinematics studies the motion of a body irrespective of what causes it, dynamics studies the forces and moments and their effects on the motion of a body. Kinematics and dynamics can be described by differential equations, which together define the equations of motion of a body.

The EoM are formulated using the theory of the classical mechanics, specifically on the basis of the wellknown Newton's laws (Wakker [2015] p. 1):

First Law: $\quad$ "Every particle continues in its state of rest or uniform motion in a straight line relative to an inertial reference frame, unless it is compelled to change that state by forces acting upon it."

Second Law: "The time rate of change of linear momentum of a particle relative to an inertial reference frame is proportional to the resultant of all forces acting upon that particle and is collinear with and in the direction of the resultant force."

Third Law: $\quad$ "If two particles exert forces on each other, these forces are equal in magnitude and opposite in direction (action = reaction)."

The motion of a non-elastic body, as a spaceplane, can be divided into the motion of its CoM and the motion around its CoM. Thus, the motion of the vehicle has 6 DoF, 3 for translational motion and 3 for rotational motion. Consequently, the EoM comprehend a set of three equations, known as equations of the translational motion, describing position and velocity of the body, and a set of three equations, known as equations of rotational motion, which give information about the angular rate and attitude of the vehicle. Mission requirement $M-3$ states that the flying qualities and controllability of the reference vehicle shall be analysed in the design points with $M=5,10,15,20$ along the hypersonic ascent and re-entry trajectory. Thus, the analysis needs to be focused on the attitude and the rotational rate of the vehicle around the nominal conditions of these points, where position and velocity are fixed. In other words, we are willing to study the rotational motion of the FSS-1 spaceplane. However, for the verification of the aerodynamic database the translational motion is needed, to verify that FSS-1 is able to fly the HORUS re-entry trajectory. Also, both the translational and rotational EoM will be useful to derive the full set of linearised EoM that will be necessary for the stability analyses. Thus, both the translational and rotational EoM are relevant for this thesis project.

As discussed in Section 4.1, an EoM set as accurate as possible is required to perform the simulation. The non-linear set is appropriate for this scope with the assumptions of oblate Earth that rotates with a constant angular velocity $\omega_{\boldsymbol{R}}=\left(0,0, \omega_{c b}\right)^{T}$. To perform the stability and controllability analyses, a linearised set of EoM is convenient. In this case, the oblateness and the rotation of the Earth have a second-order effect, thus a spherical non-rotating $\left(\omega_{c b}=0 \mathrm{rad} / \mathrm{s}\right)$ Earth can be assumed.

For the system requirement S-3, we have that our reference vehicle is provided with a rocket propulsion system along its ascent trajectory. Thus, the mass of the vehicle changes during its motion. The equations of motion shall account for this effect. The Principle of Solidification states [Cornelisse et al., 1979] that:

"In general, equations of translational and rotational motion of an arbitrary variable mass system at time $\mathrm{t}$ can be written as the translational and rotational equations for a rigid body with mass $\mathrm{M}$ equal to the mass of the system at time $\mathrm{t}$, while in addition to the true external forces and moments, two apparent forces and moments are applied: the Coriolis and relative forces and moments, respectively."

The successive paragraphs define the EoM of a vehicle of variable mass $m$, located at distance $\mathbf{r}_{\mathbf{c m}}$ from the $\mathscr{F}_{I}$ origin, moving about the Earth with a angular rate $\boldsymbol{\omega}$ and a velocity $\mathbf{V}_{\mathbf{I}}$ with respect to $\mathscr{F}_{I}$. The vehicle is subjected to external forces of gravitational, propulsion and aerodynamic origin, which were presented before.

\subsubsection{Equations of the Translational Motion}

From the second Newton's law, the translational motion with respect to $\mathscr{F}_{I}$ is determined as follows:

$$
\tilde{\mathbf{F}}_{\mathbf{I}}=\mathbf{F}_{\mathbf{I}}+\mathbf{F}_{\mathbf{C}}+\mathbf{F}_{\text {rel }}=m \frac{d^{2} \mathbf{r}_{\mathbf{c m}}}{d t^{2}}
$$


where $\tilde{\mathbf{F}}_{\mathbf{I}}$ is the pseudo external force vector $[\mathrm{N}], \mathbf{F}_{\mathbf{I}}$ is the sum of the external forces acting on the vehicle $[\mathrm{N}]$, $\mathbf{F}_{\mathbf{C}}$ is the Coriolis force $[\mathrm{N}]$, and $\mathbf{F}_{\mathbf{r e l}}$ is the relative force $[\mathrm{N}]$, which were defined in Section 4.5. The term $\frac{d^{2} \mathbf{r}_{\mathbf{c m}}}{d t^{2}}$ is the acceleration $\left[\mathrm{m} / \mathrm{s}^{2}\right]$ of the vehicle's CoM with respect to $\mathscr{F}_{I}$, where $\mathbf{r}_{\mathbf{c m}}=\left(x_{c m}, y_{c m}, z_{c m}\right)$ represents the position of the vehicle's CoM with respect to the $\mathscr{F}_{I}$ origin in Cartesian components. $\mathbf{F}_{\mathbf{C}}$ and $\mathbf{F}_{\text {rel }}$ are due to the variation of mass distribution with time and they are equal to:

$$
\begin{gathered}
\mathbf{F}_{\mathbf{C}}=-2 \boldsymbol{\omega} \times \int_{m} \frac{\partial \tilde{\mathbf{r}}}{\partial t} d m \\
\mathbf{F}_{\mathbf{r e l}}=-\int_{m} \frac{\partial^{2} \tilde{\mathbf{r}}}{\partial t^{2}} d m
\end{gathered}
$$

with $\tilde{\mathbf{r}}$ being the location of a mass element with respect to the vehicle's CoM [m]. Coriolis and relative forces were defined for rocket propelled vehicles in Section 4.5.2.

The external forces produce a change in position that can be determined as follows:

$$
\frac{d \mathbf{r}_{\mathbf{c m}}}{d t}=\mathbf{V}_{\mathbf{I}}
$$

As mentioned before, describing the vehicle's motion with respect to $\mathscr{F}_{I}$ is not convenient in case of control system design and stability and controllability analyses. In this case, it is preferable to express the equations of motion with respect to $\mathscr{F}_{R}$. These can be derived from Eq.(4.30), considering that $\mathscr{F}_{R}$ is rotating with respect to $\mathscr{F}_{I}$ with an angular velocity equal to the Earth's angular velocity $\omega_{\boldsymbol{R}}=\left(0,0, \omega_{c b}\right)^{T}$. The general formulation of the equations of motion in $\mathscr{F}_{R}$ is:

$$
\mathbf{F}_{\mathbf{R}}=m \frac{\mathrm{d}^{2} \mathbf{r}_{\mathbf{c m}}}{\mathrm{d} t^{2}}+2 m \boldsymbol{\omega}_{\mathbf{R}} \times \mathbf{V}_{\mathbf{R}}+m \boldsymbol{\omega}_{\mathbf{R}} \times\left(\boldsymbol{\omega} \times \mathbf{r}_{\mathbf{c m}}\right)
$$

where $\mathbf{F}_{\mathbf{R}}$ is the sum of the external forces $[\mathrm{N}]$ acting on the vehicle (expressed with respect to $\mathscr{F}_{R}$ ), $\frac{\mathrm{d}^{2} \mathbf{r}_{\mathbf{c m}}}{\mathrm{d} t^{2}}$ is the acceleration of the vehicle $\left[\mathrm{m} / \mathrm{s}^{2}\right]$ with respect to $\mathscr{F}_{R}, 2 \boldsymbol{\omega}_{\mathbf{R}} \times \mathbf{V}_{\mathbf{R}}$ is the apparent Coriolis acceleration due to the rotation of the frame $\left[\mathrm{m} / \mathrm{s}^{2}\right]$, and $\boldsymbol{\omega}_{\boldsymbol{R}} \times\left(\boldsymbol{\omega} \times \mathbf{r}_{\mathbf{c m}}\right)$ is the apparent transport acceleration of the vehicle due to the angular rotation of the frame $\left[\mathrm{m} / \mathrm{s}^{2}\right]$.

The corresponding kinematic equation is:

$$
\frac{d \mathbf{r}_{\mathbf{c m}}}{d t}=\mathbf{V}_{\mathbf{R}}
$$

\section{EoM in Cartesian Components}

The translational dynamic EoM and kinetic equation can be written in Cartesian components in $\mathscr{F}_{I}$ as:

$$
\begin{aligned}
\frac{\mathrm{d}^{2} \mathbf{r}}{\mathrm{d} t^{2}} & =\ddot{\mathbf{r}}=(\ddot{x} \ddot{y} \ddot{z})^{T}=\frac{\mathbf{F}_{\mathbf{e}}}{m} \\
\frac{\mathrm{d} \mathbf{r}}{\mathrm{d} t} & =\dot{\mathbf{r}}=\mathbf{v}=(\dot{x} \dot{y} \dot{z})^{T}
\end{aligned}
$$

where $\mathbf{F}_{\mathbf{e}}$ indicates the external forces. As discussed before, the Cartesian components are chosen for the simulation since they can be stably and efficiently integrated numerically, and they are singularity-free. However, for conducting the analyses, Cartesian components in $\mathscr{F}_{I}$ are not easy to interpret. Thus, it is convenient to use express the vehicle's motion in spherical components with respect to $\mathscr{F}_{R}$, as presented in the next section.

\section{EoM in Spherical Components}

Mooij [1994] offers the full set of equations of motion of the translational motion, for a vehicle of variable mass $m$ subjected to external forces of aerodynamic, propulsion and gravitational origin, written in spherical coordinates in $\mathscr{F}_{R}$, i.e.:

$$
\begin{gathered}
\dot{V}_{g}=\frac{F_{V}}{m}+\omega_{c b}^{2} R \cos \delta\left(\sin \gamma_{g} \cos \delta-\cos \gamma_{g} \sin \delta \cos \chi_{g}\right) \\
V_{g} \dot{\gamma_{g}}=\frac{F_{\gamma}}{m}+2 \omega_{c b} V_{g} \cos \delta \sin \chi_{g}+\frac{V_{g}^{2}}{R} \cos \gamma_{g}+\omega_{c b}^{2} R \cos \delta\left(\cos \gamma_{g} \cos \delta+\sin \gamma_{g} \sin \delta \cos \chi_{g}\right)
\end{gathered}
$$




$$
\begin{aligned}
V_{g} \cos \gamma_{g} \dot{\chi_{g}}= & \frac{F_{\chi}}{m}+2 \omega_{c b} V_{g}\left(\sin \delta \cos \gamma_{g}-\cos \delta \sin \gamma_{g} \cos \chi_{g}\right)+\frac{V_{g}^{2}}{R} \cos ^{2} \gamma_{g} \tan \delta \sin \chi_{g}+ \\
& +\omega_{c b}^{2} R \cos \delta \sin \delta \sin \chi_{g}
\end{aligned}
$$

where $F_{V}, F_{\gamma}$ and $F_{\chi}$ are defined as follows:

$$
\begin{aligned}
F_{V}= & -D+T\left(\cos \alpha_{g} \cos \beta_{g} \cos \psi_{T} \cos \epsilon_{T}+\sin \beta_{g} \sin \psi_{T} \cos \epsilon_{T}-\sin \alpha_{g} \cos \beta_{g} \sin \epsilon_{T}\right)-m g \sin \gamma_{g} \\
F_{\gamma}= & -\left[S+T\left(\cos \alpha_{g} \sin \beta_{g} \cos \psi_{T} \cos \epsilon_{T}+\cos \beta_{g} \sin \psi_{T} \cos \epsilon_{T}-\sin \alpha_{g} \sin \beta_{g} \sin \epsilon_{T}\right)\right] \sin \sigma_{g}+ \\
& +\left[L+T\left(\sin \alpha_{g} \cos \psi_{T} \cos \epsilon_{T}+\cos \alpha_{g} \sin \epsilon_{T}\right)\right] \cos \sigma_{g}-m g \cos \gamma_{g} \\
F_{\chi}= & -\left[S+T\left(\cos \alpha_{g} \sin \beta_{g} \cos \psi_{T} \cos \epsilon_{T}+\cos \beta_{g} \sin \psi_{T} \cos \epsilon_{T}-\sin \alpha_{g} \sin \beta_{g} \sin \epsilon_{T}\right)\right] \cos \sigma_{g}+ \\
& -\left[L+T\left(\sin \alpha_{g} \cos \psi_{T} \cos \epsilon_{T}+\cos \alpha_{g} \sin \epsilon_{T}\right)\right] \sin \sigma_{g}
\end{aligned}
$$

where $D, S$, and $L$ are aerodynamic forces, i.e., drag force, side force, and lift force, respectively, $T$ is the magnitude of the thrust force, $\epsilon_{T}$ and $\psi_{T}$ are the elevation angle and the azimuth defining the direction of the thrust vector with respect to $\mathscr{F}_{B}$. Finally, $g$ is the radial gravitational acceleration that is defined in Section 3.1.

The corresponding kinematic equations are:

$$
\begin{gathered}
\dot{R}=\dot{h}=V_{g} \sin \gamma_{g} \\
\dot{\tau}=\frac{V_{g} \sin \chi_{g} \cos \gamma_{g}}{R \cos \delta} \\
\dot{\delta}=\frac{V_{g} \cos \chi_{g} \cos \gamma_{g}}{R}
\end{gathered}
$$

Note that the subscript "g" in $V_{g}, \gamma_{g}, \chi_{g}, \alpha_{g}, \beta_{g}, \sigma_{g}$ indicates that they are groundspeed-based. Also, some singularities occur in these equations of motion for $V=0 \mathrm{~m} / \mathrm{s}$, from Eq. (4.39), and for $\gamma= \pm 90^{\circ}$, from Eq. (4.40). From the kinetic equations a singularity for latitude $\delta= \pm 90^{\circ}$ (i.e., north and south pole) exists. However, these conditions are not encountered in our flight conditions.

\subsubsection{Equations of the Rotational Motion}

The rotational motion is defined as follows:

$$
\tilde{\mathbf{M}}_{\mathbf{c m}}=\mathbf{M}_{\mathbf{c m}}+\mathbf{M}_{\mathbf{C}}+\mathbf{M}_{\mathbf{r e l}}=\int_{m} \tilde{\mathbf{r}} \times\left(\frac{d \boldsymbol{\omega}}{d t} \times \tilde{\mathbf{r}}\right) d m+\int_{m} \tilde{\mathbf{r}} \times[\boldsymbol{\omega} \times(\boldsymbol{\omega} \times \tilde{\mathbf{r}})] d m
$$

where $\tilde{\mathbf{M}}_{\mathbf{c m}}$ is the pseudo external moment about the vehicle's CoM [Nm], $\mathbf{M}_{\mathbf{c m}}$ is the sum of the external moments about the vehicle's CoM $[\mathrm{Nm}], \mathbf{M}_{\mathbf{C}}$ is the Coriolis moment $[\mathrm{Nm}]$, and $\mathbf{M}_{\text {rel }}$ is the relative moment [Nm]. $\mathbf{M}_{\mathbf{C}}$ and $\mathbf{M}_{\mathbf{r e l}}$ are due to the variation of mass distribution with time and they are equal to:

$$
\begin{gathered}
\mathbf{M}_{\mathbf{C}}=-2 \int_{m} \tilde{\mathbf{r}} \times\left(\boldsymbol{\omega} \times \frac{\partial \tilde{\mathbf{r}}}{\partial t}\right) \mathrm{d} m \\
\mathbf{M}_{\text {rel }}=-\int_{m} \tilde{\mathbf{r}} \times \frac{\partial^{2} \tilde{\mathbf{r}}}{\partial t^{2}} \mathrm{~d} m
\end{gathered}
$$

which are defined for rocket propelled vehicles in Section 4.5.2. Finally, the first term of the right-hand side of Eq. (4.47) is the apparent moment due to the angular acceleration of the body with respect to the $\mathscr{F}_{I}$ and the second term is the apparent moment due to the angular velocity of the body with respect to $\mathscr{F}_{I}$.

The kinematic equation describes the change in attitude generated by the external moments as follows:

$$
\dot{\mathbf{q}}=\frac{1}{2} \Xi(\mathbf{q}) \omega
$$

where $\mathbf{q}$ is the quaternion describing the vehicle's attitude and $\Xi$ is defined to be the matrix relating the rotation vector of $\mathscr{F}_{B}$ with respect to $\mathscr{F}_{I}$, to the time derivative of the quaternion (see Eqs. (4.6) and (4.7) for the definition). 


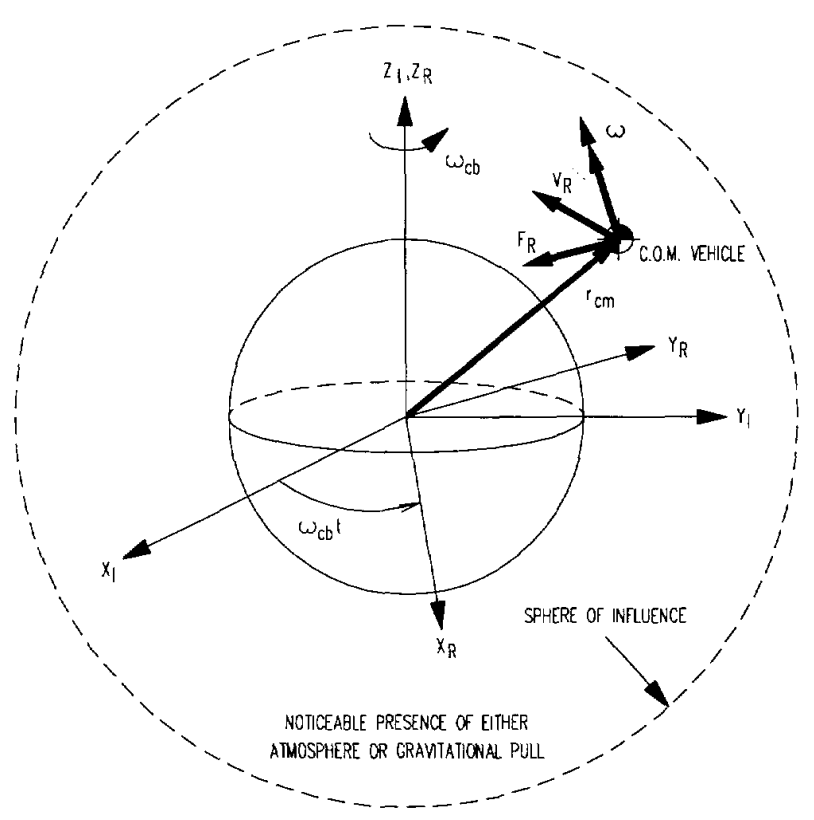

Figure 4.8: Motion of the vehicle about the Earth. Subscripts "I" and "R" indicate $\mathscr{F}_{I}$ and $\mathscr{F}_{R}$, respectively [Mooij, 1998a].

\section{Euler Equations}

If one solves the integrals of Eq.(4.47), the equations of the rotational motion can be expressed by evaluating the components along the body axes. The resulting set of non-linear equations defines the Euler equations. This set describes the rotational motion of the vehicle with respect to $\mathscr{F}_{B}$.

Let us consider a vehicle moving with respect to $\mathscr{F}_{R}$ as shown in Figure 4.8. The vehicle has variable mass $m$, located at distance $\mathbf{r}_{\mathbf{c m}}$ form the Earth's CoM. The body is moving with a velocity $\mathbf{V}_{\mathbf{R}}$ with respect to $\mathscr{F}_{R}$, with an angular velocity $\boldsymbol{\omega}$ with respect to $\mathscr{F}_{I}$, and it is subjected to the external forces $\mathbf{F}_{\mathbf{R}}$ and moments $\tilde{\mathbf{M}}_{\mathbf{c m}}$. The rotational motion of this vehicle can be described through the Euler equations, defined as:

$$
\dot{\omega}=\mathbf{I}^{-1}\left(\tilde{\mathbf{M}}_{\mathbf{c m}}-\omega \times \mathbf{I} \boldsymbol{\omega}\right)
$$

with

$$
\mathbf{I}=\left[\begin{array}{ccc}
I_{x x} & -I_{x y} & -I_{x z} \\
-I_{y x} & I_{y y} & -I_{y z} \\
-I_{z x} & -I_{z y} & I_{z z}
\end{array}\right]
$$

being a symmetric matrix that contains the moments and the products of inertia of the vehicle. The sum of the external, Coriolis and relative moments about the CoM is represented by $\tilde{\mathbf{M}}_{\mathbf{c m}}=\left[M_{x}, M_{y}, M_{z}\right]^{T}$, and $\boldsymbol{\omega}=$ $(p, q, r)^{T}$ is the angular rotation vector of $\mathscr{F}_{B}$ with respect to $\mathscr{F}_{I}$. The components of both vectors are directed along the body axes. All components of these variables are expressed along the body axes. The Euler equations can be written showing the explicit dependency of $\dot{p}, \dot{q}, \dot{r}$ on $p, q, r$ and the inertia moments and products. These expressions have been derived by Duke et al. [1988].

Although it is not a problem to solve the complete set of differential equations with a complete inertia matrix in numerical application, simplified equations can be useful in case of analytical analyses. Assuming that the body is rotational symmetric in mass, the Euler equations can be simplified because the products of inertia are zero, i.e., $\mathbf{I}=\operatorname{diag}\left[\begin{array}{lll}I_{x x} & I_{y y} & I_{z z}\end{array}\right]$. This assumption is valid for our reference vehicle since the products of inertia are much smaller that the moments of inertia (see Table 2.5). So, the rotational EoM become:

$$
\begin{aligned}
& \dot{p}=\frac{M_{x}}{I_{x x}}+\frac{I_{y y}-I_{z z}}{I_{x x}} q r \\
& \dot{q}=\frac{M_{y}}{I_{y y}}+\frac{I_{z z}-I_{x x}}{I_{y y}} p r \\
& \dot{r}=\frac{M_{z}}{I_{z z}}+\frac{I_{x x}-I_{y y}}{I_{z z}} q p
\end{aligned}
$$




\section{Kinetic EoM in Aerodynamic Angles}

The kinematic equations can be formulated using the aerodynamic angles:

$$
\begin{aligned}
& \dot{\alpha}_{g} \cos \beta_{g}=-p \cos \alpha_{g} \sin \beta_{g}+q \cos \beta_{g}-r \sin \alpha_{g} \sin \beta_{g}+ \\
& +\sin \sigma_{g}\left[\dot{\chi}_{g} \cos \gamma_{g}-\dot{\delta} \sin \chi_{g} \sin \gamma_{g}+\left(\dot{\tau}+\omega_{c b}\right)\left(\cos \delta \cos \chi_{g} \sin \gamma_{g}-\sin \delta \cos \gamma_{g}\right)\right]+ \\
& -\cos \sigma_{g}\left[\dot{\gamma_{g}}-\dot{\delta} \cos \chi_{g}-\left(\dot{\tau}+\omega_{c b}\right) \cos \delta \sin \chi_{g}\right] \\
& \dot{\beta_{g}}=p \sin \alpha_{g}-r \cos \alpha_{g}+\sin \sigma_{g}\left[\dot{\gamma_{g}}-\dot{\delta} \cos \chi_{g}-\left(\dot{\tau}+\omega_{c b}\right) \cos \delta \sin \chi_{g}\right]+ \\
& +\cos \sigma_{g}\left[\dot{\chi_{g}} \cos \gamma_{g}-\dot{\delta} \sin \chi_{g} \sin \gamma_{g}+\left(\dot{\tau}+\omega_{c b}\right)\left(\cos \delta \cos \chi_{g} \sin \gamma_{g}-\sin \delta \cos \gamma_{g}\right)\right] \\
& \dot{\sigma}_{g}=-p \cos \alpha_{g} \cos \beta_{g}-q \sin \beta_{g}-r \sin \alpha_{g} \cos \beta_{g}+\dot{\alpha}_{g} \sin \beta_{g}-\dot{\chi_{g}} \sin \gamma_{g}-\dot{\delta} \sin \chi_{g} \cos \gamma_{g}+ \\
& +\left(\dot{\tau}+\omega_{c b}\right)\left(\cos \delta \cos \chi_{g} \cos \gamma_{g}-\sin \delta \sin \gamma_{g}\right)
\end{aligned}
$$

where the aerodynamic angles $\left(\alpha_{g}, \beta_{g}\right.$, and $\left.\sigma_{g}\right)$ and speed parameters $\left(\gamma_{g}\right.$ and $\left.\chi_{g}\right)$ are groundspeed based.

In summary, the Eqs.(4.36) and (4.37) define the translational motion in Cartesian coordinates in $\mathscr{F}_{I}$. For the rotational motion, the Euler equation is used (Eq.(4.51)), describing the orientation (and its evolution in time) of a vehicle with respect to $\mathscr{F}_{I}$. The kinetic equation for the rotational motion is provided by Eq.(4.50). These equations are convenient for the simulation of the vehicle's motion. The Eqs. from (4.38) to (4.46) describe the translational motion in spherical components with respect to $\mathscr{F}_{R}$, and Eqs. from (4.53) to (4.58) describe the rotational motion in aerodynamic angles and roll, pitch, yaw rate with respect to $\mathscr{F}_{B}$. These equations are essential for the linearisation and the definition of the state-space model, which is necessary for control system design and stability and controllability analyses. The state-space model is provided in Section 4.7.

\subsubsection{Wind Equations}

The EoM presented in this chapter determine the trajectory of the vehicle with respect to the Earth. In presence of wind, the airspeed changes in amplitude and direction differing from the groundspeed. As a consequence, the aerodynamic forces and moments acting on the body change and also the trajectory with respect to the reference frame differs. It follows that it is fundamental to take into account the presence of wind introducing it in the EoM. The theory provided below comes from Mooij [1994].

The vehicle's velocity with respect to the Earth's surface can be defined as the vectorial summation of the vehicle's velocity with respect to air and air velocity with respect to the ground, i.e.:

$$
\mathbf{V}_{\text {vehicle/ground }}=\mathbf{V}_{\text {vehicle/air }}+\mathbf{V}_{\text {air/ground }}
$$

The presence of wind influences the vehicle's motion by modifying the aerodynamic forces and moments, which are strongly dependent on the airflow parameters. So, once the wind is considered in the aerodynamic model, the aerodynamic forces need to be transformed to $\mathscr{F}_{A G}$ to be introduced in the EoM. From the previous expression, if the groundspeed and wind velocity are known in spherical coordinates, the airspeed can be computed with respect to $\mathscr{F}_{V}$ as follows:

$$
\begin{gathered}
V_{a}=\sqrt{V_{g}^{2}+V_{w}^{2}-2 V_{g} V_{w}\left(\cos \gamma_{g} \cos \gamma_{w} \cos \left(\chi_{w}-\chi_{g}\right)+\sin \gamma_{g} \sin \gamma_{w}\right)} \\
\gamma_{a}=\operatorname{asin}\left(\frac{V_{g} \sin \gamma_{g}-V_{w} \sin \gamma_{w}}{V_{a}}\right) \\
\chi_{a}=\operatorname{atan}\left(\frac{V_{g} \sin \chi_{g} \cos \gamma_{g}-V_{w} \sin \chi_{w} \cos \gamma_{w}}{V_{g} \cos \chi_{g} \cos \gamma_{g}-V_{w} \cos \chi_{w} \cos \gamma_{w}}\right)
\end{gathered}
$$

where $V_{a}>0 \mathrm{~m} / \mathrm{s}$ and, from the second equation, a singularity exists for $\gamma= \pm 90^{\circ}$. Here, $V_{g}, \gamma_{g}$ and $\chi_{g}$ are the groundspeed parameters, while $V_{w}, \gamma_{w}$ and $\chi_{w}$ represent the wind parameters.

The airspeed-based aerodynamic angles can be calculated by using the groundspeed-based aerodynamic angles that come from the equations of rotational motion and the airspeed parameters defined above. The transformation matrix from $\mathscr{F}_{V}$ to $\mathscr{F}_{B}$ is defined as:

$$
\mathbf{C}_{\mathbf{B}, \mathbf{V}}=\mathbf{C}_{\mathbf{y}}\left(\alpha_{g}\right) \mathbf{C}_{\mathbf{z}}\left(\beta_{g}\right) \mathbf{C}_{\mathbf{x}}\left(-\sigma_{g}\right) \mathbf{C}_{\mathbf{y}}\left(\gamma_{g}\right) \mathbf{C}_{\mathbf{z}}\left(\chi_{g}\right)
$$

which can also be expressed as:

$$
\mathbf{C}_{\mathbf{B}, \mathbf{V}}=\mathbf{C}_{\mathbf{B}, \mathbf{T A}} \mathbf{C}_{\mathbf{T A}, \mathbf{V}} \quad \text { with } \mathbf{C}_{\mathbf{B}, \mathbf{T A}}=\mathbf{C}_{\mathbf{y}}\left(\alpha_{a}\right) \mathbf{C}_{\mathbf{z}}\left(-\beta_{a}\right) \mathbf{C}_{\mathbf{x}}\left(-\sigma_{a}\right)
$$


from which, since $\mathbf{C}_{\mathbf{V}, \mathbf{T A}}=\mathbf{C}_{\mathbf{z}}\left(-\chi_{a}\right) \mathbf{C}_{\mathbf{y}}\left(-\gamma_{a}\right)$ is known, we can invert the expression and solve for $\mathbf{C}_{\mathbf{B}, \mathbf{T A}}=\mathbf{C}_{\mathbf{B}, \mathbf{V}} \mathbf{C}_{\mathbf{V}, \mathbf{T A}}$. It yields:

$$
\mathbf{C}_{\mathbf{B}, \mathbf{T A}}=\left[\begin{array}{ccc}
\cos \alpha_{a} \cos \beta_{a} & -\cos \alpha_{a} \sin \beta_{a} \cos \sigma_{a}+\sin \alpha_{a} \sin \sigma_{a} & \cos \alpha_{a} \sin \beta_{a} \sin \sigma_{a}+\sin \alpha_{a} \cos \sigma_{a} \\
\sin \beta_{a} & \cos \beta_{a} \cos \sigma_{a} & -\cos \beta_{a} \sin \sigma_{a} \\
\sin \alpha_{a} \cos \beta_{a} & -\sin \alpha_{a} \sin \beta_{a} \cos \sigma_{a}+\cos \alpha_{a} \sin \sigma_{a} & \sin \alpha_{a} \sin \beta_{a} \sin \sigma_{a}+\cos \alpha_{a} \cos \sigma_{a}
\end{array}\right]
$$

from which the airspeed-based aerodynamic angles can be computed:

$$
\alpha_{a}=\operatorname{atan}\left(\frac{\mathbf{C}_{\mathbf{B}, \mathbf{T A}}(3,1)}{\mathbf{C}_{\mathbf{B}, \mathbf{T A}}(1,1)}\right), \quad \beta_{a}=\operatorname{asin}\left(\mathbf{C}_{\mathbf{B}, \mathbf{T A}}(2,1)\right), \quad \sigma_{a}=\operatorname{atan}\left(-\frac{\mathbf{C}_{\mathbf{B}, \mathbf{T A}}(2,3)}{\mathbf{C}_{\mathbf{B}, \mathbf{T A}}(2,2)}\right)
$$

with $\alpha_{a}=\left[-\frac{\pi}{2}, \frac{\pi}{2}\right], \beta_{a}=[-\pi, \pi], \sigma_{a}=[-\pi, \pi]$.

At this point, the aerodynamic forces and moments can be calculated on the basis of $\alpha_{a}, \beta_{a}, M$ and $q_{d y n}$ (based on $V_{a}$ ). So, the resulting forces are defined with respect to $\mathscr{F}_{A A}$. However, in the EoM they need to be expressed in $\mathscr{F}_{A G}$, so the following transformation is required:

$$
\mathbf{F}_{\mathbf{a}, \mathbf{A G}}=\mathbf{C}_{\mathbf{A G}, \mathbf{A A}} \mathbf{F}_{\mathbf{a}, \mathbf{A A}} \quad \text { with } \mathbf{C}_{\mathbf{A G}, \mathbf{A A}}=\mathbf{C}_{\mathbf{A G}, \mathbf{T G}} \mathbf{C}_{\mathbf{T G}, \mathbf{A A}}
$$

which can be easily determined on the basis of the transformation matrix definition given in Section 4.4.

\subsection{State-Space Form}

The stability of a vehicle can be analysed by studying the equations of its motion. The set of non-linear EoM defined in the previous sections are adequate to simulate the vehicle's motion, but not the most appropriate set to analyse the stability and controllability characteristics. This is due to their dependency on time, which holds back the nature of the motion modes, and to their non-linearities, which make the system behaviour dependent on magnitude of inputs and, thus, prevent from examining the vehicle's eigenmotion. Indeed, the characteristic motion of a vehicle can be defined by the eigenvalues and eigenvectors of the EoM that can be determined when the time derivatives of the states are formulated as a linear combination of the states. Thus, to the study the open-loop behaviour of a vehicle, the EoM have to be linearised. Also, the nominal trajectory need to be discretised into a number of time intervals so that, for each of the time interval, the state and control variables and the other parameters of the system are constant.

By linearising and discretising the equations of motion the overall system is transformed into a sequence of so-called Linear Time-Invariant (LTI) systems. The linearised EoM describe the variation of the state variables as function of the parameters that influence the systems, i.e., state variables and control variables. These equations are generally written in matrix form, which is known as the state-space form equations. The information provided in the following sections are gathered from Mooij [1998b], if not otherwise specified.

To linearise the EoM, some assumptions are necessary to simplify the system:

- the oblateness and the rotation of the Earth has a second-order effect, thus we can assume that the Earth is spherical and non-rotating $\left(\omega_{c b}=0 \mathrm{rad} / \mathrm{s}\right)$;

- the vehicle can be considered to be rotational symmetric (in mass) and, consequently, all the products of inertia are zero, i.e., $I_{x y}=I_{y z}=I_{x z}=0$;

- the vehicle's attitude does not depend on the asymmetrical translational motion, which means that the trajectory is parallel to the equator.

The first assumption can be justified if we consider that the rotational rate of the Earth is much smaller compared to the rotational rate of the vehicle. The second assumption is generally verified, since the products of inertia are much smaller than the moments of inertia, as can be seen in Table 2.5. The last assumption is generally valid since the attitude dynamics is much faster than translational dynamics. With these assumptions the equations for $\chi, \tau$, and $\delta$ are decoupled from the other equations, so the linearised EoM in state-space form can be defined by 9 equations for $V, \gamma, R, p, q, r, \alpha, \beta, \sigma$.

From the linearisation procedure of the non-linear EoM, for which the interested reader is referred to [Mooij, 2015], a set of differential equations is defined. This set can be written in state-space form, which is advantageous for the stability and controllability analyses since it allows to examine the system response to perturbations in any of the state and control variables. The set of linearised equations of motion in matrix form can be formulated as follows:

$$
\Delta \dot{\mathbf{x}}=\mathbf{A} \Delta \mathbf{x}+\mathbf{B} \Delta \mathbf{u}
$$


where $\Delta \mathbf{x}$ is the state deviation vector with dimensions $n \times 1, \Delta \mathbf{u}$ is the control deviation vector with dimensions $m \times 1, \mathbf{A}$ is the state coefficient matrix with dimensions $n \times n$, and $\mathbf{B}$ is the control coefficient matrix with dimensions $n \times m$. The entries of the $\mathbf{A}$ and $\mathbf{B}$ matrices correspond to the (constant) coefficients that multiply the state and control deviations, respectively, which appear in the linearised EoM. In the following of this work, the $\Delta$ of $\Delta \mathbf{x}$ and $\Delta \mathbf{u}$ will be dropped for the sake of convenience, but we should note that for the rest of the discussion $\mathbf{x}$ and $\mathbf{u}$ represent the deviation of state and control variables and not the state and control variables themselves. As specified in Section 4.1, three models of linearised EoM in state-space form are required to achieve the thesis objectives: one describing the translational and rotational motion (9 states) of the open-loop system, and two describing the rotational motion of the closed-loop system. The latter two models differentiate for the control variables used. One, needed to design the controller of the reference vehicle, has the momentfractions as control variables. The other one, needed for the verification of the control system, has the controlsurface deflections and thruster moments as control variables.

\subsubsection{Open-loop Model}

The analysis of the eigenmotion of a vehicle requires the study of the homogeneous part of the state-space model:

$$
\dot{\mathbf{x}}=\mathbf{A x}
$$

where the state variables are:

$$
\mathbf{x}=\left(\begin{array}{lllllllll}
\Delta V & \Delta \gamma & \Delta R & \Delta p & \Delta q & \Delta r & \Delta \alpha & \Delta \beta & \Delta \sigma
\end{array}\right)^{T}
$$

and, for the open-loop system, the deviations of the control-variables from their nominal values are set to zero.

To study the open-loop behaviour of the vehicle the state-space model of the full set of linear differential equations is defined as follows:

$$
\left(\begin{array}{c}
\Delta \dot{V} \\
\Delta \dot{\gamma} \\
\Delta \dot{R} \\
\Delta \dot{p} \\
\Delta \dot{q} \\
\Delta \dot{r} \\
\Delta \dot{\alpha} \\
\Delta \dot{\beta} \\
\Delta \dot{\sigma}
\end{array}\right)=\left[\begin{array}{ccccccccc}
a_{V V} & a_{V \gamma} & a_{V R} & 0 & 0 & 0 & a_{V \alpha} & 0 & 0 \\
a_{\gamma V} & a_{\gamma \gamma} & a_{\gamma R} & 0 & 0 & 0 & a_{\gamma \alpha} & a_{\gamma \beta} & a_{\gamma \sigma} \\
a_{R V} & a_{R \gamma} & 0 & 0 & 0 & 0 & 0 & 0 & 0 \\
0 & 0 & 0 & 0 & 0 & 0 & 0 & a_{p \beta} & 0 \\
a_{q V} & 0 & 0 & 0 & 0 & 0 & a_{q \alpha} & 0 & 0 \\
0 & 0 & 0 & 0 & 0 & 0 & 0 & a_{r \beta} & 0 \\
a_{\alpha V} & a_{\alpha \gamma} & a_{\alpha R} & 0 & a_{\alpha q} & 0 & a_{\alpha \alpha} & 0 & a_{\alpha \sigma} \\
a_{\beta V} & a_{\beta \gamma} & a_{\beta R} & a_{\beta p} & 0 & a_{\beta r} & 0 & a_{\beta \beta} & a_{\beta \sigma} \\
a_{\sigma V} & a_{\sigma \gamma} & 0 & a_{\sigma p} & 0 & a_{\sigma r} & a_{\sigma \alpha} & a_{\sigma \beta} & a_{\sigma \sigma}
\end{array}\right]\left(\begin{array}{c}
\Delta V \\
\Delta \gamma \\
\Delta R \\
\Delta p \\
\Delta q \\
\Delta r \\
\Delta \alpha \\
\Delta \beta \\
\Delta \sigma
\end{array}\right)
$$

where elements $a_{i j}$ of matrix A are specified in Section A.1 of Appendix A.

\subsubsection{Closed-Loop Rotational Model}

For control design purposes, the state-space model describing only the rotational motion is needed. Thus, the state variables become:

$$
\mathbf{x}=\left(\begin{array}{llllll}
\Delta p & \Delta q & \Delta r & \Delta \alpha & \Delta \beta & \Delta \sigma
\end{array}\right)^{T}
$$

Similarly, the control variables can be identified. Given the variety of actuators that can be used in ascent and re-entry trajectory dependently on the dynamic pressure, it is convenient to have control variables that are independent of which actuators are active. In this way, the same model can be used for all the different portion of flight. The moment fractions, which are defined as the fraction of the maximum available control moments, can be used. Thereafter, the required moment fractions are allocated to the active actuators. The control variables can be defined as:

$$
\mathbf{u}=\left(\begin{array}{lll}
\eta_{x} & \eta_{y} & \eta_{z}
\end{array}\right)^{T}
$$

The state-space model, which characterises the rotational motion of the closed-loop system, is defined as follows:

$$
\left(\begin{array}{c}
\Delta \dot{p} \\
\Delta \dot{q} \\
\Delta \dot{r} \\
\Delta \dot{\alpha} \\
\Delta \dot{\beta} \\
\Delta \dot{\sigma}
\end{array}\right)=\left[\begin{array}{cccccc}
0 & 0 & 0 & 0 & a_{p \beta} & 0 \\
0 & 0 & 0 & a_{q \alpha} & 0 & 0 \\
0 & 0 & 0 & 0 & a_{r \beta} & 0 \\
0 & a_{\alpha q} & 0 & a_{\alpha \alpha} & 0 & a_{\alpha \sigma} \\
a_{\beta p} & 0 & a_{\beta r} & 0 & a_{\beta \beta} & a_{\beta \sigma} \\
a_{\sigma p} & 0 & a_{\sigma r} & a_{\sigma \alpha} & a_{\sigma \beta} & a_{\sigma \sigma}
\end{array}\right]\left(\begin{array}{c}
\Delta p \\
\Delta q \\
\Delta r \\
\Delta \alpha \\
\Delta \beta \\
\Delta \sigma
\end{array}\right)+\left[\begin{array}{ccc}
b_{p \eta_{x}} & 0 & 0 \\
0 & b_{q \eta_{y}} & 0 \\
0 & 0 & b_{r \eta_{z}} \\
0 & 0 & 0 \\
0 & 0 & 0 \\
0 & 0 & 0
\end{array}\right]\left(\begin{array}{c}
\eta_{x} \\
\eta_{y} \\
\eta_{z}
\end{array}\right)
$$


with elements of matrix A, i.e., $a_{i j}$, and matrix B, i.e., $b_{i j}$, specified in Section A.2 of Appendix A.

To verify the control system, we need to perform an acceptance test (see Section B.2) by comparing the performance of the implemented control system with available reference data (e.g., [Brinkman, 2017], [Rijnsdorp, 2017], [Mooij, 1998a]). These references used the state-space model with the control-surface deflections and thruster moments as control variables to design the controller for HORUS re-entry flight. This is the reason why we need such a model for the control-system verification. This verified control system will then function as benchmark to verify the controller designed for the reference vehicle. Along the re-entry trajectory, HORUS can be controlled by means of the elevation, aileron, rudder, and the thruster moments in $x, y, z$ directions. Thus, for this model the state variables remain the same, while the control variables are:

$$
\mathbf{u}=\left(\begin{array}{llllll}
\Delta \delta_{e} & \Delta \delta_{a} & \Delta \delta_{r} & \Delta M_{T, x} & \Delta M_{T, y} & \Delta M_{T, z}
\end{array}\right)^{T}
$$

The state-space model, which characterises the rotational motion of the closed-loop system, is defined as follows:

$$
\begin{aligned}
\left(\begin{array}{c}
\Delta \dot{p} \\
\Delta \dot{q} \\
\Delta \dot{r} \\
\Delta \dot{\alpha} \\
\Delta \dot{\beta} \\
\Delta \dot{\sigma}
\end{array}\right] & =\left[\begin{array}{cccccc}
0 & 0 & 0 & 0 & a_{p \beta} & 0 \\
0 & 0 & 0 & a_{q \alpha} & 0 & 0 \\
0 & 0 & 0 & 0 & a_{r \beta} & 0 \\
0 & a_{\alpha q} & 0 & a_{\alpha \alpha} & 0 & a_{\alpha \sigma} \\
a_{\beta p} & 0 & a_{\beta r} & 0 & a_{\beta \beta} & a_{\beta \sigma} \\
a_{\sigma p} & 0 & a_{\sigma r} & a_{\sigma \alpha} & a_{\sigma \beta} & a_{\sigma \sigma}
\end{array}\right]\left(\begin{array}{c}
\Delta p \\
\Delta q \\
\Delta r \\
\Delta \alpha \\
\Delta \beta \\
\Delta \sigma
\end{array}\right)+ \\
& +\left[\begin{array}{cccccc}
0 & b_{p a} & 0 & b_{p x} & 0 & 0 \\
b_{q e} & 0 & 0 & 0 & b_{q y} & 0 \\
0 & b_{r a} & b_{r r} & 0 & 0 & b_{r z} \\
0 & 0 & 0 & 0 & 0 & 0 \\
0 & 0 & 0 & 0 & 0 & 0 \\
0 & 0 & 0 & 0 & 0 & 0
\end{array}\right]\left(\begin{array}{c}
\Delta \delta_{e} \\
\Delta \delta_{a} \\
\Delta \delta_{a} \\
\Delta M_{T, x} \\
\Delta M_{T, y} \\
\Delta M_{T, z}
\end{array}\right)
\end{aligned}
$$

with elements of matrix A, i.e., $a_{i j}$, and matrix B, i.e., $b_{i j}$, specified in Section A.2 of Appendix A. 


\section{5}

\section{Stability \& Flying Qualities}

The requirements for aircraft's flying qualities are specified in MIL-F-8785C [1980] and MIL-HDBK-1797 [1997]. These requirements are defined to ensure that aircraft are designed with the following capabilities [Roskam, 2001]:

- maintaining a steady-state flight throughout the flight envelope

- manoeuvring safely from one steady-state flight condition to another

- being trimmable in certain flight conditions

- keeping the control forces within specified limits throughout the design envelope.

Mathematical models that are based on the equations of motion, aerodynamic and thrust forces and moments, can be employed to predict the compliance of a vehicle to these regulations. The EoM in state-space form, which are provided in Section 4.7, enable the analysis of the eigenmotion of the system, whose methodology is described in Section 5.1. Section 5.1.1 illustrates the stability problems that can be encountered in hypersonic flight and the differences with respect to subsonic flight. Section 5.2 offers the military specifications that are defined mainly for subsonic aircraft. Section 5.3 introduces the method for quality assurance. Finally, the studied and designed tools are used to analyse the stability and flying qualities: Section 5.4 studies the stability characteristics, determining the eigenmotion of the hypersonic vehicle and the trim conditions so that the flying qualities of the open-loop system can be analysed. The compliance of hypersonic vehicles to the MIL-specifications is investigated.

\subsection{Eigenmotion}

When the motion of a body is under the effect of perturbations, we are generally interested in studying the dynamic stability and the response of the system to these perturbations. Roskam [2001] defines the $d y$ namic stability as the behaviour of a perturbed body to return to the initial equilibrium conditions or to a new steady-state condition after the perturbation has stopped. If the body establishes a motion of oscillatory or pure convergence about the equilibrium point it is said to be dynamically stable, while, if its motion about the equilibrium point is an oscillatory or pure divergence, then it is said to be dynamically unstable. Finally, a body is said to be neutrally stable, if it establishes an oscillatory motion about the equilibrium point with constant amplitude. A disturbance could be internally or externally generated: for example, an internal disturbance is a control surface deflection commanded by the pilot and an external disturbance is a variation of angle of attack due to turbulences or wind gusts. The response behaviour of an aircraft to a disturbance can be predicted in advance using the system of linearised EoM written in state-space form.

The analysis the eigenmotion, also known as open-loop behaviour or free response (i.e., no control is applied) of the system, corresponds to the study of the system's response to small perturbations of the state variables. Thus, the only homogeneous part of the state-space form equation, i.e., $\dot{\mathbf{x}}=\mathbf{A x}$, is needed. The eigenmotion of the system is defined once the eigenvalues $\lambda$ and eigenvectors $\boldsymbol{\mu}$ of the state matrix $\mathbf{A}$ are known, as follows [Kuo, 1987]:

$$
\mathbf{x}_{\lambda}(t)=e^{\lambda t} \boldsymbol{\mu}
$$


and for a full set of linear equations, we get:

$$
\mathbf{x}_{\lambda}(t)=\sum_{i=1}^{n} c_{i} e^{\lambda t} \boldsymbol{\mu}
$$

with $c_{i}$ being a constant defined from the initial conditions $\mathbf{x}(0)$, and $n$ the number of coupled equations that compose the linear system. As mentioned before, all the state variables need to be characterised to completely study the characteristic motion of FSS-1 along its trajectory, thus the full set of equations of translational and rotational motion is used, i.e., $n=9$. The state-space model for the open-loop system is specified in Section 4.7.1. In a software environment, like Matlab ${ }^{\circledR}$, standard algorithms are available to compute the eigenvalues and eigenvectors of a matrix [Press et al., 2007].

The stability and periodicity of the motion can be examined by studing the eigenvalues. If an eigenvalue is a real number then the characteristic motion is aperiodic, while if it is complex the eigenmotion is periodic. It should be noticed that the complex eigenvalues always come in conjugate pairs. The stability of the motion depends on the sign of the real part of the eigenvalues: if it is zero, the eigenmotion presents a constant amplitude; if it is negative, the eigenmotion is converging (stable); if it is positive, the eigenmotion is diverging (unstable).

Some coefficients need to be specified to characterize completely the motion of the system. For a converging motion, the time required to halve the amplitude of the deviation is called halving time, $T_{\frac{1}{2}}$, and it is defined as:

$$
T_{\frac{1}{2}}=\frac{\ln \frac{1}{2}}{\operatorname{Re}(\lambda)}
$$

It assumes negative values for a diverging motion. In this case, the doubling time $T_{2}$, which is the opposite of the halving time, is used.

For a periodic motion, the period $P$, the damping ratio $\zeta$, and the natural frequency $\omega_{n}$ are defined, respectively, as:

$$
\begin{gathered}
P=\frac{2 \pi}{\operatorname{Im}(\lambda)} \\
\omega_{n}=\sqrt{\operatorname{Re}(\lambda)^{2}+\operatorname{Im}(\lambda)^{2}} \\
\zeta=-\frac{\operatorname{Re}(\lambda)}{\omega_{n}}=-\frac{\operatorname{Re}(\lambda)}{\sqrt{\operatorname{Re}(\lambda)^{2}+\operatorname{Im}(\lambda)^{2}}}
\end{gathered}
$$

where the damping ratio is positive for converging motion, while it is negative for diverging motion. In the latter case, it could be called amplification ratio.

The study of the eigenvectors offers a deeper insight in the characteristics of the eigenmotion. From the analysis of eigenvectors, it is possible to identify the components of the eigenmotion, that are known as modes of motion. In particular, the modulus and the argument of a eigenvector serve to comprehend, respectively, which of the state variables is involved in the motion and the phase difference between the involved state variables. Mathematically, the modulus $x$ and the argument $\theta$ of a complex number $x$ can be computed as follows:

$$
\begin{gathered}
z=\sqrt{\operatorname{Re}(x)^{2}+\operatorname{Im}(x)^{2}} \\
\theta=\operatorname{atan}\left(\frac{\operatorname{Im}(x)}{\operatorname{Re}(x)}\right)
\end{gathered}
$$

It should be noticed that the argument of a real number is 0 if $x \geq 0$ or $\pi$ if $x<0$.

In the preceding of this thesis report all the tools necessary to study the dynamic behaviour of a flight vehicle are provided. These tools can be now used to characterise its stability when small disturbances occur during the steady flight. From the study of the modes of motion that characterise the behaviour of a vehicle in response to this small perturbations are identified. The modes of motion of a spaceplane in hypersonic flight are similar to those of subsonic aircraft, presented by Etkin and Reid [1995], with some modifications due to the differences of hypersonic and subsonic regimes. For a subsonic aircraft, the modes of motion are five. They can be distinguished in longitudinal and lateral-directional modes. The longitudinal modes are the shortperiod and phugoid mode. The lateral modes are the lateral oscillation (or Dutch roll), the rolling convergence and the spiral mode. For aircraft in subsonic regime, longitudinal and lateral modes are usually decoupled. In the following, a general description of each mode of motion will be provided: 




Figure 5.1: The short-period oscillation: how it appears to an external observer [Roskam, 2001].



Figure 5.2: The phugoid: how it appears to an external observer [Roskam, 2001].

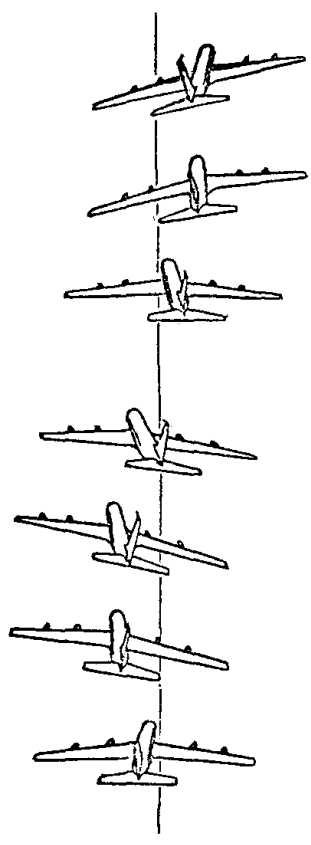

Figure 5.3: The lateral oscillation: how it appears to an external observer [Roskam, 2001]. 
Short-period oscillation: fast, periodic, usually well-damped aircraft motion. The response to a disturbance will be a fast variation of pitch angle and angle of attack with negligible speed variation. The induced aerodynamic forces are quite large. Figure 5.1 shows the short-period oscillation as seen by an external observer.

Phugoid: slow, periodic, poorly-damped eigenmotion. As can be seen in Figure 5.2, the phugoid is a continuous translation of the aircraft plane of symmetric, with negligible pitch moment. Thus, the phugoid is characterised by a continuous exchange of kinetic and potential energy, since it is involves a continuous (slow) variation of velocity and altitude with almost negligible angle-of-attack variation. These slow oscillations induce small variation in dynamic pressure and aerodynamic forces. Despite the weak damping, the period is usually so long that, in presence of a control system, it can promptly correct for this motion.

Lateral oscillation: fast, periodic, well-damped (sometimes moderately damped) eigenmotion. As shown by Figure 5.3, the main characteristic of this motion is a rotation about the yaw axis. Also, the roll angle varies continuously, while velocity and flight-path angle remain unchanged. Large aerodynamic forces act on the vehicle during this motion.

Roll convergence: aperiodic, strongly damped eigenmotion. The vehicle starts rotating about the roll-axis (Figure 5.4). A damping moment proportional to the roll rate is generated to eliminate the induced roll rate.

Spiral mode: aperiodic eigenmotion that can be either stable or unstable. It consists mainly of a rotation about the yaw-axis, with some roll and generally zero sideslip. Figure 5.5 offers a representation of the spiral mode as seen by an external observer.

The main differences in stability and control characteristics of hypersonic vehicles with respect to subsonic vehicles are presented in the next section.

\subsubsection{Stability Problems in Hypersonic Flight}

Lifting vehicles at hypersonic speed present unique stability and control characteristics that differ from those of aircraft in conventional flight regimes. These differences are mainly due to high kinetic energy level, specific properties of aerothermodynamic forces and moments, and close interaction between vehicle dynamics and engines at hypersonic speed. The effects of dynamics, engine, and control characteristics on the performance of a hypersonic vehicle have been studied by Sachs [1993]. The results of this study are presented in the following of this section.

The hypersonic flight is characterised, in addition to the short-period mode and the phugoid mode, by an other longitudinal mode of motion, which is named as height mode. The short-period mode defines the shortterm dynamics, while the phugoid and height modes define the long-term dynamics, which present different features with respect to the subsonic regime. The short-period oscillation is influenced by the same stability derivatives and inertia quantities in both the conventional and hypersonic speed regimes. However, some differences are present: differently from the subsonic regime, the velocity does not remain constant and the oscillation are much faster and hardly-damped because of the lower magnitude of the aerodynamic forces in the upper layers of the atmosphere. The phugoid in the hypersonic regime is generally an unstable motion, even if the doubling time is quite large. The oscillations generate mainly altitude changes and small (often negligible) speed variations. The altitude variations cause variations in density and, consequently, in lift force.

The height mode, which is negligible in the conventional regimes, becomes relevant from roughly Mach 3 (i.e., high-supersonic regime and higher). It is an aperiodic lightly-damped mode of motion that was discovered by Sachs [1993]. It induces drag and thrust changes by means of variations in speed and altitude. It should be noticed that, differently from the height mode, the properties of the phugoid mode are mainly influenced by altitude variations.

For hypersonic vehicles, the stability of the phugoid and height modes is affected by the pitching moments due to speed and altitude disturbances. For instance, an altitude-dependent pitching moment introduces an aperiodic instability, driving the phugoid to an aperiodic eigenmotion. Differently, a speed-dependent pitching moment has a more moderate effect on the phugoid and affects mainly the height mode. Moreover, to illustrate the differences in response to the control inputs between subsonic and hypersonic regimes, Figure 5.6 shows the response of the system to a step pitch-control deflection and to a step thrust-control input. As can be seen, the response in subsonic flight is the oscillatory motion of the phugoid, whereas in hypersonic flight the response is generally dominated by height-mode characteristics and the phugoid gives only small contribution (almost negligible in case of the step thrust control input). 


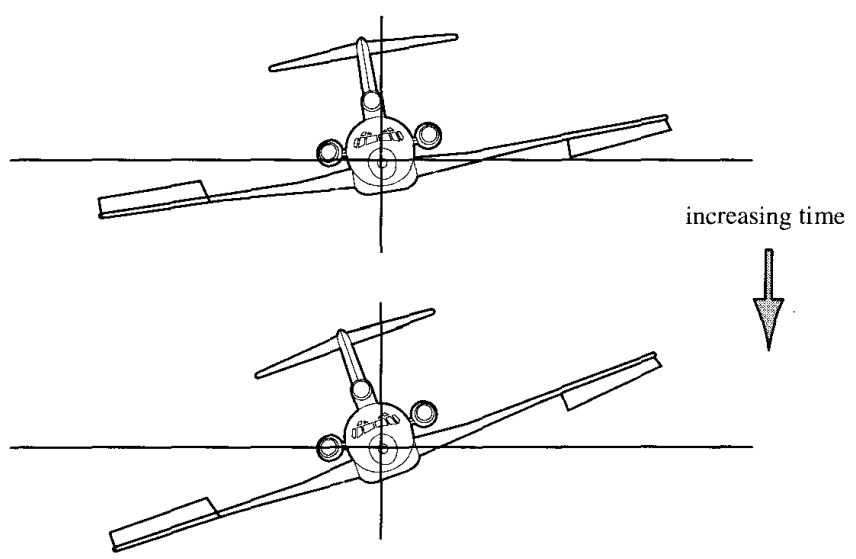

Figure 5.4: The roll convergence: how it appears to an external observer [Roskam, 2001].

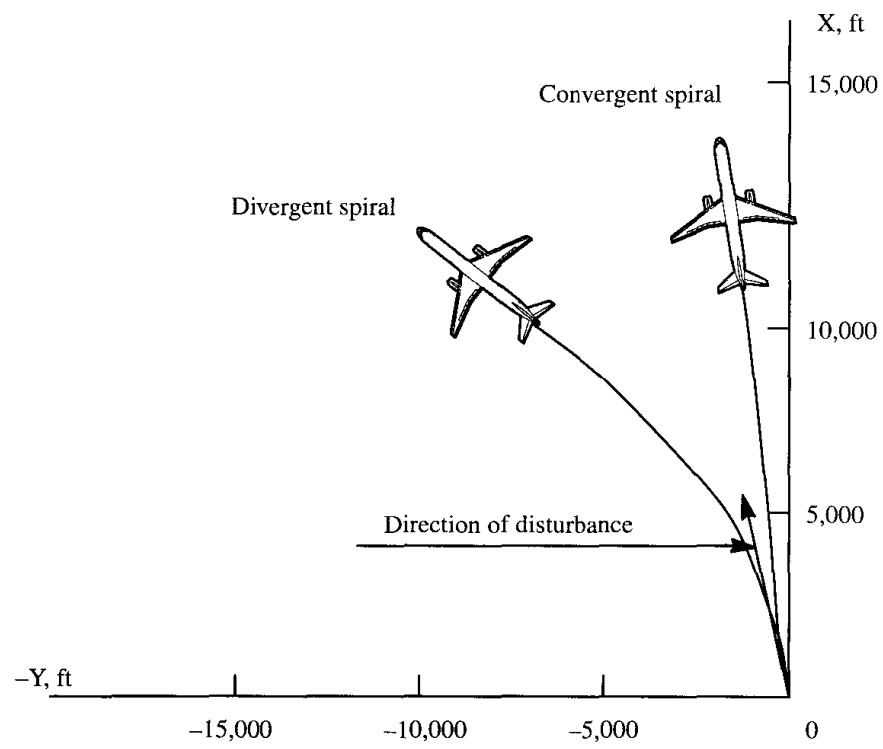

Figure 5.5: The spiral mode: how it appears to an external observer [Roskam, 2001].

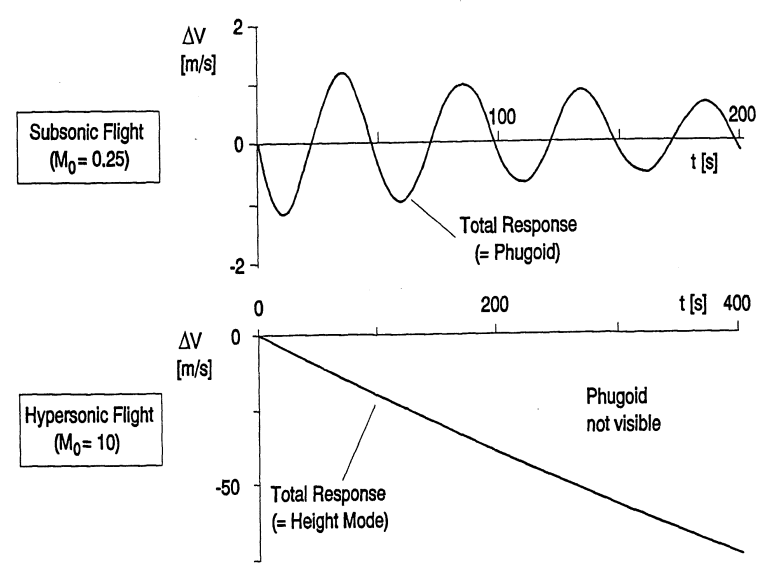

Figure 5.6: The response of the system to a step pitch control deflection (left) and to a step thrust control input (right): differences between subsonic and hypersonic flights Sachs [1993]. 
Also the lateral modes of motion present some differences in the hypersonic regime. The lateral oscillation has similar characteristics to those in subsonic regime. The only difference is that initially it can be unstable. It can be experienced as a periodic or aperiodic mode. Roll convergence and spiral mode are two aperiodic eigenmotion that are generally stable and whose main component is the bank angle. It was found that, at larger speed regime, the influence of the angle of sideslip can be larger with respect to subsonic flight.

This study was confirmed and extended by Mooij [2013], who analysed the characteristic motion of HORUS along its re-entry trajectory, as mentioned in the mission heritage (Section 1.1). The eigenmotions are compared with those of the conventional, subsonic aircraft, so that similarities and differences can be identified. It resulted that the modes of motion are generally slower due to the absence of the atmosphere in the early re-entry phase. Also, coupling between symmetric and asymmetric motion are identified. This investigation is used in the following as benchmark to verify the methodology of the eigenmotion determination, as provided in Section B.2.1, which will be employed for the stability and flying qualities analyses.

\subsection{Military Specifications}

MIL-F-8785C [1980] represents the first document that collects the flying-qualities requirements for U.S. military, manned, piloted (subsonic) aircraft. Later, this document was adapted and extended after the advances in aircraft technology. Thus, the final version MIL-HDBK-1797 [1997] was published. The MIL-Spec requirements are considered as a starting point for identifying flying qualities and controllability characteristics of the new generation of air and space means of transport, spaceplanes. In the MIL specifications the flying qualities requirements change depending on the aircraft's type and the flight phase's category. Aircraft are classified in four groups depending on size, weight and manoeuvrability. Group III of large, heavy, lowto-medium manoeuvrability aircraft presents characteristics that are similar to those of vehicles flying in the high-supersonic and hypersonic regime. So, the flying characteristics of Group III aircraft can be adopted.

The flight phases are combined into three main categories, depending on the speed of the manoeuvre, the precision tracking and the precise flight-path control required. In agreement with mission requirement $M-1$, which states that the flying qualities and controllability characteristics shall be studied in ascent and reentry, we are mainly interested in flight phases of Category B, i.e., "non-terminal flight phases that are normally accomplished using gradual manoeuvres and without precision tracking, although accurate flight-path control may be required" [MIL-HDBK-1797, 1997].

The aircraft manufacturer shall identify and specify the features, such as position of centre of mass, weight, moments and products of inertia, and thrust setting, related to aircraft normal state for each considered flight phases. Normal state is the aircraft state without system and/or component failure. Similarly, the designer is required to define the aircraft's features related to its failure states, operational flight envelopes and service flight envelopes. The MIL-documentation provides the definition for these concepts. The failure states "consist of Aircraft Normal States modified by one or more malfunctions in aircraft components or systems". The operational flight envelopes "define the boundaries in terms of speed, altitude and load factor within which the aircraft must be capable of operating in order to accomplish its missions and in which Level 1 flying qualities are required". The service flight envelopes "define the boundaries in terms of speed, altitude, and normal acceleration derived from aircraft limits as distinguished from mission requirements. [...] The boundaries of the Service Flight Envelopes can be coincident with or lie outside the corresponding operational boundaries".

The levels of flying qualities were presented in Section 1.1.1, but are reported here for the sake of convenience [MIL-HDBK-1797, 1997]:

- Level 1: "Flying qualities clearly adequate for the mission flight phase";

- Level 2: "Flying qualities adequate to accomplish the mission flight phase, but some increase in pilot workload or degradation in mission effectiveness, or both, exists";

- Level 3: "Flying qualities such that the aircraft can be controlled safely, but pilot workload is excessive or mission effectiveness is inadequate, or both". However, Category B flight phases can be completed.

Aircraft must be designed to satisfy the Level 1 requirements when all the systems are normally operating. The probability of failure of the systems is directly related to the probability of degradation of the flying qualities levels. Indeed, the MIL-Specs establish that the probability of encountering Level 2 and Level 3 after failure should be less than $10^{-2}$ and $10^{-4}$, respectively, in the operational flight envelope, while the probability of encountering Level 3 after failure should be less than $10^{-2}$ in the service flight envelope. When the aircraft meets the flying qualities requirements, the aircraft response to control inputs is supposed to be so that the 
Table 5.1: Phugoid damping requirements [MIL-HDBK-1797, 1997].

\begin{tabular}{||cc||}
\hline \multicolumn{2}{|c||}{ Phugoid } \\
\hline Level 1 & $\zeta_{p h} \geq 0.04$ \\
Level 2 & $\zeta_{p h} \geq 0$ \\
Level 3 & $T_{2_{p h}} \geq 55 \mathrm{~s}$ \\
\hline
\end{tabular}

Table 5.2: Short period damping ratio limits [MIL-HDBK-1797, 1997].

\begin{tabular}{||lc||}
\hline \multicolumn{1}{|c||}{ Short Period } \\
\hline Level 1 & $0.30 \leq \zeta_{s p} \leq 2.00$ \\
Level 2 & $0.20 \leq \zeta_{s p} \leq 2.00$ \\
Level 3 & $0.15^{\star} \leq \zeta_{s p}$ \\
\hline$\star$ This limit can be reduced for altitude higher than $6.1 \mathrm{~km}(20000 \mathrm{ft})$. \\
\hline
\end{tabular}

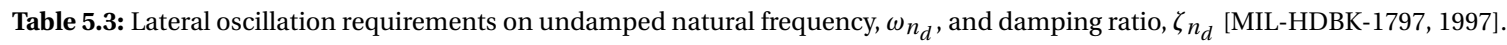

\begin{tabular}{||lccc||}
\hline \multicolumn{4}{|c||}{ Lateral Oscillation } \\
\hline Level 1 & Min. $\zeta_{n_{d}}$ & Min. $\zeta_{n_{d}} \omega_{n_{d}}[\mathrm{rad} / \mathrm{s}]$ & Min. $\omega_{n_{d}}[\mathrm{rad} / \mathrm{s}]$ \\
Level 2 & 0.08 & 0.15 & $0.4^{\star}$ \\
Level 3 & 0.02 & 0.05 & $0.4^{\star}$ \\
\hline$\star$ This requirement may be not valid for Class III vehicles. & $0.4^{\star}$ \\
\hline
\end{tabular}

mission objective can be achieved with guaranteed safety throughout the whole flight envelope. The system should provide a suitable control effort to stabilise and control the vehicle.

In the following paragraphs the longitudinal and lateral-directional flying-qualities requirements are introduced. Only those that are interesting for our study purposes are examined, i.e., the requirements dedicated to Class III aircraft in Category B flight phase. Thus, to complete the picture, the interested reader is referred to the military specifications MIL-F-8785C [1980] and MIL-HDBK-1797 [1997].

\subsubsection{Longitudinal Requirements}

MIL-F-8785C [1980] and MIL-HDBK-1797 [1997] present the longitudinal flying quality requirements. As mentioned before, the phugoid is a long-period airspeed oscillation that is experienced when a stabilized airspeed is sought after a disturbance. Requirements for the phugoid damping ratio or for the time-to-double the amplitude are presented in Table 5.1. Considering the characteristics of the phugoid presented in Section 5.1 and that it is an oscillatory phenomenon, the time-to-halve the amplitude is an important performance parameter that is defined as:

$$
T_{1 / 2_{p h}}=\frac{\ln \frac{1}{2}}{-\zeta_{p h} \omega_{n_{p h}}}
$$

Obviously, when the damping ratio is negative, the eigenmotion is amplified and Eq.(5.9) represents the timeto-double the amplitude.

Flying quality requirements for the short-period mode of motion are defined in terms of the damping ratio $\zeta_{s p}$, as presented by Table 5.2.

\subsubsection{Lateral-Directional Requirements}

MIL-F-8785C [1980] and MIL-HDBK-1797 [1997] specify the lateral-directional flying quality requirements. The requirements for the lateral oscillation are expressed in term of the undamped natural frequency, $\omega_{n_{d}}$, the damping ratio, $\zeta_{n_{d}}$, and their product. These specifications are indicated in Table 5.3.

The flying-quality criterion is given for the time required by the vehicle to perform a bank angle manoeuvre after a control input, which is known as roll control effectiveness. The requirement is met if the time to realise a 
Table 5.4: Roll control effectiveness criterion [Chalk, 1980].

\begin{tabular}{||cc||}
\hline \multicolumn{2}{|c|}{ Roll Mode } \\
\hline \multicolumn{1}{|c|}{ Cat. B } \\
Level 1 & $t_{30} \leq 4.0 \mathrm{~s}$ \\
Level 2 & $t_{30} \leq 5.0 \mathrm{~s}$ \\
Level 3 & $t_{30} \leq 6.0 \mathrm{~s}$ \\
\hline
\end{tabular}

Table 5.5: Minimum required flying qualities level in case of atmospheric disturbances [MIL-HDBK-1797, 1997].

\begin{tabular}{||ccc||}
\hline Atmospheric disturbances & $\begin{array}{c}\text { Within operational } \\
\text { flight envelope }\end{array}$ & $\begin{array}{c}\text { Within service } \\
\text { flight envelope }\end{array}$ \\
\hline Calm to light & Level 1 & Level 2 \\
Light to moderate & Level 1 & Level 2 \\
Moderate to severe & Controllable or better & Recoverable or better \\
\hline
\end{tabular}

bank-angle command is kept within certain boundaries, which are modified for high-supersonic (and higherspeed regimes) with respect to the military specifications on the basis of the results obtained by the Concorde flight tests [Chalk, 1980]. These limits are reported in Table 5.4 as the time $t_{30}$ required to realise a $30^{\circ}$ bankangle command. The roll control effectiveness requirement is linked to the controllability of the system, which will be treated in Section 7.3.

The spiral mode is not experienced in the design points of FSS-1 trajectory where the stability analyses are conducted (see Section 5.4). So, it is not further studied.

\subsubsection{Atmospheric Disturbances}

MIL-F-8785C [1980] and MIL-HDBK-1797 [1997] define the flying-qualities requirements in case that the vehicle is subjected to atmospheric adversities. The main atmospheric perturbations are the steady-state wind, wind gusts and turbulence, whose models were detailed in Section 3.3. These wind components have to be implemented in the EoM through the aerodynamic terms as presented in Section 4.6.3.

Table 5.5 shows the required minimum levels of flying qualities in presence of atmospheric disturbances. Note that for atmospheric disturbances, whose intensity is severe to moderate, a quantitative requirements has not been provided. However, qualitative requirements are given. Controllable indicates that "the aircraft can be controlled safely, but pilot workload is excessive or mission effectiveness is inadequate, or both. Category B flight phase can be completed". Recoverable means that "control can be maintained long enough to fly out of a disturbance. All flight phases can be terminated safely" [MIL-HDBK-1797, 1997].

To have a more tangible evidence that FSS-1 meets the MIL-requirements if subjected to wind along its trajectory, Daimler-Benz Aerospace [1998] expands these specifications defining that:

- in case of steady-state wind, the vehicle shall withstand steady-state wind using the $75 \%$ of the available control authority;

- in case of wind gusts, the vehicle shall withstand wind gusts with maximum amplitude of $12 \mathrm{~m} / \mathrm{s}$;

- in case of turbulence, the vehicle shall withstand turbulence modelled on the basis of the Dryden spectral model, without using the maximum allowable rates and/or deflections of the available actuators.

\subsection{Quality Assurance}

The compliance of the vehicle with the flying quality requirements can be assessed by simulation, flight tests, or both. In this thesis work, the author is capable of analysing the flying qualities of the vehicle by simulation, since it is not possible to do any flight test due to the unavailability of the vehicle and to the high cost involved.

For the present application, the compliance to the flying-quality requirements is assured by identifying the vehicle's characteristics through the state-space equation and by comparing the obtained values with the 
specified requirements. This will be analysed in Section 5.4, where the eigenmotion, trim capabilities and the flying qualities are analysed at different representative flight conditions and configurations $(M=5,10,15,20$ along ascent and re-entry trajectory).

If the requirements are not met, there is the need of providing the vehicle with a guidance, navigation and control (GNC) system that is capable of guiding and controlling the vehicle to achieve the desired performance. If, even in presence of a GNC system, the vehicle results to be unstable and uncontrollable, it can be concluded that the vehicle capabilities and characteristics need to be modified.

It should be remarked that the flying quality requirements presented in Section 5.2 are specific for manned piloted aircraft that fly at subsonic speeds. Thus, it is expected that the analysis of the flying qualities of our hypersonic vehicle could provide results that will overshoot some of the requirements. For instance, Chalk [1980] testifies that the values of response times specified in the military requirements are underestimated for high-supersonic and hypersonic vehicles, given the much slower response times at high-speed regimes.

\subsection{Stability \& Flying-Quality Analyses}

In this section the stability characteristics of the FSS-1 hypersonic flight are studied at the design points along its ascent and re-entry trajectory. The flight conditions at these points are presented in Tables 2.5. In Section 5.4.1, the modes of motion of the spaceplane are identified and the eigenvalues and eigenvectors characteristics are studied, followed by the time history of these modes. The trimmability capabilities and the effect of trim on the overall stability are investigated in Section 5.4.2. Finally, Section 5.4.3 presents the flying qualities of the FSS-1 hypersonic spaceplane, indicating whether they met the military requirements presented in Section 5.2

\subsubsection{Eigenmotion of Hypersonic Vehicles}

The characteristics of motion are generally different with respect to conventional aircraft, due to a larger speed regime and the nature of the reference trajectory. As discussed before, one of the main differences is that, from the study of the eigenvectors of the A matrix of the state-space model, it is possible to trace six modes of motion instead of five, being the height mode the additional mode of motion for hypersonic vehicles. In addition, the modes of motion can present some differences with respect to subsonic aircraft. To study the eigenmotion of FSS-1, the characteristics values of motion are computed and the modes of motion are identified by analysing the eigenvalues and eigenvector. This analysis is conducted for the design points with Mach $5,10,15,20$ along the ascent and re-entry flight. The periodic modes that are identified are the phugoid mode and the short-period mode. The terms that are the driving components of the phugoid are the height and velocity, with almost negligible variation of the angle of attack that can be, consequently, considered constant. The short-period presents a large oscillation in angle of attack and pitch rate. It is different from the conventional short-period because the height and velocity might change, while they remain constant in subsonic regime. Moreover, its period is about 30 times larger, due to its larger speed regime.

All the other modes are aperiodic. Some of them presents the same characteristics of the height modes, identified by Sachs [1993] for the first time while studying stability in hypersonic flight. One is unstable and lightly amplified. The remaining two have both the height and velocity as dominating components, thus they are named as height \& velocity modes to distinguish them from the pure height mode. One height \& velocity mode is stable and the other one is unstable, presenting a rapid convergence and strong divergence, respectively. It is notable that it can happen that more than one of the same characteristic motion is present, due to a degeneration of a complex mode in two separate modes. The remaining modes are two aperiodic lateral modes and two aperiodic roll modes. The former have the sideslip angle, bank angle, and the corresponding angular rates as main components. The latter are dominating by the bank angle, as its name suggests. One of these modes presents a larger sideslip angle with respect to the other one, thus it can be compare with the roll convergence of conventional aircraft.

Tables from 5.6 to 5.9 present the mode-of-motion characteristics at the reference points, i.e., at Mach 5, $10,15,20$, during the ascent trajectory. It should be noticed that all the values lower than $10^{-8}$ are considered to be 0 since they mainly depend on the machine accuracy. The phugoid is a stable motion and remains so in time. The damping ratio increases, becoming a well-damped motion with the time to half-the-amplitude decreasing from $460 \mathrm{~s}$ to $1.7 \mathrm{~s}$. Simultaneously, the period decreases in time, going from around $1330 \mathrm{~s}$ at Mach 5 to $570 \mathrm{~s}$ at Mach 10 and arriving at $72 \mathrm{~s}$ at Mach 15. From Mach 15 to 20, the periodic phugoid mode breaks into two aperiodic modes, both stable and highly damped $\left(\mathrm{T}_{1 / 2}=0.7 \mathrm{~s}\right)$. In these two modes the influence of 
Table 5.6: Eigenvalues and eigenvector analysis: ascending flight at Mach 5, trimmed condition $\left(\delta_{b}=10^{\circ}\right)$.

\begin{tabular}{|c|c|c|c|c|c|c|c|c|c|}
\hline \multicolumn{10}{|c|}{ Mach 5} \\
\hline$\lambda_{i}$ & \multicolumn{2}{|c|}{ Phugoid } & \multicolumn{2}{|c|}{ Height \& Velocity mode } & Height mode & \multicolumn{2}{|c|}{ Aperiodic lateral mode } & \multicolumn{2}{|c|}{ Roll mode } \\
\hline $\begin{array}{l}\operatorname{Re}\left(\lambda_{i}\right) \\
\operatorname{Im}\left(\lambda_{i}\right)\end{array}$ & $\begin{array}{l}-0.1506 . \\
\pm 0.4721 .\end{array}$ & $\begin{array}{l}0^{-2} \\
0^{-2}\end{array}$ & $\begin{array}{c}-0.1826 \\
-\end{array}$ & $\begin{array}{c}0.1838 \\
-\end{array}$ & $\begin{array}{c}0.1372 \cdot 10^{-2} \\
-\end{array}$ & $\begin{array}{c}0.2670 \\
-\end{array}$ & $\begin{array}{c}-0.2685 \\
-\end{array}$ & $\begin{array}{c}0.174 \cdot 10^{-2} \\
-\end{array}$ & $\begin{array}{c}\approx 0 \\
-\end{array}$ \\
\hline $\mathrm{P}[\mathrm{s}]$ & 1330. & & - & - & - & - & - & - & - \\
\hline $\begin{array}{c}\mathrm{T}_{1 / 2}[\mathrm{~s}] \\
\mathrm{T}_{2}[\mathrm{~s}]\end{array}$ & 460.1 & & 3.79 & 3.77 & 505.02 & 2.59 & 2.57 & 398.23 & $3.8 \cdot 10^{15}$ \\
\hline$\zeta[-]$ & 0.304 & & - & - & - & - & - & - & - \\
\hline$\omega_{n}[\mathrm{rad} / \mathrm{s}]$ & 0.005 & & - & - & - & - & - & - & - \\
\hline$\overline{\overline{\mu_{i}}}$ & $\bar{z}$ z [-] & $\theta\left[^{\circ}\right]$ & $\overline{\mathrm{z}[-]}$ & $\overline{\mathrm{z}[-]}$ & Z [-] & $\overline{\mathrm{z}[-]}$ & $\mathrm{z}[-]$ & $\overline{\mathrm{z}[-]}$ & $\mathrm{z}[-]$ \\
\hline$\Delta_{V}$ & $0.1146 \cdot 10^{-2}$ & 25.23 & 0.0148 & 0.0125 & $0.8108 \cdot 10^{-3}$ & 0 & 0 & 0 & 0 \\
\hline$\Delta_{\gamma}$ & $0.5848 \cdot 10^{-6}$ & 78.74 & $0.2180 \cdot 10^{-4}$ & $0.2207 \cdot 10^{-4}$ & $0.1538 \cdot 10^{-6}$ & 0 & 0 & 0 & 0 \\
\hline$\Delta_{R}$ & 1.0000 & 180 & 0.9999 & 0.9999 & 1.0000 & 0 & 0 & 0 & 0 \\
\hline$\Delta_{p}$ & 0 & 7.92 & 0 & 0 & 0 & 0.2244 & 0.2256 & $0.1679 \cdot 10^{-3}$ & $0.5675 \cdot 10^{-4}$ \\
\hline$\Delta_{q}$ & $0.4038 \cdot 10^{-8}$ & 133.60 & $0.2409 \cdot 10^{-3}$ & $0.2332 \cdot 10^{-3}$ & 0 & 0 & 0 & 0 & 0 \\
\hline$\Delta_{r}$ & 0 & 90.88 & 0 & 0 & 0 & 0.1272 & 0.1279 & $0.9527 \cdot 10^{-3}$ & $0.8382 \cdot 10^{-3}$ \\
\hline$\Delta_{\alpha}$ & $0.8462 \cdot 10^{-6}$ & 25.20 & $0.1309 \cdot 10^{-2}$ & $0.1272 \cdot 10^{-2}$ & $0.5992 \cdot 10^{-7}$ & 0 & 0 & 0 & 0 \\
\hline$\Delta_{\beta}$ & 0 & 101.31 & 0 & 0 & 0 & 0.4179 & 0.4225 & $0.2039 \cdot 10^{-3}$ & 0 \\
\hline$\Delta_{\sigma}^{p}$ & 0 & 30.62 & 0 & 0 & 0 & 0.8711 & 0.8684 & 1.0000 & 1.0000 \\
\hline
\end{tabular}

Table 5.7: Eigenvalues and eigenvector analysis: ascending flight at Mach 10, untrimmed and trimmed conditions.

\begin{tabular}{|c|c|c|c|c|c|c|c|c|c|}
\hline \multicolumn{10}{|c|}{ Mach 10: untrimmed conditions } \\
\hline$\lambda_{i}$ & \multicolumn{2}{|c|}{ Phugoid } & \multicolumn{2}{|c|}{ Height \& Velocity mode } & Height mode & \multicolumn{2}{|c|}{ Aperiodic lateral mode } & \multicolumn{2}{|c|}{ Roll mode } \\
\hline $\operatorname{Re}\left(\lambda_{i}\right)$ & \multirow{2}{*}{\multicolumn{2}{|c|}{$\begin{array}{l}-0.3183 \cdot 10^{-2} \\
\pm 0.6485 \cdot 10^{-2}\end{array}$}} & -0.3604 & 0.3539 & $0.4137 \cdot 10^{-4}$ & 0.4978 & -0.5048 & 0.0138 & $\approx 0$ \\
\hline $\operatorname{Im}\left(\lambda_{i}\right)$ & & & - & - & - & - & - & - & - \\
\hline $\mathrm{P}[\mathrm{s}]$ & \multicolumn{2}{|c|}{975.88} & - & - & - & - & - & - & - \\
\hline $\begin{array}{c}\mathrm{T}_{1 / 2}[\mathrm{~s}] \\
\mathrm{T}_{2}[\mathrm{~s}]\end{array}$ & \multicolumn{2}{|c|}{217.76} & 1.90 & 1.96 & 1675.4 & 1.39 & 1.37 & 50.35 & $3.2 \cdot 10^{15}$ \\
\hline$\zeta[-]$ & \multicolumn{2}{|c|}{0.4432} & - & - & - & - & - & - & - \\
\hline$\omega_{n}[\mathrm{rad} / \mathrm{s}]$ & \multicolumn{2}{|c|}{0.0072} & - & - & - & - & - & - & - \\
\hline$\mu_{i}$ & $\mathrm{z}[-]$ & $\theta\left[^{\circ}\right]$ & $\mathrm{z}[-]$ & $\mathrm{z}[-]$ & $\mathrm{z}[-]$ & $\mathrm{z}[-]$ & $\mathrm{z}[-]$ & $\mathrm{z}[-]$ & $\mathrm{z}[-]$ \\
\hline$\Delta_{V}$ & 0.0139 & 130.71 & 0.0181 & 0.0432 & $0.7257 \cdot 10^{-3}$ & 0 & 0 & 0 & 0 \\
\hline$\Delta_{\gamma}$ & $0.4953 \cdot 10^{-5}$ & 90.44 & $0.5509 \cdot 10^{-3}$ & $0.5482 \cdot 10^{-3}$ & $0.9852 \cdot 10^{-6}$ & 0 & 0 & 0 & 0 \\
\hline$\Delta_{R}$ & 1.0000 & $\mathbf{0}$ & 0.9998 & 0.9991 & 1.0000 & 0 & 0 & 0 & 0 \\
\hline$\Delta_{p}$ & 0 & 4.09 & 0 & 0 & 0 & 0.3030 & 0.3082 & $0.1127 \cdot 10^{-3}$ & $0.1016 \cdot 10^{-3}$ \\
\hline$\Delta_{q}$ & $0.3379 \cdot 10^{-6}$ & 145.29 & $0.1774 \cdot 10^{-2}$ & $0.1727 \cdot 10^{-2}$ & 0 & 0 & 0 & 0 & 0 \\
\hline$\Delta_{r}$ & 0 & 145.49 & 0 & 0 & 0 & 0.3251 & 0.3307 & $0.1136 \cdot 10^{-3}$ & $0.1026 \cdot 10^{-3}$ \\
\hline$\Delta_{\alpha}$ & $0.6667 \cdot 10^{-5}$ & 49.13 & $0.4994 \cdot 10^{-2}$ & $0.4754 \cdot 10^{-2}$ & $0.3473 \cdot 10^{-7}$ & 0 & 0 & 0 & 0 \\
\hline$\Delta_{\beta}$ & 0 & 17.08 & 0 & 0 & 0 & 0.5967 & 0.6153 & $0.6916 \cdot 10^{-3}$ & 0 \\
\hline$\Delta_{\sigma}$ & 0 & 6.79 & 0 & 0 & 0 & 0.6682 & 0.6458 & 1.0000 & 1.0000 \\
\hline \multicolumn{10}{|c|}{ Mach10: trimmed conditions: $\epsilon_{T}=0.1^{\circ}$} \\
\hline$\lambda_{i}$ & \multicolumn{2}{|c|}{ Phugoid } & \multicolumn{2}{|c|}{ Height \& Velocity mode } & Height mode & \multicolumn{2}{|c|}{ Aperiodic lateral mode } & \multicolumn{2}{|c|}{ Roll mode } \\
\hline $\operatorname{Re}\left(\lambda_{i}\right)$ & \multirow{2}{*}{\multicolumn{2}{|c|}{$\begin{array}{l}-0.3456 \cdot 10^{-2} \\
\pm 0.1138 \cdot 10^{-2}\end{array}$}} & -0.4193 & 0.4100 & $0.2234 \cdot 10^{-3}$ & 0.4978 & -0.5048 & 0.0138 & $\approx 0$ \\
\hline $\operatorname{Im}\left(\lambda_{i}\right)$ & & & - & - & - & - & - & - & - \\
\hline $\mathrm{P}[\mathrm{s}]$ & \multicolumn{2}{|c|}{569.24} & - & - & - & - & - & - & - \\
\hline $\begin{array}{c}\mathrm{T}_{1 / 2}[\mathrm{~s}] \\
\mathrm{T}_{2}[\mathrm{~s}]\end{array}$ & \multicolumn{2}{|c|}{200.55} & 1.66 & 1.69 & 1303.4 & 1.39 & 1.37 & 50.40 & $1.3 \cdot 10^{15}$ \\
\hline$\zeta[-]$ & \multicolumn{2}{|c|}{0.2988} & - & - & - & - & - & - & - \\
\hline$\omega_{n}[\mathrm{rad} / \mathrm{s}]$ & \multicolumn{2}{|c|}{0.0116} & - & - & - & - & - & - & - \\
\hline$\overline{\mu_{i}}$ & $\overline{z \text { z [-] }}$ & $\theta\left[^{\circ}\right]$ & $\overline{\mathrm{z}[-]}$ & $\bar{z}$ z [-] & $\bar{z}[-]$ & $z$ z [-] & $\bar{z}[-]$ & $\overline{\mathrm{z}[-]}$ & $\bar{z}$ z [-] \\
\hline$\Delta_{V}$ & 0.0136 & 146.38 & 0.0236 & 0.0486 & $0.2906 \cdot 10^{-3}$ & 0 & 0 & 0 & 0 \\
\hline$\Delta_{\gamma}$ & $0.2026 \cdot 10^{-4}$ & 88.27 & $0.6359 \cdot 10^{-3}$ & $0.6342 \cdot 10^{-3}$ & $0.4804 \cdot 10^{-6}$ & 0 & 0 & 0 & 0 \\
\hline$\Delta_{R}$ & 1.0000 & $\mathbf{0}$ & 0.9997 & 0.9988 & 1.0000 & 0 & 0 & 0 & 0 \\
\hline$\Delta_{p}$ & 0 & 124.73 & 0 & 0 & 0 & 0.3030 & 0.3082 & $0.1127 \cdot 10^{-3}$ & $0.1001 \cdot 10^{-3}$ \\
\hline$\Delta_{q}$ & $0.3616 \cdot 10^{-6}$ & 164.59 & $0.2749 \cdot 10^{-2}$ & $0.2797 \cdot 10^{-2}$ & 0 & 0 & 0 & 0 & 0 \\
\hline$\Delta_{r}$ & 0 & 16.02 & 0 & 0 & 0 & 0.3251 & 0.3307 & $0.1136 \cdot 10^{-3}$ & $0.1026 \cdot 10^{-3}$ \\
\hline$\Delta_{\alpha}$ & $0.3541 \cdot 10^{-5}$ & 33.22 & $0.6692 \cdot 10^{-2}$ & $0.6438 \cdot 10^{-2}$ & $0.7585 \cdot 10^{-7}$ & 0 & 0 & 0 & 0 \\
\hline$\Delta_{\beta}$ & 0 & 109.44 & 0 & 0 & 0 & 0.5966 & 0.6153 & $0.6908 \cdot 10^{-3}$ & 0 \\
\hline$\Delta_{\sigma}$ & 0 & 167.42 & 0 & 0 & 0 & 0.6682 & 0.6458 & 1.0000 & 1.0000 \\
\hline
\end{tabular}


Table 5.8: Eigenvalues and eigenvector analysis: ascending flight at Mach 15, trimmed condition $\left(\epsilon_{T}=0.15^{\circ}\right)$.

\begin{tabular}{|c|c|c|c|c|c|c|c|c|c|}
\hline \multicolumn{10}{|c|}{ Mach 15} \\
\hline$\lambda_{i}$ & \multicolumn{2}{|c|}{ Phugoid } & \multicolumn{2}{|c|}{ Short period oscillation } & Height mode & \multicolumn{2}{|c|}{ Aperiodic lateral mode } & \multicolumn{2}{|c|}{ Roll mode } \\
\hline $\operatorname{Re}\left(\lambda_{i}\right)$ & \multirow{2}{*}{\multicolumn{2}{|c|}{$\begin{array}{r}-0.4078 \\
\pm 0.2258\end{array}$}} & \multirow{2}{*}{\multicolumn{2}{|c|}{$\begin{array}{l}0.2801 \\
0.0875\end{array}$}} & $0.6786 \cdot 10^{-5}$ & 0.6284 & -0.6322 & 0.0684 & $\approx 0$ \\
\hline $\operatorname{Im}\left(\lambda_{i}\right)$ & & & & & - & - & - & - & - \\
\hline $\mathrm{P}[\mathrm{s}]$ & \multicolumn{2}{|c|}{71.78} & \multicolumn{2}{|c|}{27.83} & - & - & - & - & - \\
\hline $\begin{array}{c}\mathrm{T}_{1 / 2}[\mathrm{~s}] \\
\mathrm{T}_{2}[\mathrm{~s}]\end{array}$ & \multicolumn{2}{|l|}{1.70} & \multicolumn{2}{|c|}{2.48} & 70826.3 & 1.11 & 1.09 & 10.14 & $3.1 \cdot 10^{15}$ \\
\hline$\zeta[-]$ & \multicolumn{2}{|c|}{0.9777} & \multicolumn{2}{|c|}{-0.7784} & - & - & - & - & - \\
\hline$\omega_{n}[\mathrm{rad} / \mathrm{s}]$ & \multicolumn{2}{|c|}{0.4171} & \multicolumn{2}{|c|}{0.3597} & - & - & - & - & - \\
\hline$\mu_{i}$ & $\bar{z}$ Z [-] & $\theta\left[^{\circ}\right]$ & $\bar{z}$ z [-] & $\theta\left[^{\circ}\right]$ & $\bar{z}$ z [-] & $\bar{z}$ Z [-] & $\bar{z}[-]$ & $\bar{z}$ Z [-] & $\bar{z}$ [ [-] \\
\hline$\Delta_{V}$ & 0.0769 & 161.65 & 0.0957 & 157.26 & $0.2094 \cdot 10^{-5}$ & 0 & 0 & 0 & 0 \\
\hline$\Delta_{\gamma}$ & $0.4158 \cdot 10^{-6}$ & 166.14 & $0.4064 \cdot 10^{-6}$ & 37.66 & $0.9610 \cdot 10^{-7}$ & 0 & 0 & 0 & 0 \\
\hline$\Delta_{R}$ & 1.0000 & $\mathbf{0}$ & 1.0000 & $\mathbf{0}$ & 1.0000 & 0 & 0 & 0 & 0 \\
\hline$\Delta_{p}$ & 0 & 0.46 & 0 & 26.73 & 0 & 0.27 .10 & 0.2910 & $0.3585 \cdot 10^{-2}$ & $0.8198 \cdot 10^{-2}$ \\
\hline$\Delta_{q}$ & $0.1326 \cdot 10^{-8}$ & 129.60 & $0.1543 \cdot 10^{-2}$ & 104.12 & 0 & 0 & 0 & 0 & 0 \\
\hline$\Delta_{r}$ & 0 & 34.54 & 0 & 73.93 & 0 & 0.4410 & 0.4735 & 0.0952 & 0.0902 \\
\hline$\Delta_{\alpha}$ & $0.4456 \cdot 10^{-6}$ & 28.98 & $0.3185 \cdot 10^{-2}$ & 87.61 & $0.6639 \cdot 10^{-7}$ & 0 & 0 & 0 & 0 \\
\hline$\Delta_{\beta}$ & 0 & 137.58 & 0 & 89.25 & 0 & 0.6523 & 0.7045 & 0.0153 & 0 \\
\hline$\Delta_{\sigma}$ & 0 & 0 & 0 & 156.84 & 0 & 0.5538 & 0.4413 & 1.0000 & 1.0000 \\
\hline
\end{tabular}

Table 5.9: Eigenvalues and eigenvector analysis: ascending flight at Mach 20, trimmed condition $\left(\epsilon_{T}=0.26^{\circ}\right)$.

\begin{tabular}{|c|c|c|c|c|c|c|c|c|c|}
\hline \multicolumn{10}{|c|}{ Mach 20} \\
\hline$\lambda_{i}$ & \multicolumn{2}{|c|}{ Short-period oscillation } & \multicolumn{2}{|c|}{ Pitch divergence mode } & \multirow{2}{*}{$\begin{array}{l}\text { Height mode } \\
0.2234 \cdot 10^{-5}\end{array}$} & \multicolumn{2}{|c|}{ Aperiodic lateral mode } & \multicolumn{2}{|c|}{ Roll mode } \\
\hline $\operatorname{Re}\left(\lambda_{i}\right)$ & 0.21 & & -0.9594 & -0.9043 & & 0.9151 & -0.7394 & 0.0172 & $\approx 0$ \\
\hline $\operatorname{Im}\left(\lambda_{i}\right)$ & \pm 0.26 & & - & - & - & - & - & - & - \\
\hline $\mathrm{P}[\mathrm{s}]$ & 23.6 & & - & - & - & - & - & - & - \\
\hline $\begin{array}{c}\mathrm{T}_{1 / 2}[\mathrm{~s}] \\
\mathrm{T}_{2}[\mathrm{~s}]\end{array}$ & 3.2 & & 0.65 & 0.77 & $3.10 \cdot 10^{5}$ & 0.76 & 0.94 & 40.24 & $1.7 \cdot 10^{15}$ \\
\hline$\zeta[-]$ & -0.62 & & - & - & - & - & - & - & - \\
\hline$\omega_{n}[\mathrm{rad} / \mathrm{s}]$ & 0.33 & & - & - & - & - & - & - & - \\
\hline$\overline{\mu_{i}}$ & $\bar{z}[-]$ & $\theta\left[^{\circ}\right]$ & $\bar{z}[-]$ & $\overline{z \text { z [-] }}$ & $\mathrm{z}[-]$ & $\bar{z}[-]$ & $\overline{z \text { z [-] }}$ & $\bar{z}[-]$ & $\mathrm{z}[-]$ \\
\hline$\Delta_{V}$ & 0.0502 & 137.21 & 0.9676 & 0.5859 & $0.1177 \cdot 10^{-5}$ & 0 & 0 & 0 & 0 \\
\hline$\Delta_{\gamma}$ & $0.7345 \cdot 10^{-6}$ & 51.09 & $0.1070 \cdot 10^{-4}$ & $0.1832 \cdot 10^{-4}$ & $0.5275 \cdot 10^{-6}$ & 0 & 0 & 0 & 0 \\
\hline$\Delta_{R}$ & 1.0000 & $\mathbf{0}$ & 0.9516 & 0.9099 & 1.0000 & 0 & 0 & 0 & 0 \\
\hline$\Delta_{p}$ & 0 & 177.62 & 0 & 0 & 0 & 0.0235 & 0.0270 & $0.7116 \cdot 10^{-2}$ & $0.9254 \cdot 10^{-4}$ \\
\hline$\Delta_{q}$ & $0.1535 \cdot 10^{-2}$ & 115.83 & $0.1105 \cdot 10^{-4}$ & $0.1029 \cdot 10^{-4}$ & 0 & 0 & 0 & 0 & 0 \\
\hline$\Delta_{r}$ & 0 & 151.52 & 0 & 0 & 0 & 0.5985 & 0.6867 & 0.1809 & 0.1808 \\
\hline$\Delta_{\alpha}$ & $0.1976 \cdot 10^{-2}$ & 112.91 & 0.0130 & 0.0170 & $0.4560 \cdot 10^{-7}$ & 0 & 0 & 0 & 0 \\
\hline$\Delta_{\beta}$ & 0 & 51.51 & 0 & 0 & 0 & 0.7819 & 0.7250 & 0 & 0 \\
\hline$\Delta_{\sigma}$ & 0 & 38.47 & 0 & 0 & 0 & 0.1729 & 0.0457 & 1.0000 & 1.0000 \\
\hline
\end{tabular}

the angle of attack and pitch rate becomes more important, increasing of four orders of magnitude. For this reason, they could seem to be height \& velocity modes, but the higher influence of $\Delta q$ leads to define them as pitch convergence modes.

The height \& velocity modes are two aperiodic modes, of which one is strongly damped $\left(\mathrm{T}_{1 / 2}=3.8 \mathrm{~s}\right)$ and the other one is strongly diverging $\left(\mathrm{T}_{2}=3.7 \mathrm{~s}\right)$. The times to half/double-the-amplitude decreases in time, meaning that the stable mode becomes more stable and unstable one becomes even more unstable. At Mach 15, these two aperiodic modes converge in one periodic mode: the short-period mode, which is unstable with $T_{2}$ of 2.5 $\mathrm{s}$ at $M=15$ and $3.3 \mathrm{~s} M=20$. The height mode is an aperiodic mode, which is initially unstable with $T_{2}=505 \mathrm{~s}$ at Mach 5. It becomes less unstable in time, reaching a $T_{2}$ of order $\sim 10^{5} \mathrm{~s}$ at Mach 15 and, finally, it turns into a stable mode at Mach 20.

The aperiodic lateral motion presents two modes, one stable and one unstable. From the eigenvalues, it is possible to deduce that at Mach 5 strong convergence of the stable mode and strong divergence of the unstable mode are induced. In time the stable and unstable components become more emphasised, which is a sign of the fact that the system is not tending to improve its lateral stability properties. A similar situation is experienced for the aperiodic roll motion, which is degenerated into two roll modes. One is unstable with a time to double-the-amplitude that is decreasing over time, but at Mach 20 it starts to grow. The other mode is stable, but it is characterised by a large values of $T_{2}$ of order $\sim 10^{15} \mathrm{~s}$, so it does not clearly contribute to the stability of the system.

As discussed in the preceding chapters, the reference vehicle FSS-1 was derived from the HORUS configuration, whose eigenmotion of the re-entry flight was studied by Mooij [2013]. From the stability analysis, it is 
interesting to notice that FSS-1 presents similar characteristics to the HORUS eigenmotion along the re-entry trajectory. The analysis of eigenmotion of FSS-1 along the re-entry trajectory is described in the following. The phugoid presents the same driving components, i.e., velocity and altitude, that has in the ascent case, but along re-entry this mode is unstable, although the doubling time is quite high $\left(T_{2} \sim 10^{4} \mathrm{~s}\right)$. The short period is not experienced in the design points where the eigenmotion is analysed. The height mode is a lightly damped aperiodic mode. For what concerns the rotational motion, the lateral oscillation mode is initially unstable, but later it starts to be damped to finally become stable. The aperiodic roll mode is mainly stable and aperiodic. Similarly to the ascent flight, it includes two types of modes, one of which presents a larger influence of the sideslip angle, resembling the roll convergence mode.

In particular, the time history of the eigenmotion of FSS-1 along the re-entry trajectory can be described. The eigenmotion is characterised, at the beginning, by two periodic modes, i.e., phugoid and periodic lateral oscillation. These modes are all unstable, with a time-to-double the amplitude of order $10^{4} \mathrm{~s}$ for the phugoid and between 50 and $150 \mathrm{~s}$ for the periodic lateral mode. The aperiodic mode experienced are the roll convergence and the height mode, which are both described by two modes, one unstable and one stable, the latter being the predominant one. Over time, the periodic modes become less unstable with increasing time-to-double the amplitude, but without reaching a stable condition. At Mach 5 the lateral oscillation change into an aperiodic lateral mode. It presents one stable and one unstable component, with a quite low time-to-halve/double the amplitude. This means that a strong convergence and a strong divergence are induced, respectively. A similar behaviour is reported by two components of the height \& velocity mode, but at Mach 5 they finally merge into one stable height mode with $T_{1 / 2}=2000 \mathrm{~s}$. Finally, the roll convergence continues to be represented by two modes, but at the end of the flight the unstable component turns to be the predominant one.

It can be concluded that the vehicle is not stable along both its ascent and re-entry flight. Although in the following section it is investigated how trimming the vehicle can improve stability, this analysis suggests that the vehicle needs to be controlled to have a stable flight and achieve the objectives of the mission.

\subsubsection{Trim Analysis}

To complete the stability analysis of the open-loop system, a trim analysis is conducted for the ascent and re-entry trajectory. The ascent trajectory is characterised by two main phases, which are separated by the booster burn-out at Mach 9. The trim along the ascending flight can be provided, in principle, by the body flap and TVC.

The trim law allows to define the deflection angle of the body flap and TVC elevation so that longitudinal static stability can be obtained, i.e., resulting pitch moment equal to zero. The trim analysis was conducted studying three options: trim provided by body flap only, trim provided by TVC only, and trim provided by body flap for the $50 \%$ and TVC for the remaining 50\%. At Mach 5, a body-flap deflection of $\delta_{b}=10^{\circ}$ is sufficient to trim the vehicle, although the other two cases are possible: trim provided by only TVC requires $\epsilon_{T}=0.2^{\circ}$, and the case of trim provided by both body flap and TVC results in $\delta_{b}=3.26^{\circ}$ and $\epsilon_{T}=0.1^{\circ}$. In this flight condition, using TVC for trim does not improve or change significantly the stability characteristics with respect to use partially or totally the body flap. Differently, for Mach $>5$ the effectiveness of the body flap reduces drastically with decreasing dynamic pressure, until it cannot provide any convenient contribution to trim for $\bar{q} \leq 5000$ $\mathrm{Pa}$. Thus, above Mach 5 the need for thrust vectoring arises. Thus, for all the design points characterised by $\bar{q} \leq 5000 \mathrm{~Pa}$, the vehicle is trimmed using TVC only.

A measure that is essential to quantify the stability and the controllability of aircraft is the static stability margin. It evaluates the non-dimensional distance between the CoM and the neutral point position, expressed in percentage of the reference length: $\frac{x_{N}}{L_{r e f}}=-\frac{C_{m \alpha}}{C_{L \alpha}}$. The vehicle is stable when the static margin is positive, i.e., $-\frac{C_{m \alpha}}{C_{L \alpha}}>0$. Moreover, the larger the static margin, the more stable is the vehicle [Roskam, 2001]. It is shown in Figure 5.7 that the static margin along the ascent trajectory tends to instabilities for $M \geq 5$. This can be confirmed also by analysing the aerodynamic dataset: Figure 2.3 shows that the $C_{m \alpha}$ slope is positive for hypersonic Mach numbers and the range of angle of attack that is of interest for the hypersonic ascending flight $\left(2.5^{\circ} \leq \alpha \leq 5^{\circ}\right)$. In addition, with Mach number increasing the $C_{L \alpha}$ slope reduces inducing the static margin to be amplified towards instabilities. It is highlighted that trim improves stability of the system, but it does not make the vehicle stable.

Along the re-entry trajectory the vehicle can be trimmed by using the body flap and, if it is not sufficient, the elevator. Figure 5.7 presents the variation of the static margin as function of Mach along the re-entry trajectory for both trimmed and untrimmed condition. It indicates instability along the whole re-entry flight, although the vehicle becomes less unstable after Mach 10. The difference with respect to the ascent trajectory is mainly 




Figure 5.7: Static stability margin as function of Mach number (calculated at points with $M=5,10,15,20$ ) along the ascent and re-entry.

linked to the different range of angles of attack that interests the relative portion of flight. Indeed, along the reentry the angle of attack goes from about $30^{\circ}$ at $M=20$ to $12^{\circ}$ at $M=5$, for which the $C_{m \alpha}$ slope becomes less negative. Similarly to what happens along ascent, trim makes the vehicle less unstable, but it is not sufficient to reach stability.

The influence of trim on the eigenmotion of the vehicle is detailed here just for the flight conditions at Mach 10 along the ascent trajectory to avoid a tedious repetition of long tables. However, the behaviour is quite representative of the characteristics of motion at the other design points of the trajectory. Table 5.7 reports the eigenmotion properties in case of untrimmed and trimmed conditions using TVC. The trim actually generates more significant changes in the longitudinal modes. This was expected because the trim influences mainly the longitudinal motion. The trimmed condition makes the phugoid more stable, reducing the period of about 400 s. For the remaining modes, we can see that trimming the vehicle induces the unstable modes and the stable modes to become more unstable and more stable, respectively. From analysing this table, it can be concluded that trimming the vehicle helps to reduce the instabilities of the system, especially along the longitudinal motion, but it does not suffice to make it stable on the whole, confirming that FSS-1 needs to be continuously and actively controlled along its flight, as was concluded from the analysis of the static stability margin.

\subsubsection{Flying Qualities}

The flying qualities of the FSS-1 along the ascent and reentry trajectory are analysed in this section. The FSS-1 flying qualities are studied by comparing them with the set of military specifications defined by MILF-8785C [1980] and MIL-HDBK-1797 [1997], given in Section 5.2. The requirements are given for both longitudinal and lateral modes of motion of piloted, subsonic aircraft. However, it was mentioned before that the characteristic eigenmotion of hypersonic spaceplanes shows some fundamental differences with respect to the conventional aircraft. From the investigation of the eigenmotion, it was proved that the spiral mode does not occur in the design points considered for this study along the FSS-1 trajectory. So, the spiral mode is not further studied in this section. Furthermore, the lateral mode is periodic along the re-entry trajectory and aperiodic during the ascent flight, thus the requirements on the damping ratio and natural frequency are applicable only for the re-entry.

Figures 5.8 and 5.9 illustrate the requirements of the phugoid and short-period oscillation, respectively. The characteristics of the periodic longitudinal motion along the ascent and re-entry flight are reported for the design points. It is reminded that no short-period oscillation is experienced during re-entry. The phugoid is a stable motion along ascent, achieving Level 2 at Mach 5 but reaching Level 1 from Mach 10 onwards. Indeed, it was previously noticed that it becomes more stable over time. This can be a direct consequence of trimming the vehicle. Differently, along re-entry the phugoid is characterised by negative damping ratios, being an unstable mode. It deteriorates up to Mach 15 and, finally, it improves at Mach 20 achieving Level 2. However, Level 3, which is not presented in the figure because it is given in terms of time-to-double the amplitude, is assured if $T_{2} \geq 55 \mathrm{~s}$. This condition is met in all the points along re-entry. Thus it can be concluded that the phugoid satisfies Level 1 along the ascent and Level 3 along the re-entry trajectory. The short-period oscillation is experienced only in the last portion of the ascent flight, where it does not meet the requirements, as shown in Figure 5.9. 


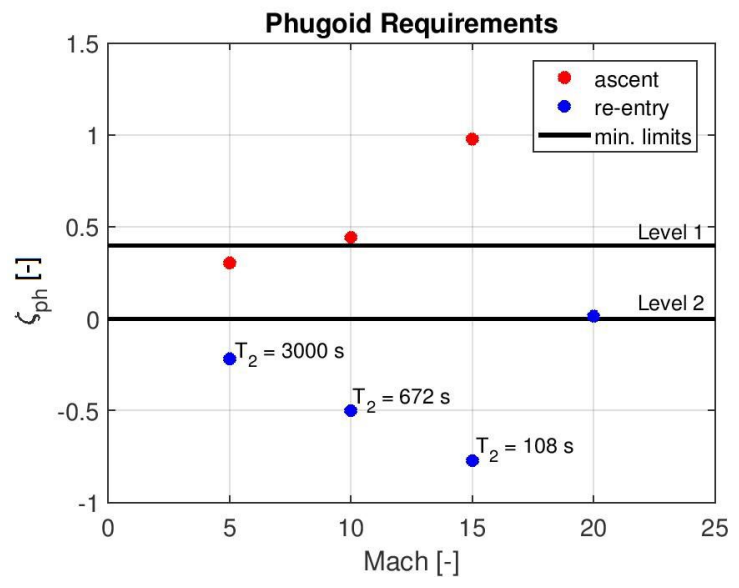

Figure 5.8: Phugoid requirements on the damping ratio $\zeta_{p h}$ [MIL-HDBK-1797, 1997] and characteristics of FSS-1 eigenmotion as function of Mach number (calculated at the points of interest with $M=5,10,15,20)$ along ascent and re-entry trajectory.

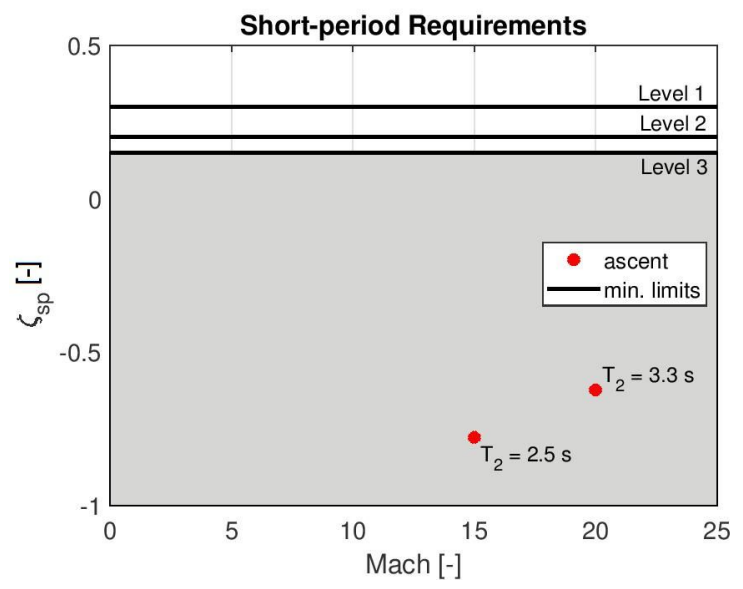

Figure 5.9: Short-period requirements on the damping ratio [MIL-HDBK-1797, 1997] and characteristics of FSS-1 eigenmotion at the design points with $M=15,20$ along ascent trajectory. The gray area represents the zone where MIL requirements are not met.

Table 5.10: Lateral oscillation mode of motion: requirements [MIL-HDBK-1797, 1997] and characteristics of FSS-1 eigenmotion.

\begin{tabular}{|c|c|c|c|}
\hline \multicolumn{4}{|c|}{ Lateral Oscillation Requirements } \\
\hline & $\zeta_{n_{d}[-]}$ & $\zeta_{n_{d}} \omega_{n_{d}}[\mathrm{rad} / \mathrm{s}]$ & $\omega_{n_{d}}[\mathrm{rad} / \mathrm{s}]$ \\
\hline Level 1 & $\zeta_{n_{d}} \geq 0.08$ & $\zeta_{n_{d}} \omega_{n_{d}} \geq 0.15$ & $\omega_{n_{d}} \geq 0.4^{\star}$ \\
\hline Level 2 & $\zeta_{n_{d}} \geq 0.02$ & $\zeta_{n_{d}} \omega_{n_{d}} \geq 0.05$ & $\omega_{n_{d}} \geq 0.4^{\star}$ \\
\hline Level 3 & $\zeta_{n_{d}} \geq 0$ & Not specified & $\omega_{n_{d}} \geq 0.4^{\star}$ \\
\hline \multicolumn{4}{|c|}{ FSS-1 Characteristics (re-entry) } \\
\hline Mach 15 & -0.06 & -0.02 & 0.36 \\
\hline Mach 20 & -0.005 & -0.007 & 1.29 \\
\hline & \multicolumn{3}{|c|}{$\begin{array}{l}\text { Requirement not met } \\
\text { Requirement met }\end{array}$} \\
\hline
\end{tabular}

The last periodic mode is the lateral mode, also known as Dutch mode. This is experienced only in the initial phase of the re-entry flight. Table 5.10 describes the requirements and the relative characteristics of the FSS-1 motion. Two over three requirements are not met, thus it is concluded that the lateral mode is not in compliance with the MIL-Specs.

The MIL-requirement about the control effectiveness is part of the controllability analysis (see Section 7.3), since they define the time required to realise a roll command. Also, the response to atmospheric disturbances is 
not studied in open-loop, since it was demonstrated through the eigenmotion, trimmability and flying-quality analyses that FSS-1 is unstable even in absence of atmospheric perturbations. So, it can be concluded that they will destabilise the vehicle even more. The response to atmospheric disturbances will be analysed for the system controlled by a robust control system (see Section 7.4).

It should be noticed that this analysis is obtained studying the behaviour of the system in some key points of the hypersonic trajectory. Thus, despite from this discussion it is possible to draw an overall picture of the spaceplane eigenmotion features, the study of the eigenmotion along the whole trajectory could be beneficial to extent this analysis and understand deeply how the eigenmotion evolves over time. Moreover, it is reminded that the requirements we used to verify the flying qualities are taken from the military specifications for conventional aircraft. Thus, it is likely that the requirements are too strict for the hypersonic flight. However, defining the exact flying qualities requirements for hypersonic vehicle goes beyond the scope of this thesis work. In fact, this analysis is appropriate to have insight into the system motion characteristics and provide a first estimate of the flying qualities of a hypersonic speceplane along its ascent and re-entry trajectory. In conclusion, the vehicle is unstable and, consequently, it needs to be controlled if we desire to perform a stable flight and achieve the mission objectives. An advanced control system will be designed in the next chapter. 


\section{6}

\section{Control-System Design}

A space vehicle needs to be equipped with a Guidance, Navigation and Control (GNC) system to ensure that the vehicle flies its nominal trajectory. The nominal trajectory of a vehicle is defined starting from the initial conditions and considering the trajectory constraints and end conditions. The nominal trajectory has to be defined in such a way that the vehicle is able to execute the specified manoeuvres and achieve the mission objectives even in presence of unforeseen disturbances. This is the main task of a GNC system.

Figure 6.1 gives a schematic representation of the GNC system. The guidance system has the objective of generating the steering commands taking into account constraints, ending conditions and reference state. The latter is provided by the navigation system by means of sensors and theoretical models. The objective of the control system is to control the motion about the vehicle's CoM so that the actual attitude and angular rates equal the commanded ones. In this way, the steering commands, given by the guidance system, are performed by control devices, such as control surfaces, TVC and RCS thrusters. They influence the trajectory of the vehicle by modifying the magnitude or the direction of the external forces, which are known as control forces. If the latter do not pass through the CoM, they generate control moments about the CoM that serve to control the attitude of the vehicle. The EoM provide the relation between the trajectory of the vehicle and the external forces and moments.

From the stability and flying-qualities analyses performed in the preceding chapter, it was demonstrated that the FSS-1 spaceplane is not dynamically stable. Thus, it needs to be equipped with a control system that stabilises it. In this way, the safety of flight can be ensured and the objectives of the mission can be reached. In this thesis work, the control system has an essential role and needs to be studied, while the guidance and navigation systems can be assumed to be ideal systems: the guidance system provides the exact commands required to fly the nominal trajectory; and the navigation system transfers all the data to the guidance and control systems with negligible errors. In the past sixty years several control systems have been developed. The classical control concepts, such as feedback control and optimal control theory, have been widely adopted. A well-known optimal control technique is the Linear Quadratic Regualtor (LQR).

However, the classical control methodologies can lack robustness, stability and/or require high control effort, so advanced control system were developed to solve these limitations. To this end several advanced controller have been designed, such as non-linear dynamic inversion [Miller, 2011], model predictive control [Camacho and Alba, 2007], adaptive control [Kaufman et al., 1998], sliding mode control [Young et al., 1999], and reinforcement learning [Lewis et al., 2012]. One in particular results to be the most suitable for this thesis work: the adaptive control system. It is convenient for our analyses becuase it was already proven to work for HORUS during the re-entry flight, from which the FSS-1 is derived. Also, the mathematics behind this algorithm is quite elegant and easy to implement. It is characterised by low sensitivity to any kind of disturbances thanks to its adaptive gains, resulting in enhanced robustness and performance of the system. Moreover, noteworthy supporting material is available, such as Mooij [1998b], [1998a], [2017] and [2018]. However, a control system is generally designed starting from a simple method and, afterwards, improving it with a more advanced system. Thus, the LQR controller is chosen as benchmark controller, being one of the most commonly used since it is quite simple to understand, formulate and implement.

Section 6.1 introduces the key concepts of the feedback control. Also, the performance indices that will serve for the analysis of the control-system design are introduced in Section 6.1.1. Section 6.2 details the optimal control theory and the well-known optimal control technique, which is the LQR controller. It is followed by 


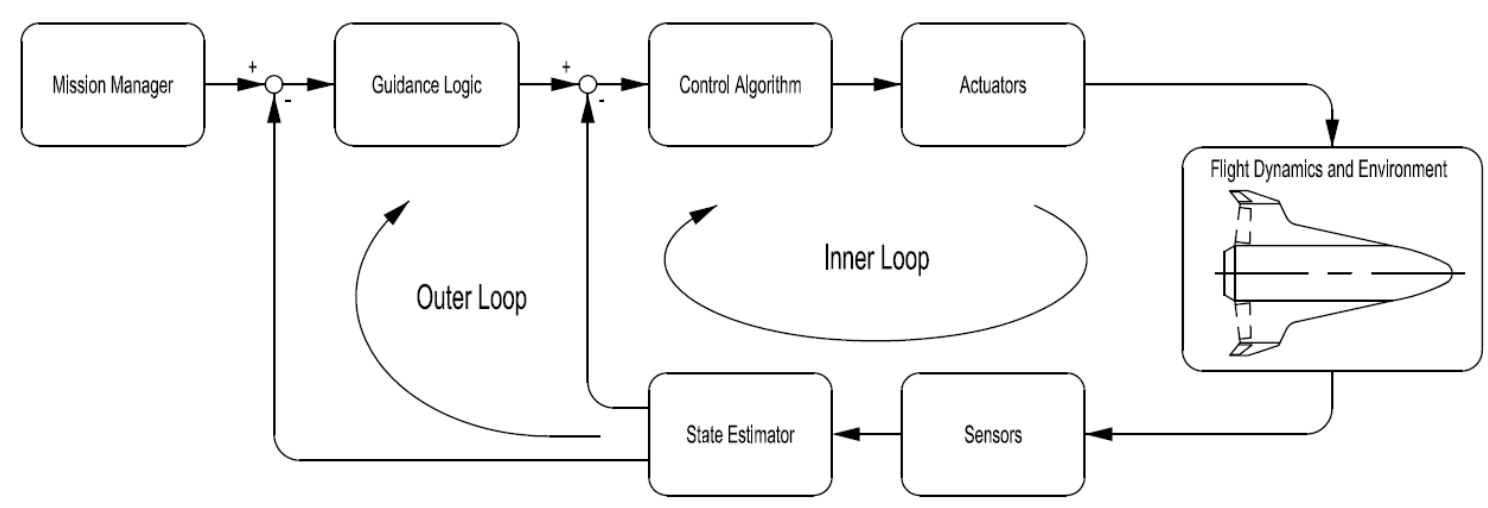

Figure 6.1: Schematic representation of a GNC system. [Mooij, 2015]

the definition of the LQR design for our reference mission. Section 6.3 exposes the theory of the adaptive control laws and describes the design of this advanced controller. Finally, once the control system has computed the commands, the commanded moments need to be allocated to the active actuators. To this end, the control allocator algorithm is used, which is presented in Section 6.4.

\subsection{Feedback Control}

Feedback control is a common and powerful tool in the design of a control system to make the controller less sensitive to external disturbances. A control system with feedback loop is generally known as closed-loop control. As shown in Figure 6.1, the controller receives as input the difference between commanded signal and the measured state of the system, which is called plant. This difference gives the error that the controller needs to correct by providing a new input to the system. In this section the main principles are exposed on the basis of the theory presented by Ogata [2010].

The general working principle of the feedback control is based on the linearised equations of motion written in state-space form as presented in Section 4.7, which yields to:

$$
\dot{\mathbf{x}}=\mathbf{A x}+\mathbf{B u}
$$

which is called dynamics equation. Here, $\mathbf{x}$ is the $\mathrm{n} \times 1$ state deviation vector, $\mathbf{u}$ is the $\mathrm{m} \times 1$ control deviation vector, and $\mathbf{A}$ and $\mathbf{B}$ are the $\mathrm{n} \times \mathrm{n}$ state and $\mathrm{n} \times \mathrm{m}$ control coefficient matrices, respectively. Similarly, the output equation can be written as:

$$
\dot{\mathbf{y}}=\mathbf{C x}+\mathbf{D u}
$$

where $\mathbf{y}$ is the $\mathrm{k} \times 1$ output vector, $\mathbf{C}$ and $\mathbf{D}$ are the $\mathrm{k} \times \mathrm{n}$ output and $\mathrm{k} \times \mathrm{m}$ direct transmission matrices, respectively. The coefficients of the matrices $\mathbf{A}, \mathbf{B}, \mathbf{C}$, and $\mathbf{D}$, which can be calculated by means of the linearised equations of motion, are constant. For this reason the system is said to be Linear-Time-Invariant (LTI). In this report, it is assumed that the output vector is identical to the state vector or a combination of the state variables of the system, so $\mathbf{D}$ is a null matrix.

As shown in Chapter 5, the open-loop behaviour of a system can be examined by calculating the eigenvalues and eigenvectors of the state matrix A. Thus, the functioning principle of the feedback control is based on the consideration that the behaviour of the system can be modified by changing this eigenvalues and eigenvectors.

The feedback loop can consist in either output feedback or state feedback. Since the linear system that describes the motion of the reference vehicle is based on the state vector, only state feedback is treated. For the state feedback, the control law is:

$$
\mathbf{u}=-\mathbf{K x}
$$

with $\mathbf{K}$ being the gain matrix. Substituting the control law in Eq. 6.1, it yields:

$$
\dot{\mathbf{x}}=(\mathbf{A}-\mathbf{B K}) \mathbf{x}
$$

Thus, the eigenvalues and the corresponding eigenmotion of the closed-loop system can be calculated by solving the following characteristic equation:

$$
\operatorname{det}|\mathbf{A}-\mathbf{B K}-\lambda \mathbf{I}|=0
$$




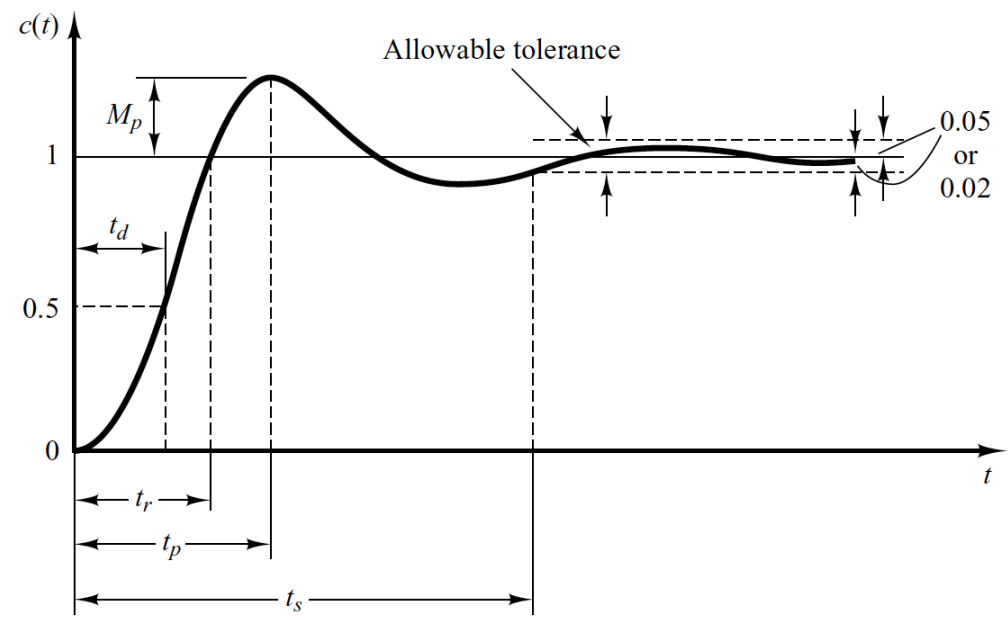

Figure 6.2: Analysis of the transient response to a unit-step input [Ogata, 2010].

where it is possible to notice that, varying the gain matrix $\mathbf{K}$, the eigenvalues of the closed-loop system can be changed.

The parameters that define the performance of these control systems are the gains that can be determined by pole placement or other mathematical optimization criteria. For instance, the linear quadratic cost criterion is used in the well-known Linear Quadratic Regualtor $(L Q R)$ control method, which will be described in Section 6.2 .

\subsubsection{Performance Indices}

Generally, the performance of a control system is characterized in terms of the transient response to a unitstep input. The unit-step input is quite easy to generate and any arbitrary input can be decomposed into a series of sequential step inputs. Thus, if the response to a unit-step input is known, the response to any kind of input can be obtained. Figure 6.2 illustrates the performance characteristics of a control system, which are:

- Delay time, $t_{d}$ : time required for the response to reach half of the final value for the first time;

- Rise time, $t_{r}$ : time required for the response to rise from $0 \%$ to $100 \%$ of its final value, for under-damped second-order systems, and from the $10 \%$ to $90 \%$ of its final value, for over-damped systems;

- Peak time, $t_{p}$ : time required for the response to reach the first peak of the overshoot;

- Maximum overshoot, $M_{p}$ : maximum peak value reached by the response measured from unity or given as maximum percent overshoot if the final steady-state value of the response differs from unity;

- Settling time, $t_{s}$ : time required for the response to reach and stay within a range (usually $2 \%$ or $5 \%$ ) about the final value.

Generally, it is desired that the transient response is fast and well-damped, i.e., maximum overshoot and rise time simultaneously small. To obtain it, the damping ratio should be $0.4 \leq \zeta \leq 0.8$ for a second-order system. Differently, $\zeta<0.4$ indicates a transient response with excessive overshoot and $\zeta>0.8$ indicates a slow and weak response. These performance indices are also used to specify some of the MIL requirements for controllability, e.g., the roll control effectiveness, $t_{30}$, is defined as the maximum allowed time necessary to the system to generate a bank-angle command (see Table 5.4).

However, the performance of the controller can be also formulated by means of objective functions. The goals of a control system are:

- to minimise the state deviation between the plant and the control commands

- to minimise the control effort necessary to affect the plant's behaviour.

It follows that the integrated control error and the integrated control effort can give information about the performance of the control action and about how optimal it is.

For the longitudinal control system design, the two objectives are expressed as the integrated angle-of- 

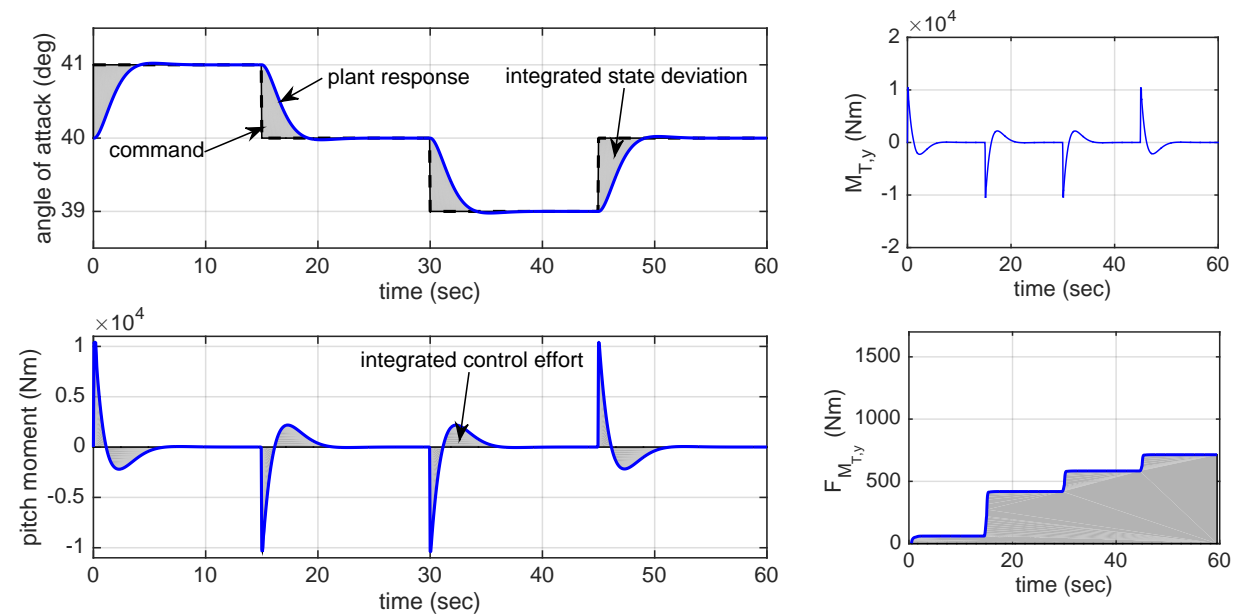

Figure 6.3: Graphical representation of the controller performance indices from a mathematical point of view [Mooij, 2017].

attack deviation and integrated pitch-moment fraction, thus:

$$
\sum_{\alpha_{e r r}}=\int_{0}^{t}\left|\alpha_{c}(t)-\alpha_{p}(t)\right| \mathrm{d} t \quad \sum_{\eta_{y}}=\int_{0}^{t}\left|\eta_{y}(t)\right| \mathrm{d} t
$$

where the integrated control effort can also be linked to the actual usage of the actuators, so the integrated elevator deflection, integrated thrust elevation, and integrated pitch thruster moment, i.e., $\sum_{\delta_{e}}=\int_{0}^{t}\left|\delta_{e}(t)\right| \mathrm{d} t$, $\sum_{\epsilon_{T}}=\int_{0}^{t}\left|\epsilon_{T}(t)\right| \mathrm{d} t, \sum_{M_{T, y}}=\int_{0}^{t}\left|M_{T, y}(t)\right| \mathrm{d} t$ can be added.

For the lateral control system design, the objective functions can expressed as the integrated bank-angle deviation and integrated roll and yaw moment fractions, thus:

$$
\sum_{\sigma_{\text {err }}}=\int_{0}^{t}\left|\sigma_{c}(t)-\sigma_{p}(t)\right| \mathrm{d} t \quad \sum_{\eta_{x}}=\int_{0}^{t}\left|\eta_{x}(t)\right| \mathrm{d} t \quad \sum_{\eta_{z}}=\int_{0}^{t}\left|\eta_{z}(t)\right| \mathrm{d} t
$$

where the roll and yaw moment fractions can be linked to the activity of the actuators by defining the integrated aileron and rudder deflections, roll and yaw thruster activity, and thrust azimuth, $\sum_{\delta_{a}}=\int_{0}^{t}\left|\delta_{a}(t)\right| \mathrm{d} t$, $\sum_{\delta_{r}}=\int_{0}^{t}\left|\delta_{r}(t)\right| \mathrm{d} t, \sum_{M_{T, x}}=\int_{0}^{t}\left|M_{T, x}(t)\right| \mathrm{d} t, \sum_{M_{T, z}}=\int_{0}^{t}\left|M_{T, z}(t)\right| \mathrm{d} t, \sum_{\psi_{T}}=\int_{0}^{t}\left|\psi_{T}(t)\right| \mathrm{d} t$. However, since the control system directly commands the moment fractions while the actuator activity also depends also on the actuator assignment, when we desire to study the performance of the control system it is better to express the integrated control effort as the integrated moment fraction.

Also, it is desirable that the actuator oscillations are as small and rare as possible. The cumulative moving standard deviation is adopted to measure the oscillations or the discrete changes in the controls. Consider a subset $j$ of $n_{s}$ samples out of $N$ samples of an arbitrary signal $u$, the moving mean is defined as $\bar{y}_{j}=$ $\frac{1}{n_{s}} \sum_{i=j}^{j+n_{s}-1} u_{i}$, where $j$ runs from $1+n_{s} / 2$ to $N-n_{s} / 2$. The squared deviation from this mean at the midpoint of the subset $j$ is equal to $s_{u, j}=\left(u_{j+n_{s} / 2}-\bar{y}_{j}\right)^{2}$. Then, the cumulative standard deviation is defined as:

$$
F_{u_{j}}=\sqrt{\frac{1}{N-n_{s}-1} \sum_{k=1}^{j} s_{k}}
$$

whose gradient measures the local oscillations of $u$, i.e., $\sum_{F_{u_{j}}}=\int_{0}^{t}\left|F_{u_{j}}(t)\right| \mathrm{d} t$. The signal $u$ can be the activity of any of control variables. This measure should be as small as possible for smoother control. It should be noticed that this index is only indicative, because it depends on the dimension of the sample interval chosen and the mean-control-value progression. However, it is functional for the optimisation of the controller.

Figure 6.3 provides a graphical representation of the above indices, which equal the gray areas, for a sequence of angle-of-attack step commands during the HORUS re-entry flight when only reaction control is available. Thus, it is possible to understand that the smaller these areas, i.e., the smaller the performance indices, the better the controller performance. On the right of this figure, a graphical representation of the integrated actuator oscillations is provided, where it is shown that the jumps in control moment lead to a rapid increase of $\sum_{F_{M_{T, y}}}$. 


\subsection{Optimal Control Theory}

An extensively-used method to determine the feedback matrix $\mathbf{K}$ is to use the optimal control theory, where $\mathbf{K}$ is calculated by solving an optimisation problem. The optimisation problem has the following formulation. Determine the control function $\mathbf{u}(t)$ that minimises the cost function $J$, while the state variables satisfy the dynamic constraints along with the associated boundary conditions:

$$
\begin{gathered}
\min _{\mathbf{u}(t) \in U} J=\int_{t_{0}}^{t_{f}} \mathscr{L}[\mathbf{x}(t), \mathbf{u}(t), t] \mathrm{d} t \\
\text { subject to: } \dot{\mathbf{x}}=\mathbf{f}[\mathbf{x}, \mathbf{u}, t]
\end{gathered}
$$

where $\mathscr{L}$ is the Lagrangian of the problem. A well-known optimal control technique that can solve this optimization problem is the Linear Quadratic Regulator (LQR), whose basic principles are presented in the next section on the basis of the information offered by Gopal [1989].

\subsubsection{Linear Quadratic Regulator (LQR)}

In the LQR controller, the indirect method Quadratic Optimal Control is used to define the feedback matrix $\mathbf{K}$ by minimizing the following quadratic cost function $J$ :

$$
\begin{gathered}
\mathbf{J}=\int_{0}^{\infty}\left(\mathbf{x}^{T} \mathbf{Q} \mathbf{x}+\mathbf{u}^{T} \mathbf{R u}\right) \mathrm{d} t \\
\text { subject to: } \dot{\mathbf{x}}=\mathbf{A x}+\mathbf{B u} \text { and } \dot{\mathbf{u}}=-\mathbf{K} \mathbf{x}
\end{gathered}
$$

with $\mathbf{x}^{T} \mathbf{Q x}$ being the control deviation and $\mathbf{u}^{T} \mathbf{R u}$ the control effort. $\mathbf{Q}$ is a real positive semi-definite matrix and $\mathbf{R}$ is a real symmetric positive definite matrix, so that any value of $\mathbf{x}$ and $\mathbf{u}$ will not contribute negatively to $\mathbf{J}$. To determine $\mathbf{Q}$ and $\mathbf{R}$, Bryson's Rule provides a good first choice by defining:

$$
\begin{aligned}
& \mathbf{Q}=\operatorname{diag}\left\{\begin{array}{llll}
\frac{1}{\Delta x_{1, \max }^{2}} & \frac{1}{\Delta x_{2, \text { max }}^{2}} & \cdots & \left.\frac{1}{\Delta x_{n, \text { max }}^{2}}\right\}
\end{array}\right. \\
& \mathbf{R}=\operatorname{diag}\left\{\begin{array}{llll}
\frac{1}{\Delta u_{1, \max }^{2}} & \frac{1}{\Delta u_{2, \max }^{2}} & \cdots & \frac{1}{\Delta u_{m, \max }^{2}}
\end{array}\right\}
\end{aligned}
$$

where $\Delta x_{i, \text { max }}$ and $\Delta u_{j, \text { max }}$ are the maximum standard deviation of the $i$-th state variable and of the $j$-th control variable, respectively. The weight of control deviation and control effort can be modified by changing the matrices $\mathbf{Q}$ and $\mathbf{R}$. Giving more weight to the control deviation results in a faster response, while more weight to the control effort results in smaller control signals.

The feedback matrix $\mathbf{K}$ can be determined using the stability analysis according to Lyapunov. It results:

$$
\mathbf{K}=\mathbf{R}^{-1} \mathbf{B}^{T} \mathbf{P}
$$

with $\mathbf{P}$ being a positive definite matrix that can be computed from the matrix Riccati equation:

$$
\mathbf{A}^{T} \mathbf{P}+\mathbf{P A}-\mathbf{P B R} \mathbf{R}^{-1} \mathbf{B P}+\mathbf{Q}=\mathbf{0}
$$

This equation can be solved with standard algorithm, available between control-system design tools in Matlabß.

The LQR is generally advisable for MIMO (Multiple Inputs and Multiple Outputs) systems. The advantages of using LQR are the ease of the implementation of the control law in flight-simulation software and the low computational loads that are required for on-line simulation. However, when the states of the system are frequently changing with time or disturbances act on the system, the LQR is not generally capable to properly control the vehicle during the whole flight and a more advanced controller is required. Nonetheless, LQR has been used yet for spaceplanes control in ascent and/or re-entry flight to have a first estimate of the controllability of the system. Thus, it can be generally used as a benchmark controller, as done in the work by Brinkman [2017].

\subsubsection{LQR for Reference Vehicle}

The attitude control system is designed around the reference trajectory by using the linearised EoM. This set of equations allows to decouple the symmetric and asymmetric eigenmotion. This decoupling is used as 


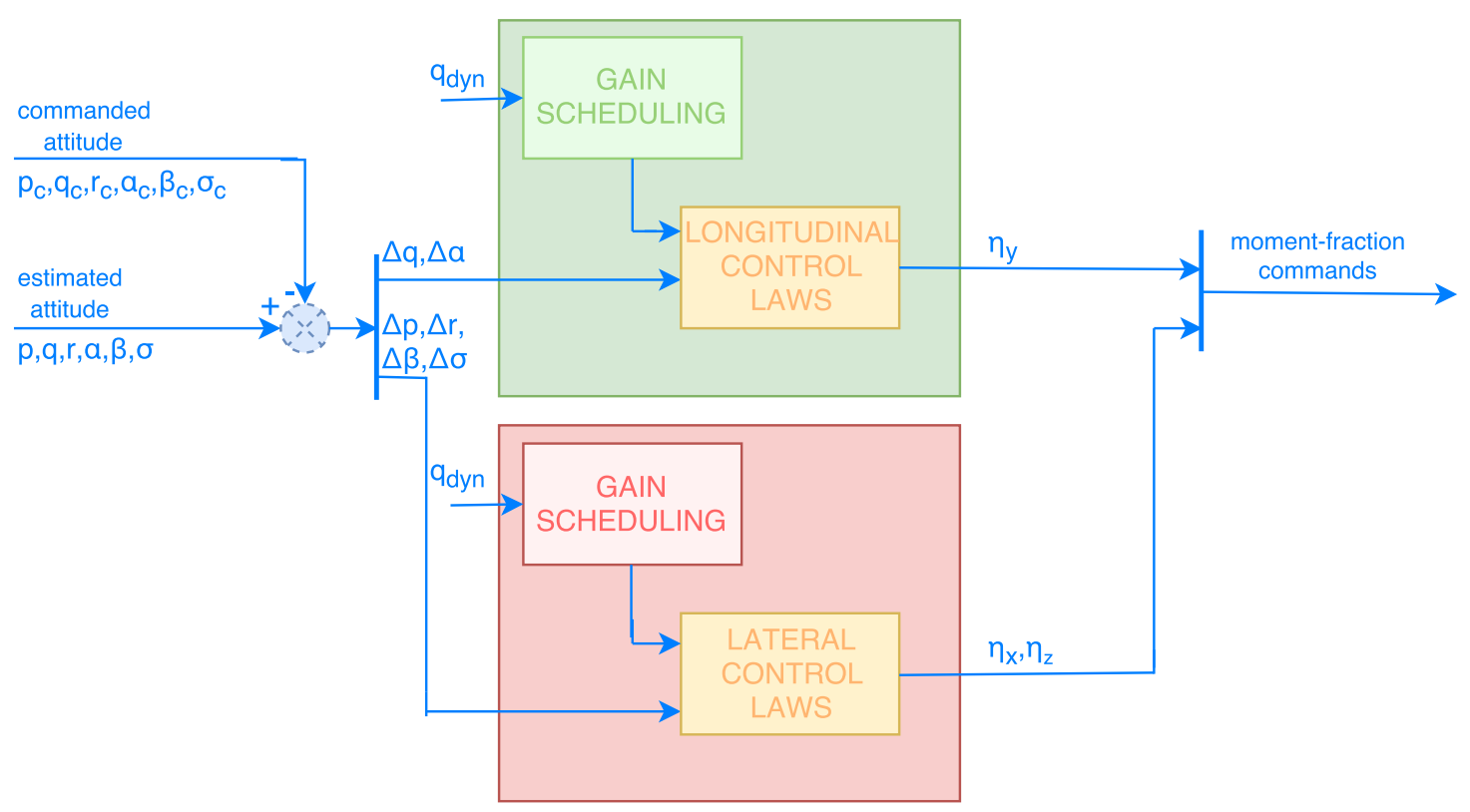

Figure 6.4: Schematic layout of the LQR controller (to be linked to the software architecture shown in Figure B.1).

advantage for the control system design. Indeed, the longitudinal and lateral motion can be decoupled by separating the controller in two main parts, which are the longitudinal controller and the lateral controller. These two parts are implemented independently and then integrated together to form a complete controller. In Figure 6.4, the layout of the benchmark controller has been schematically depicted, which is to be linked to the "grand scheme" of the software architecture shown in Figure B.1.

The controller is composed by an inner loop and an outer loop that serve for longitudinal and lateral control, respectively. The former is constituted of longitudinal trim and control. The trim is executed by means of the body-flap deflection, with the support of the TVC in ascent, and the elevons in descent. The longitudinal control is realised by elevator and TVC, in ascent, or pitch thrusters, in re-entry flight. The lateral control represents the outer loop, given the high frequency of the rotational dynamics, for which only small moments are required to stabilize the vehicle. The lateral control is realised by aileron, rudder and TVC in ascent, or roll and yaw thrusters in re-entry flight. The command input is the angle of attack for longitudinal commands and bank angle for lateral-directional commands.

In the following the control design is detailed for our reference vehicle and mission. As mentioned before, the used control variables are the moment fractions. So, the controller commands directly the required moments about the three axes regardless the active actuators. The need for a controller using moment-fractions as control variables can be understood if we consider that, first, the control actuators are different for ascent and re-entry and, secondly, they are activated depending on their efficiency as function of the dynamic pressure. During the re-entry the control modes are set on the basis of the control scheme used for the Space Shuttle, which is shown in Figure 6.5. Similarly, along the ascent trajectory for $\bar{q}<5000 \mathrm{~Pa}(M>5)$ the control surfaces becomes inefficient and the TVC must be used to control the vehicle. Thus, the moment-fraction allow to design a controller that can be used globally along the reference trajectory, independently of the active actuators. Afterwards, the commanded moments are allocated to the active actuators.

For the control-system verification, the control performance is compared with the reference data about the attitude control of HORUS obtained by Rijnsdorp [2017]. In this case, the same model used by Rijnsdorp [2017] is adopted so that the acceptance test can be performed. Once it is verified, it is used as benchmark to verify the results for FSS-1 control in the ascent and re-entry trajectory and, afterwards, to verify the adaptive control system, as presented in Section B.2.2.

\section{Moment-fraction LQR}

To design a control system that can function along the whole trajectory, it possible to directly command the required moments about each axis, instead of the six actuators, making the controller less dependent on the operational conditions. The commanded moments are defined by the moment fractions and the maximum available moments. 


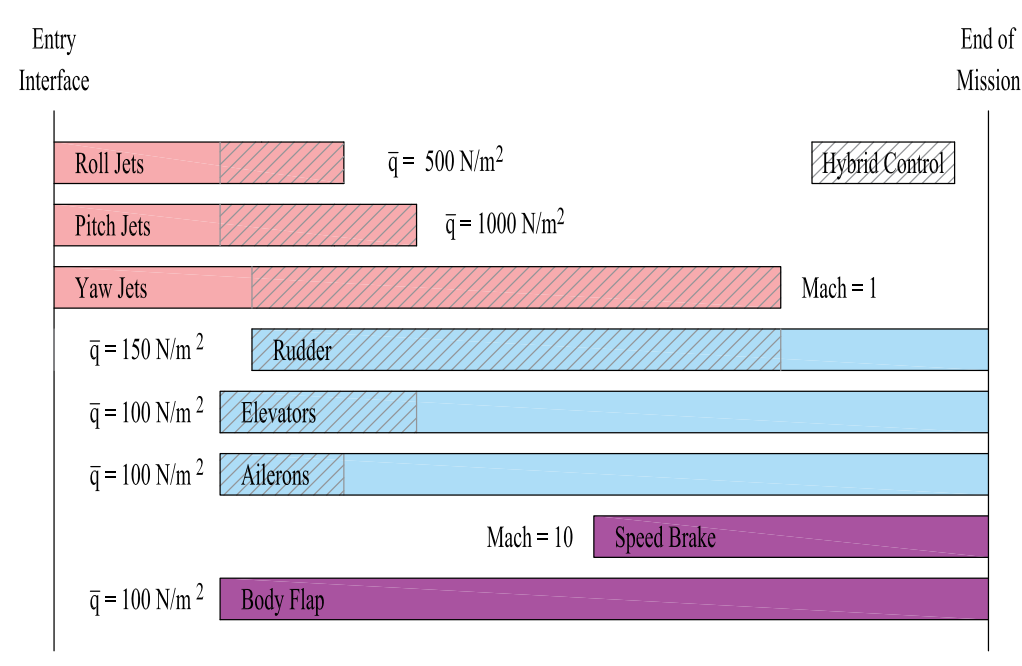

Figure 6.5: Control modes for FSS-1 (and HORUS) during the re-entry flight. [Mooij, 2018]

The equations for $\Delta \dot{p}, \Delta \dot{q}, \Delta \dot{r}$ of the linearised EoM set are defined as:

$$
\begin{aligned}
& \Delta \dot{p}=\frac{\bar{q}_{0} S_{\text {ref }} b_{\text {ref }}}{I_{x x}} \frac{\partial C_{l}}{\partial \beta} \Delta \beta+\frac{\mathscr{L}_{\text {max }}}{I_{x x}} \eta_{x} \\
& \Delta \dot{q}=\frac{\bar{q}_{0} S_{r e f} c_{r e f}}{I_{y y}} \frac{\partial C_{m}}{\partial \alpha} \Delta \alpha+\frac{\mathscr{M}_{\text {max }}}{I_{y} y} \eta_{y} \\
& \Delta \dot{r}=\frac{\bar{q}_{0} S_{\text {ref }} b_{\text {ref }}}{I_{z z}} \frac{\partial C_{n}}{\partial \beta} \Delta \beta+\frac{\mathscr{N}_{\text {max }}}{I_{z z}} \eta_{z}
\end{aligned}
$$

where $\mathscr{L}_{\text {max }}, \mathscr{M}_{\text {max }}$, and $\mathscr{N}_{\text {max }}$ represent the maximum available moments.

To compute the maximum available moments, one needs to realise that the positive and negative maximum available moments about the roll and pitch axes are not the same and it is not known in advance which one is larger in modulus. Thus, it is chosen to pick the minimum of the two maximum coefficients, so that the saturation is avoided in case the elevons are also used for trim. The maximum available moments along the yaw axis is simpler to determine since only one rudder at a time is used. In this way, one can calculate the maximum available moment coefficients $C_{l, \max }, C_{m, \max }$, and $C_{n, \max }$, from which the maximum available aerodynamic moments can be derived. So, on the basis of Figure 6.5, the maximum moment capability along

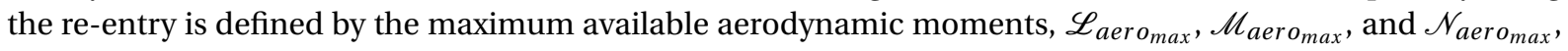
and the maximum available propulsion moments, $M_{T, x_{\max }}, M_{T, y_{\max }}, M_{T, z_{\max }}$, as follows:

$$
\begin{aligned}
& \mathscr{L}_{\text {max }}= \begin{cases}M_{T, x_{\text {max }}} & \text { if } 0 \leq \bar{q}<100 \mathrm{~N} / \mathrm{s}^{2} \\
\mathscr{L}_{\text {aero } \max }+M_{T, x_{\max }} & \text { if } 100 \leq \bar{q}<500 \mathrm{~N} / \mathrm{s}^{2} \\
\mathscr{L}_{\text {aero } o_{\text {max }}} & \text { if } \bar{q} \geq 500 \mathrm{~N} / \mathrm{s}^{2}\end{cases} \\
& \mathscr{M}_{\text {max }}= \begin{cases}M_{T, y_{\max }} & \text { if } 0 \leq \bar{q}<100 \mathrm{~N} / \mathrm{s}^{2} \\
\mathcal{M}_{\text {aero }} \text { max } \\
\mathscr{M}_{\text {aero } o_{\max }}+M_{T, y_{\max }} & \text { if } 100 \leq \bar{q}<1000 \mathrm{~N} / \mathrm{s}^{2} \\
\text { if } \bar{q} \geq 1000 \mathrm{~N} / \mathrm{s}^{2}\end{cases}
\end{aligned}
$$

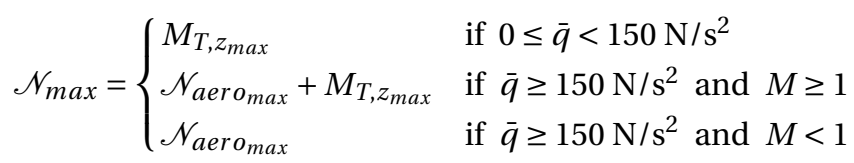


Similarly, the maximum moment capability along the ascent trajectory can be defined:

$$
\begin{aligned}
& \mathscr{L}_{\text {max }}= \begin{cases}\mathscr{L}_{\text {aero }}+M_{T, x_{\text {max }}} & \text { if } \bar{q} \geq 5000 \mathrm{~N} / \mathrm{s}^{2} \\
M_{T, x_{\max }} & \text { if } 0 \leq \bar{q}<5000 \mathrm{~N} / \mathrm{s}^{2}\end{cases} \\
& \mathscr{M}_{\text {max }}= \begin{cases}\mathscr{M}_{\text {aero }}+M_{\text {max }} \\
M_{T, y_{\max }} & \text { if } \bar{q} \geq 5000 \mathrm{~N} / \mathrm{s}^{2}\end{cases} \\
& \mathscr{N}_{\text {max }}= \begin{cases}\mathscr{N}_{\text {aero }} \text { if } 0 \leq \bar{q}<5000 \mathrm{~N} / \mathrm{s}^{2} \\
M_{T, z_{\max }}+M_{T, z_{\text {max }}} & \text { if } \bar{q} \geq 5000 \mathrm{~N} / \mathrm{s}^{2}\end{cases}
\end{aligned}
$$

Once the controller compute the fraction of maximum available moments required to correct the control errors, the commanded moments can be computed as following:

$$
\mathscr{L}_{c m d}=\eta_{x} \mathscr{L}_{\text {max }}, \quad \mathscr{M}_{c m d}=\eta_{y} \mathscr{M}_{\text {max }}, \quad \mathscr{N}_{c m d}=\eta_{z} \mathscr{N}_{\text {max }}
$$

where $\eta_{x}, \eta_{y}$, and $\eta_{z}$ are the moment fractions about the $x$-, $y$-, and $z$-axis. The commanded moments will be afterwards collocated to the active actuators throughout a procedure that is known as actuator assignment, detailed in Chapter 6.4.

From the model described by Eqs. from (6.17) to (6.17), the longitudinal and lateral state-feedback control laws can be defined. The longitudinal control law is:

$$
\Delta u=\eta_{y}=-K_{y q} \Delta q-K_{y \alpha} \Delta \alpha
$$

and the lateral control law can be written as:

$$
\Delta \mathbf{u}=\left(\begin{array}{l}
\eta_{x} \\
\eta_{z}
\end{array}\right)=-\left[\begin{array}{llll}
K_{x p} & K_{x r} & K_{x \beta} & K_{x \sigma} \\
K_{z p} & K_{z r} & K_{z \beta} & K_{z \sigma}
\end{array}\right]\left(\begin{array}{c}
\Delta p \\
\Delta r \\
\Delta \beta \\
\Delta \sigma
\end{array}\right)=-\mathbf{K} \Delta x
$$

The weighting matrices, calculated by following the Bryson's rule, are for the longitudinal control:

$$
\mathbf{Q}=\operatorname{diag}\left\{\frac{1}{\Delta q_{\text {max }}^{2}} \quad \frac{1}{\Delta \alpha_{\text {max }}^{2}}\right\}, \quad \mathbf{R}=\frac{1}{\eta_{y, \text { max }}^{2}}
$$

and for lateral control:

$$
\mathbf{Q}=\operatorname{diag}\left\{\begin{array}{llll}
\frac{1}{\Delta p_{\max }^{2}} & \frac{1}{\Delta r_{\max }^{2}} & \frac{1}{\Delta \alpha_{\max }^{2}} & \frac{1}{\Delta \sigma_{\max }^{2}}
\end{array}\right\}, \quad \mathbf{R}=\operatorname{diag}\left\{\frac{1}{\eta_{x, \max }^{2}} \quad \frac{1}{\eta_{z, \max }^{2}}\right\}
$$

where the maximum allowed amplitudes of the state variables are $\Delta \alpha_{\max }=1^{\circ}, \Delta \beta_{\max }=\Delta \sigma_{\max }=2^{\circ}$, and $\Delta p_{\text {max }}=\Delta q_{\text {max }}=\Delta r_{\text {max }}=10^{\circ} / \mathrm{s}$, and of the control variables are $\Delta \eta_{x, \text { max }}=\Delta \eta_{y, \max }=\Delta \eta_{z, \text { max }}=1$. They are chosen so that the controller is able to stabilise the vehicle for a step response in angle of attack, for the longitudinal controller, and in sideslip angle and/or bank angle, for the lateral controller. These values are identified from response tests performed during an iterative process to tune the control system. For instance, it was noted that smaller maximum allowable state deviations can induce a better plant response, but at the expense of higher deflection angles and/or thruster moments, which can lead to saturation of the actuators.

During the response tests it was noticed that the allowable rotational-rate errors need to decreased when input are commanded along the ascent trajectory, because the actual values leads to slower response. Indeed, if smaller allowable rotational-rate errors are set up, the control is tighter and, consequently, the response faster. So, the maximum allowable state error are now determined: $\Delta \alpha_{\max }=1^{\circ}, \Delta \beta_{\max }=\Delta \sigma_{\max }=2^{\circ}$, and $\Delta p_{\max }=\Delta q_{\max }=\Delta r_{\max }=4^{\circ} / \mathrm{s}$. Furthermore, while testing the performance of the control system, it was confirmed that along the ascent trajectory the control surfaces are more and more ineffective with decreasing dynamic pressure. In particular, for $\bar{q}<5000$, they can no longer significantly influence the state variables.

The gains are computed off-line for the design points along the reference trajectory and they are stored on-board as function of the dynamic pressure. If the whole reference trajectory was available, it would be possible to compute the gains for a number of points of the trajectory so that the actual gains of any point of the trajectory can be computed by linear interpolation for the local value of dynamic pressure. This process is called gain scheduling. 


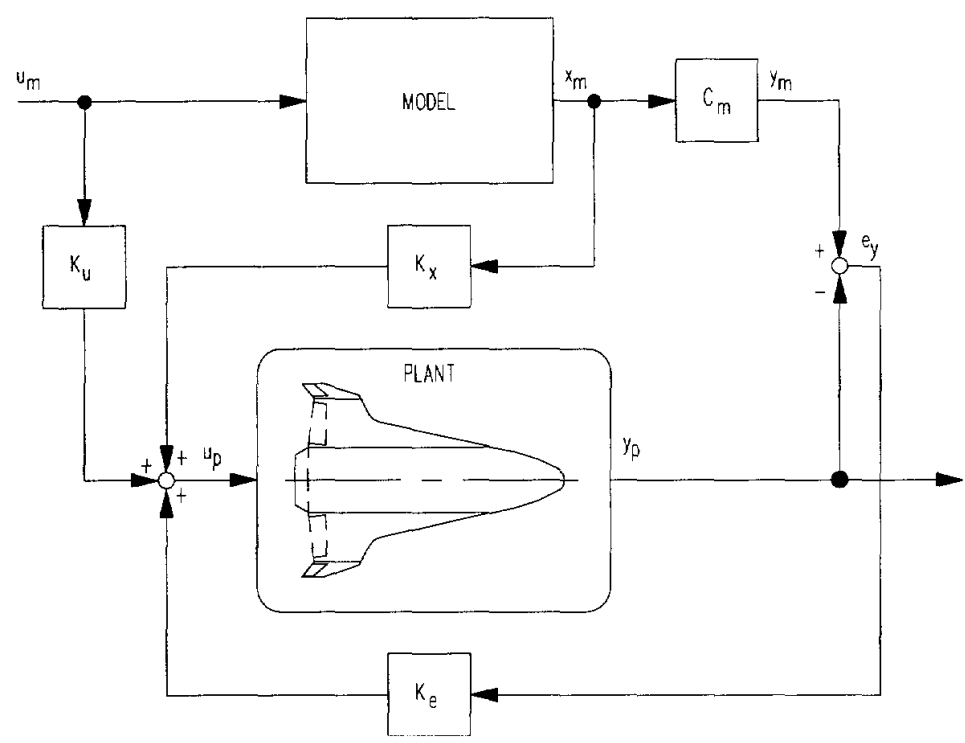

Figure 6.6: Simple adaptive control system: simplified model [Mooij, 1998a].

\subsection{Model Reference Adaptive Control}

In section 6.3.1, an overview of the mathematical model of the simple adaptive controller (SAC) is offered on the basis of the information provided by Kaufman et al. [1998], to which the interested reader is referred for further details and derivations. Section 6.3.2 describes the reference model. Finally, the baseline adaptivecontrol design is studied and presented in Section 6.3.3.

\subsubsection{Theory}

The motion of a spaceplane can be modelled with a linearised set of EoM. However, this model cannot perfectly described the real system, thus some non-linearities should be accounted for. As discussed in Chapter 4 , the linear model of the plant can be defined by the state-space model. The set of linearised EoM in statespace form is:

$$
\dot{\mathbf{x}}_{\mathbf{m}}=\mathbf{A}_{\mathbf{m}} \mathbf{x}_{\mathbf{m}}+\mathbf{B}_{\mathbf{m}} \mathbf{u}_{\mathbf{m}}
$$

where $\mathbf{x}_{\mathbf{m}}$ is the $n \times 1$ model state vector, $\mathbf{u}_{\mathbf{m}}$ is the $m \times 1$ model control vector, and $\mathbf{A}_{\mathbf{m}}$ and $\mathbf{B}_{\mathbf{m}}$ the $n \times n$ model state matrix and $n \times m$ model control matrix, respectively. Similarly, the output equation is:

$$
\mathbf{y}_{\mathbf{m}}=\mathbf{C}_{\mathbf{m}} \mathbf{x}_{\mathbf{m}}
$$

where $\mathbf{y}_{\mathbf{m}}$ is the $q \times 1$ model output vector and $\mathbf{C}_{\mathbf{m}}$ is the $q \times n$ model output matrix. As can be noticed, the subscript $m$ is added to the variables, underlining the fact that this set of equations describes the model of the plant. Note that $\mathbf{x}, \mathbf{u}$, and $\mathbf{y}$ are function of time, thus they should be written as $\mathbf{x}(t), \mathbf{u}(t), \mathbf{y}(t)$. However, the $t$ is omitted for the sake of convenience.

Differently, the original spaceplane, commonly called plant, is described by the following set of non-linear differential equation:

$$
\begin{aligned}
\dot{\mathbf{x}}_{\mathbf{p}} & =\mathbf{f}\left(t, \mathbf{x}_{\mathbf{p}}, \mathbf{u}_{\mathbf{p}}\right) \\
\mathbf{y}_{\mathbf{p}} & =\mathbf{g}\left(\mathbf{x}_{\mathbf{p}}, \mathbf{u}_{\mathbf{p}}\right)
\end{aligned}
$$

where $\mathbf{x}_{\mathbf{p}}$ is the $n_{p} \times 1$ plant state vector, $\mathbf{u}_{\mathbf{p}}$ is the $m \times 1$ plant control vector, $\mathbf{y}_{\mathbf{p}}$ is the $q \times 1$ plant output vector, f and $\mathbf{g}$ are the $n_{p} \times 1$ and $q \times 1$ plant vector functions. The subscript $p$ is added to the variables to underline the fact that this set of equations describes the behaviour of the non-linear plant. It can be noticed from the dimensions of the vectors given above that the number of model inputs and outputs must be equal to the number of plant inputs and outputs.

Figure 6.6 shows a simplified scheme of the SAC. It is shown that the inputs to the plant are the model input $\mathbf{u}_{\mathbf{m}}$, the model state $\mathbf{x}_{\mathbf{m}}$, and the output error $\mathbf{e}_{\mathbf{y}}$, which is the difference between the model output and the plant output. The inputs to the plant are obtained by multiplying these variables $\mathbf{u}_{\mathbf{m}}, \mathbf{x}_{\mathbf{m}}$, and $\mathbf{e}_{\mathbf{y}}$ to the adaptive 
gains, which are, respectively, $\mathbf{K}_{\mathbf{u}}, \mathbf{K}_{\mathbf{x}}$, and $\mathbf{K}_{\mathbf{e}}$. These gains are adaptive in the sense that they vary with time, adapting their values to the changing conditions and leading to an improvement of the robustness and stability of the system. Thus, also $\mathbf{K}_{\mathbf{u}}(t), \mathbf{K}_{\mathbf{x}}(t)$, and $\mathbf{K}_{\mathbf{e}}(t)$ are function of time, but the $t$ is omitted in the formulation for the sake of convenience. We can conclude that, if the control system is properly designed, the inputs to the plant lead the outputs of the plant to equal the outputs of the model, that represent the reference desired trajectory.

The algorithm to compute the adaptive gains can be introduced. Defining a vector of the three measurable signals:

$$
\mathbf{r}=\left(\mathbf{e}_{\mathbf{y}}, \mathbf{x}_{\mathbf{m}}, \mathbf{u}_{\mathbf{m}}\right)^{T}
$$

and a matrix that combines the adaptive gains:

$$
\mathbf{K}_{\mathbf{r}}=\left[\mathbf{K}_{\mathbf{e}}, \mathbf{K}_{\mathbf{x}}, \mathbf{K}_{\mathbf{u}}\right]
$$

thus, the control law can be specified as:

$$
\mathbf{u}_{\mathbf{p}}=\mathbf{K}_{\mathbf{r}} \mathbf{r}
$$

The adaptive gains can be estimated by defining $\mathbf{K}_{\mathbf{r}}$ as the sum of an integral component and a proportional component, that are also time dependent:

$$
\mathbf{K}_{\mathbf{r}}=\mathbf{K}_{\mathbf{i}}+\mathbf{K}_{\mathbf{p}}
$$

with

$$
\begin{aligned}
\dot{\mathbf{K}}_{\mathbf{i}} & =\mathbf{e}_{\mathbf{y}} \mathbf{r}^{T} \mathbf{T}_{\mathbf{i}} \\
\mathbf{K}_{\mathbf{p}} & =\mathbf{e}_{\mathbf{y}} \mathbf{r}^{T} \mathbf{T}_{\mathbf{p}}
\end{aligned}
$$

where the weighting matrices $\mathbf{T}_{\mathbf{i}}$ and $\mathbf{T}_{\mathbf{p}}$ are a positive definite matrix and a positive semi-definite matrix, respectively.

The proportional-gain component of the adaptive gain vector $\mathbf{K}_{\mathbf{r}}$ influences directly the transient tracking behaviour, while the integral-gain component guarantees the asymptotic tracking. Also, Mooij [2017] offers a way to improve the damping of the system, including the error derivative to the output error vector, as follows:

$$
\mathbf{e}_{\mathbf{y}}=\mathbf{K}_{\mathbf{y}}^{T}\left(\mathbf{y}_{\mathbf{m}}-\mathbf{y}_{\mathbf{p}}\right)+\mathbf{K}_{\mathbf{y}}{ }^{T}\left(\dot{\mathbf{y}}_{\mathbf{m}}-\dot{\mathbf{y}}_{\mathbf{p}}\right)
$$

where $\mathbf{K}_{\mathbf{y}}^{T}$ and $\mathbf{K}_{\mathbf{y}}{ }^{T}$ are the proportional and the derivative output gains, respectively. However, calculating the numerical derivative of the outputs and tuning the related gains in multiple-output systems could be very tedious. Thus, an alternative expression for $\mathbf{e}_{\mathbf{y}}$ may be used:

$$
\mathbf{e}_{\mathbf{y}}=\mathbf{y}_{\mathbf{m}}-\mathbf{y}_{\mathbf{p}}=\mathbf{K}_{\mathbf{l q r}, \mathbf{c}}\left(\mathbf{C}_{\mathbf{m}} \mathbf{x}_{\mathbf{m}}-\mathbf{C}_{\mathbf{p}} \mathbf{x}_{\mathbf{p}}\right)
$$

where the output matrix is adjusted by pre-multiplying it with the optimal control-gain matrix, $\mathbf{K}_{\mathbf{l q r}, \mathbf{c}}$, obtained by solving the Algebraic Riccati Equation for the linearised EoM set of the reference model. In this way, a proper response is achieved by introducing a sufficient damping.

The adaptive control is possible when the adaptive system is state and gain bounded and presents an asymptotically stable output error. So, the plant is required to be almost strictly positive real (ASPR) or almost strictly passive (ASP). This can be realised if the following condition is respected: each control channel shall be single-input single-output (SISO). Determining whether a plant is ASPR or ASP is not so trivial and the full derivation can be found in Kaufman et al. [1998]. For this reason, an extension to the control algorithm to widen the class of systems is required. One possibility is to augment the plant with a feedforward compensator, while an other option is to apply the feedforward to both plant and reference model because the model feedforward helps to reach a zero steady-state error. These are appropriate solutions, although they result in a tedius laborious work. It was shown by Mooij [2014] that the non-linear system is ASP, when the controller directly commands the external moments, rather than the actuators themselves, i.e., when we design the controller to be SISO. In this way, the feedforward compensators are not required and the design effort is largely reduced. Examples of the spaceplanes and re-entry flight applications of the adaptive controller are presented by Mooij [1998a], [1998b], [2017], [2018], which constitute fundamental references for this thesis work.

The decision to command the moments about each of the three axes, instead of the single actuators, not only makes the system ASP, but also makes the controller independent of the operational conditions, i.e., which actuator is active at certain points of the trajectory. Indeed, the moments are assigned to the actuators outside the controller. Moreover, the maximum available moments differ along the trajectory depending on the flight 


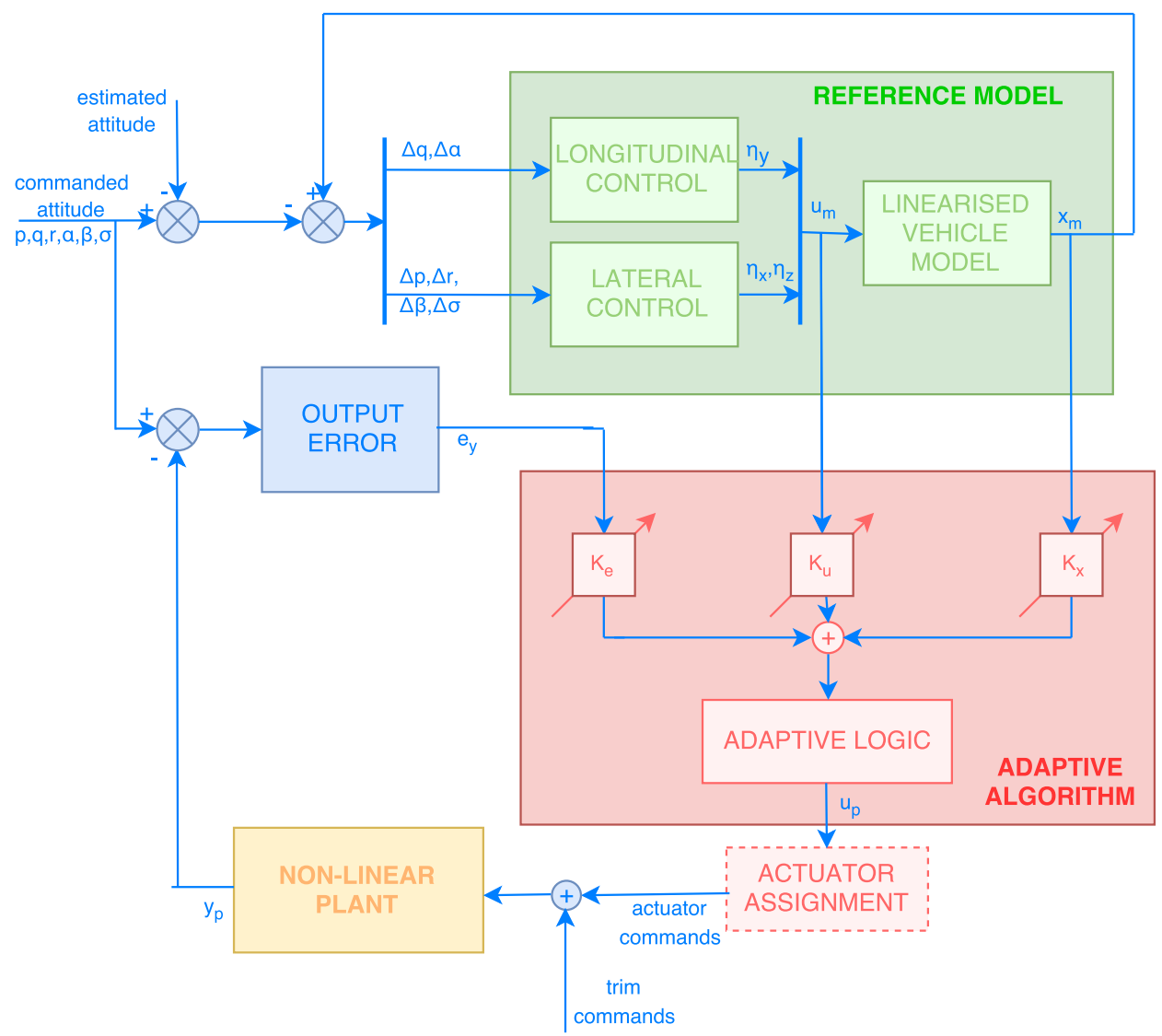

Figure 6.7: Schematic representation of the SAC system for FSS-1 attitude control.

conditions. So, to facilitate the design of the controller and to make this design valid for the entire mission, they are not the commanded moments that are outputted, but the moment fractions of the maximum available moments. The latter are identified with $\eta_{x}, \eta_{y}$, and $\eta_{z}$ and can vary between -1 and +1 . So, the adaptive controller is composed by three controllers, which are the roll, pitch, and yaw controllers. Each controller is characterised by a different output error $e_{y}$ (which is a scalar for each controller), model state vector $\mathbf{x}_{\mathbf{m}}$ and control vector $\mathbf{u}_{\mathbf{m}}$. In particular, for roll and yaw control, the weighting matrices $\mathbf{T}_{\mathbf{i}}$ and $\mathbf{T}_{\mathbf{p}}$ have dimensions $6 \times 6$ with $\mathbf{r}=\left(e_{x} p_{m} r_{m} \beta_{m} \sigma_{m} \eta_{x, m}\right)^{T}$ and $\mathbf{r}=\left(e_{z} p_{m} r_{m} \beta_{m} \sigma_{m} \eta_{z, m}\right)^{T}$, respectively. For pitch control, $\mathbf{T}_{\mathbf{i}}$ and $\mathbf{T}_{\mathbf{p}}$ have dimensions $4 \times 4$ with $\mathbf{r}=\left(\begin{array}{lll}e_{y} & q_{m} & \alpha_{m} \\ \eta_{y, m}\end{array}\right)^{T}$. These definitions help to show how the control output around an axis is directly related to the corresponding control error.

The general architecture of the SAC system is described in Figure 6.7. Three main elements can be distinguished: the reference model, the adaptive control, and the plant. The reference model is represented by the linearised state-space model of FSS-1 and the adaptive control algorithm is based on the definitions and logic described above. Details about the reference model and the adaptive controller design are given in the next sections. The plant represents the vehicle's flight dynamics, described by the non-linear model. The SAC architecture shall be linked to the software architecture of the entire system, which is shown in Figure B.1.

\subsubsection{Reference Model}

The reference model is a simplified representation of the actual system so that the performance specifications and requirements desired by the user can be more easily met. For this application, the reference model is represented by the LTI system of FSS-1, obtained by linearising the EoM around the (trimmed) reference trajectory (Eq.(4.74)).

The definition of the reference model is of extreme importance in the adaptive control system. Indeed, since it pursues the model following, a well designed reference model provides a more stable and robust performance. The reference model is stabilised by a simple, linear state-feedback controller. The moment-fraction LQR can be used to this purpose, thus the reference-model control design is fully detailed in Section 6.2.2. 


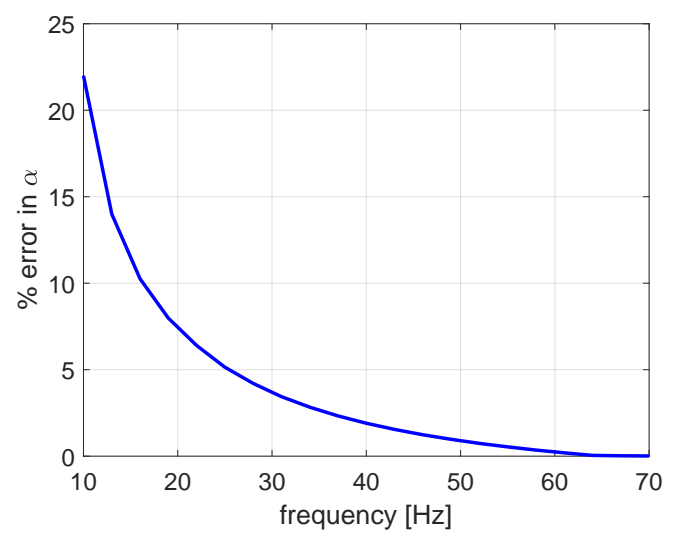

Figure 6.8: Influence of the reference-model sample frequency on the percentage error of $\alpha$.

The reference model and adaptive algorithm of the SAC system are digitised using a zero-order hold discretisation scheme. The sample frequency chosen for both the reference model and adaptive-gain algorithm can influence the performance. To identify the sample frequency of the reference-model dicretisation and update, the frequency of $65 \mathrm{~Hz}$ is taken as the baseline. Also for this analysis the response to an angle-of-attack step command along the re-entry trajectory $(M=15)$ is used. Figure 6.8 defines the relationship between the frequency of the reference model and the response error with respect to the reference value at $65 \mathrm{~Hz}$. It can be observed that the error has an exponential trend, so the lower the frequency the higher the error. It follows that for a global error of $1 \%$, a sampling frequency of $50 \mathrm{~Hz}$ should be used. Nonetheless, if a slightly higher divergence can be accepted, the frequency can be lowered, reducing the required simulation time. An error of $5 \%$ is obtained with a sample frequency of $25 \mathrm{~Hz}$. In conclusion, a baseline frequency between $20 \mathrm{and} 30 \mathrm{~Hz}$ should be chosen to ensure an accuracy of the reference-model propagator of about $5 \%$. A frequency of $25 \mathrm{~Hz}$ is selected to conduct the analyses of the present thesis work.

Mooij [2017] stated that, if the frequency of the controller is set equal to the one of the reference model, adequate performance is obtained. However, by keeping the reference model frequency at $25 \mathrm{~Hz}$, it is possible to reduce the SAC frequency up to $3 \mathrm{~Hz}$, but stable oscillations in the response and control effort are introduced. These oscillations can be avoided if the weighting matrices of the SAC algorithm are reduced of about 9099\% depending on the flight conditions, but at the expenses of a very slow transient response. So, it can be concluded that the design of the controller is linked to the frequency chosen for the SAC controller. Generally, the frequency of digital controllers is set up on the basis of the on-board computer characteristics and, thus, the frequency is increased only in case of degradation of the controller performance. Thus, the frequency of the controller is chosen to be equal to the one of the reference model, i.e., $25 \mathrm{~Hz}$. Moreover, it should be noticed that no requirements are provided to define the accuracy level that the SAC controller shall meet. So, to conduct the following studies it will be assumed that the required accuracy of the adaptive controller is $5 \%$ of the deviation of the plant state from the commanded nominal values.

\subsubsection{Baseline Controller Design}

Within the SAC controller, the reference model can be used based on two different methods. In the first option the model is used as part of the tracking system. When there is an error between the commanded attitude and the actual attitude, the model responds to this command by activating the control system with the purpose of bringing this deviation back to zero. When this is achieved, the command is realised and the reference model no longer provides a signal to the adaptive control algorithm. This method has been implemented by, for instance, Mooij [2002],[2004].

The second possibility is to define the output error as the difference between the guidance command and the plant state. This means that the guidance command directly activates the controller. Similarly to the first option, the model control finishes its actions once the guidance command is realised. However, the difference is that model state $\mathbf{x}_{\mathbf{m}}$ is non-zero. So, in presence of a guidance error or, in other words, when the output error $\mathbf{e}_{\mathbf{y}}$ starts deviating from zero, $\mathbf{x}_{\mathbf{m}}$ has a much larger corrective power than $\mathbf{e}_{\mathbf{y}}$. This can result in an improvement of the tracking performance.

Although the previous cited sources prove that the first option offers relatively good performance, in the current work both methods were implemented and analysed to investigate to what extent the second one im- 

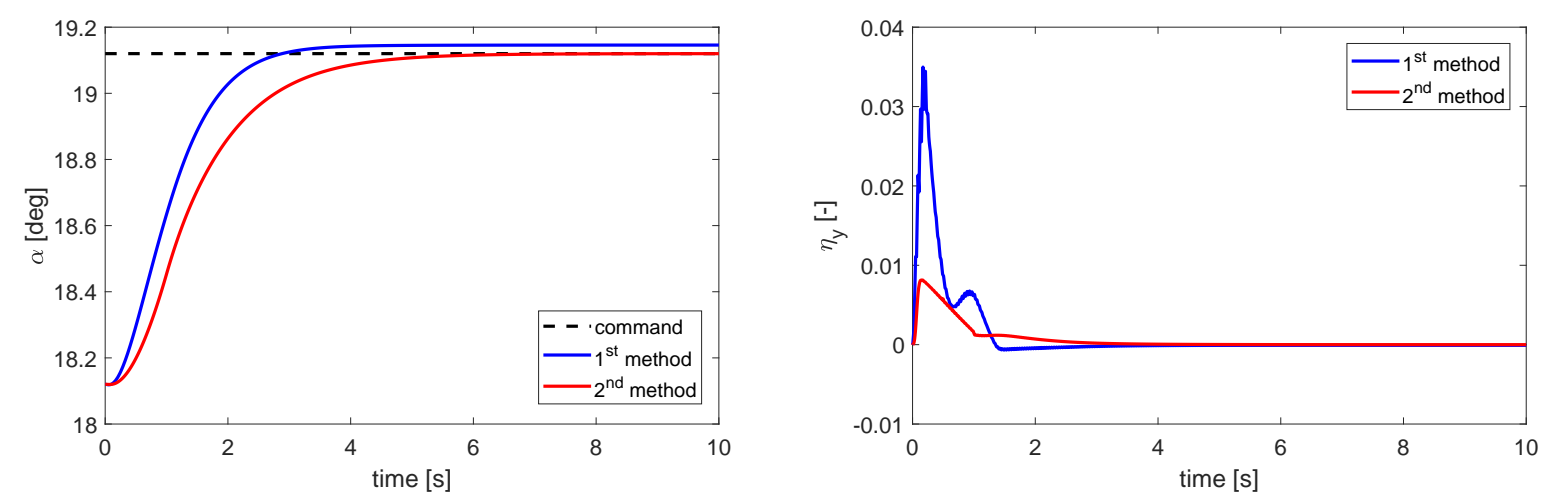

Figure 6.9: Difference in the system response to a step angle-of-attack command when the two methods of considering the reference model are used (results for $M=15$ along the re-entry).

proves the control performance. It was observed that, when an attitude command is provided, both the two methods are able to realise the command. As an example, the system response to a one-degree step command in the angle of attack is presented in Figure 6.9. There are actually some interesting differences between the two methods. For what concerns the response, it is quicker if using the first method with a settling time of about 3 $\mathrm{s}$ against $4.5 \mathrm{~s}$ with the second method. However, the latter reduces the final steady-state error and, from the control effort point of view, it requires a smaller control effort with reduced oscillations than the first one.

Furthermore, it was recognised that the second method makes it easier to design a controller that can be used at any flight condition and to respond to any kind of command. This means that, while the first method requires that the control-design parameters need to be set up and adjusted differently depending on the attitude command and on whether the ascent or the re-entry is flown, the second method necessitates to be set up just once and this design is generally valid for any attitude command and flight condition. This fact appears to be a crucial advantage, since one of the key requirement of a control system is that it should be suitable at any flight conditions and capable of realising any command even in presence of unforeseen disturbances. It should be noticed that, when the guidance error commanded is larger than $5^{\circ}$, the assumption of step command is no more appropriate. In fact, the impossibility to respond instantaneously to a command should be considered and a more relaxed response should be admitted. So, a ramp command is recommended in this case. In summary, by defining the control error as the deviation of the plant state from the guidance state command, the control system is easier to design and it results to be more reliable.

To design the SAC control system for the FSS-1, the weighting matrices are initially set so that the desired performance is obtained. However, to guarantee that this setting minimises the performance indices, an optimisation of the weighting matrices needs to be performed. Equations (6.39) and (6.40) define that the values of the weighing coefficients of $\mathbf{T}_{\mathbf{p}}$ and $\mathbf{T}_{\mathbf{i}}$ are related to the elements of $\mathbf{r}$. It was specified that these matrices should be designed in such a way that the constraint of positive (semi-)definiteness is respected. This condition is easily met when the weighting matrices are diagonal, while it becomes harder in presence of off-diagonal terms. At first, the weighting matrices are designed as diagonal matrices, later the influence of the off-diagonal elements is investigated.

At first, the weighting design parameters are set up so that a satisfactory response to angle-of-attack and bank-angle commands can be obtained. Afterwards, starting from this design, a Monte Carlo simulation $(N$ $=1000$ ) is executed to determine the optimal combination of design parameters. The performance of the response to an angle-of-attack step command is examined at $M=15$ along the ascent and re-entry trajectory. In both cases, the optimal combination of weighting coefficients allows for an improvement of the integrated pitch-moment fraction oscillation of about $55 \%$, while slightly improving the integrated control error and the integrated control effort with respect to the initial set of design parameters. Similarly, the controller is optimised to perform commands of the lateral motion. In the given example the performance indices are for the angle-of-attack command:

$$
\begin{aligned}
& \text { for ascent: } \sum_{\alpha_{e r r}}=278.9^{\circ} \mathrm{s} \quad \sum_{\eta_{y}}=14.4 \mathrm{~s} \quad \sum_{F_{\eta_{y}}}=0.3 \mathrm{~s} \\
& \text { for re-entry: } \sum_{\alpha_{e r r}}=266.1^{\circ} \mathrm{s} \quad \sum_{\eta_{y}}=3.0 \mathrm{~s} \quad \sum_{F_{\eta_{y}}}=0.4 \mathrm{~s}
\end{aligned}
$$


Table 6.1: Weighting matrix coefficient: baseline design.

\begin{tabular}{|c|c|c|c|c|c|c|c|c|}
\hline \multicolumn{9}{|c|}{ Baseline Design } \\
\hline \multicolumn{3}{|c|}{ Roll } & \multicolumn{3}{|c|}{ Pitch } & \multicolumn{3}{|c|}{ Yaw } \\
\hline Element & $\mathbf{T}_{\mathbf{p}, \mathbf{x}}$ & $\mathbf{T}_{\mathbf{i}, \mathbf{x}}$ & Element & $\mathbf{T}_{\mathbf{p}, \mathbf{y}}$ & $\mathbf{T}_{\mathbf{i}, \mathbf{y}}$ & Element & $\mathbf{T}_{\mathbf{p}, \mathbf{z}}$ & $\mathbf{T}_{\mathbf{i}, \mathbf{z}}$ \\
\hline$e_{y, x}$ & 13.5 & 22.9 & $e_{y, y}$ & 94.1 & 112.9 & $e_{y, z}$ & 79.1 & 77.7 \\
\hline$p_{m}$ & 6.23 & 42.1 & $q_{m}$ & 150.4 & 162.2 & $p_{m}$ & 156.9 & 176.9 \\
\hline$r_{m}$ & 24.5 & 76.2 & $\alpha_{m}$ & 57.8 & 572.7 & $r_{m}$ & 187.1 & 133.8 \\
\hline$\beta_{m}$ & 17.6 & 72.8 & $\eta_{y_{m}}$ & 1.3 & 3.6 & $\beta_{m}$ & 126.6 & 14.2 \\
\hline$\sigma_{m}$ & 6.6 & 564.2 & & & & $\sigma_{m}$ & 948.1 & 44.2 \\
\hline$\eta_{x_{m}}$ & 99.5 & 94.6 & & & & $\eta_{z_{m}}$ & 64.2 & 27.3 \\
\hline
\end{tabular}

and for the bank-angle command:

$$
\begin{aligned}
& \text { for ascent: }\left\{\begin{array}{lll}
\sum_{\sigma_{e r r}}=289.6^{\circ} \mathrm{s} & \sum_{\eta_{x}}=15.5 \mathrm{~s} & \sum_{\eta_{z}}=5.6 \mathrm{~s} \\
\sum_{F_{\eta_{x}}}=0.7 \mathrm{~s} & \sum_{F_{\eta_{z}}}=0.5 \mathrm{~s}
\end{array}\right. \\
& \text { for re-entry: }\left\{\begin{array}{lll}
\sum_{\sigma_{e r r}}=233.4^{\circ} \mathrm{s} & \sum_{\eta_{x}}=5.4 \mathrm{~s} & \sum_{\eta_{z}}=16.8 \mathrm{~s} \\
\sum_{F_{\eta_{x}}}=0.2 \mathrm{~s} & \sum_{F_{\eta_{z}}}=0.5 \mathrm{~s}
\end{array}\right.
\end{aligned}
$$

The identified control-system design is then applied to the other design points of the hypersonic trajectory, showing that it allows to improve the performance also in the other flight conditions. Also, to verify that the optimality of this design, a refined sensitivity analysis ( $\pm 10 \%$ variation of the design parameters) is computed around this design. From these analyses, the baseline control-system design is identified for an angle-of-attack and bank-angle step command. The design parameters of the baseline design are presented in Table 6.1, and the performance of this controller are shown in Figure 6.10 for the ascent trajectory and Figure 6.11 for the re-entry trajectory.

Although defining the matrices $\mathbf{T}_{\mathbf{p}}$ and $\mathbf{T}_{\mathbf{i}}$ as diagonal matrices provides quite an accurate response to a guidance command, we desire to investigate what impact the off-diagonal elements have on the performance. So, for the longitudinal controller the weighting matrices can now be defined as symmetric matrices as follows:

$$
\mathbf{T}=\left[\begin{array}{cccc}
T_{e_{y} e_{y}} & T_{e_{y} q_{m}} & T_{e_{y} \alpha_{m}} & T_{e_{y} M_{y}} \\
T_{q_{m} e_{y}} & T_{q_{m}} q_{m} & T_{q_{m} \alpha_{m}} & T_{q_{m} M_{y}} \\
T_{\alpha_{m} e_{y}} & T_{\alpha_{m} q_{m}} & T_{\alpha_{m} \alpha_{m}} & T_{\alpha_{m} M_{y}} \\
T_{M_{y} e_{y}} & T_{M_{y} q_{m}} & T_{M_{y} \alpha_{m}} & T_{M_{y y}}
\end{array}\right]
$$

A similar definition applies also for the lateral controller just by adapting them with the relative design variables. The influence of the off-diagonal elements can be studied by varying their values up to $100 \%$ of the corresponding diagonal value, while the diagonal terms are maintained at their fixed values identified before. In case of violation of the positive (semi-)definiteness constraint, the matrices are recalculating until the constraints are met. The results of the sensitivity analysis is presented in Figure 6.12 for the response to a step command in angle of attack in the point with $M=15$ along the re-entry trajectory. In case of non-zero offdiagonal elements of the weighting matrices, the performance is slightly improved by $2 \%$ in terms of integrated control error, at the expense of a marginally larger control effort and stronger oscillations of the elevator. It can be concluded that overall the presence of the off-diagonal elements in the design matrices does not significantly improve the performance of the controller. Thus, since the required performance can be obtained when the weighting matrices contain only diagonal elements, diagonal weighting matrices are preferred due to the much lower design effort that they require. Indeed, this allows for reducing the number of design parameters from 176 to 32 .

In the following of this report, the response to a one-degree step command in either $\alpha$ or $\sigma$ is analysed. This choice was driven by the fact that every command can be decomposed in a number of sequential unit steps. So, studying the response for a step command of one-degree provides conclusions that are valid for all the other commands. Figure 6.13 describes the plant response and the control effort required to realise an angleof-attack step command at the points of interests, i.e., where $M=5,10,15,20$, along the re-entry trajectory. Considering that all these points have the same active actuators, the behaviour of system depends only on the flight conditions, particularly on the Mach number and dynamic pressure. Along the re-entry trajectory, i.e., with decreasing Mach number and increasing dynamic pressure, the aerodynamic surfaces are more effective 

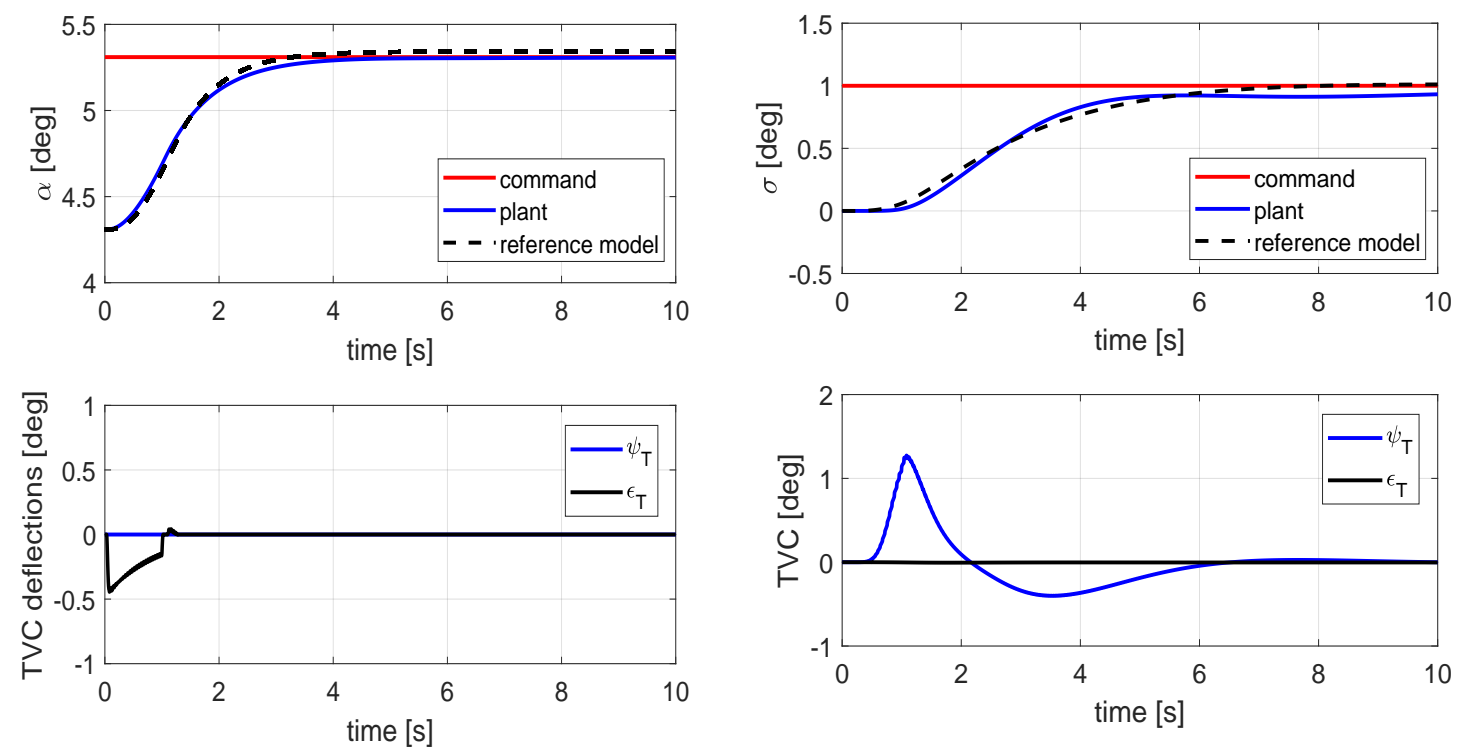

Figure 6.10: Baseline design: control error and control effort for an angle-of-attack (left) and bank-angle (right) step command at design point with $M=15$ along the ascent trajectory.
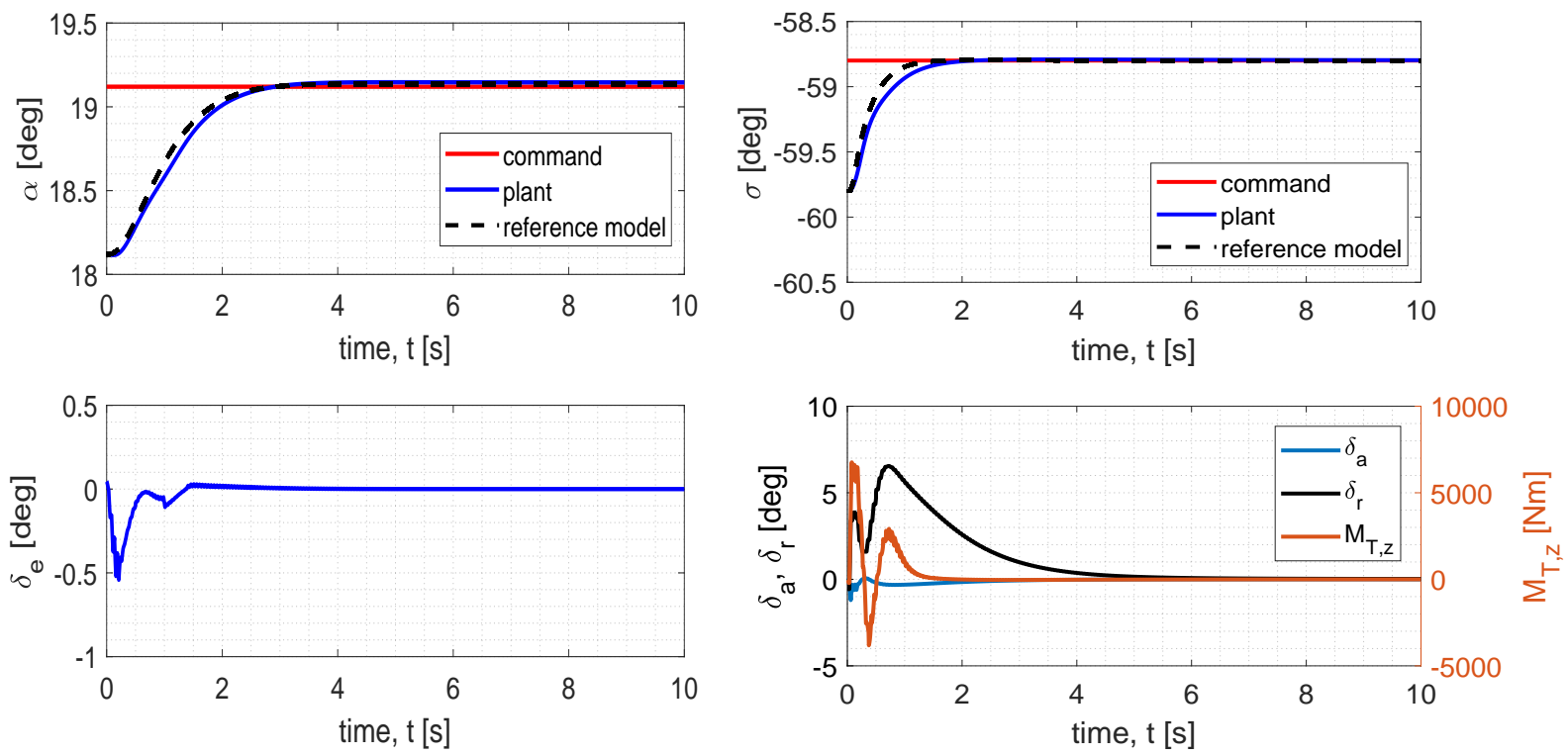

Figure 6.11: Baseline design: control error and control effort for an angle-of-attack (left) and bank-angle (right) step command at design point with $M=15$ along the re-entry trajectory.
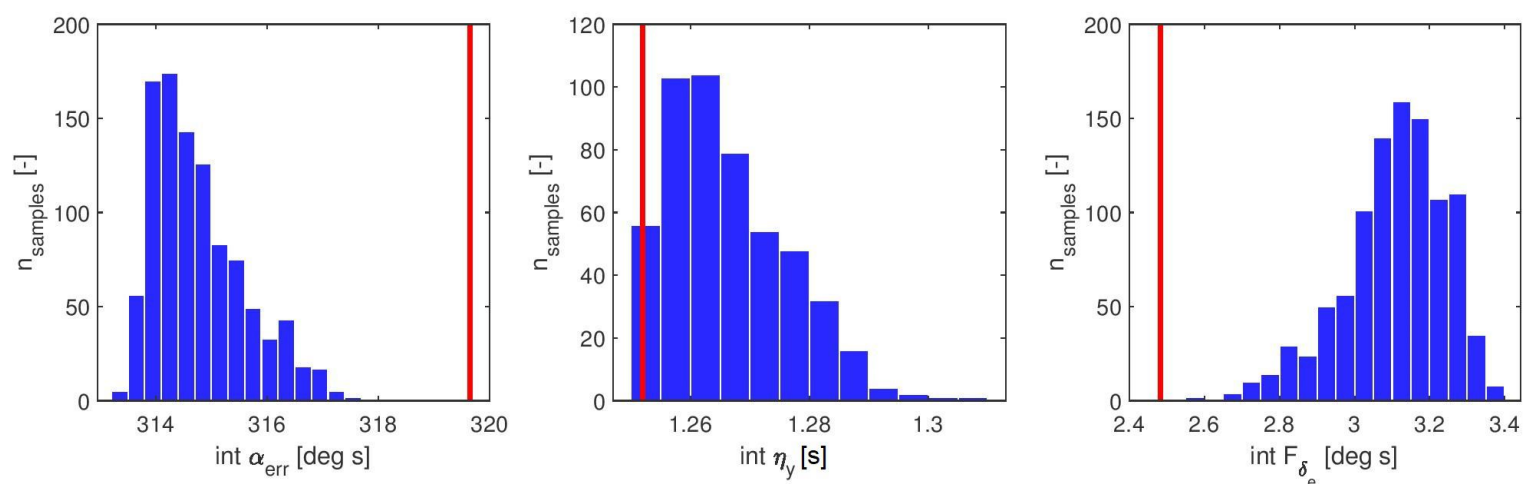

Figure 6.12: Non-zero off-diagonal elements of $T_{p}$ and $T_{i}$ : sensitivity of the response to an angle-of-attack step command in the point with $M=15$ along the re-entry trajectory. 

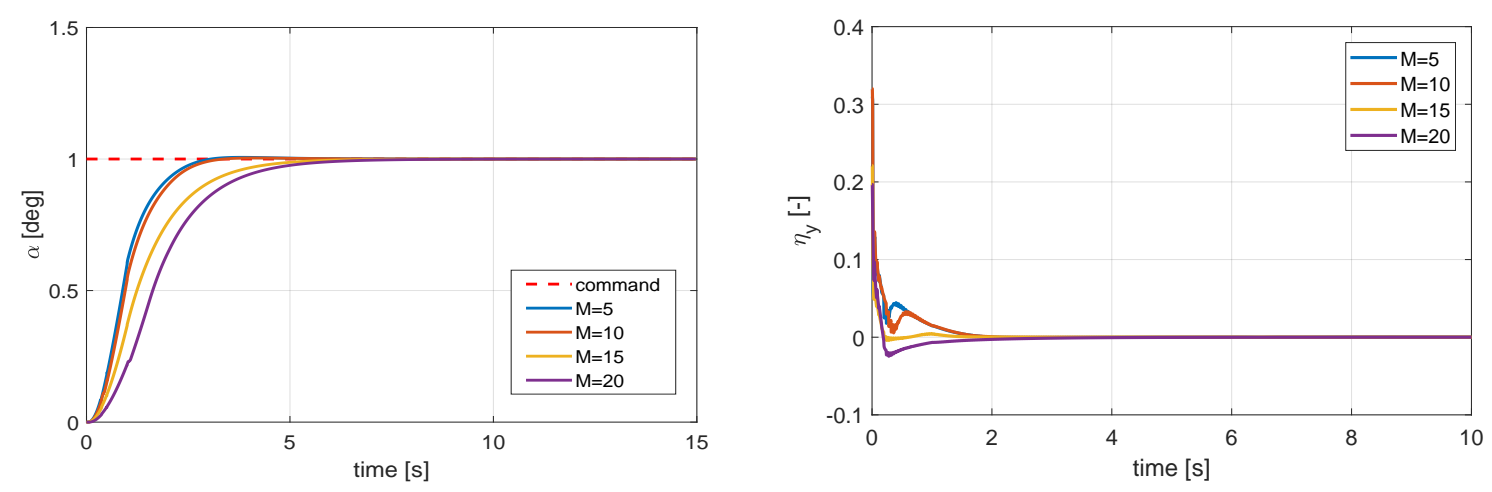

Figure 6.13: Angle-of-attack step responses of the plant at the points of interest along the re-entry trajectory.

resulting in a more rapid response, while requiring a slightly larger control effort. The same is observed for a bank-angle step command. The same behaviour cannot be noticed in the corresponding characteristic points along the ascent flight. The reason behind it is that, since TVC is used to control the vehicle along the ascent, its effectiveness remains quite high due to the high thrust magnitude, independently of the decreasing dynamic pressure. Thus, it offers almost the same control power at all the design points, which means that no significant difference can be noticed in the control response and effort.

In Section 7.2, robust control-system design of the SAC controller is identified starting from the baseline control design. Afterwards, the robust controller is used to conduct the controllability analyses and examine the response to atmospheric disturbances.

\subsection{Actuator Assignment}

The actuator assignment is a procedure that, on the basis of the commanded moment fractions, allocates the commanded control moments about $x-, y_{-}$, and $z$-axes to the active actuators. This allows for simplifing the SAC implementation and consistently improve its performance. The theory presented in the remaining of this section is taken from Brinkman [2017] and Wu et al. [2000].

The actuator assignment algorithm was applied by Wu et al. [2000] to calculate, starting from the moment fractions, the control-surface deflections and thruster moments to control the X-38 along its re-entry flight. For this purpose, a linearised aerodynamics is used so that the control-surfaces deflections can be determined by means of a simple inversion of the aerodynamics. However, being the aerodynamics mainly characterised by non-linear functions, this procedure leads to an error of 6\%. Also, Durham [1993] defined a control-allocation algorithm based on a bisecting edge-searching method to distribute the control moments to the active actuators of the F-15 Advanced Control Technology for Integrated VEhicles (ACTIVE). Also in this case, the linearisation of the aerodynamics is required, inducing an analogous percentage of error. A similar method is followed in this thesis project but, to avoid to use a linearised aerodynamics and get rid of the induced error, the flatness property of the aerodynamic database (described in Section 2.1.1) will be exploited. This property allows to obtain the actuator deflection angles from an inverse linear interpolation applied to the data tables of the aerodynamic coefficients.

Once the moment fractions are commanded by the controller, the commanded moments can be computed by simply multiplying the moment fractions for the maximum available moments. The commanded moments need to be allocated over the actuators. The adopted method requires that along the re-entry trajectory the RCS thrusters are used only when the control surfaces are not able to provide the whole commanded moments. We are aware that this can eventually lead to the saturation of the control surfaces at low dynamic pressure and that some alternatives can be considered. For instance, the maximum deflections can be linearly or quadratically decreased at decreasing dynamic pressure, but it is considered beyond the scope of this thesis work and left for further research. Along the ascent trajectory the control surfaces are not able to compute the commands for $\bar{q}<5000 \mathrm{~Pa}$. This condition is encountered for Mach number larger than 5, thus TVC becomes essential. It follows that, for $M>5$ the actuator assignment has to directly allocate the commanded moments to the TVC deflections only. This is obtained by setting the maximum control surface deflections to zero for $M>5$ along the ascent trajectory. In the following of this section, the description of the actuator assignment is presented in its complete form (i.e., commanded moments provided by both aerodynamic surface deflections and propul- 

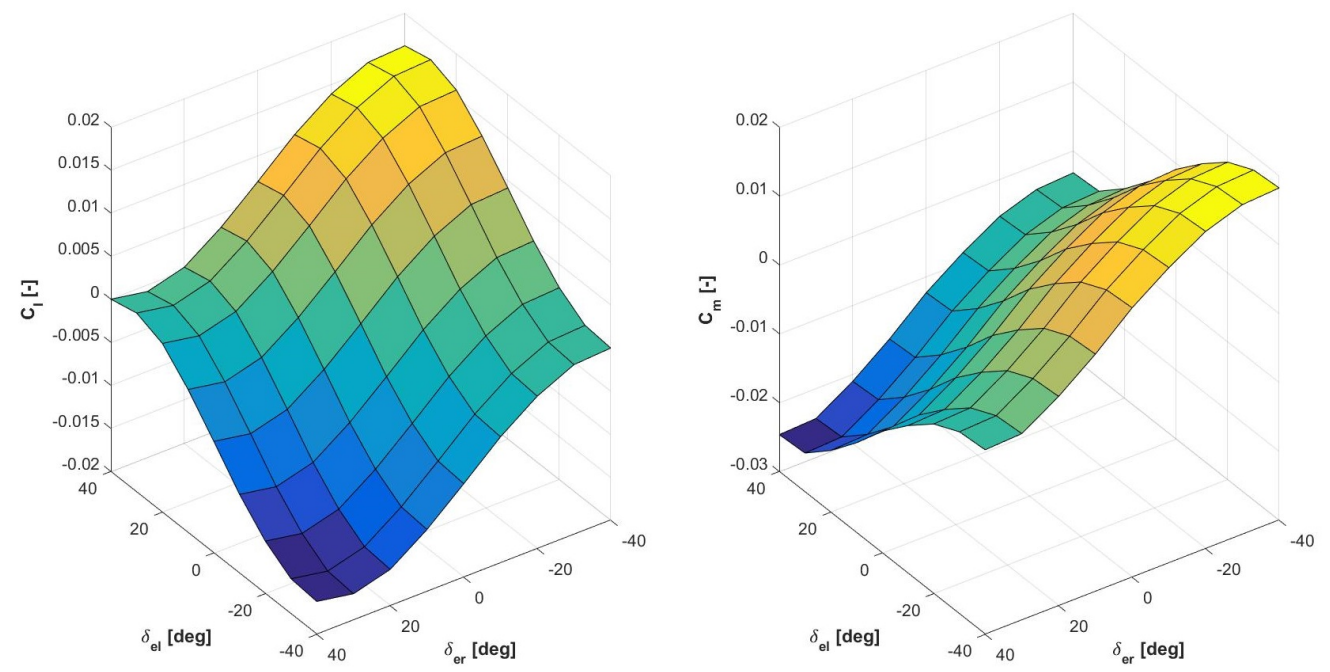

Figure 6.14: Roll (left) and pitch (right) moment coefficients as function of the elevons deflections, $\delta_{w, l}$ and $\delta_{w, r}$, which correspond to $\delta_{e, l}$ and $\delta_{e, r}$ in figure (calculated at $M=17$ along the HORUS re-entry trajectory) [Mooij, 2018]

sive moments). However, it should be noted that for $M>5$ during the ascent, the procedure is simplified by setting the aerodynamic moments to zero so that the TVC moments simply equate the total commanded moments.

The required actual moments can be computed:

$$
\begin{aligned}
& \mathscr{L}_{\text {aero }}=\operatorname{sign}\left(\eta_{x}\right) \min \left(\left\|\mathscr{L}_{\text {cmd }}\right\|, \mathscr{L}_{\text {aero }} \text { max }\right) \\
& \mathscr{M}_{\text {aero }}=\operatorname{sign}\left(\eta_{y}\right) \min \left(\left\|\mathscr{M}_{\text {cmd }}\right\|, \mathscr{M}_{\text {aer } o_{\text {max }}}\right) \\
& \mathscr{N}_{\text {aero }}=\operatorname{sign}\left(\eta_{z}\right) \min \left(\left\|\mathscr{N}_{\text {cmd }}\right\|, \mathscr{N}_{\text {aero }}{ }_{\text {max }}\right) \\
& M_{T_{x, 0}}=\mathscr{L}_{c m d}-\mathscr{L}_{\text {aero }} \\
& M_{T_{y, 0}}=\mathscr{M}_{c m d}-\mathscr{M}_{\text {aero }} \\
& M_{T_{z, 0}}=\mathscr{N}_{\text {cmd }}-\mathscr{N}_{\text {aero }}
\end{aligned}
$$

where the subscript "0" is added to indicate the nominal commanded thruster moments and to differentiate them from the corrective thruster moments, which are introduced below. From these expressions the required aerodynamic coefficients can be defined:

$$
C_{l_{c m d}}=\frac{\mathscr{L}_{\text {aero }}}{\bar{q} S_{\text {ref }} b_{\text {ref }}}, \quad C_{m_{\text {cmd }}}=\frac{\mathscr{M}_{\text {aero }}}{\bar{q} S_{\text {ref }} c_{\text {ref }}}, \quad C_{n_{\text {cmd }}}=\frac{\mathscr{N}_{\text {aero }}}{\bar{q} S_{\text {ref }} b_{\text {ref }}}
$$

from which the control-surface deflections can be calculated. It should be noticed that the elevons, combining the elevator and aileron functions, have to provide both the roll and pitch moments. Their contribution to the roll and pitch coefficients can be represented as a three-dimensional surface with two independent variables, $\delta_{w, l}$ and $\delta_{w, r}$, as shown in Figure 6.14. Thus, the wing-flap deflection angles can be defined as the intersection point of the surfaces $C_{l_{\delta}}-C_{l_{c m d}}$ and $C_{m_{\delta}}-C_{m_{c m d}}$ and the XY-plane, since this is where the deflection angles provides both the commanded roll and pitch coefficients. This means mathematically that the solution space $\delta$ is defined as:

$$
\delta=\underset{\delta_{w, r}, \delta_{w, l}}{\arg }\left(C_{l_{\delta}}-C_{l_{c m d}}\right) \cap\left(C_{m_{\delta}}-C_{m_{c m d}}\right) \cap(X Y)
$$

where $\cap$ is the intersection operator. In case of multiple solutions of $\delta_{w, l}$ and $\delta_{w, r}$, the values closest to the current ones are chosen to reduce the oscillatory behaviour. The algorithm to define the solution $\delta$ is explained in detail in Brinkman [2017].

Once the elevon deflections are calculated, the rudder deflection needs to be defined. Since the elevons can also contribute to the yaw moments, a corrected yaw moment coefficients is determined:

$$
C_{n_{c m d, c o r r}}=C_{n_{c m d}}-\Delta C_{n_{w, l}}-\Delta C_{n_{w, r}}
$$

from which the left and right rudder deflections can be determined. For mechanical constraints, one rudder at a time can be activated and it can move only outward. Thus, for $C_{n_{c m d, c o r r}}<0$ the left rudder is active, 
while for $C_{n_{\text {cmd,corr }}}>$ the right rudder is active. Thus, the corresponding deflection angles can be calculated. If $C_{n_{\text {cmd,corr }}}=0$, no rudders are activated.

The active thrusters play a key role in case the control surfaces get saturated and they cannot provide the whole commanded moment coefficients. Moreover, it can also happen that a solution of elevon deflections that realise both the required roll and pitch coefficients does not exist and thus an error between the commanded and achievable moments is present. In these cases, the thrusters can be used to support the control surfaces in achieving the desired moments.

The corrective thruster coefficients can be computed by means of an updated prediction of the aerodynamic moments, which can be done with an on-board implementation of the aerodynamic database. Thus, we get:

$$
\begin{aligned}
& C_{T_{x, \text { corr }}}=C_{l_{c m d}}-\Delta C_{l_{e, l}}-\Delta C_{l_{e, r}} \\
& C_{T_{y, \text { corr }}}=C_{m_{c m d}}-\Delta C_{m_{e, l}}-\Delta C_{m_{e, r}} \\
& C_{T_{z, \text { corr }}}=C_{n_{c m d}}-\Delta C_{n_{e, l}}-\Delta C_{n_{e, r}}-\Delta C_{n_{r, l}}-\Delta C_{n_{r, r}}-\left[\Delta\left(\frac{\partial C_{n}}{\partial \beta}\right)_{r, l}+\Delta\left(\frac{\partial C_{n}}{\partial \beta}\right)_{r, r}\right] \beta
\end{aligned}
$$

where the perturbing rudder moments in case of non-zero sideslip angle is considered. Finally, the commanded thruster moments can be defined as the summation of the moments induced by the nominal commanded thruster moments and the corrected thruster coefficient, as follows:

$$
\begin{aligned}
& M_{T_{x, \text { cmd }}}=M_{T_{x, 0}}+C_{T_{x, \text { corr }}} \bar{q} S_{r e f} b_{r e f} \\
& M_{T_{y, \text { cmd }}}=M_{T_{y, 0}}+C_{T_{y, \text { corr }}} \bar{q} S_{r e f} c_{r e f} \\
& M_{T_{z, \text { cmd }}}=M_{T_{z, 0}}+C_{T_{z, \text { corr }}} \bar{q} S_{r e f} b_{r e f}
\end{aligned}
$$




\section{Robust Design \& Controllability}

The control system is designed to control the vehicle along its reference trajectory, even if deviations from the nominal conditions can occur due to uncertainties in initial conditions, atmospheric status and vehicle parameters. Thus, it is desirable that the control system designed for FSS-1 is able to control the vehicle and realise the input commands even in presence of design uncertainties. Although these perturbations cannot be predicted, the control system has to be able to minimize the effects of these perturbations on the system so that the nominal trajectory can be flown, guaranteeing the mission success. Thus, the control system should be robust with respect to disturbances, which means it should be as insensitive to disturbances as possible without eliminating the source of the disturbances. Section 7.1 presents the theory of the well-known robust design technique. This theory is applied to improve the robustness of the SAC controller. The sensitivity analysis is set up and conducted in Section 7.2 so that the robust design is identified and its properties are described.

Once the control system is designed, verified (see Appendix B.2) and integrated into the system, the controllability analyses can be performed. The controllability characteristics of both longitudinal and lateraldirectional motion are investigated in Section 7.3. Finally, Section 7.4 explores the capability of the system in response to atmospheric disturbances, in particular: steady-state wind, wind gusts and turbulence.

\subsection{Robust Design Methodology}

To obtain a robust control system, a robust design methodology is applied, which is a technique defined by Phadke [1989] as an "engineering methodology for improving productivity during research and development so that high-quality products can be produced quickly and at low cost". The concept behind robust design is to improve the quality of the product by minimising the effect of the cause of variation without eliminating the cause. The response of the control system can be influenced by three classes of parameters:

- signal factors: parameters set by the user, expressing the intended value for the product's response;

- noise factors: parameters that cannot be controlled by the designer; they can be external (environmental and load factors), manufacturing non-uniformity or wear-out and process drift;

- control factors: parameters that can be specified freely by the designer.

One of the major tools that can be exploit in robust design is the signal-to-noise ratio (SNR), which is a measure of the quality of the product. The SNR is measured in decibels $(\mathrm{dB})$ and it is defined as follows:

$$
\eta=10 \log _{10}\left(\frac{\bar{y}^{2}}{\sigma^{2}}\right)
$$

with $\bar{y}$ being the mean value of the response or, in other words, the desirable value, and $\sigma$ being the standard deviation that quantifies the effect of the noise factors. The most robust design is the one characterised by the maximum value of SNR, which corresponds to minimising the sensitivity to noise factors, by either increasing the nominal performance $\bar{y}$, or by reducing the standard deviation $\sigma$. To study the robustness of the control system of the FSS-1, the SNR of the performance metrics of the integrated control error and effort, described in Section 6.1.1, will be exploited.

In Section 6.3.3 the control design parameters are optimised for the nominal flight conditions, defining the baseline controller design. To identify the robust design of the SAC control system for the FSS-1 reference 


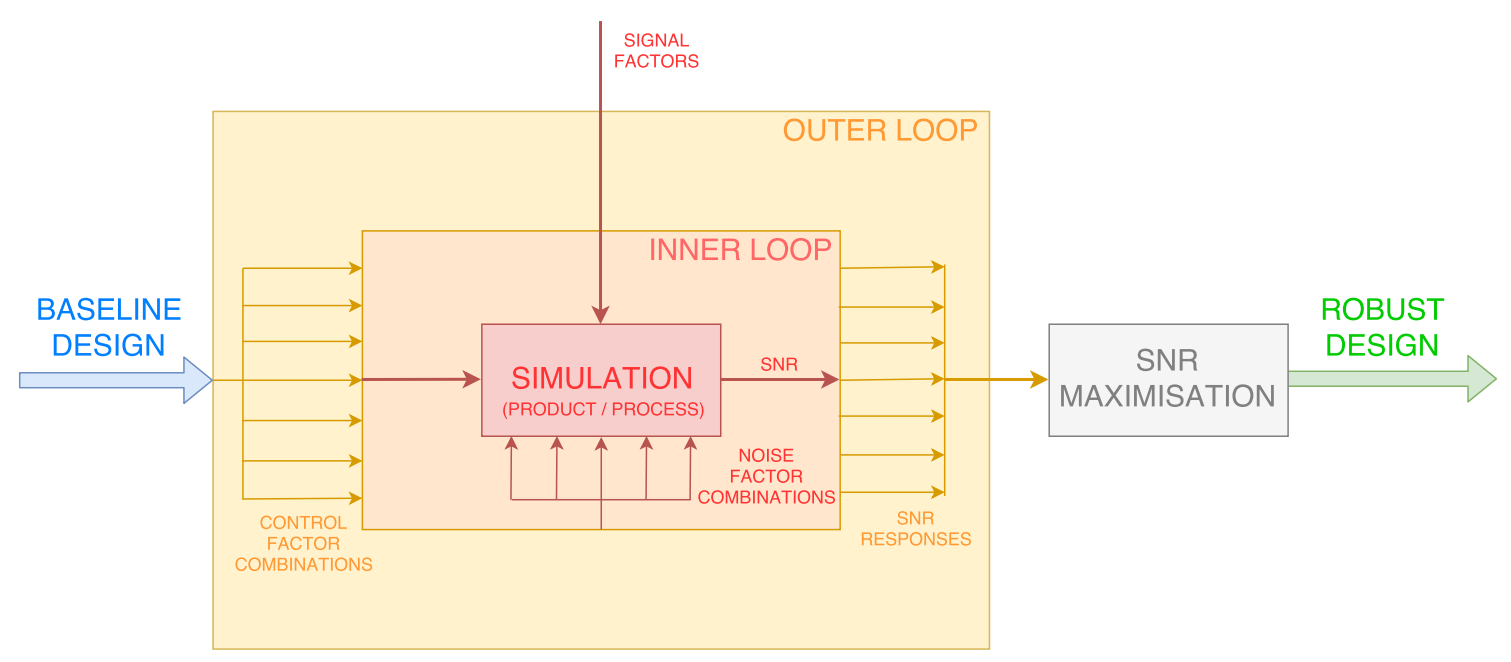

Figure 7.1: Schematic representation of the robust design process.

vehicle, a double-loop sensitivity analysis is designed. A schematic representation of the robust design process is illustrated in Figure 7.1. The sensitivity analyses involve two design loops: in the outer loop the control factors are varied around the baseline design, while in the inner loop the noise factors are varied. Thus, for each control-factor combination identified in the outer loop, a sensitivity analysis is conducted in the inner loop for all the noise-factor combinations, with a signal-to-noise ratio as outcome. At the end of the simulation, a number of signal-to-noise ratios equal to the number of outer-loop combinations is available. The robust design results to be the combination of control design parameters that is characterised by the maximum signalto-noise ratio.

\subsection{Robust Control-System Design}

The robust design technique is applied to identify the robust control-system design for the reference mission to ensure that the controller is as insensitive as possible to uncertainties and disturbances of the system. In this analysis the baseline design of the controller, identified in Section 6.3.3, is used as reference to compare the results of the different control designs obtained thought the sensitivity analysis.

As mentioned before, the robust design technique requires that the weighting matrix coefficients are varied in the outer loop. It is possible that the robust design is different from the optimal baseline design, thus the variation of these control factors should be sufficient to investigate all the possible combinations. On the other hand, we would like that the robust design does not move away too much from the optimal design because it is preferable that it continues to ensure minimal control error and effort. To this end, it follows that a range of $\pm 50 \%$ variation of the weighting matrix coefficients is suitable.

The noise factors are varied in the inner loop with respect to their nominal values. They are:

- initial conditions: $p_{0}, r_{0}, q_{0}, \alpha_{0}, \beta_{0}, \sigma_{0}$

- mass and inertia properties: $m, \mathbf{I}$

- atmospheric properties: $\rho$

- aerodynamic coefficients: $C_{D}, C_{S}, C_{L}, C_{l}, C_{m}, C_{n}$.

To identify the possible level of uncertanty that could interests these parameters, we should remember that the system we are working with is identified from the documentation by Daimler-Benz Aerospace [1998], from which the initial conditions, mass, inertia, and atmospheric properties are calculated graphically. Moreover, the aerodynamic coefficients are obtained by adjusting the HORUS aerodynamic database. It follows that the uncertainty of the noise factors could be quite high. For this reason, it is assumed that the noise factors can be subjected to perturbation from $\pm 10 \%$ to $\pm 50 \%$ of their nominal value, to consider both the best case and worst case scenario.

The sensitivity analysis is implemented so that $N=500$ simulations for outer loop and $M=500$ for the inner loop, for a total of 250,000 simulations. The results are generated by initializing the random number generator (RNG) with the same seed at every simulation batch, so that it is possible to reproduce the noise 

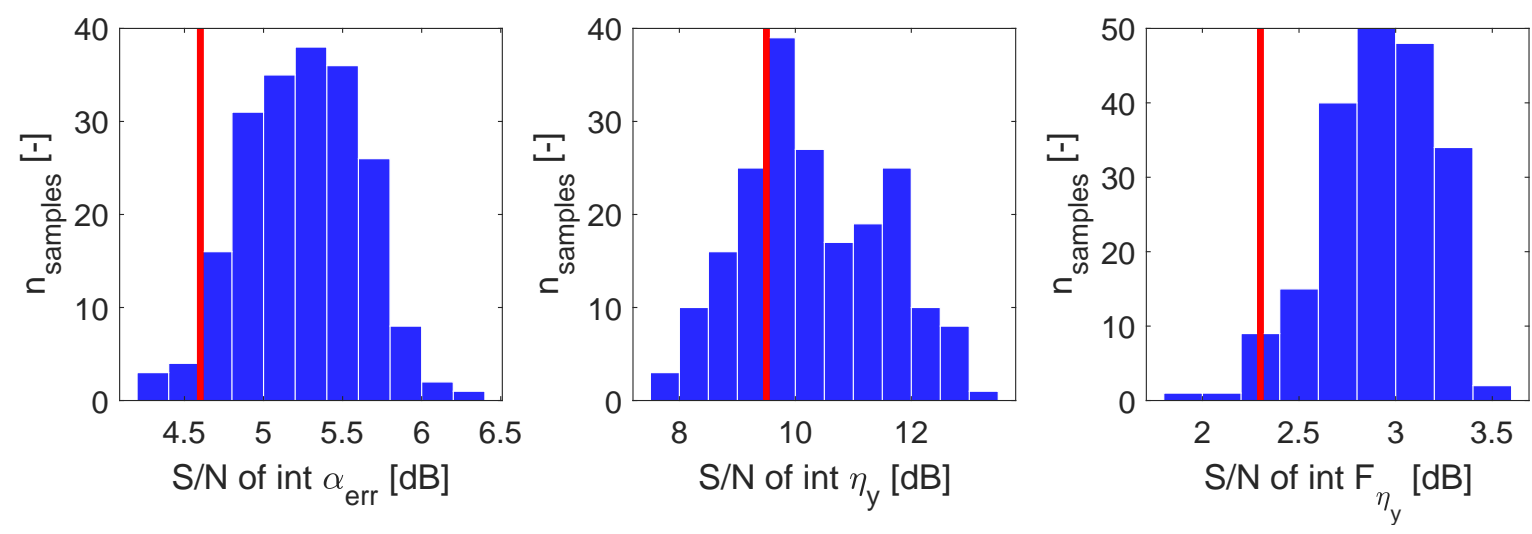

Figure 7.2: SNR of the performance indices due to uniform variation ( $\pm 50 \%$ ) of the noise factors to realise a step angle-of-attack command at design point with $M=15$ of the re-entry trajectory. The red line represents the baseline design.

factor variations for each combination of control factors. In this way, it can be ensured that the design space is entirely covered and it is possible to obtain statistically relevant results. Also, two batches of simulations are performed: one for $\pm 10 \%$ and one for $\pm 50 \%$ variation of the noise factors from their nominal value. The most robust combination of weighting matrix coefficients is finally identified as the one that maximises the signal-to-noise ratios that are evaluated for each performance indices of the integrated control effort and error.

\subsubsection{Longitudinal Motion}

The results show that a possible improvement in terms of robustness is possible with respect to the optimal baseline design. In particular, both during the ascent and re-entry, it is revealed that a combination of design parameters is found to be robust with respect to both moderate uncertainties and severe uncertainties of the noise factors, so that the controller is able to realise the commands even in the worst case scenario of perturbations. Indeed, the same weighting coefficient combination maximises the SNR of the performance indices in the both batches of simulations with $\pm 10 \%$ and $\pm 50 \%$ variation of the noise factors.

The histograms of the batch of simulations with $\pm 50 \%$ variation of the noise factors is presented in Figure 7.2 for the re-entry flight. An analogous behaviour is obtained for the ascent flight. Indeed, in both cases it is possible to notice that, although the noise factors are varied significantly, the variation of SNR of the performance indices is not so large. However, an improvement in terms of robustness is still possible.

It should be said that the SNR maximisation can be provided, for the different performance indices, by different combinations of design parameters. Thus, it is necessary to ponder on what are the SNR of the performance indices that allow for a larger margin of improvement and, finally, propose a trade-off so that system overall results as insensitive to disturbances as possible. The robust weighting coefficients and results are presented in Tables 7.1 and 7.2. The tables shows that the robust design maximises the SNR of the performance parameters with respect to those of the baseline design, while still maintaining excellent values of integrated error and effort.

\subsubsection{Lateral Motion}

Similarly, the results of the sensitivity analysis for the lateral controller present that, for moderate disturbances $( \pm 10 \%)$ and severe disturbances $( \pm 50 \%)$, a robust combination of design parameters can be identified.

For the lateral motion, the histograms of the worst case scenario are presented in Figures 7.3 for the ascent trajectory. The robust weighting coefficients and results are presented in Tables 7.1 and 7.2. The table presents the values of the SNR of the performance indices, which are maximised with respect to those of the optimal design, while showing still a good performance in terms of integrated control error and effort. A considerable improvement of the SNR values is obtained for almost all the performance indices, apart from the integrated control error that is slightly lower.

It should be noticed that the parameters that, during the robustness analysis for both the longitudinal and lateral motion, showed a larger margin of improvements were the SNR of the integrated oscillations of the actuators, both in the re-entry and ascent trajectory. It should be noticed that the performance of the controller remains similar to the one of the baseline controller in nominal conditions, i.e., when no perturbations are 

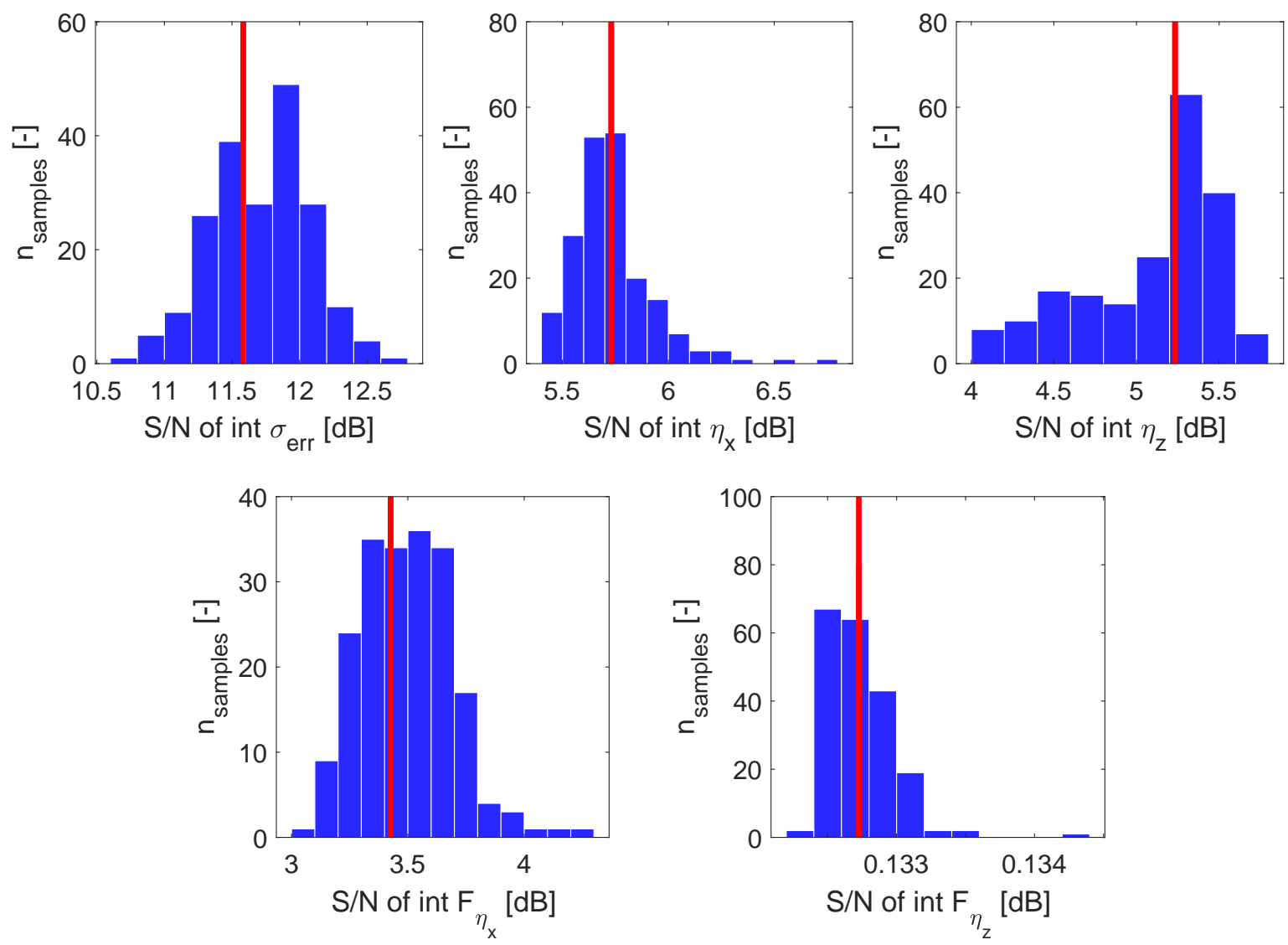

Figure 7.3: Signal-to-noise ratio of the performance indices due to uniform variation $( \pm 50 \%)$ of the noise factors to realise a step bank-angle command in the point with $M=15$ of the ascent trajectory. The red line represents the baseline design.

Table 7.1: Weighting matrix coefficient: baseline design.

\begin{tabular}{|c|c|c|c|c|c|c|c|c|}
\hline \multicolumn{9}{|c|}{ Robust Design } \\
\hline \multicolumn{3}{|c|}{ Roll } & \multicolumn{3}{|c|}{ Pitch } & \multicolumn{3}{|c|}{ Yaw } \\
\hline Element & $\mathbf{T}_{\mathbf{p}, \mathbf{x}}$ & $\mathbf{T}_{\mathbf{i}, \mathbf{x}}$ & Element & $\mathbf{T}_{\mathbf{p}, \mathbf{y}}$ & $\mathbf{T}_{\mathbf{i}, \mathbf{y}}$ & Element & $\mathbf{T}_{\mathbf{p}, \mathbf{z}}$ & $\mathbf{T}_{\mathbf{i}, \mathbf{z}}$ \\
\hline$e_{y, x}$ & 62.85 & 72.19 & $e_{y, y}$ & 106.4 & 162.6 & $e_{y, z}$ & 53.1 & 28.3 \\
\hline$p_{m}$ & 143.0 & 104.6 & $q_{m}$ & 221.9 & 150.8 & $p_{m}$ & 50.4 & 91.7 \\
\hline$r_{m}$ & 142.36 & 62.6 & $\alpha_{m}$ & 68.0 & 816.5 & $r_{m}$ & 62.4 & 72.1 \\
\hline$\beta_{m}$ & 126.4 & 71.3 & $\eta_{y_{m}}$ & 1.9 & 5.4 & $\beta_{m}$ & 114.6 & 88.9 \\
\hline$\sigma_{m}$ & 104.5 & 517.3 & & & & $\sigma_{m}$ & 354.7 & 61.5 \\
\hline$\eta_{x_{m}}$ & 144.2 & 74.6 & & & & $\eta_{z_{m}}$ & 51.1 & 55.1 \\
\hline
\end{tabular}

applied. However, the maximisation of the SNR makes the controller more robust to disturbances. This represents a pleasing outcome of the robustness analysis, since it was possible to obtain a design that, not only is as insensitive as possible to noise factors, but also maintains satisfactory values of the performance indices. As a final note, comparing the baseline design with the robust design shows that, overall, the coefficients that are increased more are those relatives to $\mathbf{e}_{\mathbf{y}}, \alpha_{m}, \sigma_{m}$, and $\boldsymbol{\eta}_{\boldsymbol{m}}$. The reason behind this can be found in the key principle of the adaptive control system. This is the model-following scheme, for which the plant should realise the command by following the response of the model and activating the actuators as similar as possible to what the model does.

In the following sections, the performance of the controller are studied to examine if it is adequate to stabilise the vehicle on the basis of what is specified in the MIL-documents. Also, a comparison of the performance of the robust and baseline controller in case of uncertainties will be studied. 
Table 7.2: Performance indices and signal-to-noise ratios calculated at point $M=15$ along the re-entry trajectory: comparison between baseline design and robust design.

\begin{tabular}{|c|c|c|c|c|c|}
\hline \multicolumn{3}{|c|}{ Longitudinal Motion } & \multicolumn{3}{|c|}{ Lateral Motion } \\
\hline Perf. Indices & Baseline Design & Robust Design & Perf. Indices & Baseline Design & Robust Design \\
\hline$\sum_{\alpha_{e r r}}$ & $266.1^{\circ} \mathrm{s}$ & $340.3^{\circ} \mathrm{s}$ & $\sum_{\sigma_{e r r}}$ & $233.4^{\circ} \mathrm{s}$ & $237.2^{\circ} \mathrm{s}$ \\
\hline$\sum_{\eta_{y}}$ & $3.0 \mathrm{~s}$ & $1.9 \mathrm{~s}$ & $\sum_{\eta_{x}}$ & $5.4 \mathrm{~s}$ & $5.2 \mathrm{~s}$ \\
\hline \multirow[t]{3}{*}{$\sum_{F_{\eta_{y}}}$} & $0.4 \mathrm{~s}$ & $0.6 \mathrm{~s}$ & $\sum_{\eta_{z}}$ & $16.8 \mathrm{~s}$ & $16.9 \mathrm{~s}$ \\
\hline & & & $\sum_{F_{\eta_{x}}}$ & $0.2 \mathrm{~s}$ & $0.3 \mathrm{~s}$ \\
\hline & & & $\sum_{F_{\eta_{z}}}$ & $0.5 \mathrm{~s}$ & $0.5 \mathrm{~s}$ \\
\hline SNR & Baseline Design & Robust Design & SNR & Baseline Design & Robust Design \\
\hline$\eta_{\sum_{\alpha_{e r r}}}$ & $4.5 \mathrm{~dB}$ & $5.3 \mathrm{~dB}$ & $\eta_{\sum_{\sigma_{e r r}}}$ & $1.9 \mathrm{~dB}$ & $2.5 \mathrm{~dB}$ \\
\hline$\eta_{\sum_{\eta_{y}}}$ & $9.5 \mathrm{~dB}$ & $12.8 \mathrm{~dB}$ & $\eta_{\sum_{\eta_{x}}}$ & $6.2 \mathrm{~dB}$ & $7.3 \mathrm{~dB}$ \\
\hline \multirow[t]{3}{*}{$\eta_{\sum_{F_{\eta}}}$} & $2.3 \mathrm{~dB}$ & $3.5 \mathrm{~dB}$ & $\eta_{\sum_{\eta_{z}}}$ & $2.6 \mathrm{~dB}$ & $3.9 \mathrm{~dB}$ \\
\hline & & & $\eta_{\sum_{F_{\eta_{x}}}}$ & $1.7 \mathrm{~dB}$ & $3.2 \mathrm{~dB}$ \\
\hline & & & $\eta_{\sum_{F_{\eta_{z}}}}$ & $0.3 \mathrm{~dB}$ & $0.5 \mathrm{~dB}$ \\
\hline
\end{tabular}
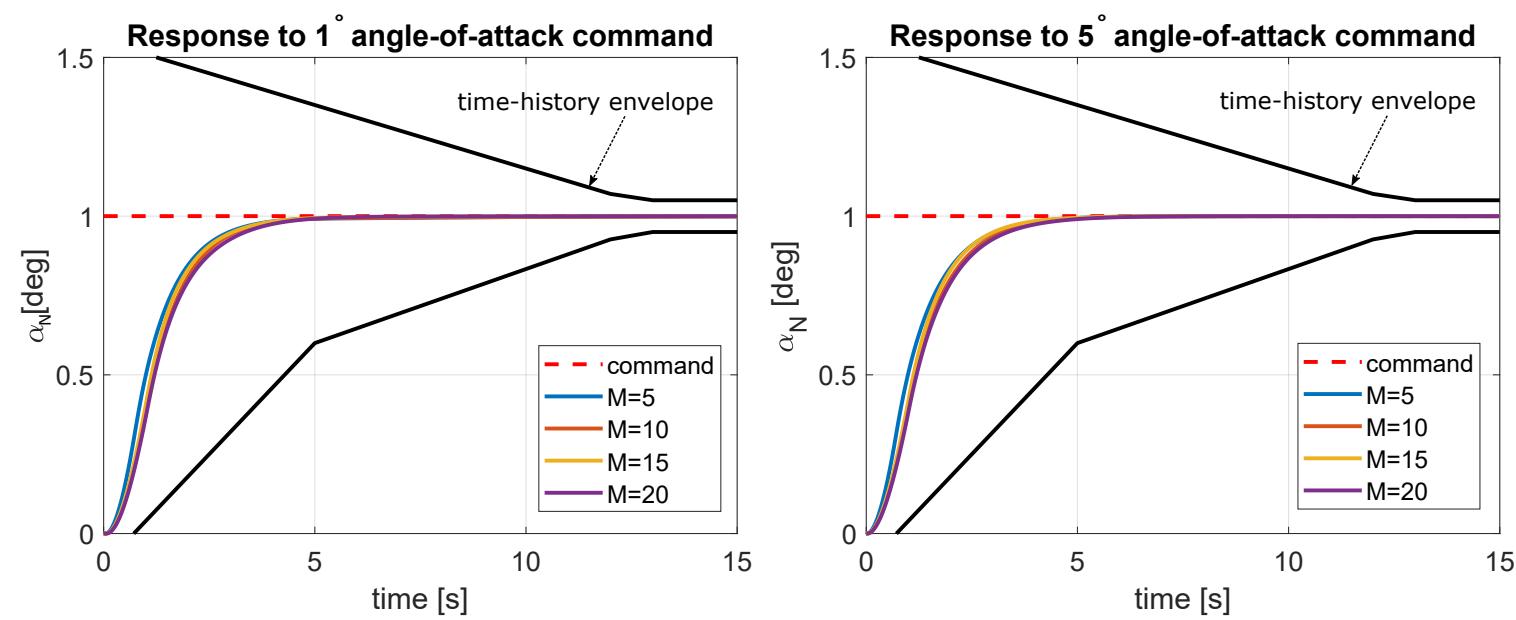

Figure 7.4: Characteristic normalised responses to an angle-of-attack command of the FSS-1 spaceplane along the ascent trajectory.

\subsection{Controllability}

The poor stability properties of the FSS-1, which were studied by the stability analyses in Chapter 5, require to design a robust control system for the FSS-1 reference vehicle to achieve and maintain the desired flight characteristics. In Chapter 6, the advanced adaptive controller was designed and, in Section 7.2, this baseline design was enhanced to be as insensitive to perturbations as possible. In this section, the capabilities of this control system are studied in relation with the controllability requirements defined by MIL-F-8785C [1980] and MIL-HDBK-1797 [1997].

The controllability specifications are given in two different forms for longitudinal and lateral motion. For the longitudinal motion, they are given as the maximum and minimum values of damping ratio, time rise, settling time, and maximum overshoot of the normalised response to an angle-of-attack step command. This means that the normalised transient response of a hypersonic vehicle to a angle-of-attack command shall be graphically bounded by a reference time-history envelope, which is shown in Figure 7.4 in black lines [DaimlerBenz Aerospace, 1998]. Figures 7.4 and 7.5 present the response of the system to two different angle-of-attack commands along the ascent and re-entry flight, respectively. The angles-of-attack deviation that are commanded are $1^{\circ}$ and $5^{\circ}$. The commands are imposed at flight conditions with $M=5,10,15,20$ along both the ascent and re-entry. The figures testify that the requirements are met in all the design points.

The requirements of controllability along the lateral motion are given in terms of time required to realise a $30^{\circ}$ bank-angle command, which are given in Table 7.3. Differently from the angle-of-attack commands that 

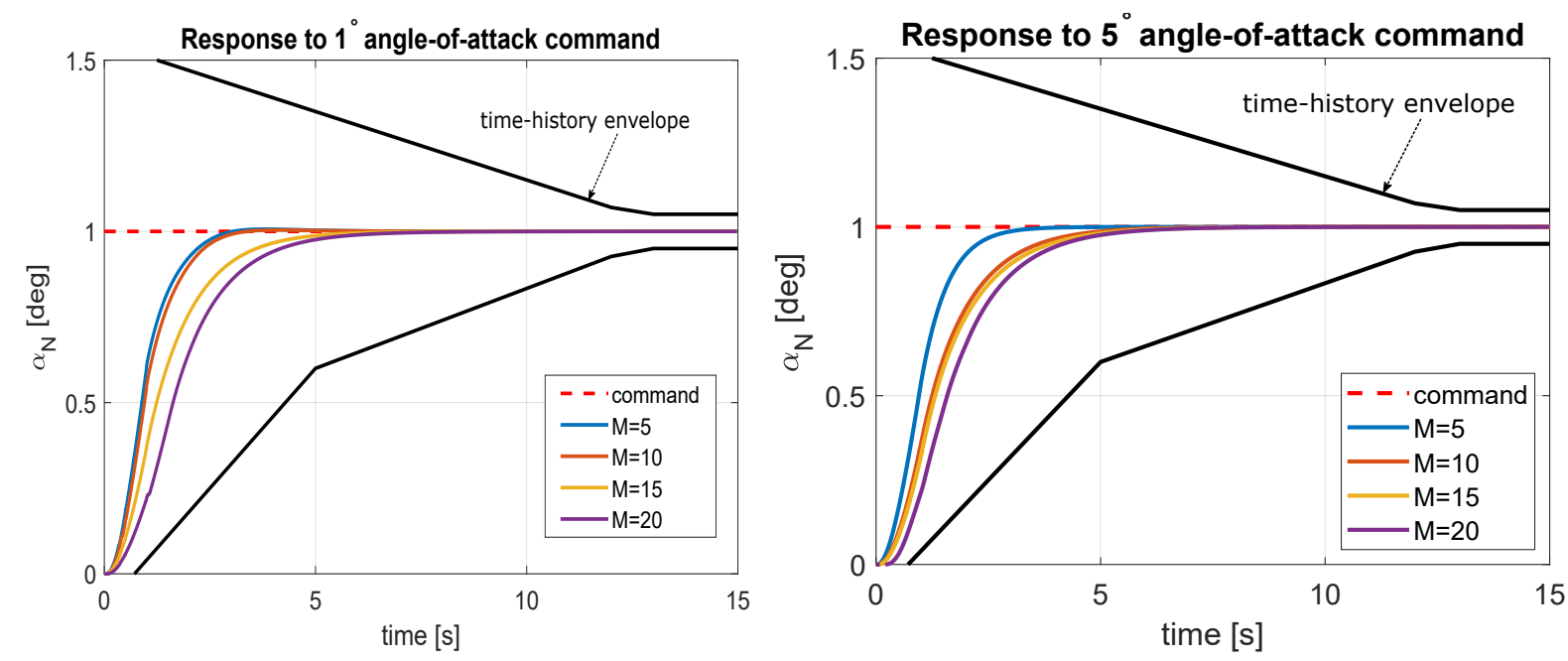

Figure 7.5: Characteristic normalised responses to an angle-of-attack command of the FSS-1 spaceplane along the re-entry trajectory.

Table 7.3: Roll control effectiveness criterion [Chalk, 1980].

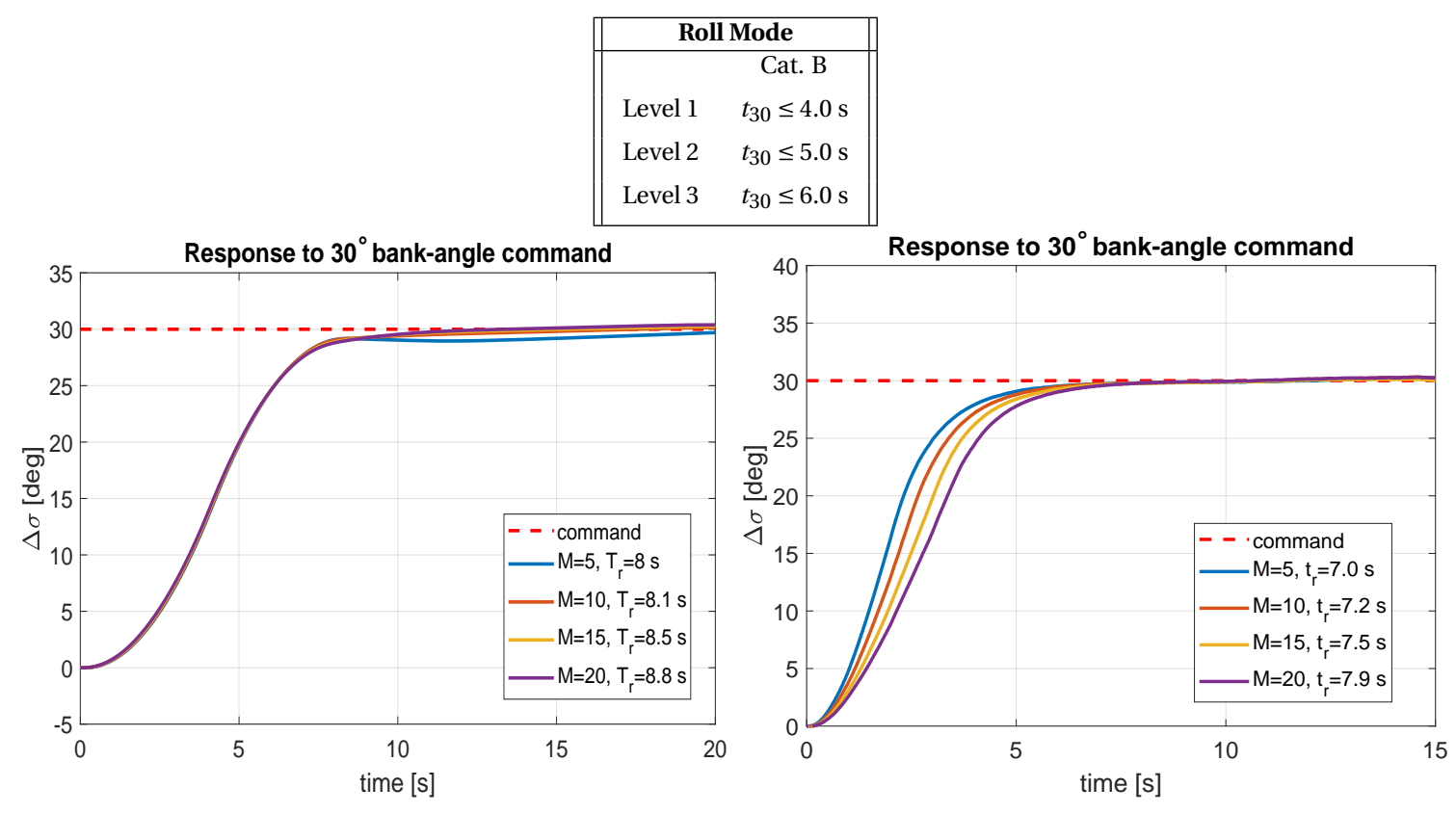

Figure 7.6: Characteristic responses to a $30^{\circ}$ bank-angle command along the FSS- 1 ascent (left) and re-entry (right) trajectory.

are step commands, the latter is a ramp because, when large variations are commanded, the impossibility of the system to instantaneously realise a command should be taken into account. The results are presented graphically in Figure 7.6, where the bank-angle variation $\Delta \sigma$ is plotted as function of time. In the plot legend, the rise time is specified.

Comparing these results with the MIL-Specs, it can be concluded that the system does not meet the MIL requirements along both the ascent and re-entry flight. It was proved that increasing the maximum allowable TVC deflections to $\pm 8^{\circ}$ improves the performance of the controller along the ascent; in particular it is possible to obtain $t_{r}<6$ s, i.e., Level 3. Obviously, the possibility of modifying the maximum TVC deflections needs to be verified, since it is linked to the structural limits of the vehicle. This is interesting to check and inspect, so it is left to future works. However, Chalk [1980] derives the roll control effectiveness limits as preliminary values to adapt the MIL-Specs to supersonic vehicles. Indeed, the military limits are considered too strict for high-speed vehicles. Thus, it is likely that for hypersonic spaceplanes even more relaxed limits of $t_{30}$ need to be specified. It is recommended that, during the future works, a similar analysis is conducted using another spaceplane so 

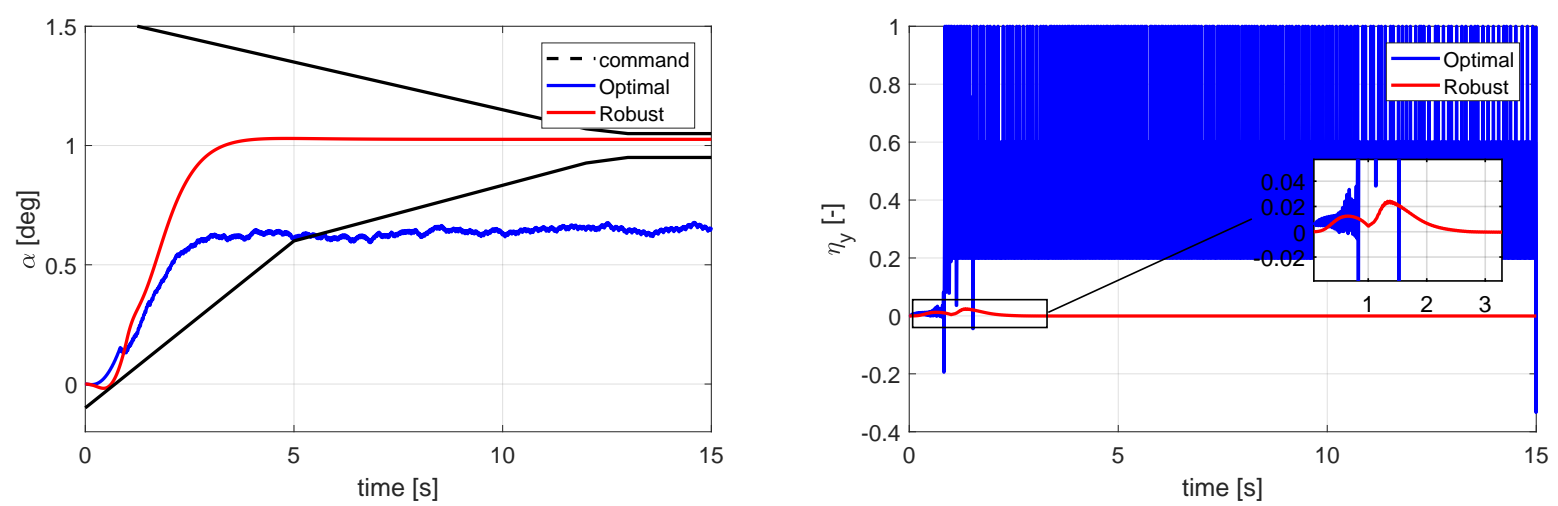

Figure 7.7: Influence of an error of $\pm 20 \%$ on the aerodynamic coefficients on the response to a $\alpha$ step command.

that the correctness of these limits for hypersonic speed can be ascertained. Overall, it can be concluded that the controller is able to realise the commands so that the desired flight conditions can be reached and the objectives of the mission can be achieved successfully.

Investigating the figures in details, some important features of the controller and the system itself can be recognised. Overall, it can be seen that, as expected, the time required to perform a command increases with increasing variation of $\alpha$ commanded. An interesting difference between the responses along the ascent flight and those along the re-entry flight is that the responses at different Mach number are almost the same during the ascent, while the higher the Mach number the slower the response during the re-entry. This is essentially due to the different actuators used in these two portions of flight. In fact, along the hypersonic re-entry trajectory, the control surfaces and the RCS thrusters are used to control the vehicle's motion. The control surfaces influence the motion by changing the aerodynamic moments, which are strongly dependent on the dynamic pressure. So, since the dynamic pressure decreases with increasing Mach number during the re-entry, the effectiveness of the aerodynamic surfaces decreases, resulting in a slower response.

Differently, along the ascent trajectory the control commands are executed by using TVC, which maintains an high effectiveness along the whole trajectory. The reason is that the thrust magnitude is quite large during the whole ascent flight, thus few degrees of thrust inclination are sufficient to realise the desired command. However, there could be some differences in response depending on Mach number and flight conditions due to the inertia of the propulsion system. The study of the influence of the propulsion system dynamics on the response to longitudinal and lateral commands is left to further works.

Finally, it seems interesting to consider the effect of an error of $\pm 20 \%$ of the aerodynamic coefficients on the response to a step command. At the same time, it is desired to compare the performance of the robust and optimal control-system designs in these conditions. In Figure 7.7 the results are shown in case of an angleof-attack step command. It can be seen that the optimal design is not able to handle the uncertainty of the aerodynamic data. Indeed, the final commanded angle of attack is not reached, since it realizes only the $60 \%$ of the command. Since it is not able to stabilise the system to the new commanded equilibrium condition, the control variable $\eta_{y}$ saturates after $1 \mathrm{~s}$ and starts oscillating rapidly. Differently, the robust design can realise the command requiring quite low control effort. Moreover, it can be highlighted that, even in presence of the aerodynamic uncertainty, the response remains within the time-history envelope, meeting the controllability requirement.

Also a comparison of the LQR and SAC performance is performed. The LQR controller is adequate to realise the control commands in case of nominal conditions. However, when uncertainties or disturbances are introduced in the system, it starts drifting away, requiring large deflections of the control surfaces. These large deflections can lead to diverging oscillations and a complete loss of control. This does not happen when the SAC controller is used, thanks to its adaptive gains. It can be concluded that, not only the SAC control system is able to stabilise the vehicle, but also the robust design is able to minimise the effect of uncertainties in the input parameters on the overall mission performance.

\subsection{Response to Atmospheric Disturbances}

The levels of flying qualities in open-loop and closed-loop studied until now are defined by MIL-F-8785C [1980] and MIL-HDBK-1797 [1997] to account for the possibility that the vehicle is required to operate under 

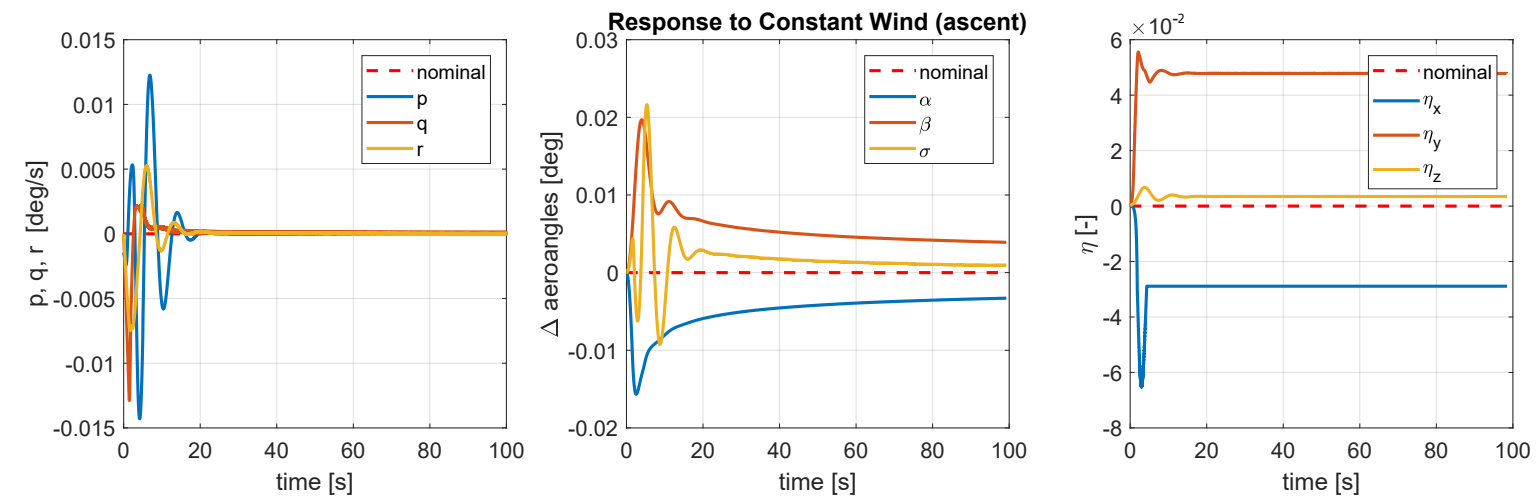

Figure 7.8: System response to steady state wind $\left(V_{W}=25 \mathrm{~m} / \mathrm{s}\right)$ at $\mathrm{M}=5$ along the ascent trajectory.


Figure 7.9: System response to steady state wind $\left(V_{W}=24.8 \mathrm{~m} / \mathrm{s}\right)$ at $\mathrm{M}=5$ along the re-entry trajectory.

abnormal conditions. Abnormalities that may occur can be atmospheric disturbances. In this case, a degradation of the flying qualities and controllability characteristics may be allowed. In this section the behaviour of the system in case of atmospheric disturbances is investigated.

The atmospheric disturbances that are studied are the constant wind, wind gusts, and turbulence. As presented in Chapter 3, the horizontal wind model HWM07 is used to model steady-state wind, while the wind gusts and turbulence are modelled using the 1-cosine formula and the Dryden spectral model, respectively. These disturbances shall be applied to the vehicle EoM through the aerodynamic terms, as shown in Section 4.6.3. As a consequence, it is underlined that the state variables that the controller will command are the aerodynamic angles with respect to the airspeed. However, since it is the task of the guidance system to provide the commands necessary to fly the desired trajectory, it is recommended for future works to further investigate whether it is preferable, from a guidance point of view, to control the aerodynamic angles with respect to airspeed or groundspeed. In the following sections the responses to steady-state wind, wind gusts, and turbulence are analysed.

\subsubsection{Response to Steady-State Wind}

When steady-state wind is applied along the hypersonic trajectory of the FSS-1 spaceplane, it is required that it withstands the disturbance using no more than $75 \%$ of the available control authority. This means that if the available authority is identified by $\eta_{x, y, z}=1$, the required control power shall be $\eta_{x, y, z_{\max }} \leq 0.75$.

The wind magnitude and direction angles are not chosen arbitrarily in the analysis, but the HMW07 is used to determine them on the basis of the position of the vehicle and time epoch expressed in day and seconds. The position of the vehicle derives from the nominal conditions of the design points along the FSS-1 hypersonic trajectory, and the time epoch chosen is an epoch in 2015, when the operational capabilities of the FESTIP concept was supposed to start. In the following the results are presented for the design point at $M=5$ of both the ascent and re-entry trajectory in Figure 7.8 and Figure 7.9, respectively, since these are the design points where the wind with larger amplitude is experienced. In these points, $V_{W}=25 \mathrm{~m} / \mathrm{s}$ along the ascent and $V_{W}=24.8 \mathrm{~m} / \mathrm{s}$ during the re-entry, which can be classified as storm (i.e., level 10 of the Beaufort scale [Singleton, 2008]). It can be noticed that in both flight conditions the system is able to converge to the nominal conditions, 
Table 7.4: Requirements on the response to constant wind and characteristics of the response of the FSS-1 system.

\begin{tabular}{|c|c|c|c|c|c|}
\hline \multicolumn{6}{|c|}{ Response to Constant Wind } \\
\hline \multirow{4}{*}{$\begin{array}{c}\eta_{\text {max }_{\text {ascent }}} \\
\eta_{\text {max }_{\text {re-entry }}}\end{array}$} & \multirow[t]{2}{*}{ Requirement } & \multicolumn{4}{|c|}{ FSS-1 Characteristics } \\
\hline & & $M=5$ & $M=10$ & $M=15$ & $M=20$ \\
\hline & 0.75 & 0.06 & 0.05 & 0.02 & 0.01 \\
\hline & 0.75 & 0.12 & 0.10 & 0.10 & 0.05 \\
\hline & \multicolumn{5}{|c|}{$\begin{array}{l}\text { Requirement not met } \\
\text { Requirement met }\end{array}$} \\
\hline
\end{tabular}

using low control power. Also, the moment fractions converge to a steady condition, which is maintained until the wind acts on the vehicle. In Figure 7.8 the rotational motion of FSS-1 under constant wind is simulated for $100 \mathrm{~s}$ to clearly show that both the state and control variables reach a stable condition, which account for the presence of wind.

Overall, some differences between the ascent and re-entry can be highlighted. The state deviations from the nominal conditions are lower along the ascent trajectory by a factor of about 100 . Also, the control required to stabilise the vehicle is lower in case of the ascent flight. Indeed, although the two points of the trajectory considered have the same Mach number, they are characterised by a completely different aerodynamic configuration. Given the strong non-linearity of the aerodynamic properties, the effects that the atmospheric disturbances can have on the vehicle motion are strongly dependent on the flight conditions, especially on $\alpha$ in this particular case. It would be interesting to examine in more details the relation between the aerodynamic configuration and the response to atmospheric disturbances, thus it is left to further research. However, during the ascent flight the convergence to the new stable condition seems slower. This can be addressed to the fact that the controller is more sensitive and responsive in case of larger deviations from the nominal state.

It was ascertained during the analysis that the stability condition can be reached by the system in all the points of the trajectory studied. It is remarkable that the higher the Mach number the lower the effect of wind on state variables and the lower is the control effort required to reach a stable condition. Moreover, once the stable condition is reached, it is possible that the aerodynamic angles have a steady-state error with respect to the nominal values. This is dependent on the accuracy requirement of the controller, which are set from the designer. For control performance reasons it is suitable to accept $5 \%$ error.

To identify if the MIL-Specs are met also in the other flight conditions, Table 7.4 gives the maximum moment fraction required to counteract the effect of steady-state wind and achieve a new stable condition. Here, the results for $M=5,10,15,20$ along the ascent and re-entry trajectory are presented. The response of the system to steady-state wind meets the MIL requirement at any flight condition. Furthermore, it is noticed that the actual maximum control power necessary to withstand to the constant wind is much lower than the MIL limits defined for subsonic vehicles. This can be linked to the much higher kinetic energy that characterises a hypersonic vehicle. As a consequence, we can deduce that the system can potentially withstand even more severe wind conditions. It can be concluded that the SAC controller is able to promptly stabilise the vehicle in case of a steady-state wind.

\subsubsection{Response to Wind Gusts}

The horizontal and vertical wind gusts are modelled using the 1-cosine formula. It is required that the vehicle shall withstand wind gusts with max amplitude of $12 \mathrm{~m} / \mathrm{s}$. To identify if the system met this requirement, a wind gust is applied at $t=5 \mathrm{~s}$. The cases of horizontal only and vertical only are studied. It is chosen to report here only the final case presenting a combination of horizontal and vertical gusts, so that both the effects can be studied in a more compact way. The wind velocity components are chosen so that it has a total magnitude of $12 \mathrm{~m} / \mathrm{s}$ and the horizontal and vertical components have the same magnitude, i.e., $V_{w, g}=(6,6,8) \mathrm{m} / \mathrm{s}$ along the $x$-, $y$ - and $z$ - axes.

Similarly to what was found for the case of steady-state wind, the system is able to restore the equilibrium. The speed of convergence of the response to the new stable conditions varies depending on the flight conditions. For instance, Figure 7.11 shows that when the system is subjected to a wind gust along its re-entry trajectory at $M=15$ the time required to reach stability is about $25 \mathrm{~s}$. However, at $M=20$ it was seen that this time increases to $50 \mathrm{~s}$, but here the state deviations induced by the wind gusts are smaller. Along the ascent 

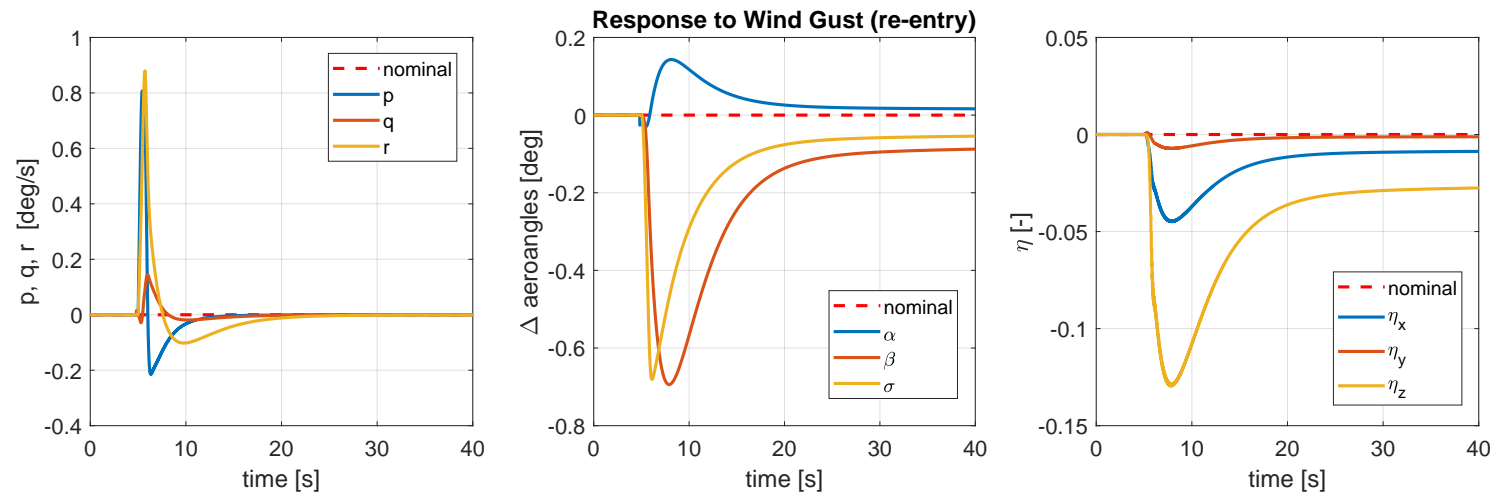

Figure 7.10: System response to wind gust of magnitude $12 \mathrm{~m} / \mathrm{s}$ at $M=15$ along the re-entry trajectory.


Figure 7.11: System response to wind gust of magnitude $12 \mathrm{~m} / \mathrm{s}$ at $M=15$ along the ascent trajectory.

trajectory, the wind gust induces smaller state deviation, but a larger time is required to stabilise the system. Figure 7.10 present the response to wind gusts at flight condition of $M=15$ along ascent, where the stability is obtained at about $t=60 \mathrm{~s}$. This value is doubled if we apply the same wind gust at $M=20$. In addition, we can noticed that during ascent the response of the lateral-directional variables oscillates converging to the new equilibrium value. This means that a similar perturbation triggers an oscillatory lateral mode of motion of the reference vehicle that ends when the new stable condition is reached. Differently, along the re-entry the system behaviour is a pure convergence to the new stable condition. Finally, also in case of response to wind gusts, the state variables can present a small steady-state error with respect to the nominal condition. However, as long as it is lower than the controller accuracy requirement (5\% error on the nominal value), it considered satisfactory for the analysis.

\subsubsection{Response to Turbulence}

Turbulence is modelled using the Dryden spectral model, which is detailed in Section 3.3.3 on the basis of what is defined in MIL-HDBK-1797 [1997]. The response of the system to turbulence is presented in Figure 7.12 for re-entry flight at $M=10$. Indeed, although the response changes depending on the point along the trajectory considered, the remarkable conclusions that can be drawn are the same.

In the presence of turbulence, the controller appears to be continuously excited due to the noisy trend that characterises the turbulence. The controller responds to minimise its effect and restore the nominal conditions. The differences between the ascent and re-entry flight that are identified in this case are similar to those encountered when studying steady-state wind and wind gusts. Indeed, also in this case the state deviations are slightly smaller along the ascent. The MIL specifications require that the vehicle shall withstand turbulence, modelled with Dryden spectral model, without reaching the control authority limits. Table 7.5 illustrates that the requirement is met both along ascent and re-entry flight. Also in this case, the low control effort required should be linked to the high kinetic energy at hypersonic speed, which makes spaceplane less sensitive to atmospheric disturbances.

An additional analysis is carried out to investigate the potentiality of our controller. Indeed, it can happen that the control system gets saturated when continuously excited. To evaluate if this happen also to our system, 

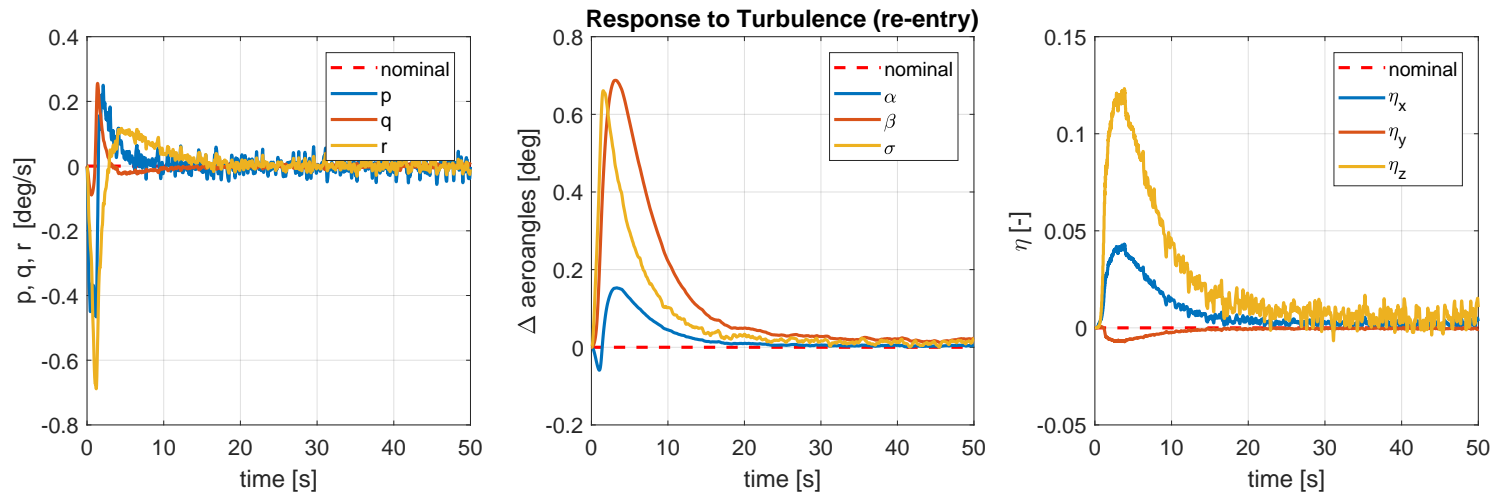

Figure 7.12: System response to perturbation at $M=10$ along the re-entry trajectory.

Table 7.5: Response to turbulence requirements and characteristics of the response of the FSS-1 system.

\begin{tabular}{|c|c|c|c|c|c|}
\hline \multicolumn{6}{|c|}{ Response to Turbulence } \\
\hline \multirow{4}{*}{$\begin{array}{c}\eta_{\max _{\text {ascent }}} \\
\eta_{\text {max }_{\text {re-entry }}}\end{array}$} & \multirow[t]{2}{*}{ Requirement } & \multicolumn{4}{|c|}{ FSS-1 Characteristics } \\
\hline & & $M=5$ & $M=10$ & $M=15$ & $M=20$ \\
\hline & 1 & 0.1 & 0.05 & 0.04 & 0.01 \\
\hline & 1 & 0.15 & 0.13 & 0.1 & 0.07 \\
\hline \multicolumn{6}{|c|}{ Legend } \\
\hline & \multirow{2}{*}{\multicolumn{5}{|c|}{ Requirement not met }} \\
\hline & & & \multicolumn{3}{|c|}{ Requirement met } \\
\hline
\end{tabular}

longer simulations of the response to turbulence are studied. It was pleasing to observe that, although the oscillatory behaviour remains as long as the turbulence acts on the system, this behaviour is maintained and the control gains do not saturate. Thus, it can be concluded that the controller makes the vehicle stable with respect to atmospheric disturbances of any kind. 


\section{8}

\section{Conclusions \& Recommendations}

The main research question of this thesis project has been be formulated as follows:

Which are the flying qualities of hypersonic spaceplanes, how do the environmental disturbances and design uncertainties influence them, and how can a control system compensate for any deficiency of the system and contribute to the mission success?

The main research question has been translated into six research opportunities that can be expressed by the following research sub-questions:

1. Which are the flying qualities of hypersonic spaceplanes?

2. How do the flying qualities of hypersonic spaceplanes change with respect to the flying qualities of subsonic vehicles embodied in MIL-F-8785C [1980] and MIL-HDBK-1797 [1997]?

3. What are the controllability characteristics of hypersonic spaceplanes?

4. What is the influence of environmental disturbances, such as steady-state wind, turbulence and wind gusts, on the system performance?

5. If environmental disturbances introduce some deficiency in the system, how can the control system compensate for it?

6. How sensitive is the control system to uncertainties in input parameters and what are their effects on the overall mission performance?

This chapter concludes this thesis report with the aim to answer the research questions. To this end, the research outcomes are summarised with particular focus on the contribution to the study of the flying qualities and controllability characteristics of hypersonic spaceplanes. This is treated in Section 8.1. Finally, proposals for future possible improvements and developments of this research topic are recommended in Section 8.2.

\subsection{Conclusions}

The main goal of the thesis project is derived from the need to study the flying qualities and controllability characteristics of hypersonic spaceplanes. Indeed, even though the flying qualities requirements for subsonic aircraft are completely determined by MIL-F-8785C [1980] and MIL-HDBK-1797 [1997], they constitutes an open research field for hypersonic vehicles that needs to be investigated. To this end, a simulator describing the vehicle's motion under the influences of forces and moments needed to be developed and a robust controller, which is able to compensate for any deficiency of the system, had to be designed. In the following the subquestions will be answered first, so that from there the main research questions can be addressed in a complete, concise and accurate manner.

The first and second sub-questions can be answered in one go, since they represent two sides of the same coin. For the sake of convenience they are repeated here:

1. Which are the flying qualities of hypersonic spaceplanes?

2. How do the flying qualities of hypersonic spaceplanes change with respect to the flying qualities of subsonic vehicles embodied in MIL-F-8785C [1980] and MIL-HDBK-1797 [1997]? 
Once the flying-quality requirements for the category of aircraft that has similar characteristics to hypersonic spaceplanes are identified, the stability of the latter can be analysed. The stability analysis was subdivided into three main parts: the study of the spaceplane's eigenmotion along its hypersonic ascent and re-entry trajectory, the examination of the trim capabilities and, finally, the investigation of the flying qualities. It was revealed that the spaceplane presents almost the same modes of motion of conventional aircraft, but fundamental differences exist. For instance, apart from presenting an additional mode of motion, i.e., the height mode, the short-period has a much larger period (about 30 times), a degeneration of complex mode into two separated modes can generally happen, and conventionally periodic mode can be aperiodic, such as the lateral oscillation. From the eigenmotion analysis, the vehicle resulted unstable overall. Moreover, despite trim helps to reduce the instabilities of the system, especially for the longitudinal motion, it does not suffice to stabilise the spaceplane. Finally, the identified stability characteristics were compared with the flying-qualities requirements specified by MIL-F-8785C [1980] and MIL-HDBK-1797 [1997]. It turns out that only the phugoid requirements are met, achieving Level 1 in the design points along the ascent and Level 3 along the re-entry. This is linked to the difference angle of attacks that characterise these two portions of flight. The MIL requirements about the short period and lateral oscillations are not met, with very large violations that can go from $10 \%$ to $50 \%$. Although, the differences between the speed regimes can require a modification of the requirements that are specific for subsonic vehicle, this thesis application does not manifest this need. In fact, the outcome of the flying-quality analysis for the reference hypersonic vehicle in open-loop confirms the conclusions drawn from the eigenmotion and trimmability analyses, i.e., the FSS-1 reference vehicle is dynamically unstable and needs to be controlled to perform a stable flight and achieve the mission objectives.

The third sub-question is answered in the following:

\section{What are the controllability characteristics of hypersonic spaceplanes?}

Being the open-loop system unstable along its hypersonic trajectory, there is the need to integrate it with an efficient control system that is able to stabilise it. The control system has been designed starting from the simple and commonly used LQR controller, which functions as benchmark for the verification of the more advanced controller. Afterwards, the control performance is enhanced by designing a more advanced control system, i.e., the adaptive controller. This is chosen to conduct the analysis mainly because it improve the system response to control commands, being characterised by adaptive gains that makes it less sensible to any kind of disturbances. The controllability requirements for the longitudinal motion are defined in form of time-history envelope, where the normalised response to an angle-of-attack command shall be bounded. For the lateral motion, they are defined in terms of maximum time required to realise a $30^{\circ}$ bank-angle command. It resulted that Level 1 was obtained for controllability characteristics of the longitudinal motion for both ascent and reentry, while for the lateral motion the requirement is not met. However, the response to bank-angle commands are quite satisfactory in terms of integrated control error and effort, thus it is possible that more relaxed limits should be specified for hypersonic spaceplanes due to the slower dynamics with respect to subsonic vehicles.

The two following sub-questions are intimately related. Consequently, a unified answer can be beneficial. They demands:

4. What is the influence of environmental disturbances, such as steady-state wind, wind gusts and turbulence, on the system performance?

5. If environmental disturbances introduce some deficiency in the system, how can the control system compensate for it?

As expected, the atmospheric disturbances may degrade the stability and controllability properties of the vehicle. However, the flying quality and controllability requirements are defined in the MIL-Specs so that the vehicle is able to operated not only under nominal conditions, but also in case of abnormalities. The most common abnormal conditions to which the vehicle can be subjected are the atmospheric disturbances, such as steadystate wind, wind gusts and turbulence. As indicated in MIL-F-8785C [1980], the wind gusts and turbulence shall be modelled using the 1-cosine formula and the Dryden spectral model, respectively, while it is chosen to model the steady-state wind with the horizontal wind model, HWM07. The control system was essential to avoid a degradation of the flying qualities and the controllability characteristics in case of atmospheric disturbances. Indeed, if any kind of the atmospheric disturbances cited before acts on the system, the control system operates so that the the system converges to the nominal conditions. This is achieved using a control power that is well within the military specifications. Once the vehicle is stabilised, the control variables converge to a steady condition that is maintained as long as the perturbations act on the vehicle. So, although the time to reach the new stable condition in response to atmospheric disturbance can vary depending on the flight conditions, the control system is able to make the vehicle stable with respect to atmospheric disturbances without 
reaching a saturation of the control gains.

Finally, the last question needs to be addressed:

6. How sensitive is the control system to uncertainties in input parameters and what are their effects on the overall mission performance?

Uncertainties in input parameters have to be taken into account when designing a new complex system since, being unforeseeable and non-quantifiable, can alter the performance of the design. In our application, the initial conditions, mass and inertia properties, atmospheric properties, and aerodynamic coefficients can be affected by uncertainty. To deal with it, we desire the control system to be as insensitive as possible to uncertainties without eliminating the source of the uncertainties. A control system with this property is defined as a robust control system. To this end, a Robust Design technique is applied. It consists of a double-loop sensitivity analysis, where the controller-design parameters are varied in the outer loop and the noise factors (or, in other words, the parameters affected by uncertainty) are varied in the inner loop. Here, the signal-to-noise ratio is used to quantify the influence of the uncertainty on the performance. At the end of the analysis, the robust design of the control system was identified. When this design is used, the effects of the uncertainties of the input parameters on the mission performance is minimised and it is guaranteed that the vehicle can realise the mission objectives, while performing a stable flight. The performance of the robust adaptive controller was compared with the one of the baseline controller. It results that the baseline controller is not able to deal with the uncertainties: the commanded aerodynamic angles are not reached and the control variables get saturated and are affected by very rapid oscillations. Differently, the robust controller can realise the attitude commands, minimising the effect of the uncertainties on the overall performance.

In conclusion, the main research question can be answered:

Which are the flying qualities of hypersonic spaceplanes, how do the environmental disturbances and design uncertainties influence them, and how can a control system compensate for any deficiency of the system and contribute to the mission success?

The present thesis research has demonstrated that a spaceplane along its hypersonic ascent and re-entry trajectory is characterised by poor flying qualities and stability characteristics, which would be even more degraded in case of perturbations (e.g., environmental disturbances and design uncertainties). Indeed, the open-loop system does not meet the MIL-requirements. However, when a robust and advanced control system is integrated into the system, the spaceplane becomes stable in all the flight conditions analysed. A robust design of the controller is developed, by means of a double-loop sensitivity analysis, so that the control system results as insensitive to uncertainties as possible. In this way, the presence of uncertainties can be promptly counteracted and their influence on the performance is minimised. The response to control commands of the longitudinal motion is generally rated with Level 1 of the MIL-Specs. The response to lateral-directional commands does not meet the actual requirements. However, it is likely that these are too strict for hypersonic vehicles and more relaxed limits should be allowed. Also, the system can withstand to atmospheric disturbances. Indeed, the controller is able to recover the nominal conditions, stabilising the control variables to a new steady situation and requiring low control effort. This is linked to the high kinetic energy at hypersonic speed that reduces the deviations induced by the perturbations. It can be concluded that a robust control system is essential for a hypersonic spaceplane to perform a stable flight and achieve the mission objectives.

\subsection{Recommendations}

An analysis as the one conducted in this thesis research can be extended with more complex models that can provide a better approximation of the system and, consequently, a broader picture of its capability and performance. In the following the recommendations for further research are offered.

Rec-1 In this thesis research, the flying qualities and controllability characteristics of hypersonic spaceplanes were studied at some key design points along the ascent and re-entry trajectory. Although this was appropriate to define the representative behaviour of hypersonic spaceplanes, extending the analysis along the whole trajectory would be beneficial to the analysis. In this way, the evolution of the eigenmotion over time could be deeply studied and the performance of the control system can be investigated also at other flight conditions. For a full-flight simulation, a guidance system to generate the steering commands shall be introduced in the simulator.

Rec-2 Similarly, it would be interesting to design the navigation system and integrate it with the existing simulator. So, the effects of the measurement inaccuracies and model mispredictions on the performance 
can be evaluated and, if necessary, any deficiency can be corrected.

Rec-3 Related to the Rec-1, during this thesis work the flying qualities and controllability characteristics of spaceplanes were studied along the hypersonic ascent and re-entry trajectory. However, there are some other manoeuvres in hypersonic missions where it is essential to study the stability and controllability properties. For instance, as emphasised by the studies by Jacob et al. [2005] and Chalk [1980], a typical flight manoeuvre, which can be used for solving flying-quality issues in supersonic and hypersonic regimes, is a steady-level turn at constant bank angle of $30^{\circ}$ during the cruise flight. MIL-Specs define the flying qualities and controllability characteristics that can be used to examine the manoeuvrability capacity of a vehicle. Also in this case, a guidance control needs to be designed.

Rec-4 For controllability and stability studies, it could be interesting to see how the control system behaves in case of off-nominal situation of the mass distribution, i.e., when there is an offset in the centre-of-mass position. For instance, this can happen when for some reason only the left/right tank is used or one of the rockets is not working. These cases will be taken into account for further development of this research topic.

Rec-5 Generally, actuators are modelled as ideal elements, so they are assumed to be perfect and to provide immediate actuation. This approximation was used in this project. This is a valid assumption in the majority of the cases. However, to design the control system performance to a higher precision, the actuator dynamics need to be included. For future possible improvements of this thesis work, it is proposed to consider the impact of the actuators dynamics on the flying qualities and controllability analysis. 


\section{Bibliography}

Beal, T. R. [1993]. "Digital Simulation of Atmospheric Turbulence for Dryden and von Karman Models”, Journal of Guidance, Control, and Dynamics, volume 16, no. 1, pp. 132-138.

Bentley, M. A. [2009]. Spaceplanes: From Airport to Spaceport, Springer.

Brinkman, D. [2017]. “Knowledge-based Control Systems for Re-entry Vehicles”, Msc thesis, Delft University of Technology, Faculty of Aerospace Engineering, Repository - uuid:b77b4495-d42b-46be-9a9c-306f49bfbe32.

Camacho, E. F. and Alba, C. B. [2007]. Model Predictive Control, Springer.

Carmona Castillo, J. and Sanchez Gonzales Cantalejo, P. [1993]. "GNC for advanced launch vehicle - Guidance and navigation conceptual analysis”, Technical Note 300, ESA contract no. 9734/NL/JG, GMV S.A., 2055/93 V2/93.

Chalk, C. R. [1980]. Flying Qualities Design Criteria Applicable to Supersonic Cruise Aircraft, NASA. Langley Res. Center Supersonic Cruise Res. 1979, Pt. 1; p 251-267, doi:19810009468.

Cornelisse, J. W., Schoyer, H. F. R., and Wakker, K. F. [1979]. Rocket propulsion and spaceflight dynamics, Pitman, London.

Cotting, M. C. [2010]. Evolution of Flying Qualities Analysis: Problems for a New Generation of Aircraft, PhD Thesis, Aerospace Engineering, Faculty of the Virginia Polytechnic Institute and State University.

Cox, T. H. and Jackson, D. [1997]. "Supersonic Flying Qualities Experience Using SR-71”, Atmospheric Right Mechanics Conference, New Orleans, Louisiana, August 11-13.

Cox, T. H., Sachs, G., Knoll, A., and Stich, R. [1995]. “A Flying Qualities Study of Longitudinal Long-Term Dynamics of Hypersonic Planes”, AIAA, Planes and Hypersonics Technologies Conference, Chattanooga, Tennessee, April 3-7, AIAA PAPER 95-6150.

Daimler-Benz Aerospace [1998]. FESTIP System Concept Description, Daimler-Benz Aerospace, ESA.

Drob, D. P., Emmert, J. T., Crowley, G., Picone, J. M., Shepherd, G. G., Skinner, W., Hays, P., Niciejewski, R. J., Larsen, M., She, C. Y., Meriwether, J. W., Hernandez, G., Jarvis, M. J., Sipler, D. P., Tepley, C. A., O’Brien, M. S., Bowman, J. R., Wu, Q., Murayama, Y., Kawamura, S., Reid, I. M., and Vincent, R. A. [2008]. “An empirical model of the Earth's horizontal wind fields: HWM07”, Journal of Geophysical Research - Space Physics, Vol. 113, A12304, volume 113, no. A12, doi:10.1029/2008JA013668.

Duke, E. L., Antoniewicz, R. F., and Krambeer, K. D. [1988]. "Derivation and Definition of a Linear Aircraft Model", NASA Reference Publication 1207.

Durham, W. C. [1993]. “Constraint Control Allocation”, Journal of Guidance, Control, and Dynamics, volume 16, no. 4, pp. 717-725.

Etkin, B. and Reid, L. D. [1995]. Dynamics of Flight Stability and Control, 3rd edition, John Wiley \& Sons, Inc.

Gopal, M. [1989]. Modern control system theory, 2nd edition, Wiley Eastern Ltd., New Delhi.

Jacob, D., Sachs, G., and Wagner, S. [2005]. Basic Research and Technologies for Two-Stage-To-Orbit Vehicles, WILEY-VCH Verlag GmbH \& Co. KGaA, Weinheim.

Kaufman, H., BarKana, I., and Sobel, K. [1998]. Direct Adaptive Control Algorithms, 2nd edition, Springer-Verlag, New York.

Koelle, D. E. and Kuczera, H. [1989a]. “Saenger II, an advanced launcher system for Europe”, Acta Astronautica, volume 19, no. 1, pp. 63-72, doi:0094-5765/89. 
Koelle, D. E. and Kuczera, H. [1989b]. “Saenger II, an Advanced Launcher System for Europe”, Acta Astronautica Vol. 19, Nr. 1.

Kuipers, J. [2002]. Quaternions and Rotation Sequences, 5th edition, Princeton University Press.

Kuo, B. [1987]. Automatic control systems, Prentice Hall, Inc., Englewood Cliffs, New Jersey.

Lewis, F. L., Vrabie, D., and Syrmos, V. L. [2012]. Optimal Control, 3rd edition, John Wiley \& Sons, Inc.

MBB [1988]. “Study on Re-entry Guidance and Control -Final Report”, Space Communication and Propulsion System.

Mehta, U. B. and Bowles, J. V. [2001]. “A Two-Stage-to-Orbit Spaceplane Concept with Growth Potential”, AIAA2001-1795, International Space Planes and Hypersonic Systems and Technologies Conference, Kyoto, Japan, April 24-27.

MIL-F-8785C [1980]. Military Specification Flying Qualities of Piloted Airplanes, NASA.

MIL-HDBK-1797 [1997]. Flying Qualities of Piloted Airplanes, Handbook, Department of Defence, NASA.

Miller, C. [2011]. "Nonlinear Dynamic Inversion Baseline Control Law: Architecture and Performance Predictions", in AIAA Guidance, Navigation, and Control Conference, AIAA 2011-6467, Guidance, Navigation, and Control and Co-located Conferences, American Institute of Aeronautics and Astronautics, doi:10.2514/6. 2011-6467.

Mooij, E. [1994]. The Motion of a Vehicle in a Planetary Atmosphere.

Mooij, E. [1998a]. “Aerospace-plane Flight Dynamics, Analysis of Guidance and Control Concepts”, Phd thesis, Delft University of Technology.

Mooij, E. [1998b]. Linear quadratic regulator design for an unpowered winged re-entry vehicle, Delft University Press.

Mooij, E. [2002]. "Evolutionary Optimisation of a Model Reference Adaptive Control System", in AIAA Guidance, Navigation, and Control Conference and Exhibit, Guidance, Navigation, and Control and Co-located Conferences, American Institute of Aeronautics and Astronautics, doi:10.2514/6.2002-4922.

Mooij, E. [2004]. "Simple Adaptive Bank-Reversal Control of a Winged Re-entry Vehicle", in AIAA Guidance, Navigation, and Control Conference and Exhibit, Guidance, Navigation, and Control and Co-located Conferences, American Institute of Aeronautics and Astronautics, doi:10.2514/6.2004-4869.

Mooij, E. [2013]. “Characteristic Motion of Re-entry Vehicles”, in AIAAAtmospheric Flight Mechanics (AFM) Conference, Guidance, Navigation, and Control and Co-located Conferences, American Institute of Aeronautics and Astronautics, doi:10.2514/6.2013-4603.

Mooij, E. [2014]. "Passivity analysis for a winged re-entry vehicle", AIP Conference Proceedings, volume 1637, no. 1, pp. 1362-1376, doi:10.1063/1.4907302.

Mooij, E. [2015]. Re-entry Systems, Faculty of Aerospace Engineering, Delft University of Technology.

Mooij, E. [2017]. “Simple Adaptive Control System Design Trades”, AIAA Guidance, Navigation, and Control Conference, doi:10.2514/6.2017-1502.

Mooij, E. [2018]. “Simple Adaptive Re-entry Control with Actuator Assignment”, in 2018 ALAA Guidance, Navigation, and Control Conference, AIAA SciTech Forum, American Institute of Aeronautics and Astronautics, doi:10.2514/6.2018-1314.

Mooij, E. and Ellenbroek, M. [2011]. "Multi-Functional Guidance, Navigation and Control Simulation Environment - Rapid Prototyping of Space Simulations”, in D. M. Hoque (editor), Rapid Prototyping Technology Principles and Functional Requirements, InTech, pp. 315-338.

Mooij, E. and Wijnands, Q. [2002]. "Generic Attitude and Orbit Control Simulator development supporting the AOCS software life cycle”, in 7th International Workshop on Simulation for European Space Programmes, 
Noordwijk, The Netherlands.

Mooij, H. A. [1985]. Criteria For Low-Speed Longitudinal Handling Qualities of Transport Aircraft with ClosedLoop Flight Control Systems, Springer.

NASA [1977]. “Euler Angles, Quaternions, and Transformation Matrices”, NASA Mission Planning and Analysis Division, volume NASA-TM-74.

NASA [2000]. “Terrestrial Environment (Climatic) Criteria Handbook for Use in Aerospace Vehicle Development”, NASA-HDBK-1001.

NOAA, NASA, and USAF [1976]. “U.S. Standard Atmosphere”, NASA-TM-X-74335, NOAA-S/T-76-1562.

Ogata, K. [2010]. “Modern Control Engineering”, Prentice Hall.

Palais, B. and Palais, R. [2007]. “Euler's fixed point theorem: The axis of a rotation”, Journal of Fixed Point Theory and Applications, volume 2, pp. 215-220, doi:10.1007/s11784-007-0042-5.

Phadke, M. S. [1989]. Quality Engineering Using Robust Design, New Jersey.

Press, W., Teukolsky, S., Vetterling, W., and Flannery, B. [2007]. Numerical Recipies - The art of scientific computing, 3rd edition, Cambridge University Press.

Rijnsdorp, J. [2017]. "Performance of a Flush Airdata Sensor in a Particle Filter-Based Re-entry Navigation System", Msc thesis, Delft University of Technology, Faculty of Aerospace Engineering, Repository uuid:993ccea0-0839-4bf3-8662-76ebb37e6c61.

Roskam, J. [2001]. Airplane Flight Dynamics and Automatic Flight Controls, Part I, DARcorporation.

Sachs, G. [1993]. “Stability and Control Problems in Hypersonic Flight”, Space Course Munich.

Shaughnessy, J. D., Pickney, S. Z., McMinn, J. D., Cruz, C. I., and Kelley, M.-L. [1990]. "Hypersonic Vehicle Simulation Model: Winged-Cone Configuration.”, Technical report, NASA, National Aeronautics and Space Administration, NASA TM-102610, Hampton, Virginia.

Singleton, F. [2008]. “The Beaufort scale of winds - its relevance, and its use by sailors”, Weather, volume 63, no. 2, pp. 37-41, doi:10.1002/wea.153.

Turner, M. J. L. [2005]. Rocket and Spacecraft Propulsion, volume 53, Springer, doi:10.1017/CBO9781107415324. 004.

Wakker, K. F. [2015]. Fundamentals Of Astodynamics, Faculty of Aerospace Engineering, Delft University of Technology.

Wu, S.-F., Engelen, C., Babuska, R., Chu, Q.-P., and Mulder, J. [2000]. “Intelligent flight controller design with fuzzy logic for an atmospheric re-entry vehicle”, 38th Aerospace Sciences Meeting and Exhibit, pp. 1-12, doi: $10.2514 / 6.2000-174$.

Young, K. D., Utkin, V. I., and Ozguner, U. [1999]. “A control engineer's guide to sliding mode control”, IEEE Transactions on Control Systems Technology, volume 7, no. 3, pp. 328-342, doi:10.1109/87.761053. 


\section{State-Space Matrix Coefficients}

In the following the state-space form of the linearised EoM is presented. To linearise the non-linear EoM defined in Section 4.6, some assumptions need to be made:

- the oblateness and the rotation of the Earth has a second-order effect, thus we can assume that the Earth is spherical and non-rotating $\left(\omega_{c b}=0 \mathrm{rad} / \mathrm{s}\right)$;

- the vehicle can be considered to be rotational symmetric (in mass), i.e., presenting two plane of symmetry, and, consequently, all the products of inertia are zero, i.e., $I_{x y}=I_{y z}=I_{x z}=0$

- the vehicle's attitude does not depend on the asymmetrical translational motion, which means that the trajectory is parallel to the equator

With these assumptions, the non-linear equations of motion (Eqs. from (4.38) to (4.46) for the translational motion, and Eqs. from (4.53) to (4.58) for the rotational motion) become:

Equations of translational motion:

$$
\begin{gathered}
\dot{V}=\frac{F_{V}}{m} \\
V \dot{\gamma}=\frac{F_{\gamma}}{m}+\frac{V^{2}}{R} \cos \gamma \\
V \cos \gamma \dot{\chi}=\frac{F_{\chi}}{m}+\frac{V^{2}}{R} \cos ^{2} \gamma \tan \delta \sin \chi \\
\dot{R}=\dot{h}=V \sin \gamma \\
\dot{\tau}=\frac{V \sin \chi \cos \gamma}{R \cos \delta} \\
\dot{\delta}=\frac{V \cos \chi \cos \gamma}{R}
\end{gathered}
$$

Equations of rotational motion:

$$
\begin{gathered}
\dot{p}=\frac{M_{x}}{I_{x x}}+\frac{I_{y y}-I_{z z}}{I_{x x}} q r \\
\dot{q}=\frac{M_{y}}{I_{y y}}+\frac{I_{z z}-I_{x x}}{I_{y y}} p r \\
\dot{r}=\frac{M_{z}}{I_{z z}}+\frac{I_{x x}-I_{y y}}{I_{z z}} q p \\
\dot{\alpha}=q-(p \cos \alpha+r \sin \alpha) \tan \beta-\frac{L-m g \cos \gamma \cos \sigma}{m V \cos \beta} \\
\dot{\beta}=p \sin \alpha-r \cos \alpha-\frac{S-m g \cos \gamma \sin \sigma}{m V} \\
\dot{\sigma}=-\frac{p \cos \alpha+r \sin \alpha}{\cos \beta}-\frac{L-m g \cos \gamma \cos \sigma}{m V} \tan \beta+\frac{L \sin \sigma+S \cos \sigma}{m V} \tan \gamma
\end{gathered}
$$


with $g=\frac{\mu}{R^{2}}$, and $F_{V}, F_{\gamma}, F_{\chi}$ defined in Eqs. (4.41), (4.42), (4.43), respectively. Here, all the variables are calculated with respect to the groundspeed, however the subscript "g" is dropped for the sake of convenience. With the made assumptions the equations for $\chi, \tau$, and $\delta$ are not coupled with the other equations. Consequently, the linearised EoM in state-space form can be defined by 9 equations for $V, \gamma, R, p, q, r, \alpha, \beta, \sigma$.

It is convenient that the set of linearised equations of motion, for which the linearisation procedure is detailed by Mooij [2015], is written in state-space form. As specified in Section 4.7, the full set of EoM with translational and rotational equations for the open-loop system (i.e., no control) is necessary for the stability analyses. Also, the state-space model for the close-loop system is required for control-system design purposes. In this case, the EoM set can be reduced to consider the rotational motion only, by setting $\Delta \dot{V}=\Delta \dot{\gamma}=\Delta \dot{R}=\Delta V=\Delta \gamma=$ $\Delta R=0$. The linearised set of equations in state-space form can be written as:

$$
\dot{\mathbf{x}}=\mathbf{A x}+\mathbf{B u}
$$

where $\mathbf{x}$ is the state deviation vector with dimensions $n \times 1, \mathbf{u}$ is the deviation control vector with dimensions $m \times 1, \mathbf{A}$ is the state coefficient matrix with dimensions $n \times n$, and $\mathbf{B}$ is the control coefficient matrix with dimensions $n \times m$.

\section{A.1 Open-loop Model}

Only the homogeneous part of the state-space EoM is required for the study of the open-loop behaviour of a vehicle:

$$
\dot{\mathbf{x}}=\mathbf{A x}
$$

or fully written out:

$$
\left(\begin{array}{c}
\Delta \dot{V} \\
\Delta \dot{\gamma} \\
\Delta \dot{R} \\
\Delta \dot{p} \\
\Delta \dot{q} \\
\Delta \dot{r} \\
\Delta \dot{\alpha} \\
\Delta \dot{\beta} \\
\Delta \dot{\sigma}
\end{array}\right)=\left[\begin{array}{ccccccccc}
a_{V V} & a_{V \gamma} & a_{V R} & 0 & 0 & 0 & a_{V \alpha} & 0 & 0 \\
a_{\gamma V} & a_{\gamma \gamma} & a_{\gamma R} & 0 & 0 & 0 & a_{\gamma \alpha} & a_{\gamma \beta} & a_{\gamma \sigma} \\
a_{R V} & a_{R \gamma} & 0 & 0 & 0 & 0 & 0 & 0 & 0 \\
0 & 0 & 0 & 0 & 0 & 0 & 0 & a_{p \beta} & 0 \\
a_{q V} & 0 & 0 & 0 & 0 & 0 & a_{q \alpha} & 0 & 0 \\
0 & 0 & 0 & 0 & 0 & 0 & 0 & a_{r \beta} & 0 \\
a_{\alpha V} & a_{\alpha \gamma} & a_{\alpha R} & 0 & a_{\alpha q} & 0 & a_{\alpha \alpha} & 0 & a_{\alpha \sigma} \\
a_{\beta V} & a_{\beta \gamma} & a_{\beta R} & a_{\beta p} & 0 & a_{\beta r} & 0 & a_{\beta \beta} & a_{\beta \sigma} \\
a_{\sigma V} & a_{\sigma \gamma} & 0 & a_{\sigma p} & 0 & a_{\sigma r} & a_{\sigma \alpha} & a_{\sigma \beta} & a_{\sigma \sigma}
\end{array}\right]\left(\begin{array}{c}
\Delta V \\
\Delta \gamma \\
\Delta R \\
\Delta p \\
\Delta q \\
\Delta r \\
\Delta \alpha \\
\Delta \beta \\
\Delta \sigma
\end{array}\right)
$$

where elements $a_{i j}$ of matrix $\mathbf{A}$ are specified as follows [Mooij, 2015]:

$$
\begin{gathered}
a_{V V}=-\frac{1}{m V_{0}}\left(M_{0} \frac{\partial C_{D}}{\partial M} \bar{q}_{0} S_{r e f}+2 D_{0}\right) \\
a_{V \gamma}=-g_{0} \cos \gamma_{0} \\
a_{V R}=2 \frac{g_{0}}{R_{0}} \sin \gamma_{0} \\
a_{V \alpha}=\frac{T_{0}}{m} \sin \left(\epsilon_{0}-\alpha_{0}\right)-\frac{1}{m} \frac{\partial C_{D}}{\partial \alpha} \bar{q}_{0} S_{r e f} \\
a_{V p}=a_{V q}=a_{V r}=a_{V \beta}=a_{V \sigma}=0 \\
V_{0}\left(-\dot{\gamma}_{0}+\frac{2 V_{0}}{R_{0}} \cos \gamma_{0}\right)+\frac{\cos \sigma_{0}}{m V_{0}^{2}}\left(M_{0} \frac{\partial C_{L}}{\partial M} \bar{q}_{0} S_{r e f}+2 L_{0}\right) \\
a_{\gamma \gamma}=-\left(\frac{V_{0}}{R_{0}}-\frac{g_{0}}{V_{0}}\right) \sin \gamma_{0} \\
a_{\gamma R}=\left(\frac{2 g_{0}}{V_{0}}-\frac{V_{0}}{R_{0}}\right) \frac{\cos \gamma_{0}}{R_{0}} \\
a_{\gamma \alpha}=\frac{\cos \sigma_{0}}{m V_{0}} \frac{\partial C_{L}}{\partial \alpha} \bar{q}_{0} S_{r e f}+\frac{T_{0} \cos \sigma_{0}}{m V_{0}} \cos \left(\alpha_{0}+\epsilon_{0}\right)
\end{gathered}
$$




$$
\begin{aligned}
& a_{\gamma \beta}=-\frac{\sin \sigma_{0}}{m V_{0}} \frac{\partial C_{S}}{\partial \beta} \bar{q}_{0} S_{r e f}-\frac{T_{0} \sin \sigma_{0}}{m V_{0}} \cos \left(\alpha_{0}+\epsilon_{0}\right) \\
& a_{\gamma \sigma}=-\frac{L_{0}}{m V_{0}} \sin \sigma_{0}+\frac{T_{0}}{m V_{0}} \sin \sigma_{0} \sin \left(\alpha_{0}+\epsilon_{0}\right) \\
& a_{\gamma p}=a_{\gamma q}=a_{\gamma r}=0 \\
& a_{R V}=\sin \gamma_{0} \\
& a_{R \gamma}=V_{0} \cos \gamma_{0} \\
& a_{R R}=a_{R p}=a_{R q}=a_{R r}=a_{R \alpha}=a_{R \beta}=a_{R \sigma}=0 \\
& a_{p \beta}=\frac{1}{I_{x x}} \frac{\partial C_{l}}{\partial \beta} \bar{q}_{0} S_{r e f} b_{r e f} \\
& a_{p V}=a_{p \gamma}=a_{p R}=a_{p p}=a_{p q}=a_{p r}=a_{p \alpha}=a_{p \sigma}=0 \\
& a_{q V}=\frac{M_{0}}{I_{y y} V_{0}} \frac{\partial C_{m}}{\partial M} \bar{q}_{0} S_{r e f} c_{r e f} \\
& a_{q \alpha}=\frac{1}{I_{y y}} \frac{\partial C_{m}}{\partial \alpha} \bar{q}_{0} S_{r e f} c_{r e f} \\
& a_{q \gamma}=a_{q R}=a_{q p}=a_{q q}=a_{q r}=a_{q \beta}=a_{q \sigma}=0 \\
& a_{r \beta}=\frac{1}{I_{z z}} \frac{\partial C_{n}}{\partial \beta} \bar{q}_{0} S_{\text {ref }} b_{r e f} \\
& a_{r V}=a_{r \gamma}=a_{r R}=a_{r p}=a_{r q}=a_{r r}=a_{r \alpha}=a_{r \sigma}=0 \\
& a_{\alpha V}=-\frac{g_{0}}{V_{0}^{2}} \cos \gamma_{0} \cos \sigma_{0}-\frac{1}{m V_{0}^{2}}\left(M_{0} \frac{\partial C_{L}}{\partial M}+C_{L}\right) \bar{q}_{0} S_{r e f} \\
& a_{\alpha \gamma}=-\frac{g_{0}}{V_{0}} \sin \gamma_{0} \cos \sigma_{0} \\
& a_{\alpha R}=-\frac{2 g_{0}}{R_{0} V_{0}} \cos \gamma_{0} \cos \sigma_{0} \\
& a_{\alpha q}=1 \\
& a_{\alpha \alpha}=-\frac{1}{m V_{0}} \frac{\partial C_{L}}{\partial \alpha} \bar{q}_{0} S_{r e f} \\
& a_{\alpha \sigma}=-\frac{g_{0}}{V_{0}} \cos \gamma_{0} \sin \sigma_{0} \\
& a_{\alpha p}=a_{\alpha r}=a_{\alpha \beta}=0 \\
& a_{\beta V}=\frac{g_{0}}{V_{0}^{2}} \cos \gamma_{0} \sin \sigma_{0} \\
& a_{\beta \gamma}=\frac{g_{0}}{V_{0}} \sin \gamma_{0} \sin \sigma_{0} \\
& a_{\beta R}=\frac{2 g_{0}}{R_{0} V_{0}} \cos \gamma_{0} \sin \sigma_{0} \\
& a_{\beta p}=\sin \alpha_{0} \\
& a_{\beta r}=-\cos \alpha_{0} \\
& a_{\beta \beta}=-\frac{1}{m V_{0}} \frac{\partial C_{S}}{\partial \beta} \bar{q}_{0} S_{r e f} \\
& a_{\beta \sigma}=-\frac{g_{0}}{V_{0}} \cos \gamma_{0} \cos \sigma_{0} \\
& a_{\beta q}=a_{\beta \alpha}=0 \\
& a_{\sigma V}=\frac{\tan \gamma_{0} \sin \sigma_{0}}{m V_{0}^{2}}\left(M_{0} \frac{\partial C_{L}}{\partial M}+C_{L}\right) \bar{q}_{0} S_{r e f} \\
& a_{\sigma \gamma}=\frac{L_{0}}{m V_{0}} \sin \sigma_{0} \\
& a_{\sigma p}=-\cos \alpha_{0} \\
& a_{\sigma r}=-\sin \alpha_{0} \\
& a_{\sigma \alpha}=\frac{\tan \gamma_{0} \sin \sigma_{0}}{m V_{0}} \frac{\partial C_{L}}{\partial \alpha} \bar{q}_{0} S_{r e f}
\end{aligned}
$$




$$
\begin{gathered}
a_{\sigma \beta}=\frac{\tan \gamma_{0} \cos \sigma_{0}}{m V_{0}} \frac{\partial C_{S}}{\partial \beta} \bar{q}_{0} S_{r e f}-\frac{L_{0}}{m V_{0}}+\frac{g_{0}}{V_{0}} \cos \gamma_{0} \cos \sigma_{0} \\
a_{\sigma \sigma}=\frac{L_{0}}{m V_{0}} \tan \gamma_{0} \cos \sigma_{0} \\
a_{\sigma R}=a_{\sigma q}=0
\end{gathered}
$$

\section{A.2 Closed-Loop Rotational Model}

For control design purposes, the state-space model is reduced to consider the rotational motion only, by setting $\Delta \dot{V}=\Delta \dot{\gamma}=\Delta \dot{R}=\Delta V=\Delta \gamma=\Delta R=0$. Defining the moment fractions, $\eta_{x}, \eta_{y}, \eta_{z}$, as the control variables, the state-space model of the rotational motion of the closed-loop system, is defined as follows:

$$
\left(\begin{array}{c}
\Delta \dot{p} \\
\Delta \dot{q} \\
\Delta \dot{r} \\
\Delta \dot{\alpha} \\
\Delta \dot{\beta} \\
\Delta \dot{\sigma}
\end{array}\right)=\left[\begin{array}{cccccc}
0 & 0 & 0 & 0 & a_{p \beta} & 0 \\
0 & 0 & 0 & a_{q \alpha} & 0 & 0 \\
0 & 0 & 0 & 0 & a_{r \beta} & 0 \\
0 & a_{\alpha q} & 0 & a_{\alpha \alpha} & 0 & a_{\alpha \sigma} \\
a_{\beta p} & 0 & a_{\beta r} & 0 & a_{\beta \beta} & a_{\beta \sigma} \\
a_{\sigma p} & 0 & a_{\sigma r} & a_{\sigma \alpha} & a_{\sigma \beta} & a_{\sigma \sigma}
\end{array}\right]\left(\begin{array}{c}
\Delta p \\
\Delta q \\
\Delta r \\
\Delta \alpha \\
\Delta \beta \\
\Delta \sigma
\end{array}\right)+\left[\begin{array}{ccc}
b_{p \eta_{x}} & 0 & 0 \\
0 & b_{q \eta_{y}} & 0 \\
0 & 0 & b_{r \eta_{z}} \\
0 & 0 & 0 \\
0 & 0 & 0 \\
0 & 0 & 0
\end{array}\right]\left(\begin{array}{c}
\eta_{x} \\
\eta_{y} \\
\eta_{z}
\end{array}\right)
$$

with elements of matrix A, i.e., $a_{i j}$, are the same of those provided in Section A.1, excluding the coefficients related to the translational motion. The elements of matrix $\mathbf{B}$, i.e., $b_{i j}$, can be specified as:

$$
b_{p \eta_{x}}=\frac{\mathscr{L}_{\text {max }}}{I_{x x}}, \quad b_{q \eta_{y}}=\frac{\mathscr{M}_{\text {max }}}{I_{y y}}, \quad b_{r \eta_{z}}=\frac{\mathscr{N}_{\text {max }}}{I_{z z}}
$$

with $\mathscr{L}_{\text {max }}, \mathscr{M}_{\text {max }}, \mathscr{N}_{\text {max }}$ being the maximum available moments around the roll-, pitch-, and yaw-axes, respectively. The moment fractions, $\eta_{x}, \eta_{y}, \eta_{z}$, are defined as the ratio between the commanded moments and the maximum available moments, so they can be mathematically defined as:

$$
\eta_{x}=\frac{\mathscr{L}_{c m d}}{\mathscr{L}_{\text {max }}}, \quad \eta_{y}=\frac{\mathscr{M}_{c m d}}{\mathscr{M}_{\text {max }}}, \quad \eta_{z}=\frac{\mathscr{N}_{c m d}}{\mathscr{N}_{\text {max }}}
$$

which implies that $\eta \in[-1,1]$.

Moreover, a state-space model that describes the rotational motion of HORUS along its re-entry trajectory is needed for the verification of the control system. Along the re-entry trajectory, HORUS can be controlled by means of the elevation, aileron, rudder, and the thruster moments in $x, y, z$ directions, which are now the control variables. This state-space model is defined as follows:

$$
\begin{aligned}
\left(\begin{array}{c}
\Delta \dot{p} \\
\Delta \dot{q} \\
\Delta \dot{r} \\
\Delta \dot{\alpha} \\
\Delta \dot{\beta} \\
\Delta \dot{\sigma}
\end{array}\right] & {\left[\begin{array}{cccccc}
0 & 0 & 0 & 0 & a_{p \beta} & 0 \\
0 & 0 & 0 & a_{q \alpha} & 0 & 0 \\
0 & 0 & 0 & 0 & a_{r \beta} & 0 \\
0 & a_{\alpha q} & 0 & a_{\alpha \alpha} & 0 & a_{\alpha \sigma} \\
a_{\beta p} & 0 & a_{\beta r} & 0 & a_{\beta \beta} & a_{\beta \sigma} \\
a_{\sigma p} & 0 & a_{\sigma r} & a_{\sigma \alpha} & a_{\sigma \beta} & a_{\sigma \sigma}
\end{array}\right]\left(\begin{array}{c}
\Delta p \\
\Delta q \\
\Delta r \\
\Delta \alpha \\
\Delta \beta \\
\Delta \sigma
\end{array}\right)+} \\
& +\left[\begin{array}{cccccc}
0 & b_{p a} & 0 & b_{p x} & 0 & 0 \\
b_{q e} & 0 & 0 & 0 & b_{q y} & 0 \\
0 & b_{r a} & b_{r r} & 0 & 0 & b_{r z} \\
0 & 0 & 0 & 0 & 0 & 0 \\
0 & 0 & 0 & 0 & 0 & 0 \\
0 & 0 & 0 & 0 & 0 & 0
\end{array}\right]\left(\begin{array}{c}
\Delta \delta_{e} \\
\Delta \delta_{a} \\
\Delta \delta_{a} \\
\Delta M_{T, x} \\
\Delta M_{T, y} \\
\Delta M_{T, z}
\end{array}\right)
\end{aligned}
$$

with elements of matrix A, i.e., $a_{i j}$, specified in Section A.1, and the elements of matrix $\mathbf{B}$, i.e., $b_{i j}$, specified as 
follows [Mooij, 2015]:

$$
\begin{gathered}
b_{V e}=b_{V a}=b_{V r}=b_{V x}=b_{V y}=b_{V z}=0 \\
b_{\gamma e}=b_{\gamma a}=b_{\gamma r}=b_{\gamma x}=b_{\gamma y}=b_{\gamma z}=0 \\
b_{R e}=b_{R a}=b_{R r}=b_{R x}=b_{R y}=b_{R z}=0 \\
b_{p a}=\frac{1}{I_{x x}} \frac{\partial C_{l}}{\partial \delta_{a}} \bar{q}_{0} S_{r e f} b_{r e f} \\
b_{p x}=\frac{1}{I_{x x}} \\
b_{p e}=b_{p r}=b_{p y}=b_{p z}=0 \\
b_{q e}=\frac{1}{I_{y y}} \frac{\partial C_{m}}{\partial \delta_{0}} \bar{q}_{0} S_{r e f} c_{r e f} \\
b_{q y}=\frac{1}{I_{y y}} \\
b_{q a}=b_{q r}=b_{q x}=b_{q z}=0 \\
b_{r a}=\frac{1}{I_{z z}} \frac{\partial C_{n}}{\partial \delta_{a}} \bar{q}_{0} S_{r e f} b_{r e f} \\
b_{r r}=\frac{1}{I_{z z}} \frac{\partial C_{n}}{\partial \delta_{r}} \bar{q}_{0} S_{r e f} b_{r e f} \\
b_{r z}=\frac{1}{I_{z z}} \\
b_{r e}=b_{r x}=b_{r y}=0 \\
b_{\beta e}=b_{\beta a}=b_{\beta r}=b_{\beta x}=b_{\beta y}=b_{\beta z}=0 \\
b_{\sigma r}=b_{\sigma r}=b_{\sigma x}=b_{\sigma y}=b_{\sigma z}=0
\end{gathered}
$$




\section{B}

\section{Software Architecture \& Verification}

A computer simulator that allows for simulating the flight of a spaceplane in its environment and computing control commands is an essential tool to examine the flying qualities and controllability characteristics of a hypersonic spaceplane. The development of this kind of simulator generally consists in the implementation of different elements and the integration of these elements in a whole tool. Thus, it represents a very complex task.

Different simulation environments can be used to achieve the objectives of this thesis project. The TU Delft Astrodynamics Toolbox (Tudat) is an open-source simulation environment developed and maintained by staff and students in the research group of Astrodynamics \& Space Missions at the Faculty of Aerospace Engineering (Delft University of Technology). It is constituted by a set of C++ software libraries, that are functional to simulate several astrodynamics applications. However, MATLAB/Simulink® seems a convenient simulation environment for our application. Indeed, it is one of the most used language to design control systems since a control system toolbox is available. Furthermore, the Generic Attitude and Orbit Control Simulator (GAOCS), which has been developed in MATLAB/Simulink $B$ by Dutch Space and documented by Mooij and Ellenbroek [2011] and Mooij and Wijnands [2002], is available to the user.

A crucial point of the software development process is the verification phase that aims at ensuring the software behaves as intended. Using the GAOCS simulator, which presents well verified and documented blocks, facilitates the verification procedure. However, some blocks necessary for our analysis are not part of the available software package, thus they need to be implemented, integrated with the simulator, and carefully tested. Section B.1 gives a description of the software architecture, giving a comprehensive instruction of all its modules. In particular the attitude control system, actuators and the flight dynamics and environment are fully detailed. The verification procedure of all the implemented software modules is illustrated in Section B.2.

\section{B.1 Software Architecture}

The software architecture is broken down into blocks or modules, which perform the required functionalities. The blocks are interconnected by interfaces indicated as signal flows, which help to identify the inputs and the outputs of each blocks. The modularity is an essential feature of a software, since it helps the designer to understand it and re-use it in a clever way with reduced design and verification effort.

Figure B.1 illustrates the top-level architecture of the simulator showing the main modules and the data flows between them. The mission manager block provides the system with the desired or commanded state, which needs to be actuated by the trim \& guidance system module. It should be noticed that the guidance system is not required in this thesis work, as discussed in Chapter 6. Thus, the trim \& guidance system will be indicated in the following as trim-command logic. From this module, the trim and attitude commands are fed into the attitude control system, together with the actual state of the vehicle provided by the navigation system. The control system determines the required moments to realise the command. The commanded moments are translated in actuator commands by the actuator assignment. The actuators are responsible for converting the deflections of the control surfaces, deflection of the thrust vector in case of TVC or a set of thrust moments in case of RCS thrusters into aerodynamic and propulsive forces and moments. The flight dynamics \& environment is the block where the spaceplane's flight is simulated, considering the interaction with the environment where it operates. Here, the effect of the actuator commands on the system are studied and the new actual 


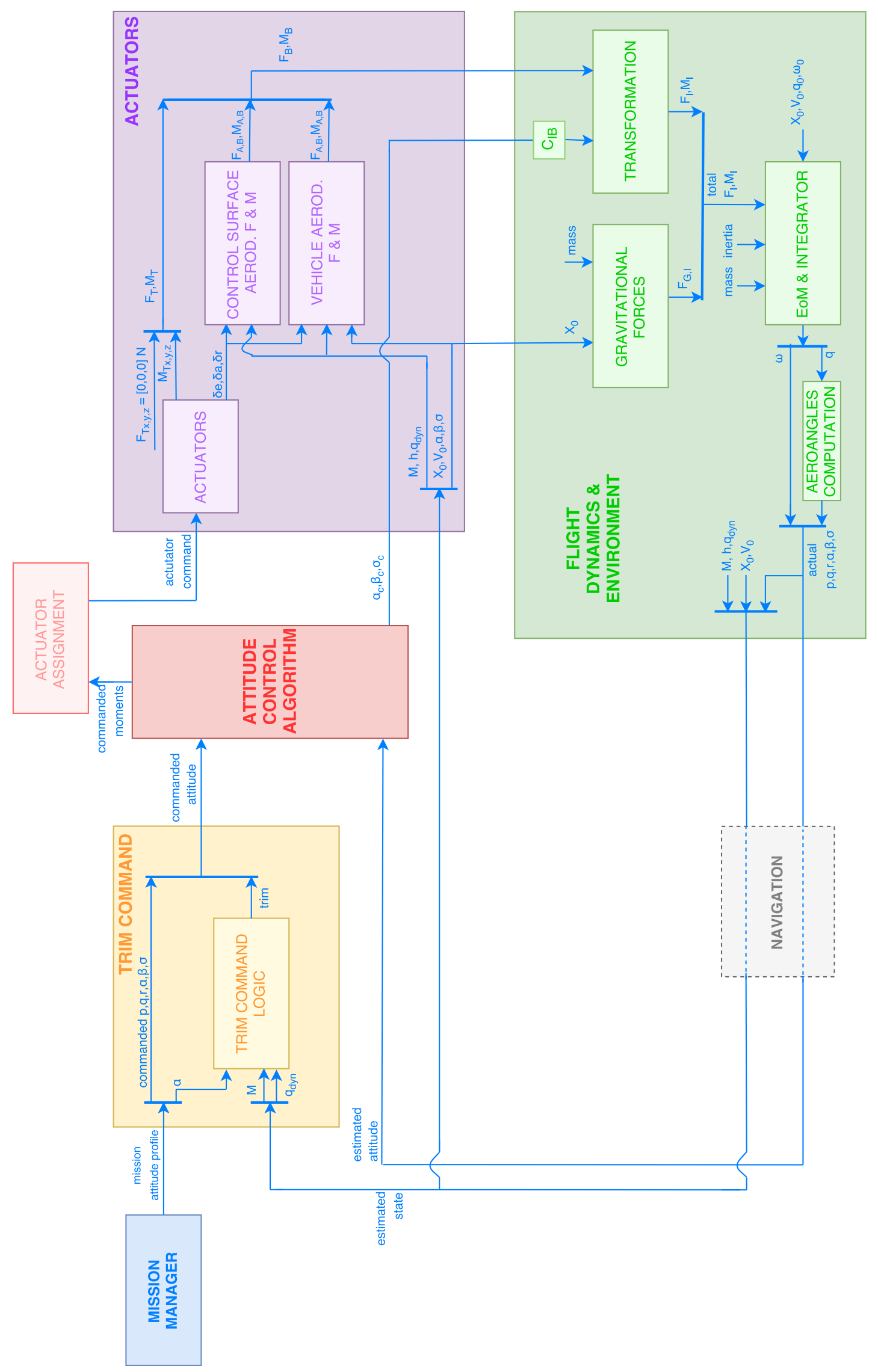

Figure B.1: Software Architecture. 
attitude state is integrated (the fourth-order Runge-Kutta method RK4 with time step $\Delta t=0.005 \mathrm{~s}$ is used). Finally, the navigation system serves, in principle, to determine the actual state of the vehicle on the basis of the measurements provided by the sensors and some internal mathematical law. However, as specified in Chapter 6 , for this analysis the navigation systems can be assumed to perfectly carry out their functions.

All the blocks of the software architecture will be detailed in the following sections, apart from the mission manager and the navigation system blocks. The former will not be further described since it only provides the system with the commanded rotational rate and aerodynamic angles that are defined in the MATLAB workspace by the designer. The latter will not be detailed due to the assumption of an ideal navigation system, which means that the output of the navigation block is again the actual state. In the following the main modules of the simulator will be presented.

\section{Trim-Command Logic}

In Figure B.1 the software architecture of the trim-command logic can be observed, which uses the mission commands and the state of the vehicle to determine the commanded body-flap deflections, elevon deflections and thrust elevation that are necessary to maintain trim stability. This block is part of the GAOCS simulator, thus it does not require additional verification. However, the acceptance test is performed and successfully passed.

\section{Attitude Control System}

The software architecture of the attitude control system is depicted in Figure B.1 as a "black box". However, the software architecture of the LQR and SAC controllers are detailed in Figures 6.4 and 6.7, respectively. The objective of the controller is to calculate the control commands to minimize the deviation between the actual state and the commanded state. The LQR, which is discussed in Section 6.2 serves as benchmark for the more advanced control system, which is the adaptive control system. Since both the control systems are implemented and integrated into the simulator by the author, they need to be verified. The verification procedure is presented in Section B.2.

Once the control commands are computed, the control surfaces deflections, TVC deflections or thrust moments are determined. The actuator assignment module is needed to allocate the commanded moments to the active actuators. This module receives as input the commanded moment fractions and determines the surface deflections, TVC deflections if ascent, and RCS thruster moments if re-entry. Afterwards, the control surface deflections are added to those required for trim stability. The actuator-assignment algorithm is detailed in Chapter 6.4. This block is developed and verified by Brinkman [2017]. Thus, even if it does not require additional verification, an acceptance test is performed.

\section{Actuators}

The software architecture of the actuators module is also illustrated in Figure B.1. Here, the computation of the aerodynamic forces and moments is performed as the summation of the contribution provided by the vehicle itself and the contribution provided by the control-surface deflections. The forces and moments that affect the motion of the vehicle are of aerodynamic and propulsion origin (see Section 4.5). This block is part of the GAOCS simulator, thus additional verification are not required. An acceptance test is performed to verify its functionality.

\section{Flight Dynamics \& Environment}

The Flight Dynamics \& Environment block computes the forces and moments that act on the vehicle in its environment and updates its state. It should be noticed that the forces that are calculated with respect to $\mathscr{F}_{B}$ need to be transform in $\mathscr{F}_{I}$ by means of the transformation matrix $\mathbf{C}_{\mathbf{I}, \mathbf{B}}$ since the EoM are defined with respect to $\mathscr{F}_{I}$. Differently, the moments can be expressed with respect to $\mathscr{F}_{B}$. The software architecture of this block is presented in Figure B.1. In this module the mass properties of the vehicle and the atmospheric properties should be updated. However, since in our application we are considering only a design point at a time, these values remain equal to their nominal values. These properties together with the control forces and moments are inputs to the flight dynamics block, where the EoM integration is performed and the state of the vehicle is updated. This block is part of the GAOCS simulator, thus it does not require additional verification apart from an acceptance test. 


\section{B.2 Verification}

The software verification is crucial for a proper software development. The verification tests aim at verifying that the implemented models reproduces the theoretical models. Thus, these tests are required at each loop of the software development process going from the lowest level to the highest level. Indeed, as shown in the software architecture, the software can be subdivided into blocks that can be implemented independently and are interconnected through interfaces. Each block is represented by a code composed by several functions. Consequently, the verification tests can be applied to the functions of a code (lowest level), to the blocks of the software architecture (intermediate level), and to the whole software (highest level).

The functions of a code can be considered verified if they reproduce correctly the mathematical formulae and if the generated results equal the outcome of analytical calculations. The verification tests of the code functions are known as unit tests. Once they are verified, they can be integrated to form a functional block, whose verification tests are called system tests. These tests depend on whether the algorithm of the blocks has been already implemented and tested by other researchers of the field and the results (reference data) have been published or not. Thus, if the reference data are available and the validity of the source is ascertained, an acceptance test is necessary: the reference inputs are implemented in our tool and the verification test is passed if the results obtained match with the reference outputs. Differently, if the algorithm has never been implemented before, a verification test needs to be planned.

Once all the block are validated, they can be integrated in a whole software and the verification test (known as integration test) of the final software can be performed. In our thesis project, we desire to do an analysis that was never attempted before, thus reference data are not available to verify the whole tool. A sufficient verification is to investigate whether the algorithm meets the expectations and achieves the desired objectives, i.e., it is able to simulate the FSS-1 motion, compute control commands and allow for the analysis of the flying qualities, controllability and stability characteristics of the FSS-1 spaceplane along its hypersonic flight.

It is beyond the scope of this thesis work to present the verification procedure of every component of the software. However, a brief description of the main unit and system tests performed is given for the sake of completeness. The trim command logic is verified by calculating the trim conditions at different points of the FSS-1 trajectory and comparing the results with those by Daimler-Benz Aerospace [1998]. It resulted that the outcome differs of $\pm 10 \%$ from the reference data, which can be due to the different aerodynamic database used. This difference seems acceptable for the level of accuracy required. A similar acceptance test is conducted to verify the actuator assignment module. In this case the work by Brinkman [2017] is used, where the actuator commands are computed starting from given moment fractions and flight conditions along the HORUS re-entry trajectory. It is ascertained that the same output is obtained by providing the same input. To verify the flight dynamics, several tests have been conceived and performed. The translational motion is verified by simulating the HORUS re-entry flight, starting from the same initial conditions and following the same angle-of-attack and bank-angle profiles [Mooij, 1998b]. The results are compared with the reference data, confirming the success of the test. The same test is used also to verify the aerodynamic database, whose verification procedure is detailed in Section 2.1.2. The rotational motion is verified by simulating the motion of a body of mass $1 \mathrm{~kg}$ and inertia equal to a $3 \times 3$ unit matrix with a zero initial angular position and velocity with respect to $\mathscr{F}_{I}$. The simulation is done in case of no moments or different moments applied to the system. The test was considered successful since the body behaves as expected and, moreover, it reaches the final conditions that were checked by applying the basic theory of a rigid body motion.

The verification tests of the major software blocks implemented during this thesis work is provided in the following of this section to assure that they have been properly implemented and integrated. In particular, the linear model and eigenmotion identification procedure is verified in Section B.2.1. In Section B.2.2 the verifications of the LQR, moment-fractions LQR and SAC control system are presented.

\section{B.2.1 Linear System \& Eigenmotion Evaluation}

The implemented the linear model (described in Section 4.7) and of the procedure to study the eigenmotion characteristics (described in Section 5.1) can be checked in one-go, since they are strongy correlated. An acceptance test needs to be performed with the aim of reproducing, given the same state and control variables as input, the same eigenmotion characteristics of the HORUS re-entry vehicle, which was studied by Mooij [1998b].

The eigenmotion and the corresponding characteristic values are identified for several points of the trajectory. In Table B.1, the results of one eigenvalue and relative eigenvector for the time point $t=0 \mathrm{~s}$ are reported. 
Table B.1: HORUS eigenvalues and corresponding characteristic values for time point $t=0 \mathrm{~s}$.

\begin{tabular}{|c|c|c|c|c|}
\hline$\lambda$ & \multicolumn{2}{|c|}{$\begin{array}{c}\text { Results of } \\
\text { implemented software }\end{array}$} & \multicolumn{2}{|c|}{ Reference data } \\
\hline $\begin{array}{l}\operatorname{Re}(\lambda) \\
\operatorname{Im}(\lambda)\end{array}$ & \multicolumn{2}{|c|}{$\begin{array}{l}-0.7302 \cdot 10^{-7} \\
\pm 0.7415 \cdot 10^{-2}\end{array}$} & \multicolumn{2}{|c|}{$\begin{array}{l}-0.7302 \cdot 10^{-7} \\
\pm 0.7415 \cdot 10^{-2}\end{array}$} \\
\hline $\mathrm{P}[\mathrm{s}]$ & \multicolumn{2}{|c|}{847.3} & \multicolumn{2}{|c|}{847.3} \\
\hline $\mathrm{T}_{1 / 2}[\mathrm{~s}]$ & \multicolumn{2}{|c|}{$0.949 \cdot 10^{7}$} & \multicolumn{2}{|c|}{$0.949 \cdot 10^{7}$} \\
\hline$\zeta[-]$ & \multicolumn{2}{|c|}{$0.985 \cdot 10^{-5}$} & \multicolumn{2}{|c|}{$0.985 \cdot 10^{-5}$} \\
\hline$\omega_{n}[\mathrm{rad} / \mathrm{s}]$ & \multicolumn{2}{|c|}{$0.742 \cdot 10^{-2}$} & \multicolumn{2}{|c|}{$0.742 \cdot 10^{-2}$} \\
\hline $\bar{\mu}$ & $\mathrm{z}[-]$ & $\theta\left[^{\circ}\right]$ & $\bar{z}[-]$ & $\theta\left[^{\circ}\right]$ \\
\hline$\Delta V$ & 0.0128 & 52.59 & 0.0129 & 52.59 \\
\hline$\Delta \gamma$ & $0.9634 \cdot 10^{-6}$ & -88.44 & $0.9637 \cdot 10^{-6}$ & -88.44 \\
\hline$\Delta R$ & 1.00 & 180 & 1.00 & 180 \\
\hline$\Delta p$ & $\sim 0$ & 95.20 & $\sim 0$ & 95.68 \\
\hline$\Delta q$ & $0.1678 \cdot 10^{-3}$ & 57.12 & $0.1678 \cdot 10^{-3}$ & 57.12 \\
\hline$\Delta r$ & $\sim 0$ & 69.70 & $\sim 0$ & 69.56 \\
\hline$\Delta \alpha$ & 0.0226 & -32.88 & 0.0226 & -32.88 \\
\hline$\Delta \beta$ & $\sim 0$ & -17.31 & $\sim 0$ & -17.31 \\
\hline$\Delta \sigma$ & $\sim 0$ & 174.52 & $\sim 0$ & 174.52 \\
\hline
\end{tabular}

The level of precision after the comma is chosen to be the same of the one used in the reference data. The maximum percentage error that was registered is $\pm 0.8 \%$, which is a negligible error. It can be concluded that the linear set of EoM and the algorithm for the evaluation of the eigenmotion characteristics are verified.

\section{B.2.2 Control System}

Since no reference data relative to the past application of the SAC controller to the FSS-1 reference vehicle are available, results obtained for HORUS by previous research can be used, making advantage of the fact that FSS- 1 is derived from HORUS. First, the LQR is designed for HORUS, providing the same inputs of the reference data, so that it can be verified by comparing the results with the work of Rijnsdorp [2017]. Once it is verified, it will be used as benchmark to verify the moment-fraction LQR and SAC control system for the FSS-1 spaceplane. These verification processes are described in the following.

\section{LQR Verification}

To verify the control system the work by Rijnsdorp [2017] is used, where a bank reversal is commanded along the HORUS re-entry trajectory. To conduct the acceptance test, it is necessary that the same model is used and the same inputs are provided to the system. As mentioned before, in this case the linearised EoM, from which the state-feedback control laws are derived, are different because of different control variables used. These are aerodynamic surfaces $\Delta \delta_{e}, \Delta \delta_{a}, \Delta \delta_{r}$, and the roll $\Delta M_{T, x}$, pitch $\Delta M_{T, y}$ and yaw $\Delta M_{T, z}$ thrusters. The EoM of the linearised set (Eq. (A.65)), that describe the longitudinal dynamics, are defined as:

$$
\begin{gathered}
\Delta \dot{q}=\frac{1}{I_{y y}}\left(\frac{\partial C_{m}}{\partial \alpha} \Delta \alpha+\frac{\partial C_{m}}{\partial \delta_{e}} \Delta \delta_{e}\right) \bar{q}_{0} S_{r e f} c_{r e f}+\frac{\Delta M_{T, y}}{I_{y y}} \\
\Delta \dot{\alpha}=\Delta q-\frac{1}{m V_{0}} \frac{\partial C_{L}}{\partial \alpha} \bar{q}_{0} S_{r e f} \Delta \alpha
\end{gathered}
$$

The state-feedback laws for longitudinal control are defined as proportional law as:

$$
\Delta \mathbf{u}=\left(\begin{array}{c}
\Delta \delta_{e} \\
\Delta M_{T, y}
\end{array}\right)=-\left[\begin{array}{ll}
K_{e q} & K_{e \alpha} \\
K_{y q} & K_{y \alpha}
\end{array}\right]\left(\begin{array}{c}
\Delta q \\
\Delta \alpha
\end{array}\right)
$$

and the weighting matrices are:

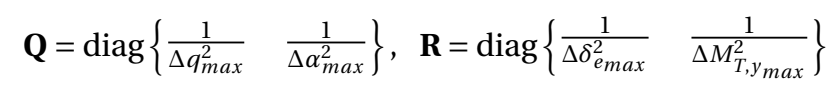

where the control gains can be computed by solving the Riccati equation, as exposed in Section 6.2.1. 
Table B.2: Maximum allowed amplitude of state variables for longitudinal control of HORUS [Rijnsdorp, 2017].

\begin{tabular}{||cccc||}
\hline & Pitch thruster & Pitch thruster and elevators & Elevators \\
$\Delta q_{\max }[\mathrm{deg} / \mathrm{s}]$ & 1.5 & 1.5 & 4 \\
$\Delta \alpha_{\max }[\mathrm{deg}]$ & 1 & 2.5 & 2 \\
\hline
\end{tabular}

Table B.3: Maximum allowed amplitude of state variables for lateral control of HORUS [Rijnsdorp, 2017].

\begin{tabular}{||ccccc||}
\hline & $\begin{array}{c}\text { Roll and } \\
\text { yaw thruster }\end{array}$ & $\begin{array}{c}\text { Roll and } \\
\text { yaw thruster } \\
\text { and ailerons }\end{array}$ & $\begin{array}{c}\text { Roll and } \\
\text { yaw thruster, } \\
\text { ailerons and } \\
\text { rudder }\end{array}$ & $\begin{array}{c}\text { Yaw thruster, } \\
\text { ailerons and } \\
\text { rudder }\end{array}$ \\
$\Delta p_{\max }[\mathrm{deg} / \mathrm{s}]$ & 1.5 & 2.5 & 2.5 & 10 \\
$\Delta r_{\max }[\mathrm{deg} / \mathrm{s}]$ & 1.5 & 1.5 & 10 & 10 \\
$\Delta \beta_{\max }[\mathrm{deg}]$ & 1 & 1 & 4 & 4 \\
$\Delta \sigma_{\max }[\mathrm{deg}]$ & 4 & 4 & 4 & 10 \\
\hline
\end{tabular}

Similarly, the lateral dynamics can be defined as follows:

$$
\begin{gathered}
\Delta \dot{p}=\frac{1}{I_{x x}}\left(\frac{\partial C_{l}}{\partial \beta} \Delta \beta+\frac{\partial C_{l}}{\partial \delta_{a}} \Delta \delta_{a}\right) \bar{q}_{0} S_{r e f} b_{r e f}+\frac{\Delta M_{T, x}}{I_{x x}} \\
\Delta \dot{r}=\frac{1}{I_{z z}}\left(\frac{\partial C_{n}}{\partial \beta} \Delta \beta+\frac{\partial C_{n}}{\partial \delta_{a}} \Delta \delta_{a}+\frac{\partial C_{n}}{\partial \delta_{r}} \Delta \delta_{r}\right) \bar{q}_{0} S_{r e f} b_{r e f}+\frac{\Delta M_{T, z}}{I_{z z}} \\
\Delta \dot{\beta}=\sin \alpha_{0} \Delta p-\cos \alpha_{0} \Delta r-\frac{g}{V_{0}} \cos \gamma_{0} \cos \sigma_{0} \Delta \sigma \\
\Delta \dot{\sigma}=-\cos \alpha_{0} \Delta p-\sin \alpha_{0} \Delta r+\left(\frac{g}{V_{0}} \cos \gamma_{0} \cos \sigma_{0}-\frac{L_{0}}{m V_{0}}\right) \Delta \beta-\frac{\tan \gamma_{0}}{m V_{0}} \cos \sigma_{0} L_{0} \Delta \sigma
\end{gathered}
$$

The control laws for lateral control and weighting matrices can be written as:

$$
\begin{aligned}
& \Delta \mathbf{u}=\left(\begin{array}{c}
\Delta \delta_{a} \\
\Delta \delta_{r} \\
\Delta M_{T, x} \\
\Delta M_{T, z}
\end{array}\right)=-\left[\begin{array}{llll}
K_{a p} & K_{a r} & K_{a \beta} & K_{a \sigma} \\
K_{r p} & K_{r r} & K_{r \beta} & K_{r \sigma} \\
K_{x p} & K_{x r} & K_{x \beta} & K_{x \sigma} \\
K_{z p} & K_{z r} & K_{z \beta} & K_{z \sigma}
\end{array}\right]\left(\begin{array}{c}
\Delta p \\
\Delta r \\
\Delta \beta \\
\Delta \sigma
\end{array}\right)
\end{aligned}
$$

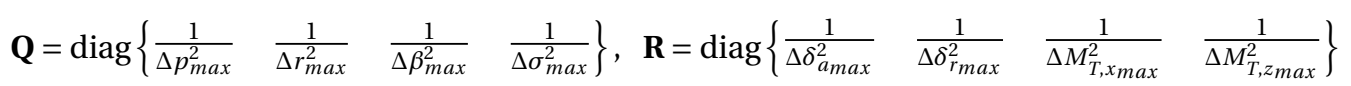

To verify the LQR controller, the inputs to the system are the reference trajectory (which is shown in Figure 2.7) and the maximum allowed amplitude of the state and control variables. The maximum allowed amplitude of the state variables are given in Tables B.2 and B.3 for longitudinal and lateral control, respectively, which vary depending on the active actuators. The maximum allowable amplitudes of control variables are for HORUS [Rijnsdorp, 2017]:

$$
\begin{gathered}
\Delta \delta_{e_{\max }}=40^{\circ} \quad \Delta \delta_{a_{\max }}=40^{\circ} \Delta \delta_{r_{\max }}=40^{\circ} \\
\Delta M_{T, x_{\max }}=1600 \mathrm{Nm} \Delta M_{T, y_{\max }}=10400 \mathrm{Nm} \quad \Delta M_{T, z_{\max }}=7600 \mathrm{Nm}
\end{gathered}
$$

The optimal gains are calculated with these inputs for the HORUS longitudinal and lateral attitude controller. These results coincide with the reference optimal gains. ${ }^{1}$ Once the algorithm to compute the gains is verified, the controller is integrated into the simulator. The verification of the integrated system with the LQR controller is performed through an integration test which consists in simulating the state and control response during a bank reversal manoeuvre along the HORUS re-entry flight and comparing our results with those obtained by Rijnsdorp [2017]. The initial conditions of this bank reversal are shown in Table B.4.

Figure B.2 (left) shows the aerodynamic angle responses for a bank reversal obtained from the implemented and integrated software. These results are the same of those obtained by Rijnsdorp [2017]. In the representation the dashed lines define the commanded response, while the solid lines define the LQR response. It shall be

\footnotetext{
${ }^{1}$ The results are confirmed, through private communication, by Erwin Mooij for the same input reference data.
} 
Table B.4: Initial conditions of the bank reversal along the HORUS reference trajectory [Rijnsdorp, 2017].

\begin{tabular}{||lc||}
\hline Parameter & Value \\
Altitude $[\mathrm{m}]$ & 66209.4 \\
Dynamic pressure $[\mathrm{Pa}]$ & 2297.6 \\
Mach number $[-]$ & 18.78 \\
Flight-path angle $\gamma$ [deg] & 0.64 \\
Angle of attack $\alpha$ [deg] & 40 \\
Bank angle $\sigma$ [deg] & 59.8 \\
\hline
\end{tabular}
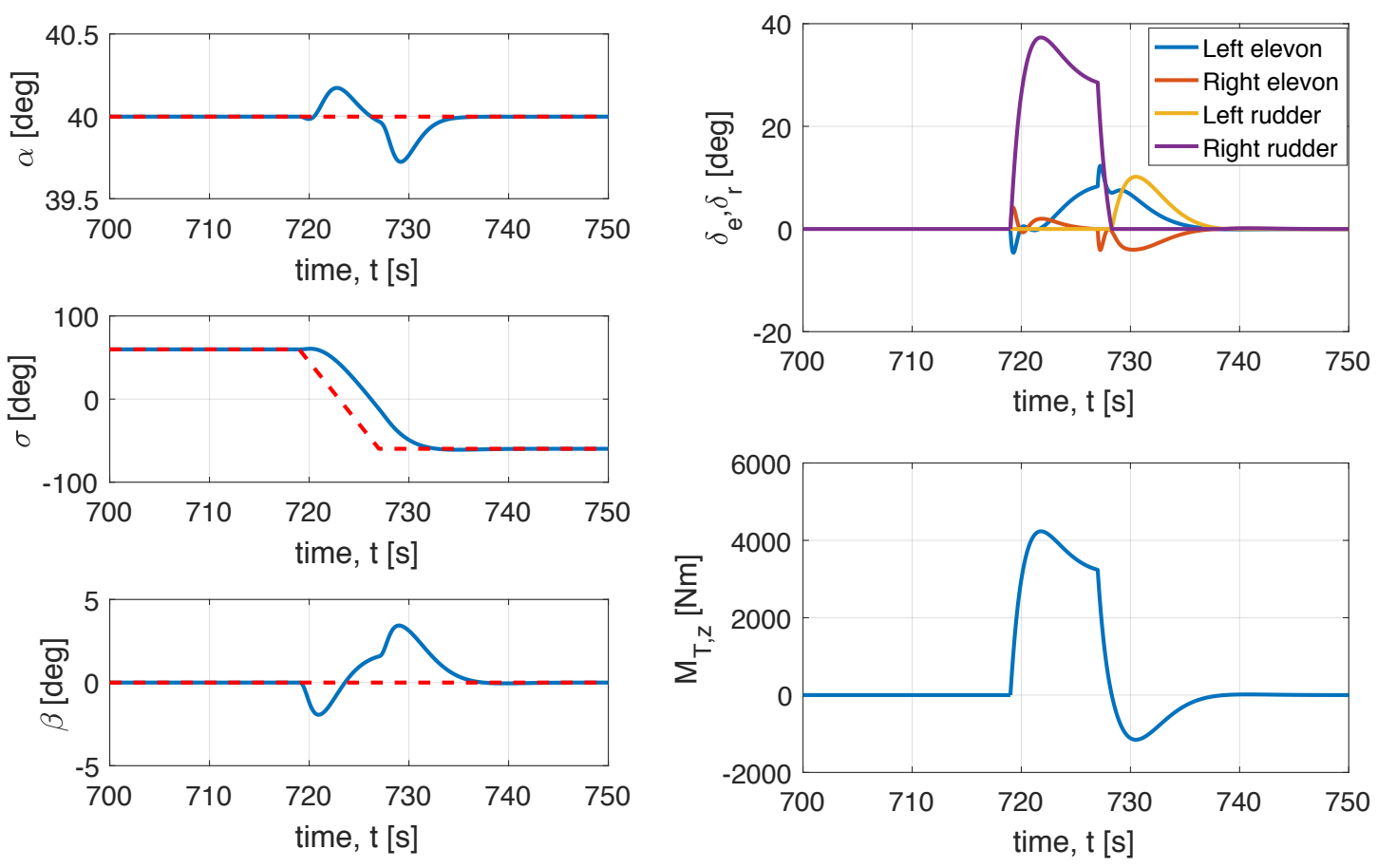

Figure B.2: Aerodynamic angles response for a bank reversal (left) and actuator commands (right).

noticed that the induced aerodynamic-angle variations remain within the allowed boundary specified before. The same is valid for the rotational-rate variations, which return to a value of zero after the bank angle has reached the final commanded value. As an additional note, it could be interesting to also examine the actuator commands, which are presented on the right of Figure B.2. The greatest contribution is given by the right rudder and the yaw thrusters, which is an expected outcome since they are the key actuators to perform the bank-angle commands. It can be concluded that the LQR controller is able to control the vehicle and lets the system reach the commanded conditions within few seconds, thus the simulator integrated with the LQR controller can be considered verified.

At this point it is possible to set up the LQR for the FSS-1 reference vehicle that will be used as benchmark to verify the moment-fraction LQR and the SAC controller. The maximum allowed amplitude of the state and control deviations are the same of those for HORUS, given in Tables B.2 and B.3. However, it is noticed that in the portion of flight where yaw thrusters, elevons and rudder are active, a distinction is needed between dynamic pressure higher and lower than $5000 \mathrm{~Pa}$. It was noted during the analysis that, if for $\bar{q}>5000 \mathrm{~Pa}$ the maximum allowed amplitude of the state variables is equal to the values for $\bar{q}<5000 \mathrm{~Pa}$, the response to a command is quite fast with considerable overshoot and, furthermore, the actuators are required to change very rapidly. The reason behind this is that at higher dynamic pressure the efficiency of the control surfaces is higher, so allowing the same maximum variation of the state variables lead to a nervous behaviour of the control system, which tries to rapidly take the variables back to their allowable values. However, this generates an overall instability on the system. This undesired behaviour can be avoided by allowing greater values of $\Delta p_{\max }=20^{\circ} / \mathrm{s}, \Delta r_{\max }=20^{\circ} / \mathrm{s}$ and $\Delta \sigma_{\max }=20^{\circ}$ for $\bar{q} \geq 5000 \mathrm{~N} / \mathrm{m}^{2}$, so that the actuator oscillations to keep the roll and yaw rates within the bounds are avoided. In this way, the response to input commands is adequate and the operational demands to the actuators is acceptable and feasible. 

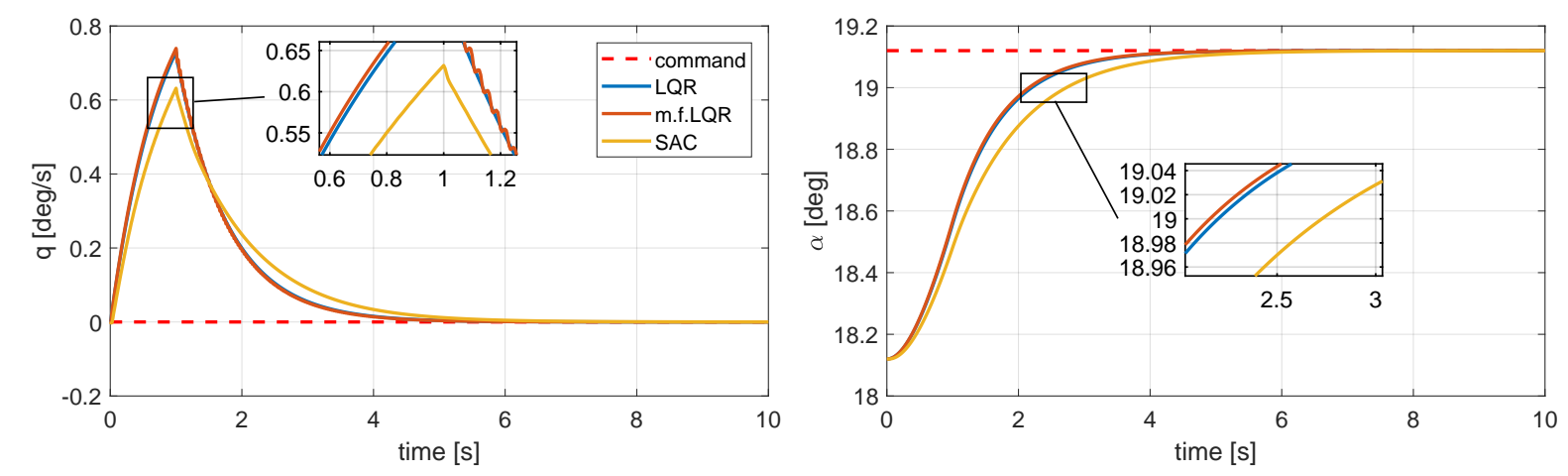

Figure B.3: Verification of the moment-fraction LQR (abbreviated with "m.f.LQR" in the figure) and SAC controller for a control step command in angle of attack. The command is applied to design point with $M=15$ along the re-entry trajectory.

The maximum allowed deflections of the aerodynamic surfaces and maximum thrust momentum of the thruster are defined by the structural limitations of the vehicle in Table 2.6, and reported synthetically here for the sake of completeness [Daimler-Benz Aerospace, 1998]:

$$
\begin{gathered}
\Delta \delta_{e_{\max }}=30^{\circ} \quad \Delta \delta_{a_{\max }}=30^{\circ} \quad \Delta \delta_{r_{\max }}=30^{\circ} \\
\Delta M_{T, x_{\max }}=3500 \mathrm{Nm} \quad \Delta M_{T, y_{\max }}=45700 \mathrm{Nm} \quad \Delta M_{T, y_{\max }}=21400 \mathrm{Nm}
\end{gathered}
$$

\section{SAC Verification}

Once the simulator integrated with the LQR controller has been verified, it can be used as benchmark to validate both the moment-fraction LQR and the SAC controller. On the basis of the theory exposed in Sections 6.2 and 6.3, the response to a $1^{\circ}$-step command in angle of attack is presented for the LQR, moment-fraction LQR and SAC controller in Figure B.3 for initial flight conditions at Mach 15 along the FSS-1 re-entry trajectory. Only $q$ and $\alpha$ are represented since they are the variables that change significantly during the realisation of an angle-of-attack command. As can be seen, the performance of the moment-fraction LQR is very similar to benchmark LQR controller. A zoom-in is shown in the figure to visualise the error between the two LQR, which is of order $\sim 10^{-5}$, thus negligible. The SAC controller shows a similar performance with respect to the other two control systems, being able to properly realise the command. An analogous situation can be described in case of commands in the lateral-directional motion. It can be concluded that the algorithms of both the momentfraction LQR and SAC controller, integrated with the simulator, behave as expected and desired. Thus, the moment-fraction LQR and SAC controller are successfully verified. 